NBS

NBS Standard Reference PUBLICATIONS Materials Catalog 1984-85 n.m (I) A 11107258159
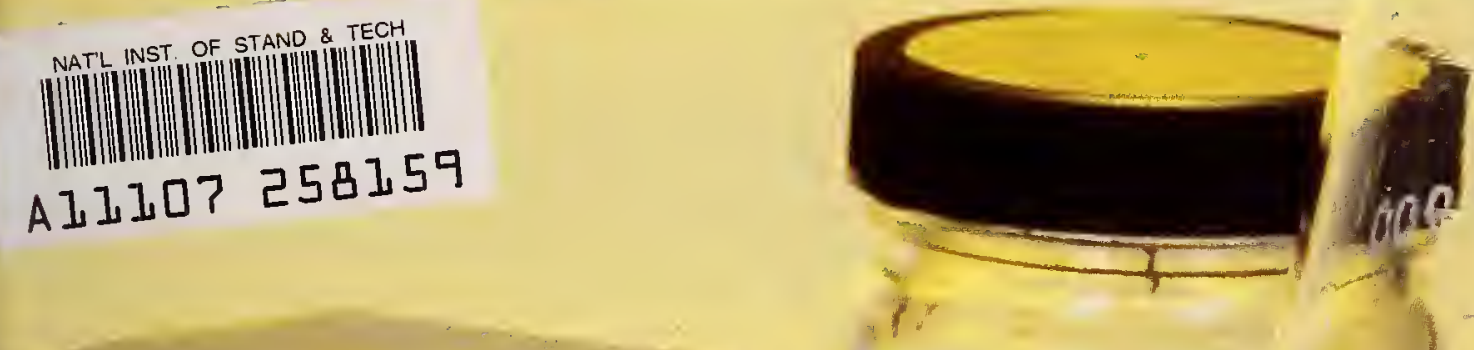

NBS Special Publication 260 


\section{Information and Staff}

\section{Information}

Office of Standard Reference Materials Room B311, Chemistry Building National Bureau of Standards Washington, DC 20234

(Telephone 301-921-2045)

\section{Staff}

Chief: Stanley D. Rasberry

Deputy Chief: William P. Reed

Administrative Officer: Margaret E. Guggenheimer Treva Siedling

Manager, Data Processing: Lee T. Klein

Manager, Production and Certification Programs: Thomas E. Gills

Project Managers:

Robert L. Alvarez

Thomas E. Gills

Lee J. Kieffer

William P. Reed

Richard W. Seward
Promotion and Certification Staff:

Mary H. Blair

Dolly G. Downs

Lisa J. Cooper

Manager, Marketing and Customer Services: Richard W. Seward

Marketing and Sales Staff:

Sandra L. Barber

Patricia A. Brletic

Claudia A. Engel

Julie M. Frum

Catherine H. Hudson

Ruth H. Meyer

Melody V. Smith

Jocelyn A. Washington

Manager, Preparation of Materials and Packaging: Paul A. Lundberg

Preparation and Packaging Staff:

Dale Friend

John F. Savoy

Frances S. Trice

Helen A. Tyler

Manager, Packing and Shipping: Steven G. Webber

Packing and Shipping Staff:

Roger E. Brown

Carleton C. Fisher

James T. Fort 


\section{NBS Special Publication 260}

\section{NBS Standard Reference Materials Catalog 1984-85}

C. H. Hudson, Editor

Office of Standard Reference Materials

National Measurement Laboratory

National Bureau of Standards

Washington, DC 20234

CAUTION: The values given in the following sections are listed primarily as a guide to purchase.

The values shown are nominal and may differ from those shown on the certificates. Space limitations have required that some values be omitted. For these reasons, the certificates issued with the standards should always be consulted to obtain the certified values.

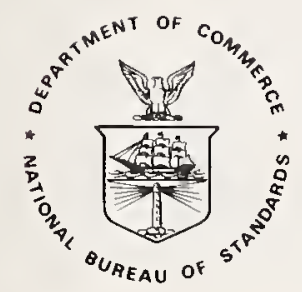

U.S. Department of Commerce Malcolm Baldrige, Secretary

National Bureau of Standards Ernest Ambler, Director 
National Bureau of Standards

Special Publication 260

Supersedes NBS Spec. Publ. 260-1981-83 catalog

Nat. Bur. Stand. (U.S.), Spec. Publ. 260-1984-85

catalog, 180 pages (February 1984)

CODEN: XNBSAV

\section{U.S. GOVERNMENT PRINTING OFFICE WASHINGTON: 1984}

For sale by the Superintendent of Documents,

U.S. Government Printing Office, Washington, DC 20402 


\section{Contents}

1 Abstracts and Key Words

2 Introduction

2 Definitions

3 SRM Catalog

3 Preparation and Availability of Standard Reference Materials

3 Ordering

4 Terms

4 Late Charges

4 Proforma Invoice (Price Quotation)

4 Domestic Shipments

4 Foreign Shipments

5 Documentation

6 Introduction (Spanish)

10 Introduction (French)

14 Introduction (German)

Certified Chemical Composition Standards

Steels (Chip Form)

Plain Carbon Steels

Low Alloy Steels

High Alloy Steels

Stainless Steels

Tool Steels

Steels (Granular Form)

Steels (Solid Form)

Ingot Iron and Low Alloy Steels

Special Ingot Irons and Low-Alloy Steels

Stainless Steels

Group I

Group II

Group III

Specialty Steels

High-Temperature Alloys

Tool Steels

Steelmaking Alloys

Cast Irons (Chip Form)

Cast Steels, White Cast Irons, Ductile

Irons, and Blast

Furnace Irons (Solid Form)

39

39

39

41

42

42

42

43

43

44

44

44

45

45

45

45

46

48

49

49

50

50

51

51

51

52

53

53

53

53

53
Nonferrous Alloys (Chip Form)

Aluminum-Base Alloys

Copper-Base Alloys

Copper "Benchmark"

Lead-Base Alloys

Magnesium-Base Alloys

Nickel-Base Alloys

Trace Elements in Nickel-Base Superalloy

Nickel Oxide

Selenium

Tin-Base Alloys

Titanium-Base Alloys

Zinc-Base Alloys

Zirconium-Base Alloys

Nonferrous Alloys (Solid Form)

Aluminum "Benchmark"

Copper-Base Alloys

Copper "Benchmark"

Lead-Base Alloys

Nickel-Base Alloys

Titanium-Base Alloys

Zinc-Base Alloys

Zirconium-Base Alloys

Gases in Metals

Certified for Hydrogen, Oxygen, and Nitrogen

High-Purity Metals

Microanalytical

Mineral Glasses for Microanalysis

Glasses for Microchemical Analysis

Cartridge Brass

Fe-Cr-Ni Alloy 
Tungsten-20\% Molybdenum

Gold-Silver

Gold-Copper

Iron-3.22\% Silicon

Primary, Working, and Secondary

Chemicals

Microchemical

Clinical Laboratory

Biological

Environmental

Analyzed Gases

Permeation Tubes

Sulfur Dioxide

Nitrogen Dioxide

Benzene

Analyzed Liquids and Solids

Single Element

Multi Element

Organic Constituents

Polychlorinated Biphenyls in Oil

Sulfur in Coal

Industrial Hygiene

Freeze-Dried Urine

Materials on Filter Media

Respirable Quartz

Asbestos

Metallo-Organic Compounds

Wear Metals in Oil

Fertilizers

Ores

Rocks, Minerals, and Refractories

Glasses

Carbides

Cements

Trace Elements

Nuclear Materials

Special Nuclear Materials

Plutonium Assay

Plutonium Isotopic

Uranium Assay

Uranium Isotopic

Radiation Dosimetry

Fission Track Glass

Stable Isotopic

Special Nuclear Containers

\section{Certified Physical Properties Standards}

Ion Activity

$\mathrm{pH}$

$\mathrm{pD}$

Ion-Selective Electrodes

Mechanical and Metrology

Scanning Electron Microscope

Optical Microscope Linewidth-

Measurement

Coating Thickness

Nonmagnetic Coating on Magnetic Substrate

Magnetic Coating on Magnetic Substrate

Magnetic Coating on Non-Magnetic Substrate

Gold Coating on Glass Sealing Alloy Gold Coating on Nickel

Glass

Chemical Resistance (Durability) of Glass

Electrical Properties of Glass

Glass Viscosity

Glass Viscosity Fixpoints

Relative Stress Optical Coefficient

Glass Liquidus Temperature

Elasticity

Microhardness

Density

Molecular Weight

Polymer

Rheology

Heat

Superconductive Thermometric Fixed Point Devices

Freezing Point

\section{Defining Fixed Points-International} Temperature Scale

Secondary Reference Points

Melting Point

Calorimetric

Combustion Calorimetric

Solution Calorimetric

Heat Source Calorimetric

Enthalpy and Heat Capacity

Vapor Pressure

Thermal Conductivity

Thermal Expansion

Thermocouple Materials

Magnetic

Magnetic Susceptibility

Magnetic Moment 
Paramagnetic Resonance

Optical

Spectrophotometric

Filters for Spectrophotometry and Luminescence

Reflectance

Specular Spectral Reflectance

Directional-Hemispherical Reflectance

Refractive Index Optical Rotation

Radioactivity

Alpha-Particle, Beta-Particle, Gamma-ray, and Electron-Capture Solution

Alpha-Particle Point-Source

Radiocarbon Dating and Ground Water Studies

Contemporary Standard for Carbon-14 Dating Laboratories

Low-Level Tritiated-Water

Gaseous Radioactivity

Gamma-ray and X-ray Point-Source

Low-Energy-Photon Point-Source

Radium-226 Solution

Radon Analysis

Gamma-Ray Solution

Environmental Natural Matrix Standards for Traceability Tests

Radiopharmaceutical

Special Nuclear Material Packaging

Catalyst Package for Lubricant Oxidation

Metallurgical

Mössbauer

Abrasive Wear

Pitting or Crevice Corrosion

$\mathrm{X}$-ray Fluorescent Emission Target

$X \cdot r a y$ Diffraction

Gas Transmission

Reference Fuel

Electrical Resistivity and Conductivity

Metals

Silicon

Glass

Residual Resistivity Ratio

Conductivity

Nondestructive Evaluation

11 Engineering Type Standards,

Research Materials, and Special

Reference Materials

Standard Rubbers and RubberCompounding Materials

Reference Magnetic Tapes
Centerline Drawings for Optical Character Recognition-B Characters

Sizing Particle Size

Cement Turbidimetric and Fineness

Color

Light-Sensitive Papers and Plastic Chips

Light-Sensitive Papers

Light-Sensitive Plastic Chips

$X$-ray and Photographic

Surface Flammability

Smoke Density Chamber

Tape Adhesion Testing

Research Materials

Special Reference Materials

Differential Thermal Analysis

Melting Point

Specific Surface Area of Powders

\section{Additional Information}

Other Services of the National Bureau of Standards

Calibration and Related Measurement Services

Washington Services

Boulder Services

Office of Weights and Measures

Proficiency Sample Programs

Structural Engineeering-High Capacity

Testing Machine

Accreditation of Testing Laboratories

National Center for Standards and Certification Information

National Standard Reference Data System

Guide for Requesting Development of Standard Reference Materials

Certified Reference Materials from Other Sources

Other NBS Publications in the 260 Series

\section{Indexes}

Category Index of Standards

Alphabetical Index by Standard

Reference Material Name

Numerical Index to Standard Reference Material Certificates 


\section{Abstracts and Key Words}

\section{National Bureau of Standards Catalog of Standard Reference Materials-1984-85}

This Catalog lists those Standard Reference Materials (SRM's) that are available from the National Bureau of Standards (NBS), and those that are soon to be available. The Catalog describes these materials as to their certified characterization, unit size, and type, as well as providing ordering information. Prices for these materials are listed separately in annual supplements to this Catalog.

Key words: accuracy; analysis; calibration; characterization; composition; materials; measurement; properties; Standard Reference Materials.

\section{National Bureau of Standards [Oficina Nacionall de Pesas y Medidas] Catalogo de Materiales Tipo de Referencia-1984-85}

Este Catálogo enumera los Materiales Tipo de Referencia (SRM) que se pueden obtener de la National Bureau of Standards (NBS), y los que pronto estarán disponibles. El Catálogo describe estos materiales de acuerdo con su caracterización certificada, tamaño de la unidad, y tipo, y tambien provee información sobre pedidos. Los precios de estos materiales aparecen separadamente en suplementos anuales de este Catálogo. Palabras claves: precisión; calibración; caracterización; composición; materiales; medida; propiedades; Materiales Tipo de Referencia.

\section{Bureau National des Normes (National Bureau of Standards) Catalogue des Materiels de Reference Standard 1984 -85}

Ce catalogue donne la liste des Matériels de Référence Standard (Standard Reference Materials_SRM) que l'on peut obtenir du Bureau National de Normes (NBS), et de ceux qui seront bientôt disponibles. Le catalogue donne une description de ces matériels concernant leur caractérisation certifiée, la taille et le type de chaque unité, ainsi que les instructions pour rediger la commande. Une liste de prix de ces matériels est donnée séparément chaque année dans des suppléments au catalogue.

Mots-clés: précision; analyse; calibrage; caractérisation; composition; matériels; mesure; propriétés; Matériels de Référence Standard.

\section{National Bureau of Standards \\ Normenprobenverzeichnis 1984-85}

Dieses Verzeichnis enthaelt sowohl diejenigen Normenproben, die zur Zeit vom National Bureau of Standards (NBS) erhaeltlich sind, als auch solche, die in Baelde verfuegbar sein werden. Das Verzeichnis enthaelt ferner eine Beschreibung der Proben nach Art der geprueften Kennzeichnung, nach Einheitsgroesse und Typus, sowie Angaben ueber den Bestellvorgang. Eine Preisliste erscheint alljaehrlich als Nachtrag zu diesem Verzeichnis.

Stichwörter: Genauigkeit; Analyse; Kalibrierung; Kennzeichnung; Zusammensetzung; Materialien; Messung; Eigenschaften; Normenproben. 


\section{Introduction}

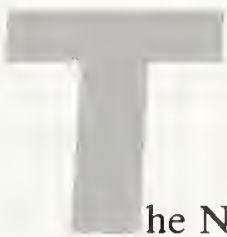

he National Bureau of Standards issues over 1000 different materials through its Standard Reference Materials Program. These materials are primarily Standard Reference Materials (SRM's) certified for their chemical composition, chemical property, or physical property, but also include Research Materials (RM's) and Special Reference Materials (GM's). All SRM's, RM's, and GM's bear distinguishing names and numbers by which they are permanently identified. Thus, each SRM, RM, or GM bearing a given description is identical (within the required or intended limits) to every other sample bearing the same designation-with the exception of individually certified items, which are further identified by serial number.

The first materials issued by NBS were called Standard Samples and consisted of a group of ores, irons, and steels certified for their chemical composition. Since the mid-1960's these materials have been issued as Standard Reference Materials, and cover a wide range of chemical and physical properties and an equally wide range of measurement interests.

\section{Definitions}

The different terms, SRM, RM, or GM, are used to indicate differences in the types of information supplied and in the purposes for which the material is intended. (The terms RM and GM are no longer being used as prefixes for these materials; the numbers 8000 through 9999 are reserved for RM's and GM's.)

Standard Reference Materials have been characterized by the National Bureau of Standards for some chemical or physical property and are issued with a Certificate that gives the results of the characterization. These results are obtained by one of the three established routes of certification, i.e., measurement of the property using: (1) a previously validated reference method, (2) two or more independent, reliable measurement methods, or (3) a network of cooperating laboratories, technically competent and thoroughly knowledgeable with the material being tested. These routes are described in detail in, "The Role of Standard Reference Materials in Measurement Systems," NBS Monograph 148, 54 pages (Jan. 1975). SRM's are defined as being well-characterized and certified materials produced in quantity to improve measurement science. They are prepared and used for three main purposes: (1) to help develop accurate methods of analysis (reference methods); (2) to calibrate measurement systems used to: (a) facilitate the exchange of goods, (b) institute quality control, (c) determine performance characteristics, or (d) measure some property at the limit of the state-of-the-art; (3) to assure the long-term adequacy and integrity of quality control processes. In these ways, SRM's help ensure the compatibility and accuracy of measurements in many facets of national life-from science and technology to trade and commerce.

Research Materials, unlike SRM's, are not certified. Instead of a Certificate, RM's are issued with a "Report of Investigation," the sole authority of which is the NBS staff member who authored the report. An RM is intended primarily to further scientific or technical research on that particular material. The principal consideration in issuing an RM is to provide a homogeneous material so that investigators in different laboratories are assured that they are investigating the same material.

Special Reference Materials differ from both SRM's and RM's in that NBS does not participate in the characterization of these materials. GM's are reference materials produced and certified or guaranteed by 
other government agencies, standards bodies, or other non-profit organizations. When deemed to be in the public interest and when alternate methods of national distribution do not exist, NBS acts as the distributor for such materials. This service is available to all organizations that qualify and have reference materials that would help solve a national measurement problem.

\section{SRM Catallog}

New Catalogs of NBS Standard Reference Materials are published approximately every three years, listing new materials available, materials in preparation, and deleting discontinued materials. To keep the Catalog current between editions, annual supplements are published that list the current prices charged, and any new materials issued.

The materials listed in this Catalog are separated into several major groups: Chemical Composition Standards, Physical Property Standards, Engineering Type Standards, Research Materials, and Special Reference Materials. The first three groups include SRM's only and are subdivided into the categories shown in the Table of Contents.

The numerical values given in the Catalog to describe the materials' properties are nominal values only and are to be used only as guides in selecting the materials. They are not to be used in lieu of the Certificate issued with the materials.

Note: Some SRM's are not issued with Certificates. These exceptions are noted in both the SRM description and the numerical index.

The numerical index provides the SRM, RM, and GM numbers of all materials listed in the Catalog, together with either the date of the current Certificate or a note to explain the absence of a Certificate. an SRM.

An index by category provides a generic listing of the SRM and the primary constituent or element in

An alphabetical index is also provided listing the specific name of the SRM as indicated on the Certificate of Analysis.

\section{Preparation and Avallabillity of Standard Reference Materials}

New and renewal SRM's are being prepared continually. Prospective users are notified directly of the availability of these new items, and the SRM's are described in the next edition of the catalog. To place your name on the mail list maintained by NBS to notify customers of new materials, please contact the Office of Standard Reference Materials at the address listed for placing orders.

In preparing revewal SRM's, the intention is to complete the renewal before the supply of the existing SRM is exhausted. Frequently, this is not possible and the SRM will be out-of-stock for a time. When this occurs, those ordering the material are so notified and, when feasible, possible substitutes are suggested. When the renewal becomes available, customers who have requested either the previous lot or the renewal are promptly notified.

Renewal SRM's will not usually be identical to their predecessors, but will be quite similar especially with regard to the characteristics certified. Generally, the renewal can be used in place of its predecessor. As an example, when the first 0.1 percent carbon Bessemer steel was prepared in 1909, it was called Standard Sample No. 8. During the following years, a number of renewals, $8 \mathrm{a}, 8 \mathrm{~b}$, etc., were prepared. The current SRM 8j, Bessemer Steel (simulated), 0.1\% Carbon, represents the tenth renewal batch of this material. While each of these batches differs somewhat in detailed analysis, all have had the relatively high level of phosphorus, sulfur, and nitrogen, and low alloy metal content characteristic of this type of material.

It is not possible to supply preceding numbers of the renewal series when the stock is exhausted. If little demand exists or an alternate source of supply becomes available for a material, production may be discontinued permanently.

\section{Ordering}

Orders should be addressed to:

Office of Standard Reference Materials

Room B311, Chemistry Building

National Bureau of Standards

Washington, DC 20234

Telephone: (301) 921-2045

Orders should give number of units, catalog number, and name of the material requested. For example: 1 each, 
No. $11 \mathrm{~h}$, Basic-Open-Hearth Steel, 0.2 percent C. The materials described in this Catalog are distributed only in the units listed or in multiples thereof.

Acceptance of an order does not imply acceptance of any provision set forth in the order contrary to the policy, practice, or regulations of the National Bureau of Standards or the U.S. Government.

Orders received for "out-of-stock" materials are cancelled if only out-of-stock items are ordered. On other orders, shipment is made of available materials and out-of-stock items are cancelled. Back-orders are not accepted for out-of-stock materials; if a renewal lot of material is available, it will be furnished automatically.

\section{Terms}

Prices quoted are in U.S. dollars, and are published in the SRM Price List. When SRM Price Lists are issued they are sent to persons or organizations who have requested them. These prices are subject to revision without notice and orders will be billed for the prices in effect at the time of shipment. No discounts are given on purchases of SRM's, RM's, or GM's.

Remittances of the purchase price need not accompany purchase orders. Payment of invoices is expected within 30 days of receipt of an invoice. Payment on foreign orders may be made by any of the following:
a. banker's draft against U.S.A. bank
b. bank to bank transfer to U.S.A. bank
c. cash against documents
d. sight draft
e. International Money Order
f. UNESCO coupons

Letters of Credit cannot be accepted. If a Letter of Credit or any method of payment other then those listed above is to be used, you must secure the services of an agent in the United States to act in your behalf. Your agent would purchase the material and our invoice would indicate that he is the purchaser. The material would be shipped to your agent, who would transship in accordance with your instructions.

NBS cannot "prepay and add" shipping charges to the invoice. Restricted categories such as hydrocarbons, organic sulfur compounds, compressed gasses, rubber compounding materials, radioactive standards, and similar materials are shipped FOB Gaithersburg, MD.

\section{Late Charges}

Unless otherwise notified, payment for SRM's is due within 30 days of shipment of the order to the customer. For non-Federal customers, the U.S. Treasury regulations require late charges, based on the current value of funds to Treasury, be assessed for each 30-day period or portion thereof that the payment is overdue.

\section{Proforma Involice (Price Quotation)}

Proforma invoice service will frequently require three to four weeks to process, and will be furnished only to those requiring such service.

\section{Domestic Shipments}

Shipments of material (except for certain restricted categories) intended for the United States and Canada are normally shipped prepaid (providing that the parcel does not exceed the weight limitations as prescribed by postal laws and regulations).

\section{Forelgn Shipments}

The regulations of various nations covering the importation of SRM's, GM's, and RM's differ widely; any attempt to list all possible variations would be impractical. Therefore, where the shipping practices outlined below do not apply, purchasers will be informed of the best method of shipment for their countries.

Most orders will be shipped by prepaid International Air Parcel Post. Exceptions are items in restricted categories and those shipments that exceed parcel post weight limitations. These exceptions will be shipped FOB Gaithersburg, MD, unless an agent (shipping or brokerage firm) located in the United States is 
required. Where an agent is required, the purchaser will be so notified and will be requested to designate an agent of his/her choice. In this case, the material will be packaged for overseas shipment and will be forwarded to the agent FOB Gaithersburg, MD.

\section{Documentation}

Listed below are the only documents that we will furnish. All documents are printed in English.
a. six commercial invoices
b. two sight drafts
c. two packing slips
d. customs invoices for Canada, New Zealand, Australia, and South Africa
e. Certificate of Origin
f. parcel post receipts for parcel post shipments
g. air waybill for air shipments

If documents other than those listed above are required, the services of an agent in the United States will be needed to purchase and ship the materials.

Note: Orders and inquiries submitted in English will be processed more rapidly than those requiring translations. 


\section{Introductión}

a NBS emite mas de 1,000 materiales diferentes por medio de su Programa de Materiales Tipo de Referencia. Estos materiales son principalmente Materiales Tipo de Referencia (SRM) que han sido certificados por su composición química, propiedades químicas, o propriedades físicas, pero tambien incluyen Materiales de Investigación (RM) y Materiales de Referencia Especial (GM). Todos los SRM, RM y GM tienen nombres y números distintivos por los cuales son identificados permanentemente asi que cada SRM, RM o GM con una descripcion dada es idéntico (dentro de los limites requeridos o proyectados) a cada muestra que tenga la misca designación-con la excepción de artículos individualmente certificados, que además son identificados por un número de serie.

Los primeros materiales emitidos por la NBS se llamaban Muestras Tipo y consistían de un grupo de minerales, hierros y aceros certificados por su composición química. Desde mediados de la década del 1960 se han distribuido estos materiales como Materiales Tipo de Referencia, y cubren una gran extension de propiedades químicas y físicas e igualmente gran extensión de intereses en medición.

\section{Definticiones}

Los diferentes términos, SRM, RM o GM, se usan para indicar diferencias en el tipo de información suministrada y en los propósitos a los cuales el material esta destinado. (Ya no se usan los terminos RM y GM como prefijos para esos materiales; los números desde 8000 hasta 9999 están reservados para RM y GM.)

Los Materiales Tipo de-Referencia han sido caracterizados por la NBS por alguna propiedad química o física y son distribuidos con un Certificado que contiene los resultados de la caracterización. Estos resultados se obtienen mediante uno de los tres métodos de certificación establecidos, es decir, medición de la propiedad por medio de: (1) un método de referencia previamente validado, (2) dos o más métodos independientes y fidedignos de medición, o (3) una red de laboratorios cooperativos que sean técnicamente competente y de completo conocimiento sobre el material que se está probando. Estos métodos se describen en detalle en "The Role of Standard Reference Materials in Measurement Systems" [El Papel de Materiales Tipo de Referencia en Sistemas de Medición], Monografía 148 de la NBS, 54 páginas (enero de 1975). Se definen los SRM como materiales bien caracterizados y certificados que se producen en cantidad para mejorar la ciencia de metrología. Se preparan y usan para tres propósitos principales: (1) para ayudar a desarrollar metodos precisos de análisis (metodos de referencia); (2) para calibrar sistemas de medición que se usan para (a) facilitar el intercambio de mercancías, (b) establecer control de la calidad, (c) determinar características de funcionamiento, o (d) medir alguna propiedad con las técnicas mas avanzadas; (3) para asegurar que los procesos de control de calidad se mantengan adecuados e íntegros por un largo periodo. De esta forma, los SRM ayudan a asegurar la compatibilidad y exactitud de medidas en muchos aspectos de la vida nacionaldesde ciencia y tecnología hasta comercio.

Los Materiales de Investigación (Research Materials), a diferencia de los SRM, no se certifican. En vez de un certificado, los RM se emiten con un "Informe de Investigación," del cual la unica autoridad es el miembro de NBS que escribió el informe. El propósito principal de un RM es fomentar la investigación científica o 
técnica sobre ese material en particular. La principal consideración en la emision de un RM es de proveer un material homogéneo de manera que los investigadores en diferentes laboratorios tengan seguridad de que están investigando el mismo material.

Los Materiales Especiales de Referencia difieren tanto de los SRM como de los RM en que NBS no participa en la caracterización de esos materiales. Los GM son materiales de referencia producidos y certificados o garantizados por otras agencias gubernamentales, organismos de normalización, u otras organizaciones con fines lucrativos. Cuando ello se considera en el interes público y cuando no existen metodos alternativos de distribución nacional, la NBS actúa como distribuidora de tales materiales. Este servicio está a disposicion de todas las organizaciones calificadas que tienen materiales de referencia que ayudarían a resolver un problema nacional de medición.

\section{Catálogo de SRM}

Nuevos catálogos de Materiales de Referencia Regular de la NBS son publicados aproximadamente cada 3 años, enumerando nuevos materiales disponibles, materiales en preparación, y omitiendo materiales descontinuados. Para mantener el catálogo al día entre ediciones, se publican suplementos anuales que dan los precios actúalizados asi como todo material nuevo.

Los materiales enumerados en este catálogo estan divididos en varias categorías: Patrones de Composición Química, Patrones de Propiedad Física, Patrones de Ingeniería, Materiales de Investigación, y Materiales Especiales de Referencia. Los primeros tres grupos incluyen únicamente los SRM y están subdivididos en las categorías indicadas en el Indice.

Los valores numéricos que se dan en el Catálogo para describir las propiedades de los materiales son unicamente valores nominales y deben usarse solamente como guias para seleccionar los materiales. Ellos no se deben emplear en lugar del valor certificado emitido con el material.

Nota: Algunos SRM no se distribuyen con certificados. Estas excepciones se notan tanto en la descripción del SRM como en el índice numérico.

El índice numérico proporciona los números de los SRM, RM y GM para todos los materiales enumerados en el catálogo, conjuntamente con la fecha del certificado actúal o una nota explicando la ausencia de un certificado.

Un índice por categoría proporciona una lista genérica del SRM y el principal constituyente o elemento en un SRM.

Tambien se proporciona un índice alfabético dando el nombre específico del SRM indicado en el Certificado de Análisis.

\section{Preparación y Disponibilidad de Materiales Tipo}

SRM nuevos y renovados se preparan continuamente. A los usuarios potenciales se les notifica directamente de la disponibilidad de estos nuevos artículos, y los SRM se describen en la edición siguiente del catálogo. Para incluir su nombre en la lista que la NBS mantiene para notificar clientes sobre materiales nuevos, por favor escriba a la Oficina de Materiales Tipo de Referencia, a la dirección indicada para pedidos.

Al prepararse SRM renovados, la intención es completar el reemplazo antes de agotarse los SRM existentes. Frecuentemente esto no es posible y el SRM no será disponsible por algún tiempo. Cuando esto sucede, los que piden el material seran notificados y, cuando posible, substitutos serán sugeridos. Cuando la nueva partida esté disponible, los clientes que hayan pedido la partida anterior, o su reemplazo, serán notificados inmediatamente.

Los SRM renovados usualmente no son idénticos a sus antecesores, pero serán muy similares, especialmente en cuanto a las características certificadas. Generalmente la neuva partida puede usarse en lugar de su antecesor. Por ejemplo, cuando el primer acero Bessemer de 0.1 por ciento de carbón se preparó en el 1909, se le llamo Muestra Regular Num. 8. Durante los años subsiguientes se preparó una serie de renovaciones, 8a, 8b, etc. El actúal SRM 8j, Acero Bessemer (Simulado), 0.1 por ciento de carbón, representa la décima partida de renovación de este material. Aunque cada una de estas partidas difiere en algo en el 
análisis detallado, todas han mantenido el nivel relativamente alto de fósforo, azufre y nitrógeno, y bajo contenido de aleación de metal que son caracteristicos de esté tipo de material.

No es posible suministrar números anteriores de una serie de renovaciones cuando la existencia se ha terminado. La produccion puede ser descontinuada permanentemente si existe poca demanda o si se produjo una fuente alternativa de suministro de un material.

\section{Informacion Sobre Pedidos}

Los pedidos se deben enviar a:

Office of Standard Reference Materials

Room B311, Chemistry Building

National Bureau of Standards

Washington, DC 20234

Teléfono: (301) 921-2045

Los pedidos deben indicar cantidad de unidades, número de catálogo, y nombre del material pedido. Por ejemplo: 1, Num. 11h, Basic-Open-Hearth-Steel 0.2 Por Ciento de C. Los materiales descritos en este Catálogo se distribuyen solamente en las unidades enumeradas o en sus múltiples.

La aceptación de un pedido no significa aceptación de disposición alguna incluida en el pedido que sea contraria a las disposiciones, prácticas o reglamentos del National Bureau of Standards o del Gobierno de los Estados Unidos.

Pedidos de materiales que estan agotados se cancelarán si solamente se han pedido artículos que están fuera de existencia. En respuesta a otros pedidos, los materiales disponibles se enviarán y los artículos fuera de existencia se cancelarán. No se aceptan pedidos de materiales agotados; si una emisión nueva del material está disponible, se le proporcionará automáticamente.

\section{T'erminos}

Los precios citados son en dolares de los Estados Unidos, y son los publicados en la Lista de Precios de SRM. Cuando las Listas de Precios de SRM se publican, estas se envían a usuarios que han hecho compra durante los 12 meses ateriores y a personas u organizaciones que las han solicitado. Estos precios estan sujetos a cambios sin notificación previa y los pedidos se facturarán de acuerdo con los precios en efecto al momento del envío. No se hacen descuentos en las compras de los SRM, RM o GM.

No se requiere pago por el precio de compra acompañando el pedido. Se espera el pago de la factura dentro de 30 días del recibo de la factura. El pago por pedidos del exterior se puede hacer en una de las siguientes formas:

a. giro bancario contra un banco de los Estados Unidos.

b. transferencia de banco a banco a un banco de los Estados Unidos.

c. al contado en cambio de documentos.

d. giro a la vista.

e. giro internacional.

f. cupones de la UNESCO.

No se pueden aceptar Cartas de Crédito. Para usar una Carta de Crédito o cualquier metodo de pago que no esté enumerado arriba, debe obtenerse los servicios de un agente en los Estados Unidos para que actue en el nombre del comprador. Su agente compraría el material y nuestra factura indicaría que el es el comprador. El material se enviaría a su agente, quien so lo enviaría a usted de acuerdo con sus instrucciones.

NBS no puede pagar por adelantado cargos de embarque y añadírlos a la factura. Categorías restringidas como hidrocarburos, compuestos de azufre orgánico, gases comprimídos, materiales de mezcla para caucho, patrones radioactivos, y materiales similares se envían franco a bordo (F.O.B.) Gaithersburg, MD.

\section{Cargos Por Demora}

A menos que se estipule lo contrario, el pago por los SRM vence dentro de los 30 días del envío del pedido al cliente. Para clientes no federales, los reglamentos del Departamento del Tesoro de los Estados Unidos 
requieren que cargos por demora, basados en el valor actúal de fondos al Tesoro, se hagan por cada período de 30 días o porción de tal período que el pago esté atrasado.

\section{Factura Pro Forma}

El servicio de factura pro forma frecuentemente requerirá de 3 a 4 semanas para procesarse, y se proporcionará solamente a aquellos que lo soliciten.

\section{Envíos Internos}

El envío de material (excepto ciertas categorías restringidas) destinado para los Estados Unidos y el Canada normalmente se hace con pago anticipado (siempre y cuando el paquete no exceda las limitaciones de peso prescritas por las leyes y reglamentos postales).

\section{Envios all Exterion}

Los reglamentos de diferentes naciones acerca de la importación de SRM, GM y RM varían mucho; y sería impráctico tratar de enumerar todas las variaciones posibles. Por tanto, cuando las prácticas de embarque que aparecen a continuación no apliquen, se informará a los compradores del mejor método de embarque a sus países.

La mayoría de los pedidos se enviará por Servicio Aereo Internacional de Paquetes Postales con pago adelantado. Las excepciones son artículos en categorías restringidas y aquellos embarques que excedan las limitaciones de peso de paquetes postales. Estas exceptiones se enviarán F.O.B. Gaithersburg, MD, a menos que se requiera un agente (compañía naviera o de corretaje) localizado en los Estados Unidos. Cuando se requiere un agente, se notificará al comprador y se le pedira que nombre un agente de us eleccion. En tal caso, el material será embalado para embarque a ultramar y será enviado al agente F.O.B. Gaithersburg, MD.

\section{Documentación}

A continuacion se enumeran los únicos documentos que se proporcionarán. Todos los documentos se publican en inglés.
a. seis facturas comerciales
b. dos giros a la vista
c. dos hojas de embalaje
d. facturas de aduana para el Canadá, Nueva Zelandia, Australia y Africa del Sur
e. Certificado de Origen
f. recibos de paquetes postales, para envíos de paquetes postales
g. hoja de ruta aérea para envíos aéreos

Si se requieren otros documentos además de los enumerados arriba, se necesitarán los servicios de un agente en los Estados Unidos para comprar y enviar los materiales.

Nota: Los pedidos y preguntas que se envien en inglés seran atendidos mas rapidamente que aquellos que requieran traducción. 


\section{Introduction}

e Bureau of National des Normes émet plus de 1000 matériels différents au moyen de son Programme de Matériels de Référence Standard. Ces matériels sont essentiellement des Matériels de Référence Standard (SRM) porteurs d'un certificat garantissant leur composition chimique, leur propriété chimique, ou leur propriété physique. Cependant, ils incluent aussi des Matériels de Recherche (Research Materials-RM), et des Matériels de Référence Spéciaux (Special Reference Materials-GM). A tous les SRM, RM et GM sont assignés des noms et des numéros distinctifs grace auxquels ils sont identifiés de façon permanente. Par conséquent, chaque SRM, RM ou GM porteur d'une description donnée est identique (dans les limites imposées ou voulues) à tout autre échantillon désigné de la même façon—a l'exception des articles certifiés séparément, qui sont en plus identifiés par un numéro de série.

Les premiers matériels que le NBS ait sortis s'appelaient Echantillons Standard (Standard Samples). Il s'agissait d'un groupe de minerais, de fers et d'aciers porteurs d'un certificat garantissant leur composition chimique. Depuis les années 1960, ces matériels sortent sous l'appellation "Matériels de Référence Standard", et couvrent un large éventail de propriétés chimiques et physiques, de même qu'un éventail tout aussi large d'intérêts de mesures.

\section{Définitions}

Les différents termes de SRM, RM et GM, sont utilisés pour indiquer des différences dans le type de renseignements donnés, ainsi que dans l'usage auquel l'article est destiné. (Les termes RM et GM ne sont plus utilisés commme préfixes pour ces articles; les numéros 8000 a 9000 leur sont reservés.)

Les Matériels de Référence Standard ont été caractérisés par le Bureau National de Normes comme ayant une propriété chimique ou physique particulière, et sont émis avec un certificat qui donne les résultats de leur caractérisatión. Ces résultats sont obtenus par l'un des trois moyens conventionnels utilisés pour délivrer un certificat. Il s'agit de mesurer la propriété en utilisant: (1) une methode a reference préalablement validée, (2) au moins deux méthodes de mesure qui soient independantes l'une de l'autre et fiables, ou bien (3) un réseau de laboratories travaillant en coopération, qui soient techniquement compétents et qui connaissent parfaitement le matériel analysé. Ces moyens sont décrits en detail dans "Le Rôle des Matériels de Référence Standard dans les Systèmes de Mesures" (The Role of Standard Reference Materials in Measurement Systems), Monographie 148 du NBS (NBS Monograph 148), 54 pages (édition de janvier 1975). Les SRM sont définis comme des matériels bien caractérisés et certifiés, qui sont produits en nombre pour perfectionner la science des mesures. Ils sont preparés et utilisés à trois fins principales: (1) pour aider au developpement de méthodes exactes d'analyse (méthodes de référence); (2) pour calibrer les systèmes de mesures utilisés pour: (a) faciliter l'échange de marchandises, (b) instituer le contrôle de qualité, (c) determiner les caracteristiques de fonctionnement du matériel, ou bien (d) mesurer une propriété particulière dans la limite des connaissances scientifiques, et (3) pour assurer la congruité et l'exactitude a long terme des procedés de contrôle de qualité. De cette manière, les SRM aident a assurer la compatibilité et la precision des mesures dans bien des aspects de la vie nationale-de la science et la technologie aux échanges et au commerce.

Les Matériels de Recherche, a l'inverse des SRM, ne sont pas certifiés. Les RM sont emis avec, à la place d'un certificat, un "Rapport d'investigation", accordé par la seule autorité du membre du personnel du NBS qui a produit le rapport. Un RM a pour but essential de faire avancer les recherches scientifiques ou 
techniques sur ce matériel particulier. La première des considérations lors de la sortie d'un RM est de fournir un matériel homogène, afin que les chercheurs de divers laboratoires aient l'assurance d'étudier le même matériel.

Les Matériels de Référence Spéciaux diffèrent des SRM et des RM en ce que le NBS ne participe pas a la caractérisatión de ces materiaux. Les GM sont des matériels de reference émis et certifiés, ou bien garantis, par d'autres agences gouvernementales, par des jurys de norme, ou par d'autres organismes a but non lucratif. Quand il est estimé qu'il s'agit d'intérêt public et qu'il n'existe pas d'autres moyens de distributión nationale, c'est au NBS que revient le rôle de distribuer de tels matériels. Ce service est a la dispositión de tous les organismes qui sont qualifiés et qui possèdent des matériels de référence pouvant aider à résoudre un problème national de mesure.

\section{Le catalogue des SRM}

De nouveaux catalogues des Matériels de Référence Standard du NBS sont publiés environ tous les trois ans. Ces catalogues donnent la liste des nouveaux matériels disponibles, des matériels en preparation, et en omettent les matériels discontinués. Pour garder le catalogue à jour entre deux éditions, des suppléments annuels sont publiés, quit donnent la liste des prix en cours et de tous les matériels nouveaux.

Les matériels repertories dans ce catalogue sont classés en plusieurs groupes principaux: les étalons de composition chimique, les étalons de propriété physique, les matériels de recherche, et les matériels de référence spéciaux. Les trois premiers groupes ne comprennent que des SRM et sont subdivisés en catégories indiquées dans la table des matières.

Les valeurs numériques données dans le catalogue pour décrire les propriétés des matériels ne sont là qu'a titre indicatif et ne doivent être utilisées que comme guide pour choisir les matériels. Elles ne doivent pas etre employées à la place du certificat fourni avec le matériel.

Remarque: certains SRM sont emis sans certificat. Ces exceptions sont indiquées à la fois dans la description du SRM et dans l'index numérique.

L'index numérique donne les numéros SRM, RM et GM de tous les matériels répertoriés dans le catalogue, ainsi que la date du certificat actuel, ou bien une note pour expliquer l'absence du certificat.

Un index par catégorie donne une liste générique des SRM et de leur principal composant ou élément.

Un index alphabetique est également fourni. Il donne le nom exact du SRM tel qu'il est indiqué sur son certificat d'analyse. Les RM et GM sont repertories en tant que groupes uniquement.

\section{Préparation et Disponilbilité des Matériels de Référence Standard}

Les nouveaux SRM et ceux qui sont renouvelés sont préparés continuellement. Les éventuels clients sont mis directement au courant de la disponibilité de ces articles nouveaux, et les SRM sont decrits dans l'édition suivante du catalogue. Si vous desirez avoir votre nom sur la liste d'adresses que le NBS garde afin de prévenir les clients de la production de matériels nouveaux, veuillez contacter le Bureau des Matériels de Référence Standard (Office of Standard Reference Materials) à l'adresse indiquée pour les commandes.

Lorsque l'on prépare des SRM renouvelés, c'est avec l'intentión de terminer le renouvellement avant que le stock actuel de SRM no soit épuisé. Très souvent, ceci n'est pas possible et les SRM ne sont alors pas disponibles pour quelque temps. Lorsque c'est le cas, les personnes ayant passé commande de ce matériel sont prevenues de la situation et, quand c'est possible, on leur propose d'éventuels substituts. Lorsque le stock renouvelé est disponible, les clients ayant demandé soit le lot précédent, soit le nouveau, en sont rapidement prévenus.

Les SRM renouvelés ne sont genéralement pas identiques à ceux qu'ils remplacent, mais y sont tout a fait semblables, surtout en ce qui concerne leurs caracteristiques certifiées. En principe, le SRM renouvelé peut être utilisé à la place de son précedent. Par example, quant, en 1909, le premier acier Bessemer à teneur en charbon de $0.1 \%$ a été préparé, on l'a appelé Echantillon Standard No. 8. Les années suivantes, plusieurs matériels de remplacement, 8a, 8b, etc., ont été préparés. L'actual SRM 8j, l'acier (simulé) Bessemer a teneur en charbon de $0.1 \%$, représente le dixième lot de renouvellement de ce matériel. Alors que ces lots different 
quelque peu les uns des autres dans les détails de leur composition, tous possèdent un niveau relativement élevé de phosphore, de soufre et de nitrogène, et un faible niveau de métaux alliés, ce qui est caractéristique de ce genre de matériel.

Il n’est pas possible de fournir des numerós antérieurs a un lot renouvelé lorsque leur stock est epuisé. Si l'article est peu demandé, ou qu'une autre source d'approvisionnement devient disponible pour un matériel donné, la production de celui-ci peut être arretée définitivement.

\section{Les commandes}

Toute commande doit être envoyée à l'adresse suivante:

Office of Standard Reference Materials

Room B311, Chemistry Building

National Bureau of Standards

Washington, DC 20234

Tel. (301) 921-2045

Les commandes doivent preciser le nombre d'unités désirées, le numéro de référence, et le nom du matériel demandé. Par exemple: 1 unité (1 each), No. $11 \mathrm{~h}$, acier basique de four à sole (Basic-Open-Hearth-Steel), 0.2 pour cent de C. (0.2 percent C.). Les matériels décrits dans ce catalogue sont uniquement livres en nombre entier d'unités indiquées.

L'acceptation d'une commande n'implique pas l'acceptation de toute disposition inclue dans celle-ci et contraire aux normes, aux usages ou aux règlements du Bureau National des Normes ou du gouvernement americain.

Les commandes reçues pour les matériels épuisés sont annulées si tous les articles commandés sont des articles épuisés. Pour les autres commandes, les matériels disponibles sont expédiés, et les articles épuisés sont annulés. Les commandes renouvelées ne sont pas acceptées pour les matériels épuisés; si un lot renouvelé du matériel est disponible, il est automatiquement envoyé.

\section{Modes de palement}

Les prix sont donnés en dollars U.S., et sont publiés sur la liste des prix des SRM. Quand les listes des prix sont publiées, elles sont envoyées aux personnes ou organismes qui en ont fait la demande. Ces prix sont sujets à être révisés sans préavis, et les commandes sont facturées selon les tarifs en vigueur au moment de l'envoi. On ne fait pas de remise sur les achats de SRM, de RM et de GM.

Il n'est pas necessaire d'envoyer de paiement avec la commande. Le paiement de la facture doit etre effectué dans les 30 hours après réception de celle-ci. Le paiement des commandes faites hors des Etats-Unis peut etre effectué de l'une des façons suivantes:

a. par chèque bancaire à préveler sur un compte aux Etats-Unis

b. par transfert bancaire sur un compte aux Etats-Unis

c. en liquide sur presentation de documents

d. par effet à vue

e. par mandat international

f. par bons de l'UNESCO.

Les lettres de credit ne sont pas acceptées. Si une lettre de credit, ou tout mode de paiement autre que ceux indiqués ci-dessus doit être utilisé, vous devrez vous assurer les services d'un representant aux Etats-Unis qui agira en votre nom. Votre représentant fera l'achat du matériel, et notre facture le considérera comme acheteur. Le matériel lui sera expédié, et il le fera suivre selon vos instructions.

Le NBS ne peut pas payer d'avance les frais d'expédition et les inclure dans la facture. Certaines categories de matériel soumises à des restrictions, telles que les hydrocarbures, les composés organiques de soufre, les gaz compressés, les matériels caoutchoutes composés, les matériels standard radioactifs, et autres matériels semblables sont expediés F.O.B. a Gaithersburg (état de Maryland).

\section{Penalites pour pallement tardif}

Sauf avis contaire, le paiement des SRM est dû dans les 30 hours suivant l'expédition de la commande au client. Pour les clients non gouvernementaux, les règles de la Trésorerie americaine exigent que des pénalités 
pour paiement tardif, basées sur la valeur courante de la rente sur l'Etat, soient imposées pour chaque periode de 30 jours ou moins après échéance du paiement.

\section{Facture proforma (Detaill de la facture)}

Le service de facture proforma demande généralement de trois a quatre semaines pour être preparé, et n'est fourni qu'à ceux qui en font la demande.

\section{Envols Internes}

Les matériels (sauf certaines catégories soumises à des restrictions) envoyés aux Etats-Unis et au Canada sont normalement expédiés frais de transport compris-à condition que le colis soit dans les limites de poids imposées par les lois et règlements du service postal.

\section{Envols à l"etranger}

Les reglements de divers pays concernant l'importation de SRM, GM et RM varient considerablement; toute tentative de faire la liste de toutes les différences possibles serait mal aisée. Par conséquent, dans tous les cas ou les usages d'expedition decrits ci-dessus ne sont pas applicables, les clients sont informés du meilleur mode d'expedition pour leur pays.

La plupart des commandes sont expédiées, frais de transport compris, par poste internationale des colis par avoin. Les exceptions sont les articles soumis à des restrictions, et les envois hors des limites de poids pour les paquets postaux. Ces exceptions sont envoyées F.O.B. à Gaithersburg (état de Maryland), à moins qu'un représentant (compagnie d'expédition ou de courtage) etabli aux Etats-Unis ne soit obligatoire. Lorsqu'un representant est necessaire, le client en est prevenu et il lui est demandé d'en désigner un de son choix. Dans ce cas, le matériel est empaqueté en prévision d'un transport à l'étranger, et expédié F.O.B. au representant a Gaithersburg (état de Maryland).

\section{Documentation}

Vous trouverez ci-dessous la liste des seuls documents que nous fournissons. Tous ces documents sont en anglais.

a. six factures commerciales

b. deux effets à vue

c. deux fiches detaillant le contenu du paquet

d. des factures douanières pour le Canada, la Nouvelle-Zelande, l'Australie et l'Afrique du Sud

e. un certificat d'origine

f. des reçus de la poste pour les paquets envoyés par la poste

g. un bordereau d'expédition aerienne pour les envois par avion.

Si des documents autres que ceux indiqués sur la liste ci-dessus sont demandés, les services d'un représentant aux Etats-Unis sont nécessaires pour acheter et envoyer les matériels.

N.B. Les commandes et requêtes soumises en anglais recevront reponse plus rapidement que celles nécessitant une traduction. 


\section{Einleitung}

m Rahmen seines Normenprobenprogramms gibt das National Bureau of Standards ueber tausend verschiedene Proben aus. In erster Linie handelt es sich um Standard Reference Materials (SRM), die nach ihrer chemischen Zusammensetzung und ihrer chemischen und physikalischen Eigenschaften geprueft worden sind und zusaetzlich um sogenannte Research Materials (RM) und Special Reference Materials (GM). Alle SRM (Normenproben), RM (Forschungsproben) und GM (Spezialproben) tragen gesonderte Bezeichnungen und Kennummern, wodurch sie permanent identifiziert werden. Das will heissen, dass jede SRM, RM und GM, die eine bestimmte Beschreibung erhalten hat, identisch ist (innerhalb der erforderlichen oder beabsichtigten Grenzen) mit jeder anderen Probe, die auf die gleiche Art gekennzeichnet ist. Die einzige Ausnahme bilden solche Proben, die einzeln geprueft wurden. Diese tragen eine laufende Nummer zur genaueren Kennzeichnung.

Die ersten von NBS herausgegebenen Materialien trugen die Bezeichnung Standard Samples (Standardproben) und bestanden aus einer Reihe von Erzen, Eisen und Stahlen, deren chemische Zusammensetzung bestaetigt worden war. Seit Mitte der sechziger Jahre werden diese Materialien als SRM herausgegeben. Sie umfassen ein breites Spektrum von chemischen und physikalischen Eigenschaften und ein ebenso breites Spektrum von Messungsinteressen.

\section{Defintionem}

Die SRM, RM oder GM werden auf verschiedene Weise benutzt, um Verschiedenheiten in der Art der uebermittelten Information anzuzeigen, wie auch Unterschiede in der geplanten Anwendung des Materials. (Die Bezeichnungen RM und GM werden nicht mehr als Kenncode fuer diese Materialien benutzt; die Nummern 8000 bis 9999 sind ausschliesslich fuer die RM und GM vorgemerkt.)

Die Standard Reference Materials erhalten vom NBS eine Kennzeichnung nach chemischen oder physikalischen Eigenschaften und werden dann mit einem Zertifikat versehen, das das Ergebnis der Analyse angibt. Diese Ergebnisse werden nach einem der drei traditionellen Begutachtungsverfahren gewonnen, d.h. durch die Messung der jeweiligen Eigenschaft auf Grund (1) einer vorher belegten Pruefungsmethode; (2) zweier oder mehrerer unabhaengiger und verlaesslicher Messverfahren, oder (3) einer Gruppe zusammenarbeitender Laboratorien, die technisch kompetent sind und ueber das zu pruefende Material genauestens Bescheid wissen. "The Role of Standard Reference Materials in Measurement Systems" (Die Rolle der SRM in Messystemen), NBS Monograph 148, 54 pages (Jan. 1975) beschreibt diese Verfahren in Einzelnen. Die SRM lassen sich als genau gekennzeichnete und mit Zertifikat versehene Materialien bezeichnen, die in genuegend grossen Mengen hergestellt werden, um zu einer Verbesserung der Messwissenschaft beizutragen. Sie werden aus hauptsaechlich drei Gruenden ausgearbeitet und benutzt: 1. um genaue analytische Methoden (Referenzmethoden) zu entwickeln; 2. um Messysteme zu kalibrieren, die verwendet werden, um (a) den Warenaustausch zu erleichtern; (b) Qualitaetskontrollen einzufurhren; (c) Leistungscharakteristika festzulegen oder (d) eine bestimmte Eigenschaft an der Grenze des Hoechstentwicklungsstandes zu messen und 3. um die langfristige Zulaenglichkeit und Intergritaet von 
Qualitaets-kontrollsystemen zu gewaehrleisten. Auf diese Weise tragen die SRM dazu bei, die Vereinbarkeit und Genauigkeit von Massen auf den verschiedensten Gebieten-von der Wissenschaft und Technik bis zu Handel und Gewerbe-zu sichern.

Anders als die SRM, enthalten die Research Materials kein Zertifikat, sondern statt dessen einen "Report of Investigation" (Untersuchungsbericht), der einzig und allein auf der Autoritaet des NBS-Angestellten basiert, der den Bericht verfasst hat. Ein RM dient vor allem dem Zweck, wissenschaftliche oder technische Forschungsarbeit an diesem bestimmten Material voranzutreiben. Die hauptsaechliche Ueberlegung bei der Publikation eines solchen RM ist, homogenes Material anzubieten, um Wissenschaftlern in verschiedenen Labors die Gewissheit zu geben; dass sie an denselbem Material Forschung treiben.

Special Reference Materials (FM) unterscheiden sich sowohl von den SRM und den RM darin, dass sich das NBS nicht an der Kennzeichnung dieser Proben beteiligt. Bei den GM handelt es sich um Proben, die von anderen Regierungsbehoerden, Eichaemtern oder auch von sonstigen gemeinnuetzigen Institutionen ausgearbeitet und ueberprueft, bezw. garantiert worden ist. In den Faellen, wo es im oeffentlichen Interesse zu liegen scheint oder wo keine anderweitigen landesweiten Verteilungsverfahren verfuegbar sind, betaetigt sich das NBS als Verteiler derartiger Materialien. Dieser Service wird all jenen Organisationen zur Verfuegung gestellt, die den geltenden Bestimmungen entsprechen und ueber Material verfuegen, das zur Loesung eines nationalen Messproblems beitragen mag.

\section{Das SRM-Verzeichnis}

Neue SRM.Verzeichnisse kommen etwa alle drei Jahre heraus. Sie enthalten Listen von neuem, verfuegbaren Material; von Material, das sich in Vorbereitung befindet und Material, das aus dem Katalog gestrichen worden ist. Um das Verzeichnis in der Zwischenzeit auf dem Laufenden zu halten, werden alle Jahre Nachtraege gedruckt, die eine aktuelle Preisliste, sowie etwaige neue Materialien enthalten.

Die Materialien, die in diesem Verzeichnis aufgefuehrt sind, fallen in mehrere Hauptgruppen: chemische Zusammensetzungsnormen; physikalische Eigenschaftsnormen; Fachnormen; Forschungsmaterialien und Special Reference Materials (also besonderes Quellenmaterial). Die drei ersten Gruppen enthalten nur SRM und sind in die Kategorien unterteilt, die im Inhaltsverzeichnis angegeben sind.

Die im Verzeichnis angegebenen Zahlenwerte, die die Eigenschaften der Proben beschreiben, sind als nominelle Werte zu verstehen und sollten daher nur als Richtwerte zur Auswahl der Materialien benutzt werden — und nicht etwa an Stelle eines Zertifikats.

Anmerkung: Manche SRM werden ohne Zertifikat herausgegeben. Ausnahmen dieser Art sind sowohl im Zusammenhang mit der Beschreibung des jeweiligen SRM, als auch in numerischen Inhaltsverzeichnis angegeben.

Dies Inhaltsverzeichnis enthaelt die SRM-, RM- und GM-Kennziffern aller Materialien, die im Gesamtverzeichnis aufgefuehrt sind, wie auch das Datum des derzeit geltenden Zertifikats oder aber eine Erklaerung fuer das Fehlen desselben.

Ein Verzeichnis nach Kategorien bietet eine allgemeine Liste der SRM, sowie den Hauptteil oder das Hauptelement, das im SRM Erwaehnung findet.

In einem alphabetischen Verzeichnis wird schliesslich die genaue Bezeichnung des SRM-so wie sie in dem Zertifikat der Analyse aufscheint—angefuehrt.

\section{Vorbereltung und Verfuegbarkeit von SRM}

Neue SRM, wie auch Neuauflagen sind staendig in Vorbereitung. Etwaige Abnehmer werden direkt von der Verfuegbarkeit solcher neuer Materialien in Kenntnis gesetzt und die SRM werden auch in der naechstfolgenden Ausgabe des Verzeichnisses beschrieben. Falls Sie wuenschen, dass Ihr Name in die NBSKartei aufgenommen wird, damit Sie von neuen Proben erfahren, dann wenden Sie sich bitte an das Office of Standard Reference Materials, dessen Adresse weiter unten angegeben ist.

Bei Neuauflagen von SRM wird versucht, diese fertigzustellen, bevor der Vorrat an bereits existierenden SRM aufgebraucht ist. Oft ist das jedoch nicht moeglich, was bedeutet, das das betreffende SRM zeitweilig nicht lieferbar sein wird. Sollte das der Fall sein, dann wird der Besteller dahingehend benachrichtigt und wenn angebracht, wird Ersatzmaterial angegeben. Wenn die Neuauflage dann verfuegbar ist, wird der Kunde, der entweder die bisherige Probe oder die Neuauflage bestellt hat, umgehend benachrichtigt.

Normalerweise sind die Neuauflagen nicht mit den bisherigen Proben identisch, jedoch einander aehnlich vor allem mit Bezug auf die geprueften Charakteristika. Im allgemeinen kann die Neuauflage an 
Stelle der vorangegangenen Probe benutzt werden. Als beispielsweise im Jahre 1909 zum ersten Mal 0.1 Prozent kohlenstoffhaltiger Bessemer Flusstahl hergestellt wurde, erhielt er die Bezeichnung Normenprobe No. 8. In den darauffolgenden Jahren wurden eine Reihe von Neuauflagen mit der Ziffer 8a, 8b usw. herausgegeben. Die derzeit gueltige Ausgabe SRM 8j (Bessemer Stahl [simuliert] 0.1 Prozent kohlenstoffhaltig) ist die zehnte Neufassung der Probe. Obwohl diese saemtlichen Proben sich in der Einzelanalyse voneinander etwas unterscheiden, besitzen jedoch alle den charakteristischen, relativ hohen Anteil solchen Materials an Phosphor, Schwefel, Stickstoff, sowie einen niedrigen Metallegierungsgehalt.

Vorangegangene Nummern einer Neuauflagenserie sind nicht lieferbar, wenn der Vorrat erschoepft ist. Im Falle geringen Bedarfs oder des Vorhandenseins einer alternativen Lieferquelle, wird die Herstellung eines bestimmten Materials unter Umstaenden voellig eigestellt.

\section{Bestellinformation}

Office of Standard Reference Materials

Room B311, Chemistry Building

National Bureau of Standards

Washington, DC 20234

Telefon: (301) 921-2045

Bestellungen sollten die Anzahl der Einheiten, die Kennummer im Verzeichnis und die namentliche Bezeichnung der Proben enthalten, z.B. one each, No. 11h, Basic-Open-Hearth Steel, 0.2 percent C. Die in diesem Verzeichnis aufgefuehrten Materialien werden ausschliesslich in den angegebenen Einheiten oder in Mehrfachsaetzen derselben geliefert.

Annahme einer Bestellung bedeutet jedoch nicht die gleichzeitige Annahme irgendeiner in der Bestellung angegebenen Bedingung, sofern diese den Gepflogenheiten, der Praxis oder den Richtlinien des NBS oder der amerikanischen Regierung zuwiderlaeuft.

Falls eine Bestellung ausschliesslich aus "nicht lieferbaren" Proben besteht, wird sie gestrichen. Bei anderen Bestellungen wird das lagernde Material geschickt und das nicht lieferbare gestrichen. Nachbestellungen fuer nicht lieferbares Material werden nicht angenommen. Wenn jedoch Neuauflagen verfuegbar sind, werden diese automatisch geliefert.

\section{Bestimmungen}

Preise sind in US Dollar angegeben und scheinen in der SRM Preisliste auf. Nach Erscheinen dieser SRM Preislisten werden diese an Kunden geschickt, die in den vorangegangen 12 Monaten Einkaeufe getaetigt haben wie auch an Einzelpersonen und Organisationen, die darum ersucht haben. Die Preise moegen ohne vorherige Ankuendigung geaendert werden und Bestellungen werden gemaess der am Versandtag geltenden Preise fakturiert. Ermaessigungen beim Kauf von SRM, RM und GM werden nicht gewaehrt.

Der Kaufpreis muss nicht gleichzeitig mit der Bestellung ueberwiesen werden. Zahlung wird innerhalb von 30 Tagen nach Eintreffen der Faktura erwartet. Zahlung von Bestellungen aus dem Ausland kann auf folgende Weise erfolgen:

a. per Bankscheck auf eine amerikanische Bank ausgestellt;

b. durch Ueberweisung eines Bankwechsels an eine amerikanische Bank;

c. durch Barzahlung;

d. per Tratte;

e. durch internationale Postanweisung;

f. mit UNESCO Kupons.

Kreditbriefe werden nicht akzeptiert. Falls jedoch ein Kreditbrief oder ein sonstiger Zahlungsmodus benutzt werden soll, moege sich der Besteller an einen Agenten in den Vereinigten Staaten wenden, der ihn vertritt. Der Agent kauft das Material und scheint auch als der Kaeufer auf unserer Faktura auf. Das Material wird an den Agenten geliefert und dieser schickt es dann an den Besteller gemaess dessen Anweisungen weiter.

Das NBS kann die Faktura nicht mit dem Vermerk "Vorauszahlung und Aufschlag" der Versandkosten versehen. Materialien, die Beschraenkungen unterworfen sind, werden fob Gaithersburg, MD 
verschickt-so etwa Kohlewasserstoffverbindungen, Schwefelverbindungen, Gase in Druckbehaeltern, Kautschukverbundstoffe, radioaktive Normen und aehnlicke Stoffe.

\section{Strafgebuehren fuer Spaetzahler}

Falls nicht anderweitig angegeben, soll die Zahlung fuer SRM-Bestellungen innerhalb von 30 Tagen des Versanddatums erfolgen. Nach den Bestimmungen des amerikanischen Finanzministeriums sind NichtRegierungskunden dazu angehalten, fuer jede 30-Tage Frist oder Teil derselben eine Strafgebuehr auf ueberfaellige Rechnungen auf der Basis des derzeitigen Wertes an das Finanzministerium zu entrichten.

\section{Proforma Falktura [Preisangabe]}

Die Bearbeitung von Proforma-Fakturen nimmt gewoehnlich drei bis vier Wochen in Anspruch und wird nur denjenigen gewaehrt, die darum ersucht haben.

\section{Inlandlieferungen}

Sendungen von Materialien (ausser solchen, die Beschraenkungen unterworfen sind) an Adressen in den Vereinigten Staaten, oder Kanada erfolgen normalerweise mit vorbezahltem Porto-es sei denn, das Paket uebersteige das vom Postgesetz und den Postbestimmungen zugelassene Hoechstgewicht.)

\section{Auslamdslieferungen}

Die Bestimmungen betreffend den Import von SRM, GM und RM sind in den verschiedenen Laendern ausserrordentlich verschieden. Es wäre daher auch nicht zweckdienlich, die vielen verschiedenen Bestimmungen hier aufzufuehren. In den Faellen, wo die weiter unten angefuehrten Versandbestimmungen nicht gelten, wird der Bezieher von dem guenstigsten Versandmodus fuer sein Land unterrichtet werden.

Die meisten Bestellungen werden mit vorbezahltem Porto als internationales Luftpostpaket versendet. Die Ausnahme bilden Proben, die Beschraenkungen unterworfen sind oder das zulaessige Hoechstgewicht ueberschreiten. Derartige Sendungen werden fob Gaithersburg, MD geliefert, falls nicht ein Agent in den Vereinigten Staaten (eine Versandgesellschaft oder ein Makler) eingeschaltet werden muss. In solchen Faellen wird des Bezieher davon in Kenntnis gesetzt und ersucht, einen Agenten seiner Wahl zu benennen. Die Probe wird dann ueberseemaessig verpackt und dem Agenten fob Gaithersburg, MD zugeschickt.

\section{Dokumentennachweis}

Die folgenden Dokumente sind die einzigen, die von uns bereitgestallt werden. Saemtliche Dokumente sind in englischer Sprache abgefasst.
a. 6 Rechnungen
b. 2 Tratten
c. 2 Packzettel
d. Zolldeklarationen fuer Kanada, Neuseeland, Australien und Sued-afrika
e. Ursprungsbestaetigung
f. Empfangsscheine fuer Paketpostversand
g. Frachtschein fuer Luftfrachtversand

Falls Dokumente anderer Art als die hier angefuehrten benoetigt werden, sollte man sich beim Einkauf und Versand des Materials eines Agenten in den Vereinigten Staaten bedienen.

Anmerkung: In englischer Sprache eingereichte Bestellungen und Anfragen werden schneller bearbeitet als solche, die uebersetzt werden muessen. 


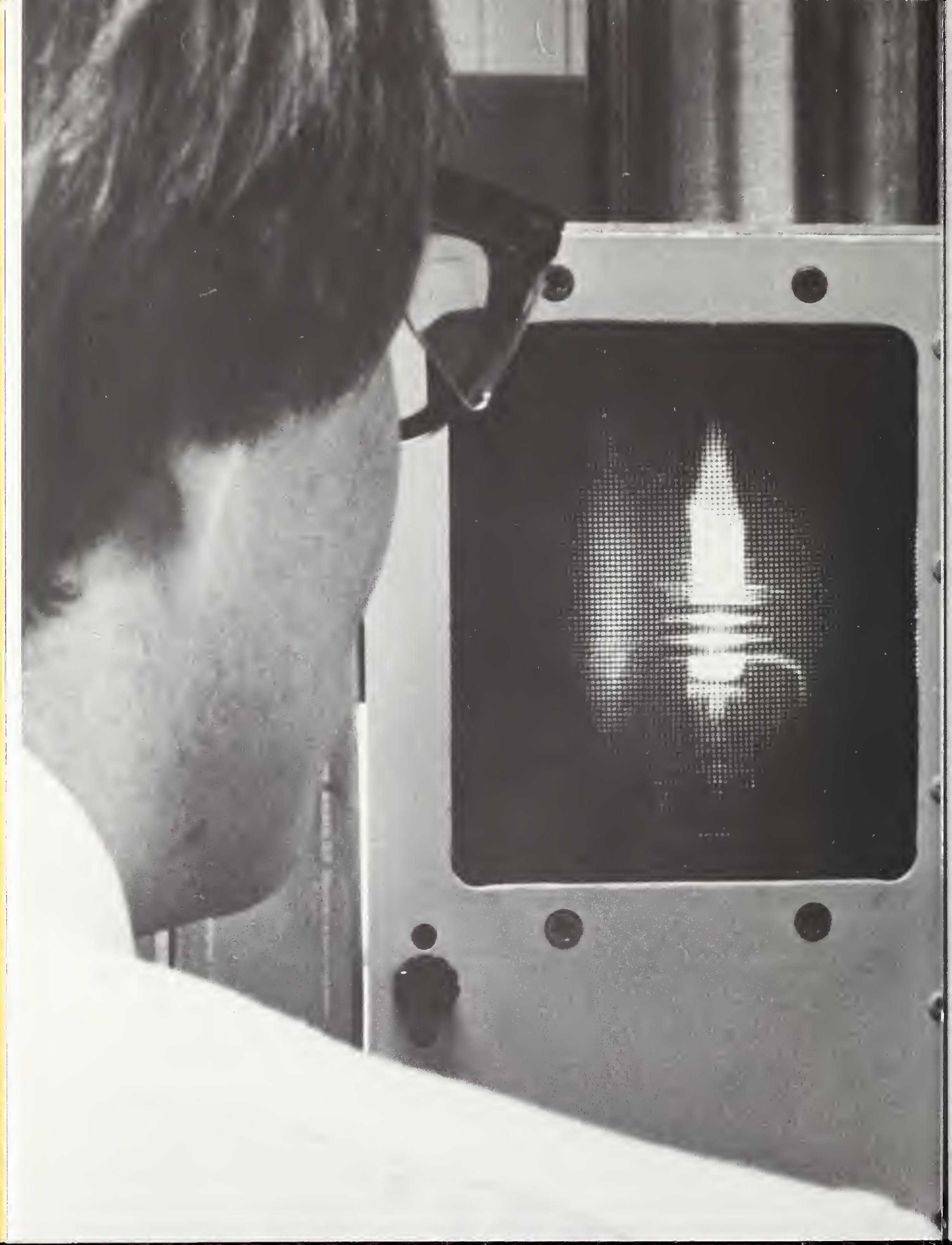




\section{Certified Chemical Composition Standards}

\section{Steels (Chip Form)}

These SRM's were prepared for the steel industry primarily for use with methods involving sample solutions in checking chemical methods of analysis for both production control and customer acceptance. These SRM's consist of nominal composition steel alloys selected to provide a wide range of analytical values for various elements of vital concern to the chemist. They are furnished in 150-gram units (unless otherwise noted) as chips usually sized between 0.4 to $1.2 \mathrm{~mm}$, prepared from selected portions of commercial ingots.

\begin{tabular}{|c|c|c|c|c|c|c|c|}
\hline \multicolumn{8}{|c|}{ Plain Carbon Steels } \\
\hline \multirow{3}{*}{ SRM } & \multirow{3}{*}{ Туре } & \multicolumn{6}{|c|}{ Chemical Composition (Nominal Weight Percent) } \\
\hline & & \multirow[t]{2}{*}{$\mathrm{C}$} & \multirow[t]{2}{*}{ Mn } & \multirow[t]{2}{*}{$\mathbf{P}$} & \multicolumn{2}{|c|}{$\mathbf{S}$} & \multirow[t]{2}{*}{ Si } \\
\hline & & & & & Grav & Comb & \\
\hline $8 \mathrm{j}$ & Bessemer (simulated), $0.1 \mathrm{C}$ & 0.081 & 0.505 & 0.095 & & 0.077 & 0.058 \\
\hline $11 \mathrm{~h}$ & $\mathrm{BOH}, 0.2 \mathrm{C}$ & 0.200 & 0.510 & 0.010 & & 0.026 & $0.21_{1}$ \\
\hline $12 \mathrm{~h}$ & $\mathrm{BOH}, 0.4 \mathrm{C}$ & 0.407 & 0.842 & 0.018 & & 0.027 & 0.235 \\
\hline$\overline{13 g}$ & $\mathrm{BOH}, 0.6 \mathrm{C}$ & 0.613 & 0.853 & 0.006 & & 0.031 & $0.35_{5}$ \\
\hline $14 \mathrm{f}$ & $\mathrm{BOH}, 0.8 \mathrm{C}$ & 0.753 & 0.410 & 0.009 & & 0.039 & 0.172 \\
\hline $15 \mathrm{~g}$ & $\mathrm{BOH}, 0.1 \mathrm{C}$ & 0.094 & 0.485 & 0.005 & & 0.026 & 0.095 \\
\hline $16 f$ & $\mathrm{BOH}, 1.1 \mathrm{C}$ & 0.97 & 0.404 & 0.014 & & 0.026 & 0.214 \\
\hline $19 \mathrm{~g}$ & $\mathrm{AOH}, 0.2 \mathrm{C}$ & 0.223 & 0.554 & 0.046 & 0.032 & 0.033 & 0.186 \\
\hline $20 \mathrm{~g}$ & AISI 1045 & 0.462 & 0.665 & 0.012 & & 0.028 & 0.305 \\
\hline $152 \mathrm{a}$ & $\mathrm{BOH}, 0.5 \mathrm{C}$ (Tin bearing) & 0.486 & 0.717 & 0.012 & & 0.030 & 0.202 \\
\hline 178 & Basic Oxygen 0.4C & 0.395 & 0.824 & 0.012 & & 0.014 & 0.163 \\
\hline 335 & $\mathrm{BOH}, 0.1 \mathrm{C}$ (Carbon only) $300 \mathrm{~g}$ & 0.092 & & & & & \\
\hline 337 & BOH, $1.1 \mathrm{C}$ (Carbon only) $300 \mathrm{~g}$ & 1.07 & & & & & \\
\hline 368 & AISI 1211 & 0.089 & 0.82 & 0.084 & & 0.132 & 0.007 \\
\hline
\end{tabular}




\section{Plain Carbon Steels (Continued)}

\begin{tabular}{|c|c|c|c|c|c|c|c|c|c|c|c|}
\hline SRM & $\mathrm{Cu}$ & $\mathrm{Ni}$ & $\mathrm{Cr}$ & V & Mo & Co & $\mathrm{Ti}$ & Sn & $\underset{\text { (total) }}{\mathrm{Al}}$ & $\mathbf{N}$ & Other \\
\hline $8 \mathrm{j}$ & 0.020 & 0.113 & 0.047 & 0.015 & 0.038 & & & & & & \\
\hline $11 \mathrm{~h}$ & 0.061 & 0.028 & 0.025 & 0.001 & & & 0.004 & & & & \\
\hline $12 \mathrm{~h}$ & 0.073 & 0.032 & 0.074 & 0.003 & 0.006 & & & & $(0.038)$ & 0.006 & \\
\hline $13 \mathrm{~g}$ & 0.066 & 0.061 & 0.050 & 0.001 & & & & & $0.04_{8}$ & & \\
\hline $14 \mathrm{f}$ & 0.072 & 0.053 & 0.070 & 0.002 & 0.013 & & & & 0.060 & & \\
\hline$\overline{15 \mathrm{~g}}$ & 0.036 & 0.017 & 0.028 & 0.001 & & & & & & & \\
\hline $16 f$ & 0.006 & 0.008 & 0.020 & 0.002 & 0.003 & 0.003 & & & & & \\
\hline $19 \mathrm{~g}$ & 0.093 & 0.066 & 0.374 & 0.012 & 0.013 & 0.012 & 0.027 & 0.008 & 0.031 & & $\mathrm{Nb} 0.026$ \\
\hline $20 \mathrm{~g}$ & 0.034 & 0.034 & 0.036 & 0.002 & 0.008 & & & & 0.040 & & \\
\hline $152 \mathrm{a}$ & 0.023 & 0.056 & 0.046 & 0.001 & 0.036 & & & 0.032 & & & \\
\hline 178 & 0.032 & 0.010 & 0.016 & 0.001 & 0.003 & & & & & & \\
\hline 335 & & & & & & & & & & & \\
\hline 337 & & & & & 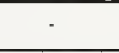 & & & & & & \\
\hline$\overline{368}$ & 0.010 & 0.008 & 0.030 & 0.001 & 0.003 & & & & & 0.010 & \\
\hline
\end{tabular}

Values in parentheses are not certified, but are given for information only.

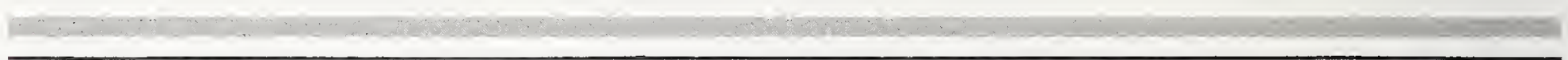

\section{Low Alloy Steels}

\begin{tabular}{|c|c|c|c|c|c|c|c|c|c|c|c|}
\hline \multirow[b]{2}{*}{ SRM } & \multirow[b]{2}{*}{ Type } & \multirow[b]{2}{*}{ (Other Forms) } & \multicolumn{9}{|c|}{ Chemical Composition (Nominal Weight Percent) } \\
\hline & & & $\mathrm{C}$ & Mn & $\mathbf{P}$ & & S & Si & $\mathrm{Cu}$ & $\mathrm{Ni}$ & $\mathbf{C r}$ \\
\hline & & & \multicolumn{9}{|c|}{ Grav Comb } \\
\hline $30 \mathrm{f}$ & $\mathrm{Cr}-\mathrm{V}(\mathrm{SAE} 6150)$ & & 0.490 & 0.79 & 0.011 & & 0.009 & 0.283 & 0.074 & 0.070 & 0.945 \\
\hline $32 \mathrm{e}$ & Ni.Cr (SAE 3140) & & 0.409 & 0.798 & 0.008 & 0.022 & 0.021 & 0.278 & 0.127 & 1.19 & 0.678 \\
\hline $33 \mathrm{~d}$ & Ni-Mo (SAE 4820) & & 0.173 & 0.537 & 0.006 & 0.010 & 0.011 & 0.253 & 0.123 & 3.58 & 0.143 \\
\hline $36 \mathrm{~b}$ & $\mathrm{Cr} 2-\mathrm{Mol}$ & & 0.114 & 0.404 & 0.007 & & 0.019 & 0.258 & 0.179 & 0.203 & 2.18 \\
\hline$\underline{72 \mathrm{~g}}$ & Cr-Mo (SAE X4130) & & 0.278 & 0.492 & 0.009 & & 0.014 & 0.223 & 0.011 & 0.016 & 0.905 \\
\hline $100 \mathrm{~b}$ & Manganese (SAE T1340) & & 0.397 & 1.89 & 0.023 & 0.029 & 0.028 & 0.210 & 0.064 & 0.030 & 0.063 \\
\hline 105 & High-Sulfur 0.2C (Carbon only) & & 0.193 & & & & $(0.60)$ & & & & \\
\hline $106 \mathrm{~b}$ & Cr-Mo-Al (Nitralloy G) & & 0.326 & 0.506 & 0.008 & 0.016 & 0.017 & 0.274 & 0.117 & 0.217 & 1.18 \\
\hline $125 \mathrm{~b}$ & High-Silicon & 1134 & 0.028 & 0.278 & 0.029 & & 0.008 & 2.89 & 0.071 & 0.038 & 0.019 \\
\hline $129 \mathrm{c}$ & High-Sulfur & & 0.125 & 0.769 & 0.076 & & 0.245 & 0.020 & 0.013 & 0.251 & 0.014 \\
\hline $131 \mathrm{c}$ & Low Carbon-Silicon $(100 \mathrm{~g})$ & & 0.0029 & & & & 0.020 & & & & 0.488 \\
\hline $139 \mathrm{~b}$ & Cr-Ni-Mo (AISI 8640) & 1222 & 0.403 & 0.778 & 0.013 & & 0.019 & 0.242 & 0.097 & 0.510 & 0.485 \\
\hline 155 & Cr0.5-W0.5 & & 0.905 & 1.24 & 0.015 & 0.010 & 0.011 & 0.322 & 0.083 & 0.100 & 0.022 \\
\hline 179 & High-Silicon & 1135 & 0.027 & 0.094 & 0.006 & & 0.026 & 3.19 & 0.056 & 0.050 & 1.33 \\
\hline 291 & Cr-Mo (ASTM A213) & & 0.177 & $0.55_{0}$ & 0.008 & & 0.020 & $0.23_{0}$ & 0.047 & 0.065 & $\overline{0.51_{0}}$ \\
\hline 293 & Cr-Ni-Mo (AISI 8620) & & 0.222 & $0.96_{0}$ & 0.018 & & 0.022 & $0.30_{0}$ & 0.032 & $0.48_{0}$ & \\
\hline 361 & AISI 4340 & $661,1095,1261 \mathrm{a}$ & 0.383 & 0.66 & 0.014 & & 0.014 & 0.222 & 0.042 & 2.00 & $0.69_{4}$ \\
\hline 362 & AISI 94B17 (Mod) & $662,1096,1262 a$ & 0.160 & 1.04 & 0.041 & & 0.036 & 0.39 & 0.50 & 0.59 & 0.30 \\
\hline 363 & $\mathrm{Cr}-\mathrm{V}$ (Mod) & $663,1097,1263 \mathrm{a}$ & 0.62 & 1.50 & $0.02_{q}$ & & 0.0068 & 0.74 & 0.10 & 0.30 & 1.31 \\
\hline 364 & High Carbon (Mod) & $664,1098,1264 a$ & 0.87 & $0.25_{5}$ & 0.01 & & 0.0250 & $0.06_{5}$ & $0.24_{9}$ & $0.14_{4}$ & $0.06_{3}$ \\
\hline 1036 & Low Carbon Silicon (25g) & Carbon and Sulf & & & & & 0.0007 & & & & \\
\hline
\end{tabular}




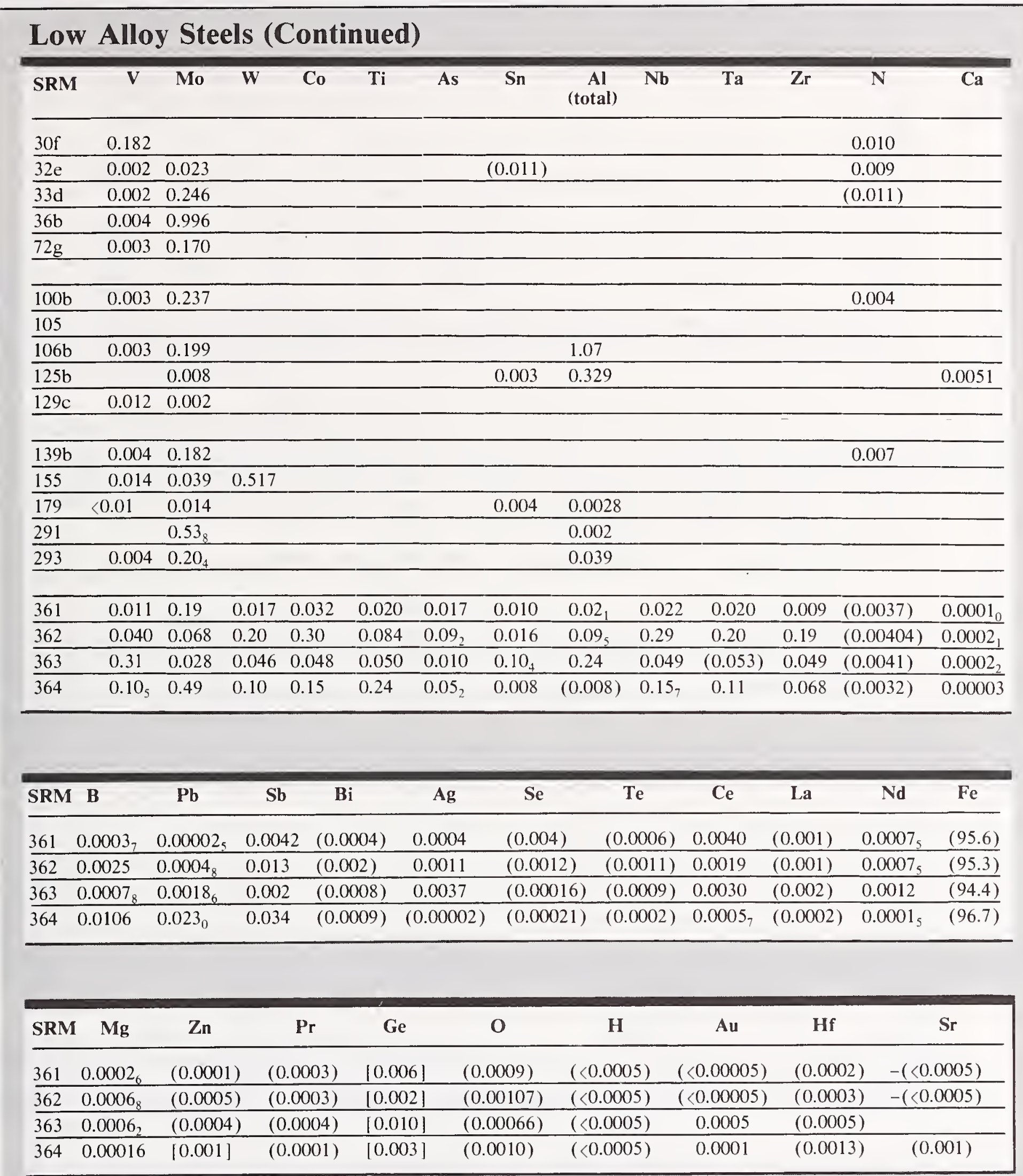

Values in parentheses are not certified, but are given for information only.

Brackets indicate approximate value from heat analysis.

Dash indicates "not detected." Value in parentheses following dash is the conservative "upper limit" of detection. 


\section{High Alloy Steels}

\begin{tabular}{|c|c|c|c|c|c|c|c|c|c|c|c|}
\hline \multirow{3}{*}{ SRM } & & & & & \multicolumn{7}{|c|}{ Chemical Composition (Nominal Weight Percent) } \\
\hline & \multicolumn{3}{|c|}{ Type } & & $\mathbf{C}$ & $\mathbf{M n}$ & $\mathbf{P}$ & \multicolumn{2}{|c|}{$\mathrm{S}$} & \multirow[t]{2}{*}{$\mathrm{Si}$} & \multirow[t]{2}{*}{$\mathrm{Cu}$} \\
\hline & & & & & & & & Grav & Comb & & \\
\hline $126 \mathrm{c}$ & \multicolumn{4}{|c|}{ High-Nickel ( $36 \% \mathrm{Ni})$} & 0.025 & 0.468 & 0.004 & & 0.005 & 0.194 & 0.040 \\
\hline 344 & \multicolumn{4}{|c|}{ Cr15-Ni7-Mo2-All } & 0.69 & 0.57 & 0.018 & & 0.019 & 0.395 & 0.106 \\
\hline 345 & \multicolumn{4}{|c|}{ Cr16-Ni4-Cu3 } & 0.048 & 0.224 & 0.018 & 0.012 & 0.012 & 0.610 & 3.44 \\
\hline 348 & \multicolumn{4}{|c|}{ Ni26-Cr15 (A286) } & 0.044 & 1.48 & 0.015 & & 0.002 & 0.54 & 0.22 \\
\hline SRM & $\mathrm{Ni}$ & $\mathrm{Cr}$ & V & Mo & Co & $\mathrm{Ti}$ & $\underset{\text { (total) }}{\mathrm{Al}}$ & $\mathrm{Nb}$ & $\mathrm{Ta}$ & B & $\mathrm{Fe}$ \\
\hline $126 c$ & 36.05 & 0.062 & 0.001 & 0.011 & 0.008 & & & & & & \\
\hline 344 & 7.28 & 14.95 & 0.040 & 2.40 & & 0.076 & 1.16 & & & & \\
\hline 345 & 4.24 & 16.04 & 0.041 & 0.122 & 0.089 & & & 0.231 & 0.002 & & \\
\hline 348 & 25.8 & 14.54 & 0.25 & 1.3 & & 2.24 & 0.23 & & & 0.0031 & 53.3 \\
\hline
\end{tabular}

\begin{tabular}{|c|c|c|c|c|c|c|c|c|c|}
\hline \multirow{3}{*}{ SRM } & \multirow{3}{*}{ Type } & \multirow{3}{*}{ (Other Forms) } & \multicolumn{7}{|c|}{ Chemical Composition (Nominal Weight Percent) } \\
\hline & & & \multirow[t]{2}{*}{$\mathrm{C}$} & \multirow[t]{2}{*}{ Mn } & \multirow[t]{2}{*}{$\mathbf{P}$} & \multicolumn{2}{|l|}{$\mathrm{S}$} & \multirow[t]{2}{*}{ Si } & \multirow[t]{2}{*}{$\mathrm{Cu}$} \\
\hline & & & & & & Grav & Comb & & \\
\hline $73 c$ & Cr13 (SAE 420) & & 0.310 & 0.330 & 0.018 & & 0.036 & 0.181 & 0.080 \\
\hline $121 \mathrm{~d}$ & Cr17-Ni11-Ti0.3 (AISI 321) & 1171 & 0.067 & 1.80 & 0.019 & & 0.013 & 0.54 & 0.121 \\
\hline $123 \mathrm{c}$ & Cr17-Nil1-Nb0.6 (AISI 348) & 1172 & 0.056 & $1.7_{5}$ & 0.024 & & 0.014 & 0.59 & 0.103 \\
\hline $133 \mathrm{~b}$ & Cr13-Mo0.3-S0.3 & & 0.128 & 1.07 & 0.018 & & 0.328 & 0.327 & 0.080 \\
\hline $160 \mathrm{~b}$ & Cr19-Ni12-Mo3 & 1155 & 0.044 & 1.64 & 0.020 & & 0.018 & $0.50_{9}$ & 0.172 \\
\hline $166 c$ & $\begin{array}{l}\text { Low Carbon (AISI 3162) } \\
\text { Carbon Only (100g unit) }\end{array}$ & & 0.0078 & & & & & & \\
\hline 339 & Cr17-Ni9-Se0.2 (SAE 303Se) & & 0.052 & 0.738 & 0.129 & & 0.013 & 0.654 & 0.199 \\
\hline 367 & Cr24-Ni0.3 (AISI 446) & 1267 & 0.093 & 0.315 & 0.018 & & 0.016 & 0.58 & \\
\hline
\end{tabular}


Stainless Steels (Continued)

\begin{tabular}{|c|c|c|c|c|c|c|c|c|c|c|c|}
\hline SRM & $\mathbf{N i}$ & $\mathrm{Cr}$ & $\mathrm{v}$ & Mo & Co & $\mathrm{Ti}$ & $\mathrm{Nb}$ & $\mathrm{Ta}$ & $\mathrm{Pb}$ & $\mathrm{Se}$ & $\mathbf{N}$ \\
\hline $73 c$ & 0.246 & 12.82 & 0.030 & 0.091 & & & & & & & 0.037 \\
\hline $121 \mathrm{~d}$ & 11.17 & $17.4_{3}$ & & 0.165 & 0.10 & 0.342 & & & & & \\
\hline $123 \mathrm{c}$ & $11.3_{4}$ & $17.4_{0}$ & & 0.22 & 0.12 & & 0.65 & $\langle 0.001$ & & & \\
\hline $133 b$ & 0.230 & 12.63 & 0.071 & 0.052 & & & & & & & \\
\hline $160 \mathrm{~b}$ & $12.2_{6}$ & $18.4_{5}$ & 0.047 & 2.38 & $0.10_{1}$ & & & & 0.001 & & $0.03_{9}$ \\
\hline \multicolumn{12}{|l|}{$166 \mathrm{c}$} \\
\hline 339 & 8.89 & 17.42 & 0.058 & 0.248 & 0.096 & & & & & 0.247 & \\
\hline 367 & 0.29 & 24.19 & 0.08 & & & & & & & & 0.168 \\
\hline
\end{tabular}

\section{Tool Steels}

\begin{tabular}{|c|c|c|c|c|c|c|c|c|c|c|c|}
\hline \multirow{3}{*}{ SRM } & \multirow{2}{*}{\multicolumn{2}{|c|}{ Type }} & & \multicolumn{8}{|c|}{ Chemical Composition (Nominal Weight Percent) } \\
\hline & & & & \multirow[t]{2}{*}{$\mathbf{C}$} & \multirow{2}{*}{\multicolumn{2}{|c|}{ Mn }} & \multirow[t]{2}{*}{$\mathbf{P}$} & \multicolumn{2}{|r|}{$\mathbf{S}$} & \multirow[t]{2}{*}{$\mathrm{Si}$} & \multirow[t]{2}{*}{$\mathrm{Cu}$} \\
\hline & & & & & & & & Grav & Comb & & \\
\hline $50 \mathrm{c}$ & \multicolumn{2}{|c|}{ W18-Cr4-V1 } & & 0.719 & & 0.342 & 0.022 & 0.010 & 0.009 & 0.311 & 0.079 \\
\hline $132 \mathrm{~b}$ & \multicolumn{2}{|c|}{ Mo-W.Cr-V } & & 0.864 & & 0.341 & 0.012 & & 0.004 & 0.185 & 0.008 \\
\hline $134 \mathrm{a}$ & \multirow{2}{*}{\multicolumn{2}{|c|}{$\begin{array}{l}\text { Mo8-W2-Cr4-V1 } \\
\text { Co8-Mo9-W2-Cr4-V2 }\end{array}$}} & & 0.808 & & 0.218 & 0.018 & 0.007 & 0.007 & 0.323 & 0.101 \\
\hline$\overline{153 a}$ & & & & 0.902 & & 0.192 & 0.023 & 0.007 & 0.007 & 0.270 & 0.094 \\
\hline SRM & $\mathrm{Ni}$ & $\mathrm{Cr}$ & $\mathbf{v}$ & & Mo & & $\mathbf{w}$ & Co & Sn & As & $\mathbf{N}$ \\
\hline $50 \mathrm{c}$ & 0.069 & 4.13 & 1.16 & & 0.082 & & 18.44 & & 0.018 & 0.022 & 0.012 \\
\hline $132 \mathrm{~b}$ & 0.230 & 4.38 & 1.83 & & 4.90 & & 6.28 & 0.029 & & & \\
\hline $134 \mathrm{a}$ & 0.088 & 3.67 & 1.25 & & 8.35 & & 2.00 & & & & \\
\hline $153 a$ & 0.168 & 3.72 & 2.06 & & 8.85 & & 1.76 & 8.47 & & & 0.024 \\
\hline
\end{tabular}

\section{Steels (Gramular Form)}

These granular-form SRM's are prepared by a pre-alloyed powder metallurgical process, which generally includes argon atomization and hydrogen annealing. The materials normally are sized between 0.07 to 0.7 $\mathrm{mm}$ to ensure satisfactory homgeneity and are issued in 100-gram units.

\begin{tabular}{|c|c|c|c|c|c|c|c|c|}
\hline \multirow[b]{2}{*}{ SRM } & \multirow[b]{2}{*}{ Туре } & \multicolumn{7}{|c|}{ Chemical Composition (Nominal Weight Percent) } \\
\hline & & C & Mn & $\mathbf{P}$ & $\mathbf{S}$ & Si & $\mathrm{Cu}$ & $\mathbf{N i}$ \\
\hline 163 & Low Alloy, $1.0 \mathrm{Cr}$ & 0.933 & 0.897 & 0.007 & 0.027 & 0.488 & 0.087 & 0.081 \\
\hline $101 \mathrm{f}$ & Stainless (AISI 304 L) & 0.014 & 0.087 & 0.008 & 0.008 & 0.876 & 0.030 & 9.96 \\
\hline
\end{tabular}




\section{Steels (Granular Form) (Continued)}

\begin{tabular}{lccccccccc}
\hline SRM & Cr & V & Mo & W & Co & N & As & Sb & Ga \\
\hline 163 & 0.982 & & 0.029 & & & 0.007 & & & \\
\hline $101 \mathrm{f}$ & 18.49 & 0.034 & 0.007 & $(0.0002)$ & 0.088 & & $(0.003)$ & $(0.0009)$ & $(0.004)$ \\
\hline
\end{tabular}

Values in parentheses are not certified, but are given for information only.

\section{Steels [Solid Form]}

Several groups of SRM's have been prepared to meet the basic needs of the steel industry for analytical control primarily by optical emission and x-ray spectroscopic methods of analysis. Both nominal composition and analytical range SRM's are provided for ingot iron, low-alloy steel, stainless steel, tool steel, and specialty steel.

These SRM's are furnished in various forms. The 400 series is intended for optical emission spectroscopic methods of analysis utilizing the "point-to-point" technique. The 600 series is intended for microchemical methods of analysis such as electron probe microanalysis, spark source mass spectrometric analysis, and laser probe analysis. The 800, 1100, and 1200 series are intended for "point-to-plane" optical emission spectroscopic methods of analysis. The D800 series, and the 1100 and 1200 series also are intended for $\mathrm{x}$-ray spectroscopic methods of analysis.

Because of the special homogeneity requirements, most of these materials have been prepared by using the most modern techniques of melting, casting, fabrication, and heat treatment to ensure adequate uniformity of composition.

Note: Values in parentheses are not certified as they are based on the results from a single laboratory. Values in brackets are not certified but are nominal values obtained from heat analyses. These values are given for additional information on the chemical composition.

\section{Nominal Sizes for Solid Steel SRM's}

400 Series: $5.5 \mathrm{~mm}(7 / 32 \mathrm{in})$ diameter, $102 \mathrm{~mm}(4 \mathrm{in})$ long

600 Series: $3.2 \mathrm{~mm}(1 / 8 \mathrm{in})$ diameter, $51 \mathrm{~mm}(2 \mathrm{in})$ long

800 Series: $13 \mathrm{~mm}(1 / 2 \mathrm{in})$ diameter, $51 \mathrm{~mm}(2 \mathrm{in})$ long

D800 Series: $31 \mathrm{~mm}(11 / 4 \mathrm{in})$ diameter, $6.4 \mathrm{~mm}(1 / 4$ in) thick

1100 and 1200 Series: $31 \mathrm{~mm}(11 / 4 \mathrm{in})$ diameter, $19 \mathrm{~mm}(3 / 4 \mathrm{in})$ thick

$\mathrm{C}$ indicates a chill cast sample: $31 \mathrm{~mm}(1 / / 4 \mathrm{in})$ diameter, $19 \mathrm{~mm}(3 / 4$ in) thick.

\section{Ingot Iron and Low-Alloy Steels}

The preparation of these original spectroscopic SRM's began in 1944 when the cores remaining after lathe cutting the materials for chip form standards were tested for homogeneity. Those found satisfactory were fabricated to the final shapes and sizes. To meet the urgent need in the mid-1950's for calibration standards for x-ray spectroscopic methods of analysis, portions of the material from five of these SRM's were converted to the applicable disk form. Although entirely satisfactory for conventional spectroscopic methods of analysis, these SRM's generally do not meet the stringent requirements for homogeneity necessary for use with the newer microchemical methods of analysis. These standards will be discontinued when the supply is exhausted. 


\section{Ingot Iron and Low-Alloy Steels}

\begin{tabular}{|c|c|c|c|c|c|c|c|c|}
\hline & \multirow[b]{2}{*}{ SRM } & & \multirow[b]{2}{*}{ Type } & \multicolumn{5}{|c|}{ Chemical Composition (Nominal Weight Percent) } \\
\hline & & & & Mn & Si & $\mathrm{Cu}$ & $\mathrm{Ni}$ & $\mathrm{Cr}$ \\
\hline & $803 a$ & D803a & Acid Open Hearth, 0.6C & 1.04 & 0.34 & 0.096 & 0.190 & 0.101 \\
\hline $404 \mathrm{a}$ & $804 a$ & & Basic Electric & 0.88 & 0.44 & 0.050 & 0.040 & 0.025 \\
\hline $405 \mathrm{a}$ & $805 a$ & & Medium Manganese & 1.90 & 0.27 & 0.032 & 0.065 & 0.37 \\
\hline $407 \mathrm{a}$ & $807 a$ & $\mathrm{D} 807 \mathrm{a}$ & Chromium-Vanadium & 0.76 & 0.29 & 0.132 & 0.169 & 0.92 \\
\hline $408 \mathrm{a}$ & $808 \mathrm{a}$ & & Chromium-Nickel & 0.76 & 0.28 & 0.10 & 1.20 & 0.655 \\
\hline $409 \mathrm{~b}$ & $809 b$ & & Nickel & 0.46 & 0.27 & 0.104 & 3.29 & 0.072 \\
\hline 413 & & & Acid Open Hearth, $0.4 \mathrm{C}$ & 0.67 & 0.22 & 0.25 & 0.18 & 0.055 \\
\hline 414 & & & Cr.Mo (SAE 4140) & 0.67 & 0.26 & 0.11 & 0.080 & 0.99 \\
\hline $417 \mathrm{a}$ & $817 \mathbf{a}$ & & Basic Open Hearth, $0.4 \mathrm{C}$ & 0.78 & & 0.13 & 0.062 & 0.050 \\
\hline $418 \mathrm{a}$ & & & Cr-Mo (SAE X4130) & 0.52 & 0.27 & 0.040 & 0.125 & 1.02 \\
\hline \multirow[t]{2}{*}{$\overline{420 \mathrm{a}}$} & $820 a$ & & Ingot Iron & 0.017 & & 0.027 & 0.0092 & 0.0032 \\
\hline & 821 & & $\mathrm{Cr}-\mathrm{W}, 0.9 \mathrm{C}$ & 1.24 & & 0.080 & 0.10 & 0.49 \\
\hline 427 & 827 & & Cr-Mo (SAE 4150)(B only) & & & & & \\
\hline
\end{tabular}

\begin{tabular}{|c|c|c|c|c|c|c|c|}
\hline & SRM & & V & Mo & Sn & $\underset{\text { (total) }}{\mathrm{Al}}$ & Other \\
\hline & $803 a$ & D803a & 0.005 & 0.033 & & & \\
\hline $404 a$ & $804 a$ & & 0.002 & 0.007 & & & \\
\hline $405 a$ & $805 a$ & & & 0.005 & & 0.056 & \\
\hline $407 a$ & $807 a$ & D807a & 0.146 & & & & \\
\hline $408 \mathrm{a}$ & $808 a$ & & 0.002 & 0.065 & & & \\
\hline$\overline{409 b}$ & $809 b$ & & 0.002 & 0.009 & 0.012 & & Co 0.025 \\
\hline 413 & & & 0.007 & 0.006 & & & \\
\hline 414 & & & 0.003 & 0.32 & 0.014 & 0.020 & \\
\hline $417 a$ & $817 a$ & & & 0.013 & 0.036 & & \\
\hline $418 \mathrm{a}$ & & & & 0.21 & & & \\
\hline \multirow[t]{2}{*}{$420 \mathrm{a}$} & $820 a$ & & & 0.0013 & 0.0017 & 0.003 & Co 0.006 \\
\hline & 821 & & 0.012 & 0.040 & & & W0.52 \\
\hline$\overline{427}$ & 827 & & & & & & B0.0027 \\
\hline
\end{tabular}




\section{Special Ingot Irons and Low-Alloy Steels}

The planning of the 1100 series SRM's began in late 1952 to meet critical requirements of calibration in the iron and steel industry. Steel for these SRM's was prepared by the most modern melting, casting, and fabrication techniques to provide large quantities of material of the highest possible homogeneity. The materials were fully characterized and included investigations by means of electron probe microanalysis and quantitative metallographic techniques. It was concluded that, for example, SRM's 461 and 463 are sufficiently homogeneous that any present microanalytical technique can be carried out with little chance of inaccuracy caused by inhomogeneity. Details of the metallographic and homogeneity characterization are given in NBS Miscellaneous Publication 260-3 and 260-10, respectively.

The 1200 series replaces the 1100 series which has been exhausted and consists of four low alloy steels and an electrolytic iron containing a graded series of 40 elements. Material from the same melts are available in three other forms: chip form, 361-365, for chemical methods of analysis; rods, $661-665,3.2 \mathrm{~mm}(1 / 8 \mathrm{in})$ in diameter and $51 \mathrm{~mm}(2 \mathrm{in})$ long for microchemical methods of analysis such as electron probe microanalysis, spark source mass spectrometric analysis, and laser probe analysis; and rods (1095-1099), $6.4 \mathrm{~mm}(1 / 4 \mathrm{in})$ in diameter and $102 \mathrm{~mm}$ (4 in) long for determining gases in metals by vacuum fusion and neutron activation methods of analysis. The preparation of the 1200 series involved a cooperative effort between industry and NBS, and represents the first application of the "benchmark" concept to SRM's. With thousands of industrial processes requiring analytical control, demands for SRM's far exceeded the NBS production capacity. An ever widening gap between supplies and demands led to a program to produce essential "benchmark" SRM's to serve as calibration points in measurement systems. While other selected low-alloy steel SRM's will be prepared to augment the 1200 series, this series is expected to be the primary "benchmarks," especially for some 25 trace elements that affect the physical properties of steels.

\section{Special Ingot Irons and Low-Alloy Steels}

\begin{tabular}{|c|c|c|c|c|c|c|c|c|}
\hline \multirow{2}{*}{\multicolumn{2}{|c|}{ SRM }} & \multirow[b]{2}{*}{ Type } & \multirow[b]{2}{*}{ (Other Forms) } & \multicolumn{5}{|c|}{ Chemical Composition (Nominal Weight Percent) } \\
\hline & & & & $\mathrm{C}$ & Mn & $\mathbf{P}$ & $\mathbf{S}$ & Si \\
\hline & 1134 & High-Silicon & $125 b$ & 0.026 & 0.277 & 0.028 & 0.009 & 2.89 \\
\hline & 1135 & High-Silicon & 179 & 0.027 & 0.094 & 0.006 & 0.026 & 3.19 \\
\hline & 1136 & High-Silicon & $129 \mathrm{c}$ & $0.11_{3}$ & $0.75_{5}$ & 0.066 & 0.220 & 0.018 \\
\hline$\overline{461}$ & & Low Alloy A & & 0.15 & 0.36 & 0.053 & 0.019 & 0.047 \\
\hline 462 & & Low Alloy B & & 0.40 & 0.94 & 0.045 & 0.019 & 0.28 \\
\hline 464 & & Low Alloy D & & 0.54 & 1.32 & 0.017 & 0.021 & 0.48 \\
\hline 465 & & Ingot Iron $\mathrm{E}$ & & 0.037 & 0.032 & 0.008 & 0.01 & 0.029 \\
\hline 466 & 1166 & Ingot Iron F & & 0.065 & $0.11_{3}$ & 0.012 & 0.009 & 0.025 \\
\hline 467 & & Low Alloy $G$ & & 0.11 & $0.27_{5}$ & 0.033 & 0.009 & 0.26 \\
\hline 468 & & Low Alloy $\mathrm{H}$ & & 0.26 & 0.47 & 0.023 & 0.020 & 0.075 \\
\hline & $1169 \mathrm{~b}$ & Lead-Bearing & & 0.1 & $(1.1)$ & $(0.07)$ & $(0.3)$ & $(0.01)$ \\
\hline & $\mathrm{C} 1221$ & Resulfurized/Rephosphorized & & 0.020 & 0.102 & 0.090 & 0.112 & 0.876 \\
\hline & 1222 & Cr-Ni-Mo (AISI 8640) & $139 b$ & 0.43 & 0.78 & 0.013 & 0.022 & 0.24 \\
\hline & 1224 & Carbon & & 0.75 & 0.41 & 0.009 & 0.039 & 0.173 \\
\hline & 1225 & Low Alloy (AISI 4130) & & 0.274 & 0.48 & 0.007 & 0.014 & 0.221 \\
\hline & 1226 & Low Alloy & & 0.085 & 0.274 & 0.0022 & 0.0044 & 0.231 \\
\hline & 1227 & Basic Open Hearth, $1 \%$ & & 0.97 & 0.402 & 0.014 & 0.026 & 0.215 \\
\hline & 1228 & Basic Open Hearth, $0.1 \%$ & & 0.072 & 0.365 & 0.004 & 0.018 & 0.007 \\
\hline & 1254 & $\mathrm{Ca}$ in Low Alloy (Si) & & $(0.03)$ & $(0.28)$ & $(0.03)$ & $(0.008)$ & $(2.9)$ \\
\hline$* 661$ & $1261 \mathrm{a}$ & AISI 4340 & 361,1095 & 0.39 & 0.66 & 0.015 & 0.015 & 0.223 \\
\hline
\end{tabular}




\section{Special Ingot Irons and Low-Alloy Steels (Continued)}

\begin{tabular}{|c|c|c|c|c|c|c|c|c|}
\hline \multirow[b]{2}{*}{ SRM } & & \multirow[b]{2}{*}{ Type } & \multirow[b]{2}{*}{ (Other Forms) } & \multicolumn{5}{|c|}{ Chemical Composition (Nominal Weight Percent) } \\
\hline & & & & $\mathrm{C}$ & Mn & $\mathbf{P}$ & $\mathbf{S}$ & $\mathbf{S i}$ \\
\hline *662 & $1262 \mathrm{a}$ & AISI 94B17 (Mod) & 362,1096 & 0.163 & 1.05 & 0.044 & 0.037 & 0.40 \\
\hline *663 & $1263 \mathrm{a}$ & $\mathrm{Cr}-\mathrm{V}$ (Mod) & 363,1097 & 0.57 & 1.50 & $0.02_{9}$ & 0.0055 & 0.74 \\
\hline *664 & $1264 \mathrm{a}$ & High Carbon (Mod) & 364,1098 & 0.871 & $0.25_{8}$ & 0.010 & 0.025 & 0.066 \\
\hline \multirow[t]{5}{*}{ *665 } & $1265 \mathrm{a}$ & Electrolytic Iron. & 365,1099 & 0.008 & 0.0057 & $0.002_{5}$ & 0.0059 & 0.0080 \\
\hline & 1269 & Low Alloy (AISI 1526, Mod) & & 0.298 & 1.35 & 0.012 & 0.0061 & 0.189 \\
\hline & 1270 & Cr-Mo Low Alloy & & 0.077 & 0.626 & 0.0065 & 0.0065 & 0.247 \\
\hline & C1285 & Low Alloy (A242 Mod) & & 0.058 & 0.332 & 0.072 & 0.020 & 0.36 \\
\hline & 1286 & Low Alloy (Hy 80) & & 0.196 & 0.152 & 0.008 & 0.017 & 0.130 \\
\hline
\end{tabular}

\begin{tabular}{|c|c|c|c|c|c|c|c|c|c|}
\hline \multicolumn{2}{|c|}{ SRM } & \multirow{2}{*}{$\begin{array}{c}\mathrm{Cu} \\
0.070\end{array}$} & \multirow{2}{*}{$\begin{array}{c}\mathrm{Ni} \\
0.038\end{array}$} & \multirow{2}{*}{$\begin{array}{c}\mathbf{C r} \\
0.019\end{array}$} & \multirow[t]{2}{*}{ V } & \multirow{2}{*}{$\begin{array}{c}\text { Mo } \\
0.008 \\
\end{array}$} & \multirow[t]{2}{*}{ W } & \multirow[t]{2}{*}{ Co } & \multirow[t]{2}{*}{$\mathrm{Ti}$} \\
\hline & 1134 & & & & & & & & \\
\hline & 1135 & 0.056 & 0.050 & 0.022 & $\langle 0.01$ & 0.014 & & & \\
\hline & 1136 & 0.014 & 0.270 & 0.014 & 0.012 & 0.002 & & & \\
\hline 461 & & 0.34 & 1.73 & 0.13 & 0.024 & 0.30 & 0.012 & 0.26 & $(0.01)$ \\
\hline 462 & & 0.20 & 0.70 & 0.74 & 0.058 & 0.080 & 0.053 & 0.11 & 0.037 \\
\hline 464 & & 0.094 & 0.135 & 0.078 & $0.29_{5}$ & 0.029 & 0.022 & $0.02_{8}$ & 0.004 \\
\hline 465 & & 0.019 & 0.026 & 0.004 & 0.002 & 0.005 & $(0.001)$ & 0.008 & 0.20 \\
\hline 466 & & 0.033 & 0.051 & 0.011 & 0.007 & 0.011 & $(0.006)$ & $0.04_{6}$ & 0.057 \\
\hline 467 & & 0.067 & 0.088 & 0.036 & 0.041 & 0.021 & 0.20 & $0.07_{4}$ & 0.26 \\
\hline \multirow[t]{10}{*}{468} & & 0.26 & 1.03 & 0.54 & 0.17 & 0.20 & 0.077 & 0.16 & 0.011 \\
\hline & $1169 \mathrm{~b}$ & $(0.07)$ & $(0.04)$ & $(0.05)$ & $(0.001)$ & $(0.01)$ & & & \\
\hline & $\mathrm{C} 1221$ & 0.041 & 0.067 & 0.049 & $(0.0007)$ & 0.038 & & $(0.010)$ & $(0.0014)$ \\
\hline & 1222 & 0.097 & 0.51 & 0.48 & 0.005 & 0.18 & & $(0.016)$ & $(0.002)$ \\
\hline & 1224 & 0.072 & 0.054 & 0.071 & 0.002 & 0.013 & & & \\
\hline & 1225 & & 0.018 & 0.91 & 0.004 & 0.166 & & & \\
\hline & 1226 & 0.125 & 5.42 & 0.467 & 0.0018 & 0.446 & $(0.005)$ & 0.029 & 0.0021 \\
\hline & 1227 & 0.006 & 0.007 & 0.019 & 0.002 & 0.003 & & 0.003 & $(0.0008)$ \\
\hline & 1228 & 0.012 & 0.018 & 0.016 & $<0.001$ & 0.009 & & & \\
\hline & 1254 & $(0.07)$ & $(0.04)$ & $(0.02)$ & & $(0.008)$ & & & \\
\hline$\overline{* 661}$ & $1261 \mathrm{a}$ & 0.042 & 1.99 & 0.69 & 0.011 & 0.19 & 0.017 & 0.032 & 0.020 \\
\hline${ }^{* 662}$ & $1262 a$ & 0.51 & 0.60 & 0.30 & 0.04 & $0.07_{0}$ & 0.21 & 0.30 & 0.084 \\
\hline${ }^{* 663}$ & $1263 a$ & 0.098 & 0.32 & 1.31 & 0.31 & 0.030 & 0.046 & 0.048 & 0.050 \\
\hline${ }^{*} 664$ & $1264 a$ & 0.250 & 0.142 & $0.06_{6}$ & $0.10_{6}$ & 0.49 & $0.10_{2}$ & 0.15 & 0.23 \\
\hline \multirow[t]{5}{*}{$* 665$} & $1265 \mathrm{a}$ & 0.0058 & 0.041 & $0.007_{2}$ & 0.0006 & 0.005 & $(0.0004)$ & $0.007_{0}$ & 0.0006 \\
\hline & 1269 & 0.095 & 0.108 & 0.201 & 0.004 & 0.036 & $(0.001)$ & $(0.014)$ & $(0.009)$ \\
\hline & 1270 & 0.114 & 0.174 & 2.34 & 0.013 & 0.956 & $(0.003)$ & 0.038 & $(0.003)$ \\
\hline & $\mathrm{C} 1285$ & 0.37 & 1.17 & 0.80 & & & & & \\
\hline & 1286 & 0.043 & 2.81 & 1.53 & & & & & \\
\hline
\end{tabular}


Special Ingot Irons and Low-Alloy Steels (Continued)

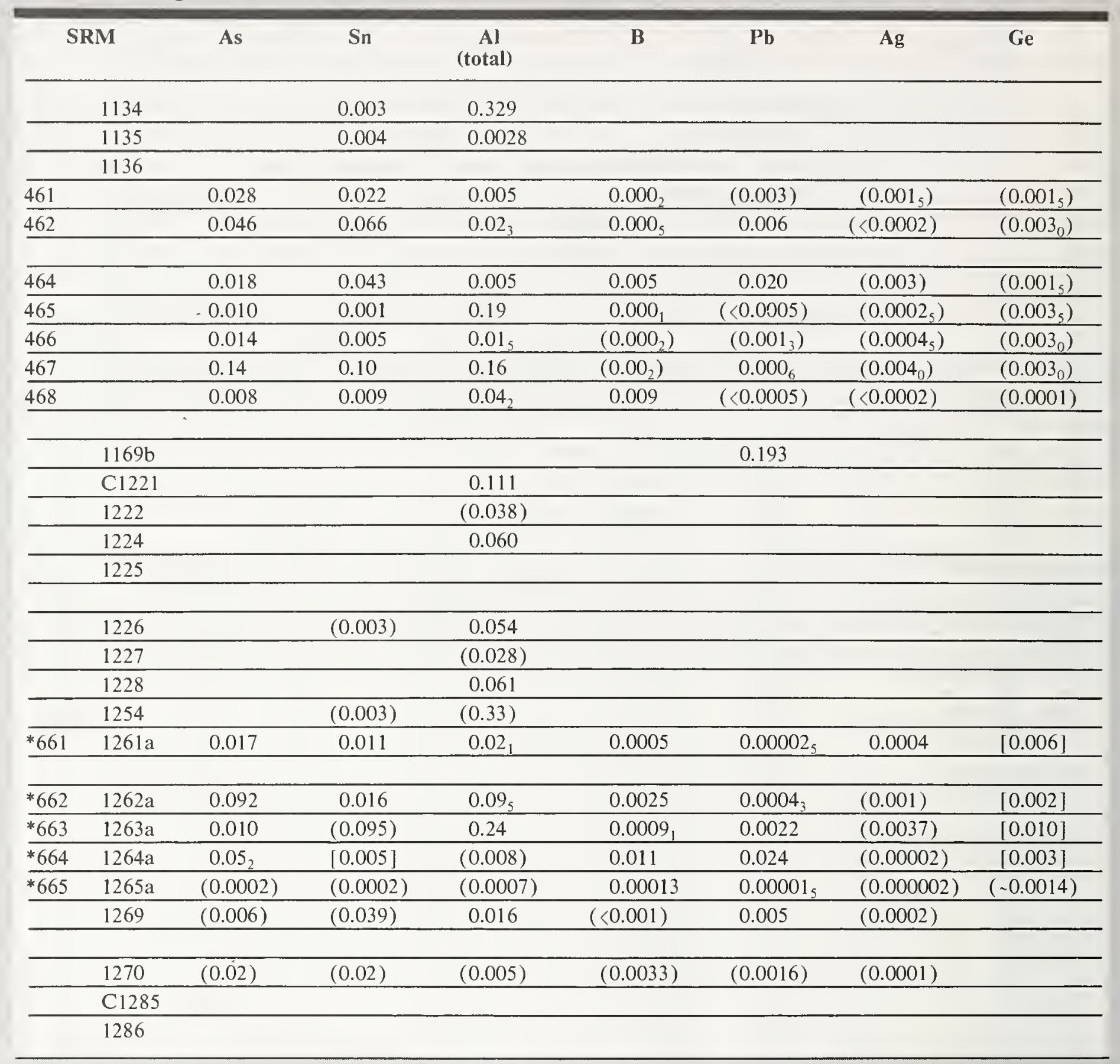


Special Ingot Irons and Low-Alloy Steels (Continued)

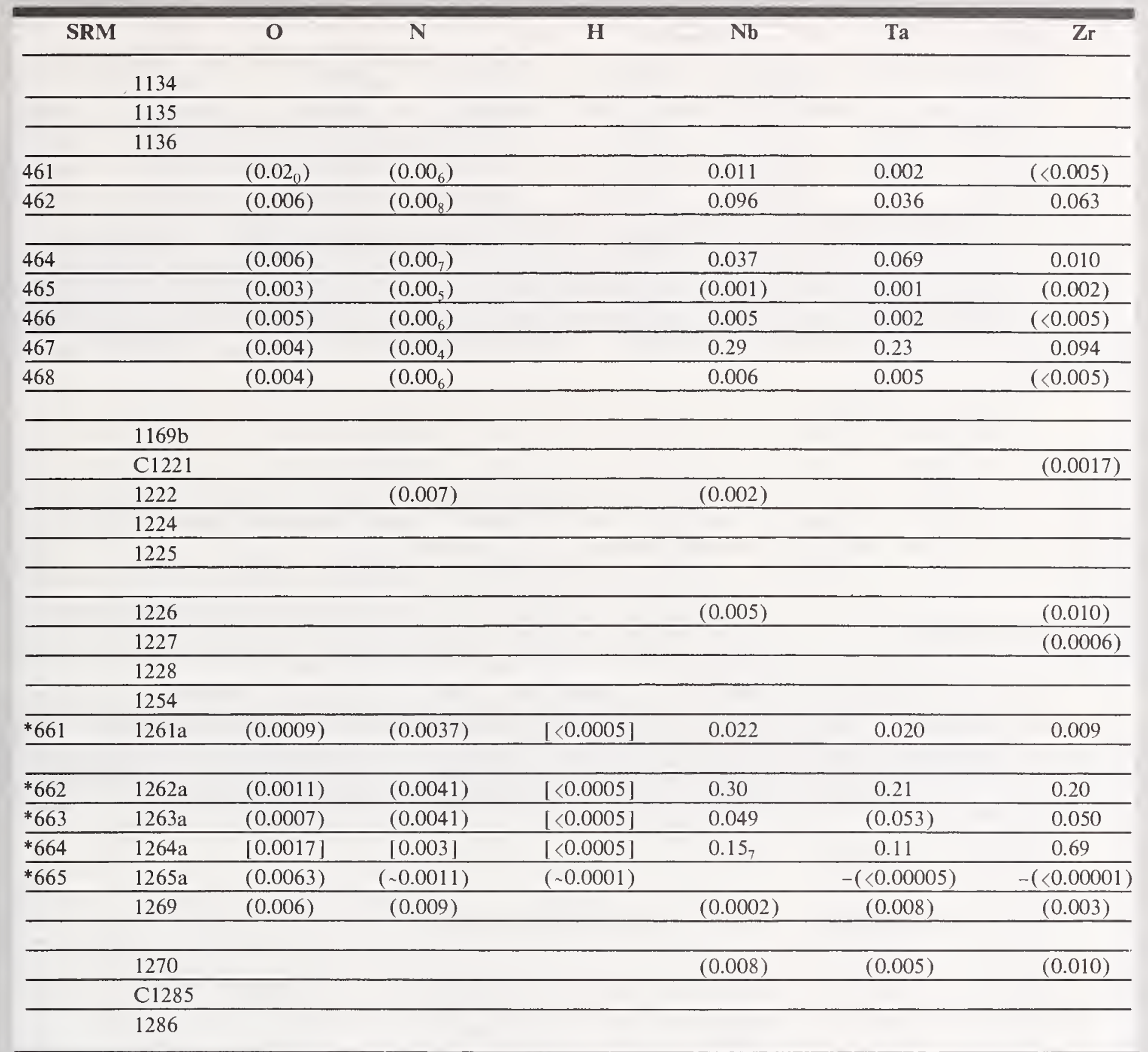

\begin{tabular}{cccccccc}
\hline \multicolumn{2}{c}{ SRM } & Sb & Bi & Ca & Mg & Te & Zn \\
\hline$* 661$ & $1261 \mathrm{a}$ & 0.0042 & 0.0004 & $(<0.0001)$ & $(0.0001)$ & 0.006 & $(0.0001)$ \\
\hline$* 662$ & $1262 \mathrm{a}$ & $0.012_{0}$ & $(0.002)$ & $(0.0002)$ & $(0.0006)$ & $(0.0005)$ & $(0.0005)$ \\
\hline$* 663$ & $1263 \mathrm{a}$ & 0.002 & $(0.0008)$ & $(<0.0001)$ & $(0.0005)$ & $(0.0022)$ & $(0.0004)$ \\
\hline$* 664$ & $1264 \mathrm{a}$ & $(0.035)$ & $(0.0009)$ & $(<0.0001)$ & $(0.0001)$ & {$[0.0002]$} & {$[0.001]$} \\
\hline${ }^{*} 665$ & $1265 \mathrm{a}$ & $-(<0.00005)$ & $-(<0.00001)$ & $-(<0.00001)$ & $-(<0.00002)$ & $-(<0.00001)$ & $(<0.0001)$ \\
\hline
\end{tabular}


Special Ingot Irons and Low-Alloy Steels (Continued)

\begin{tabular}{|ccccccccc}
\hline \multicolumn{2}{c}{ SRM } & Au & Ce & Hf & La & Nd & Pr & Fe \\
\hline${ }^{* 661}$ & $1261 \mathrm{a}$ & $(<0.00005)$ & 0.0013 & {$[0.0002]$} & 0.0004 & 0.0003 & $(0.00014)$ & $(95.6)$ \\
\hline${ }^{* 662}$ & $1262 \mathrm{a}$ & $(<0.00005)$ & $(0.0011)$ & {$[0.006]$} & 0.0004 & $(0.0005)$ & $(0.00012)$ & $(95.3)$ \\
\hline${ }^{* 663}$ & $1263 \mathrm{a}$ & 0.0005 & $(0.0016)$ & {$[0.0015]$} & 0.0006 & $(0.0007)$ & $(0.00018)$ & $(94.4)$ \\
\hline${ }^{* 664}$ & $1264 \mathrm{a}$ & 0.0001 & $(0.00025)$ & {$[0.005]$} & 0.00007 & $(0.00012)$ & $(0.00003)$ & $(96.7)$ \\
\hline${ }^{*} 665$ & $1265 \mathrm{a}$ & $-(<0.000002)$ & $-(<0.000005)$ & $-(<0.00002)$ & $-(<0.000005)$ & $-(<0.000005)$ & $-(<0.000005)$ & $(99.9)$ \\
\hline
\end{tabular}

*SRM's 661, 662, 663, 664, and 665 are sold in a set only as SRM S668.

Values in parentheses are not certified, but are given for information only.

Brackets indicate approximate value from heat analysis.

Dash indicates "not detected." Value in parentheses following dash is the conservative "upper limit" of detection.

\section{Stainless Steels}

Three groups of stainless steel SRM's designed primarily for calibration in spectroscopic methods of analysis are available.

Groups I and II have been extensively tested for homogeneity and found satisfactory for application in conventional spectroscopic methods of analysis. Neither group, however, has been tested for microanalytical methods and their use in these applications is not recommended.

Group III are for the "point-to-plane" technique of emission spectroscopy and for x-ray spectroscopy. They were prepared by melting, casting, and fabrication techniques known to produce material of high homogeneity.

\begin{tabular}{|c|c|c|c|c|c|c|c|c|c|c|c|}
\hline \multicolumn{12}{|c|}{ Group II } \\
\hline \multirow[b]{2}{*}{ SRM } & \multirow[b]{2}{*}{ Type } & & \multicolumn{9}{|c|}{ Chemical Composition (Nominal Weight Percent) } \\
\hline & & & $\mathbf{M n}$ & $\mathrm{Si}$ & $\mathrm{Cu}$ & $\mathrm{Ni}$ & $\mathrm{Cr}$ & $\mathbf{V}$ & Mo & W & $\mathrm{Co}$ \\
\hline 442 & Cr16-Ni10 & & 2.88 & $(0.09)$ & 0.11 & 9.9 & 16.1 & 0.032 & 0.12 & $(0.08)$ & 0.13 \\
\hline 443 & Cr18.5-Ni9.5 & & 3.38 & $(0.15)$ & 0.14 & 9.4 & 18.5 & 0.064 & 0.12 & $(0.09)$ & 0.12 \\
\hline$\overline{444}$ & $\mathrm{Cr} 20.5-\mathrm{Ni} 10$ & & 4.62 & $(0.65)$ & 0.24 & 10.1 & 20.5 & 0.12 & 0.23 & $(0.17)$ & 0.22 \\
\hline SRM & $\mathrm{Ti}$ & Sn & & $\mathrm{Nb}$ & $\mathrm{Ta}$ & & & $\mathrm{Pb}$ & $\mathrm{Zr}$ & & $\mathrm{Zn}$ \\
\hline 442 & 0.002 & 0.0035 & & 0.032 & $(0.0006)$ & & & 0.0017 & $(0.004)$ & & $(0.003)$ \\
\hline 443 & 0.003 & 0.006 & & 0.056 & $(0.0008)$ & & & 0.0025 & & & $(0.005)$ \\
\hline$\overline{444}$ & 0.019 & 0.014 & & 0.20 & $(0.004)$ & & & 0.0037 & $(0.11)$ & & $(0.004)$ \\
\hline
\end{tabular}

Values in parentheses are not certified, but are given for information only. 


\section{Group II}

\begin{tabular}{|c|c|c|c|c|c|c|c|c|c|}
\hline & \multirow{2}{*}{\multicolumn{2}{|c|}{ SRM }} & \multirow[b]{2}{*}{ Type } & \multicolumn{6}{|c|}{ Chemical Composition (Nominal Weight Percent) } \\
\hline & & & & Mn & $\mathrm{Si}$ & $\mathrm{Cu}$ & $\mathrm{Ni}$ & $\mathrm{Cr}$ & v \\
\hline 445 & & & Cr13-Mo0.9 (Mod. AISI 410) & 0.77 & 0.52 & 0.065 & 0.28 & 13.31 & $(0.05)$ \\
\hline 446 & & & Cr18-Ni9 (Mod. AISI 321) & 0.53 & 1.19 & 0.19 & 9.11 & 18.35 & $(0.03)$ \\
\hline 447 & & & Cr24-Ni13 (Mod. AISI 309) & 0.23 & 0.37 & 0.19 & 13.26 & 23.72 & $(0.03)$ \\
\hline 448 & & & Cr9-Mo0.3 (Mod. AISI 403) & 2.13 & 1.25 & 0.16 & 0.52 & 9.09 & $(0.02)$ \\
\hline 449 & 849 & D849 & Cr5.5-Ni6.5 & 1.63 & 0.68 & 0.21 & 6.62 & 5.48 & $(0.01)$ \\
\hline 450 & 850 & D850 & $\mathrm{Cr} 3-\mathrm{Ni} 25$ & & 0.12 & 0.36 & 24.8 & 2.99 & $(0.006)$ \\
\hline
\end{tabular}

\begin{tabular}{|c|c|c|c|c|c|c|c|c|}
\hline & SRM & & Mo & $\mathbf{W}$ & $\mathrm{Ti}$ & Sn & $\mathbf{N b}$ & Ta \\
\hline 445 & & & 0.92 & $(0.42)$ & $(0.03)$ & & 0.11 & $(0.002)$ \\
\hline 446 & & & 0.43 & $(0.04)$ & $(0.34)$ & $(0.02)$ & 0.60 & $(0.030)$ \\
\hline 447 & & & 0.059 & $(0.06)$ & $(0.02)$ & & 0.03 & $(0.002)$ \\
\hline 448 & & & 0.33 & $(0.14)$ & $(0.23)$ & $(0.05)$ & 0.49 & $(0.026)$ \\
\hline 449 & 849 & D849 & 0.15 & $(0.19)$ & $(0.11)$ & $(0.07)$ & 0.31 & $(0.021)$ \\
\hline$\overline{450}$ & 850 & D850 & & $(0.21)$ & $(0.05)$ & $(0.09)$ & 0.05 & $(0.002)$ \\
\hline
\end{tabular}

Values in parentheses are not certified, but are given for information only.

\section{Group III}

\begin{tabular}{|c|c|c|c|c|c|c|c|c|c|c|}
\hline \multirow[b]{2}{*}{ SRM } & \multirow[b]{2}{*}{ Type } & \multirow[b]{2}{*}{$\begin{array}{l}\text { Other } \\
\text { Forms }\end{array}$} & \multicolumn{8}{|c|}{ Chemical Composition (Nominal Weight Percent) } \\
\hline & & & $\mathrm{C}$ & Mn & $\mathbf{P}$ & $\mathrm{S}$ & $\mathrm{Si}$ & $\mathrm{Cu}$ & $\mathbf{N i}$ & $\mathrm{Cr}$ \\
\hline $1151 \mathrm{a}$ & Cr22-Ni7-IN PREP & & & & & & & & & \\
\hline C1151 & $\mathrm{Cr} 22 \cdot \mathrm{Ni} 7$ & & 0.039 & 2.50 & 0.017 & 0.038 & 0.38 & 0.418 & 7.29 & 22.70 \\
\hline $1152 \mathrm{a}$ & Cr18-Ni10-IN PREP & & & & & & & & & \\
\hline C1152 & Crl8-Nil0 & & 0.148 & 0.96 & 0.021 & 0.0064 & 0.80 & 0.102 & 10.88 & 17.81 \\
\hline $1153 \mathrm{a}$ & Cr16-Ni8-IN PREP & & & & & & & & & \\
\hline C1153 & Cr16-Ni8 & & 0.264 & 0.50 & 0.030 & 0.018 & 1.07 & 0.23 & 8.77 & 16.69 \\
\hline $1154 a$ & Cr19-Ni12-IN PREP & & & & & & & & & \\
\hline C1154 & Cr19.Ni12 & & 0.086 & 1.42 & 0.06 & 0.053 & 0.50 & 0.40 & 12.92 & 19.06 \\
\hline 1155 & Cr18-Ni12-Mo2 (AISI 3 & 16) $160 b$ & 0.046 & 1.63 & 0.020 & 0.018 & 0.502 & 0.169 & 12.18 & 18.45 \\
\hline $1170 \mathrm{~b}$ & Selenium-Bearing & & $(0.052)$ & $(0.738)$ & $(0.129)$ & $(0.013)$ & $(0.654)$ & $(0.199)$ & $(8.89)$ & $(17.42)$ \\
\hline 1171 & Cr17-Nil1-Ti0.3 & $121 \mathrm{~d}$ & 0.067 & $1.8_{0}$ & 0.018 & $0.01_{3}$ & 0.54 & 0.121 & 11.2 & 17.4 \\
\hline 1172 & Cr17-Nil1-Nb0.6 & $123 c$ & 0.056 & $1.7_{6}$ & 0.025 & $0.01_{4}$ & 0.59 & $0.10_{5}$ & $11.3_{5}$ & $17.4_{0}$ \\
\hline 1267 & AISI 446 & 367 & 0.093 & 0.315 & 0.018 & 0.015 & 0.58 & & 0.29 & 24.14 \\
\hline C1287 & AISI 310 Mod. & & 0.36 & 1.66 & 0.029 & 0.024 & 1.66 & 0.58 & 21.16 & 23.98 \\
\hline C1288 & (A-743) & & 0.056 & 0.83 & 0.023 & 0.010 & 0.41 & 3.72 & 29.3 & 19.55 \\
\hline C1289 & AISI 414 Mod. & & 0.014 & 0.35 & 0.017 & 0.021 & 0.156 & 0.205 & 4.13 & 12.12 \\
\hline
\end{tabular}




\section{Group III (Continued)}

\begin{tabular}{|c|c|c|c|c|c|c|c|c|c|c|c|c|}
\hline SRM & V & Mo & Co & $\mathrm{Ti}$ & As & Sn & $\mathrm{Al}$ & $\mathrm{Nb}$ & $\mathrm{Ta}$ & B & $\mathrm{Pb}$ & $\mathrm{Zr}$ \\
\hline \multicolumn{13}{|l|}{$1151 \mathrm{a}$} \\
\hline $\mathrm{C} 1151$ & 0.037 & 0.80 & 0.032 & & & & & & & & 0.0039 & \\
\hline \multicolumn{13}{|l|}{$1152 a$} \\
\hline C1152 & 0.030 & 0.43 & 0.22 & & & & & & & & 0.0047 & \\
\hline \multicolumn{13}{|l|}{$1153 \mathrm{a}$} \\
\hline C1153 & 0.18 & 0.24 & 0.127 & & & & & & & & 0.0054 & \\
\hline \multicolumn{13}{|l|}{$1154 a$} \\
\hline$\underline{\mathrm{C} 1154}$ & 0.135 & 0.07 & 0.38 & & & & & & & & 0.0178 & \\
\hline 1155 & 0.047 & 2.38 & 0.101 & & & & & & & & 0.001 & \\
\hline $1170 \mathrm{~b}$ & $(0.058)$ & $(0.248)$ & $(0.096)$ & & & & & & & & & Se 0.23 \\
\hline 1171 & & $0.16_{5}$ & 0.10 & 0.34 & & & & & & & & \\
\hline 1172 & & 0.22 & 0.12 & & & & & 0.65 & $<0.001$ & & & \\
\hline 1267 & 0.08 & & & & & & & & & & & N 0.17 \\
\hline $\mathrm{C} 1287$ & 0.09 & 0.46 & 0.31 & 0.050 & & $\mathrm{~N}(0.034)$ & $(0.06)$ & $(0.07)$ & $\mathrm{O}(0.017)$ & & 0.008 & $(0.006)$ \\
\hline $\mathrm{C} 1288$ & 0.086 & 2.83 & 0.10 & 0.012 & & $\mathrm{~N}(0.028)$ & $(0.0025)$ & $(0.22)$ & $\mathrm{O}(0.029)$ & $\mathrm{W}(0.2)$ & 0.0041 & $(0.002)$ \\
\hline $\mathrm{C} 1289$ & 0.007 & 0.82 & 0.035 & 0.005 & & $\mathrm{~N}(0.017)$ & $(0.0016)$ & $(0.10)$ & $\mathrm{O}(0.027)$ & & 0.0005 & $(0.001)$ \\
\hline
\end{tabular}

Values in parentheses are not certified, but are given for information only.

\section{Specialty Steels}

SRM's 1156, Maraging Steel, and 1158, High-Nickel Steel (Invar), are designed primarily for use in optical emission and $\mathrm{x}$-ray spectrometric methods of analysis.

SRM 1156 derives its name from the formation of martensite on age hardening. Alloys of this type are used extensively in submarines, missiles, and aircraft.

SRM 1158 has good impact toughness down to $-269^{\circ} \mathrm{C}$ and has an extremely low coefficient of expansion between -253 and $203{ }^{\circ} \mathrm{C}$. These properties make this material very useful for cryogenic application. SRM 1158 also serves as a "benchmark" for the production control of high-nickel (40Ni-60Fe) alloys.

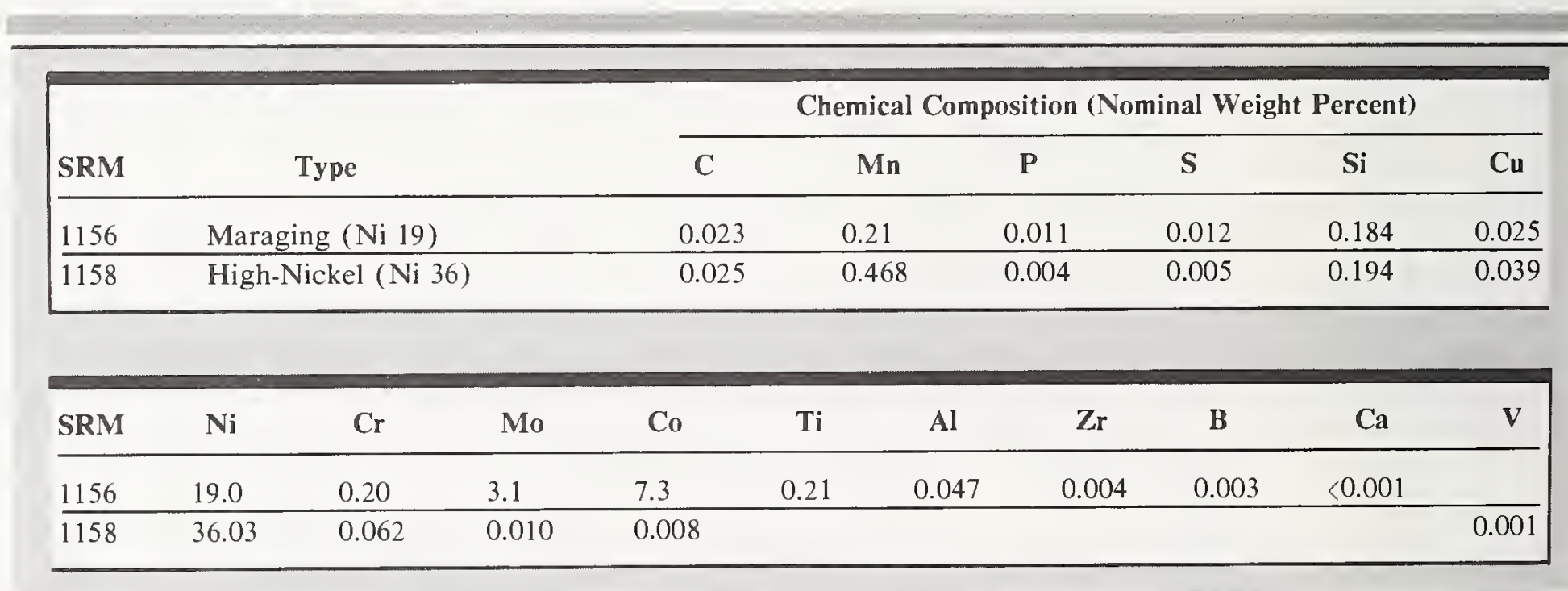




\section{High-Temperature Alloys}

High-temperature alloy SRM's were prepared to meet the critical needs of industry, particularly the aerospace industry, and government agencies. These SRM's are useful in instrument calibration, primarily for $\mathrm{x}$-ray and optical emission spectroscopic methods of analysis.

\begin{tabular}{|c|c|c|c|c|c|c|c|}
\hline \multirow[b]{2}{*}{ SRM } & \multirow[b]{2}{*}{ Type } & \multicolumn{6}{|c|}{ Chemical Composition (Nominal Weight Percent) } \\
\hline & & $\mathrm{C}$ & Mn & $\mathbf{P}$ & $\mathbf{S}$ & Si & $\mathrm{Cu}$ \\
\hline 1197 & M308 & $(0.045)$ & $(0.047)$ & $(0.008)$ & $(0.002)$ & $(0.13)$ & \\
\hline \multirow[t]{2}{*}{$\mathbf{S 1 1 9 8}$} & Incoloy 901 set & $(0.048)$ & $(0.49)$ & $(0.006)$ & $(0.002)$ & $(0.38)$ & $(0.012)$ \\
\hline & Hastelloy X & $(0.039)$ & & $(0.008)$ & & $(0.54)$ & \\
\hline \multirow[t]{2}{*}{ S1199 } & L 605 set & $(0.14)$ & 1.42 & $(0.005)$ & & 0.83 & \\
\hline & S 816 & $(0.40)$ & 1.34 & $(0.015)$ & & 0.86 & \\
\hline $1206-2$ & René-4l & $0.21_{7}$ & 0.030 & $(0.004)$ & 0.006 & $0.21_{6}$ & 0.040 \\
\hline $1207-1$ & Waspaloy (1) & 0.043 & 0.34 & 0.005 & 0.009 & $0.47_{2}$ & 0.026 \\
\hline 1207.2 & Waspaloy (2) & 0.083 & $0.29_{5}$ & 0.005 & 0.009 & $0.61_{5}$ & 0.033 \\
\hline $1208-1$ & Inco $718(1)$ & 0.046 & $0.38_{5}$ & 0.003 & $0.01_{1}$ & $0.43_{4}$ & $0.14_{7}$ \\
\hline $1208-2$ & Inco $718(2)$ & 0.022 & $0.23_{0}$ & 0.003 & 0.007 & $0.08_{3}$ & 0.077 \\
\hline 1244 & Inconel 600 & 0.06 & 0.3 & $<0.01$ & $<0.01$ & 0.1 & 0.3 \\
\hline 1245 & Inconel 625 & 0.04 & 0.2 & 0.01 & $<0.01$ & 0.4 & 0.4 \\
\hline 1246 & Incoloy 800 & 0.08 & 0.9 & 0.02 & $<0.01$ & 0.1 & 0.5 \\
\hline 1247 & Incoloy 825 & 0.02 & 0.4 & 0.02 & $<0.01$ & 0.3 & 1.6 \\
\hline
\end{tabular}

\begin{tabular}{|c|c|c|c|c|c|c|c|c|c|c|c|}
\hline SRM & $\mathbf{N i}$ & $\mathrm{Cr}$ & Mo & $\mathrm{Co}$ & $\mathrm{Ti}$ & $\mathbf{A l}$ & $\mathrm{Nb}$ & $\mathrm{Ta}$ & $\mathrm{Fe}$ & W & $\mathbf{B}$ \\
\hline 1197 & 32.6 & 12.9 & $3.2_{5}$ & $(0.07)$ & 2.32 & 0.41 & $(<0.02)$ & Zr0.15 & 41.9 & $6.0_{8}$ & $(0.0059)$ \\
\hline \multirow[t]{2}{*}{ S1198 } & 40.1 & 12.9 & $6.0_{8}$ & 0.70 & 2.59 & 0.24 & $(<0.02)$ & $\operatorname{Zr}(0.014)$ & 36.2 & $(0.2)$ & $(0.0064)$ \\
\hline & 45.7 & 20.7 & $9.1_{8}$ & 0.56 & $(<0.01)$ & & $(<0.02)$ & & 23.2 & $(0.15)$ & \\
\hline \multirow[t]{2}{*}{ S1199 } & 10.2 & 19.9 & $(<0.02)$ & 51.6 & $(<0.01)$ & & $(<0.02)$ & & $0.6_{5}$ & 15.4 & \\
\hline & 20.0 & 19.9 & $4.0_{0}$ & 42.0 & $(0.03)$ & & $3.1_{8}$ & 1.08 & 3.19 & $3.8_{6}$ & \\
\hline 1206.2 & 53.3 & 19.7 & $10.3_{0}$ & $11.5_{5}$ & $2.9_{4}$ & $1.7_{4}$ & & & 0.46 & & \\
\hline $1207-1$ & 56.1 & 18.88 & 4.50 & $13.0_{5}$ & 3.09 & 1.26 & & & 2.22 & & \\
\hline $1207-2$ & 55.7 & $19.4_{4}$ & 4.34 & $13.5_{0}$ & 2.54 & $1.3_{9}$ & & & 2.09 & & \\
\hline $1208-1$ & 51.9 & 17.5 & $3.2_{4}$ & 0.82 & 0.46 & $(0.15)$ & $5.3_{8}$ & $(0.012)$ & 19.2 & & \\
\hline $1208-2$ & 51.5 & 17.4 & 3.13 & 0.76 & $\left(0.8_{5}\right)$ & $\left(0.8_{5}\right)$ & $4.9_{8}$ & $(0.012)$ & 19.8 & & \\
\hline 1244 & 72.6 & 16 & 0.2 & 0.06 & 0.3 & 0.3 & 0.1 & & 9 & & $<0.01$ \\
\hline 1245 & 60 & 22 & 8.7 & 0.1 & 0.3 & 0.3 & 3.5 & & 4 & & $<0.01$ \\
\hline$\overline{1246}$ & 30 & 20 & 0.3 & 0.08 & 0.3 & 0.3 & 0.1 & & 47 & & $<0.01$ \\
\hline 1247 & 43 & 23 & 2.7 & 0.08 & 0.7 & 0.06 & 0.4 & & 27 & & $<0.01$ \\
\hline
\end{tabular}

Values in parentheses are not certifed, but are given for information only. 


\section{Tool Steels}

\begin{tabular}{|c|c|c|c|c|c|c|c|c|c|c|c|}
\hline & \multirow{2}{*}{\multicolumn{2}{|c|}{ SRM }} & \multirow[b]{2}{*}{ Type } & \multicolumn{8}{|c|}{ Chemical Composition (Nominal Weight Percent) } \\
\hline & & & & Mn & $\mathrm{Si}$ & $\mathrm{Cu}$ & $\mathrm{Cr}$ & V & Mo & $\mathbf{W}$ & Co \\
\hline 436 & & & Special (Cr6-Mo3-W 10) & 0.21 & 0.32 & 0.075 & 6.02 & 0.63 & 2.80 & 9.7 & \\
\hline 437 & 837 & D837 & Special (Cr8-Mo2-W3-Co3) & 0.48 & 0.53 & & 7.79 & 3.04 & 1.50 & 2.8 & 2.9 \\
\hline 438 & & & Mo High Speed (AISI-SAE-M30) & 0.20 & 0.17 & 0.17 & 4.66 & 1.17 & 8.26 & 1.7 & 4.9 \\
\hline 439 & & & Mo High Speed (AISI-SAE-M36) & 0.18 & 0.21 & 0.12 & 2.72 & 1.50 & 4.61 & 5.7 & 7.8 \\
\hline 440 & 840 & $\mathrm{D} 840$ & Special W High Speed (Cr2-W13-Co12) & 0.15 & 0.14 & 0.059 & 2.12 & 2.11 & 0.070 & 13.0 & 11.8 \\
\hline 441 & & $\mathrm{D} 841$ & W High Speed (AISI-SAE-T1) & 0.27 & 0.16 & 0.072 & 4.20 & 1.13 & 0.84 & 18.5 & \\
\hline
\end{tabular}

\begin{tabular}{|c|c|c|c|c|c|c|c|c|c|c|c|c|c|}
\hline SRM & Type & $\mathrm{C}$ & Mn & $\mathbf{P}$ & $\mathrm{S}$ & $\mathrm{Si}$ & $\mathrm{Cu}$ & $\mathrm{Ni}$ & $\mathrm{Cr}$ & V & Mo & $\mathbf{W}$ & $\mathrm{Co}$ \\
\hline 1157 & Tool (AISI M2) & 0.836 & 0.34 & 0.011 & 0.004 & 0.18 & 0.088 & 0.228 & 4.36 & 1.82 & 4.86 & 6.28 & 0.028 \\
\hline
\end{tabular}

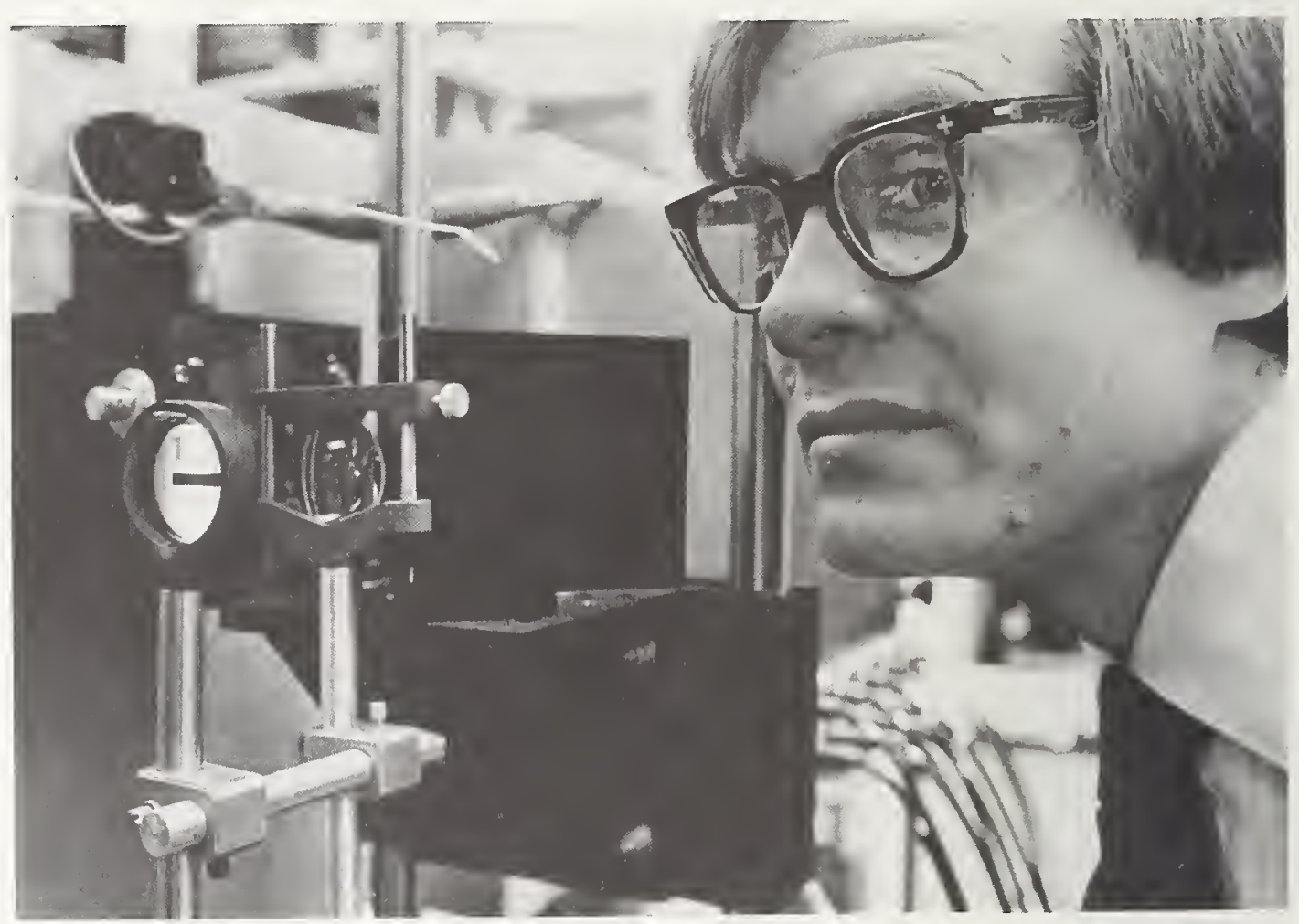

Robert Watters checks the atignment of the plasma flame image on the entrance mask of the inductively coupled plasma (ICP)

spectrometer. A five-millimeter aperature is spectrometer. A five-millimeter aperature is
centered at the observation height of the centered at the observation height of the
plasma. This height is chosen to assure the plasma. This height is chosen to assure the
best sensitivity for simultaneous inultielement analysis using the $I C P$.

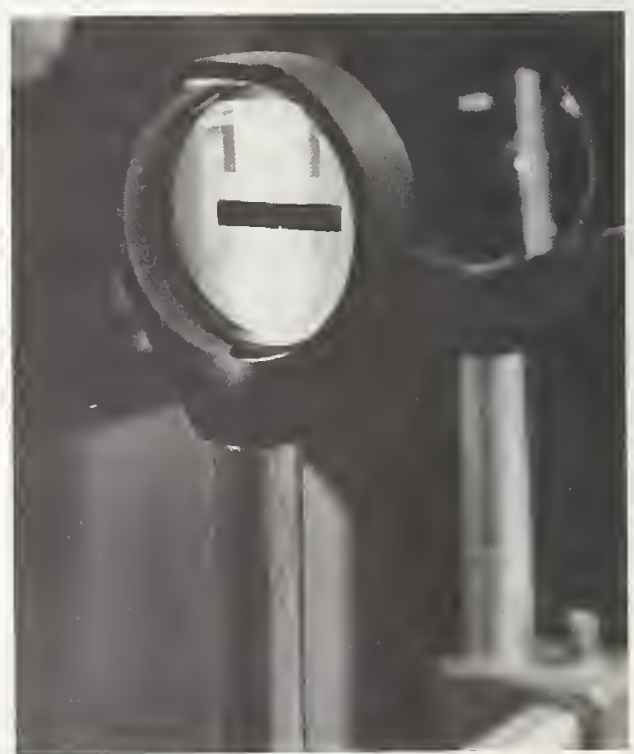




\section{Steelmaking Alloys}

These SRM's provide standards of known chemical composition primarily for checking chemical methods of analysis for the major constituents and for selected minor elements covered by ASTM specifications. They are furnished as fine powders (usually $<0.1 \mathrm{~mm}$ ). These SRM's are finding increased use in calibration with instrumental methods of analysis.

\begin{tabular}{|c|c|c|c|c|c|c|c|c|c|c|}
\hline \multirow[b]{2}{*}{ SRM } & \multirow[b]{2}{*}{ Type } & \multirow[b]{2}{*}{$\begin{array}{l}\text { Wt/Unit } \\
\text { (grams) }\end{array}$} & \multicolumn{8}{|c|}{ Chemical Composition (Nominal Weight Percent) } \\
\hline & & & $\mathbf{C}$ & Mn & $\mathbf{P}$ & $\mathrm{S}$ & $\mathrm{Si}$ & $\mathrm{Cu}$ & $\mathrm{Ni}$ & $\mathbf{O}$ \\
\hline $57 \mathrm{a}$ & Refined Silicon & 60 & 0.024 & 0.015 & 0.003 & 0.003 & 98.55 & 0.004 & 0.008 & $(-0.3)$ \\
\hline $58 \mathrm{a}$ & Ferrosilicon $(73 \% \mathrm{Si})$ & 75 & 0.014 & 0.16 & 0.009 & $<0.002$ & 73.20 & 0.024 & 0.012 & $(0.20)$ \\
\hline $59 a$ & Ferrosilicon (50\% Si) & 50 & 0.046 & 0.75 & 0.016 & 0.002 & 48.10 & 0.052 & 0.033 & \\
\hline 195 & $\begin{array}{l}\text { Ferrosilicon }(75 \% \mathrm{Si}) \\
\text { Hi-Purity }\end{array}$ & 75 & 0.034 & 0.17 & 0.02 & $<0.002$ & 75.3 & 0.047 & 0.032 & 0.42 \\
\hline $64 c$ & Ferrochromium (HC) & 100 & 4.68 & 0.16 & 0.020 & 0.067 & 1.22 & 0.005 & 0.43 & \\
\hline 196 & Ferrochromium (LC) & 100 & 0.035 & $(0.282)$ & 0.020 & 0.003 & 0.373 & & & \\
\hline 71 & Calcium Molybdate & 60 & & & & & & & & \\
\hline 90 & Ferrophosphorus & 75 & & & 26.2 & & & & & \\
\hline 340 & Ferroniobium & 100 & 0.061 & 1.70 & 0.036 & & 4.39 & & 0.063 & \\
\hline $68 c$ & Ferromanganese (HC) & 100 & 6.72 & 80.04 & 0.19 & 0.008 & 0.225 & & & \\
\hline 689 & Ferrochromium Silicon & 100 & 0.043 & 0.32 & 0.026 & 0.002 & 39.5 & 0.013 & 0.20 & $(0.06)$ \\
\hline
\end{tabular}

\begin{tabular}{|c|c|c|c|c|c|c|c|c|c|c|c|}
\hline SRM & $\mathrm{Cr}$ & V & Mo & $\mathrm{Ti}$ & Al & $\mathrm{Nb}$ & $\mathrm{Zr}$ & $\mathrm{Ca}$ & $\mathrm{Fe}$ & B & As \\
\hline $57 \mathrm{a}$ & 0.024 & 0.013 & $\mathrm{~Pb}<0.001$ & 0.040 & 0.47 & & 0.002 & 0.17 & 0.50 & 0.001 & $<0.001$ \\
\hline $58 \mathrm{a}$ & 0.020 & $(0.002)$ & $(0.01)$ & 0.051 & 0.95 & $\mathrm{Co}<0.01$ & 0.002 & 0.30 & 25.23 & 0.0010 & \\
\hline $59 a$ & 0.08 & & & & 0.35 & & & 0.042 & 50.05 & 0.058 & \\
\hline 195 & 0.047 & $(0.001)$ & $(0.01)$ & 0.037 & 0.046 & $\mathrm{Co}<0.01$ & $(<0.02)$ & 0.053 & 23.6 & 0.001 & $(0.0024)$ \\
\hline $64 c$ & 68.00 & 0.15 & & 0.02 & & $\mathrm{Co} 0.051$ & & N0.045 & 24.98 & & \\
\hline$\overline{196}$ & 70.83 & $(0.12)$ & & & & & & & & & \\
\hline 71 & & & 35.29 & 0.063 & & & & & 1.92 & & \\
\hline \multicolumn{12}{|l|}{90} \\
\hline 340 & & & & 0.89 & & 57.51 & Ta3.73 & & & & \\
\hline $68 \mathrm{c}$ & 0.074 & & & & & & & & 12.3 & & 0.021 \\
\hline 689 & 36.4 & 0.09 & $\mathrm{~Pb}(0.004)$ & 0.40 & 0.049 & $\mathrm{Co} 0.034$ & $\mathrm{Bi}(<0.003)$ & $\mathrm{N}(0.002)$ & 23.2 & 0.0017 & $(0.009)$ \\
\hline
\end{tabular}

Values in parentheses are not certified, but are given for information only. 


\section{Cast Irons (Chip Form)}

This group of cast iron SRM's is similar to the chip-form steels and was prepared for use in checking chemical methods in the cast iron industry. These SRM's are furnished in 150-g units (unless otherwise noted) in the form of chips usually sized between 0.7 to $1.2 \mathrm{~mm}$. They are prepared by lathe cutting of chips with a multiple-tooth cutting tool from thin-wall cylindrical castings especially made for this purpose. SRM's 890 , 891 , and 892 are provided in the form of granules/powders. Supplied with each SRM is a Certificate of Analysis listing the chemical composition determined at NBS and other laboratories that cooperated in the certification of the SRM's. For SRM 365, Electrolytic Iron, the Certificate provides information on these additional elements: W, Nb, Ag, Zn, Ge, O, H, Ta, Nd, Zr, Sb, Bi, Ca, Mg, Se, Te, Ce, La, Pr, Au, Hf, and $\mathrm{Fe}$.

\begin{tabular}{|c|c|c|c|c|c|c|c|c|c|}
\hline \multirow{3}{*}{ SRM } & \multirow{3}{*}{ Type } & \multicolumn{8}{|c|}{ Chemical Composition (Nominal Weight Percent) } \\
\hline & & \multicolumn{2}{|c|}{$\mathrm{C}$} & \multirow[t]{2}{*}{$\mathbf{M n}$} & \multirow[t]{2}{*}{$\mathbf{P}$} & \multicolumn{2}{|c|}{$\mathbf{S}$} & \multirow[t]{2}{*}{$\mathrm{Si}$} & \multirow[t]{2}{*}{$\mathrm{Cu}$} \\
\hline & & Total & Graphitic & & & Grav & Comb & & \\
\hline $3 \mathrm{~d}$ & White $(110 \mathrm{~g})$ & 2.54 & & 0.40 & 0.025 & & 0.052 & 1.31 & 0.043 \\
\hline $4 \mathrm{k}$ & Cast & $3.2_{2}$ & $2.6_{5}$ & $0.82_{5}$ & 0.149 & & 0.043 & 1.33 & $0.24_{3}$ \\
\hline $5 \mathrm{~L}$ & Cast & 2.60 & 1.98 & 0.68 & 0.2804 & & 0.124 & 1.82 & 1.01 \\
\hline $6 \mathrm{~g}$ & Cast & 2.85 & 2.01 & 1.05 & 0.557 & & 0.124 & 1.05 & 0.502 \\
\hline $7 \mathrm{~g}$ & Cast (High Phosphorus) & 2.69 & 2.59 & 0.612 & 0.794 & 0.061 & 0.060 & 2.41 & 0.128 \\
\hline $82 b$ & Cast (Ni-Cr) & 2.85 & 2.37 & 0.745 & 0.025 & & 0.007 & 2.10 & 0.038 \\
\hline $107 \mathrm{c}$ & Cast (Ni-Cr-Mo) & 2.99 & 1.98 & 0.480 & 0.079 & & 0.059 & 1.21 & 0.205 \\
\hline $115 \mathrm{a}$ & Cast (Cu-Ni-Cr) & 2.62 & 1.96 & 1.00 & 0.086 & 0.064 & 0.065 & 2.13 & 5.52 \\
\hline $122 \mathrm{~h}$ & Cast (Car Wheel) & 3.52 & 2.82 & 0.543 & 0.311 & & 0.072 & 0.513 & 0.028 \\
\hline 334 & Gray Cast & 2.83 & $(2.3)$ & $(0.82)$ & $(0.14)$ & & 0.043 & $(1.3)$ & $(0.24)$ \\
\hline 338 & White Cast & 3.33 & & $(0.76)$ & $(0.054)$ & & 0.015 & $(1.82)$ & $(0.27)$ \\
\hline 341 & Ductile & 1.81 & 1.23 & 0.92 & 0.024 & 0.007 & 0.007 & 2.44 & 0.152 \\
\hline $342 a$ & Nodular & 1.86 & 1.38 & 0.274 & 0.019 & & 0.006 & 2.73 & 0.135 \\
\hline 365 & Electrolytic Iron & 0.0068 & & 0.0056 & 0.0025 & & 0.0055 & 0.0080 & 0.0058 \\
\hline 890 & $\begin{array}{l}\text { High Alloy White Cast } \\
(\mathrm{HC} 0259+\mathrm{V})\end{array}$ & 2.91 & & 0.62 & 0.025 & & 0.015 & 0.67 & 0.055 \\
\hline 891 & $\begin{array}{c}\text { High Alloy White Cast } \\
\text { (Ni-Hard, Type I) }\end{array}$ & 2.71 & & 0.55 & 0.038 & & 0.029 & 0.56 & 0.150 \\
\hline 892 & $\begin{array}{l}\text { High Alloy White Cast } \\
\text { (Ni-Hard, Type IV) }\end{array}$ & 3.33 & & 0.76 & 0.054 & & 0.015 & 1.83 & 0.270 \\
\hline
\end{tabular}




\section{(Continued)}

\begin{tabular}{|c|c|c|c|c|c|c|}
\hline SRM & $\mathrm{Ni}$ & $\mathrm{Cr}$ & V & Mo & Co & $\mathrm{Ti}$ \\
\hline $3 d$ & 0.025 & 0.03 & $(0.002)$ & $(0.007)$ & & $(0.003)$ \\
\hline $4 \mathrm{k}$ & 0.042 & 0.116 & 0.024 & 0.040 & $\mathrm{Zn}(<0.001)$ & $(0.03)$ \\
\hline $5 \mathrm{~L}$ & 0.086 & 0.148 & 0.034 & 0.020 & & 0.050 \\
\hline $6 \mathrm{~g}$ & 0.135 & 0.370 & 0.056 & 0.035 & & 0.059 \\
\hline $7 \mathrm{~g}$ & 0.120 & 0.048 & 0.010 & 0.012 & & 0.044 \\
\hline$\overline{82 b}$ & 1.22 & 0.333 & 0.027 & 0.002 & & 0.027 \\
\hline $107 \mathrm{c}$ & 2.20 & 0.693 & 0.015 & 0.83 & & 0.19 \\
\hline $115 a$ & 14.49 & 1.98 & 0.014 & 0.050 & & 0.020 \\
\hline $122 \mathrm{~h}$ & 0.078 & 0.052 & 0.041 & $(0.003)$ & & 0.034 \\
\hline 334 & $(0.04)$ & $(0.12)$ & $(0.025)$ & $(0.040)$ & & $(0.03)$ \\
\hline 338 & $(5.5)$ & $(10.2)$ & $(0.04)$ & & $(0.32)$ & \\
\hline 341 & 20.32 & 1.98 & 0.012 & 0.010 & & 0.018 \\
\hline$\overline{342 a}$ & 0.06 & 0.058 & & 0.006 & & 0.020 \\
\hline 365 & 0.041 & $0.007_{2}$ & 0.0006 & 0.0050 & $0.007_{2}$ & 0.0006 \\
\hline 890 & 0.397 & 32.4 & 0.45 & 0.018 & $(0.03)$ & \\
\hline 891 & 4.48 & 2.23 & 0.039 & 0.27 & 0.19 & $(0.01)$ \\
\hline 892 & 5.53 & 10.18 & 0.041 & 0.20 & 0.31 & $(0.02)$ \\
\hline
\end{tabular}

\begin{tabular}{|c|c|c|c|c|c|c|}
\hline SRM & As & Sn & $\underset{\text { (total) }}{\mathrm{Al}}$ & $\mathrm{Mg}$ & $\mathbf{N}$ & $\mathrm{Fe}$ \\
\hline \multicolumn{7}{|l|}{$3 d$} \\
\hline $4 \mathrm{k}$ & $(0.03)$ & $(0.004)$ & $(0.004)$ & $\mathrm{Sb}(<0.001)$ & $(0.0016)$ & $\mathrm{Pb}(0.001)$ \\
\hline $5 \mathrm{~L}$ & & & & & 0.005 & \\
\hline $6 \mathrm{~g}$ & 0.042 & & & & 0.005 & \\
\hline $7 \mathrm{~g}$ & 0.014 & & & & 0.004 & \\
\hline \multicolumn{7}{|l|}{$82 \mathrm{~b}$} \\
\hline \multicolumn{7}{|l|}{$107 c$} \\
\hline \multicolumn{7}{|l|}{$115 a$} \\
\hline \multicolumn{7}{|l|}{$122 \mathrm{~h}$} \\
\hline 334 & $(0.03)$ & $(0.004)$ & $(0.004)$ & & $(0.0017)$ & \\
\hline \multicolumn{7}{|l|}{338} \\
\hline 341 & & & & 0.068 & & \\
\hline $342 a$ & & & & 0.070 & & \\
\hline 365 & $(0.0002)$ & $-(0.0002)$ & $(0.0007)$ & Pb0.000019 & 0.001 & 99.90 \\
\hline 890 & $(0.008)$ & & $(<0.01)$ & & $(0.089)$ & $(61.8)$ \\
\hline 891 & $(0.004)$ & $(<0.01)$ & $(0.008)$ & & $(0.012)$ & $(88.5)$ \\
\hline$\overline{892}$ & $(0.006)$ & $(0.02)$ & $(0.009)$ & & $(0.019)$ & $(77.4)$ \\
\hline
\end{tabular}

Values in parentheses are not certified, but are for information only. 


\section{Cast Steels, White Cast Irons, Ductile Irons and Blast Furnace Irons (Solid Form)}

These chill-cast SRM's were prepared for use in analytical control of cast steels and cast irons by rapid instrumental methods. Although employed in x-ray spectroscopic analysis, they are particularly useful for calibrating vacuum optical emission spectrometers because they permit the determination of carbon, phosphorus, and sulfur in addition to the metallic elements.

The "benchmark" concept was used in preparing three new white irons $(1145,1146$, and 1150) with compositions tailored to provide low, nominal, and high values for elements normally specified in cast iron materials, as well as most malleable, ductile, and grey irons. A concentration range for a number of trace elements of interest was provided to enhance the utility of the standards. The planning, preparation, homogeneity testing, and analysis of these SRM's were done through a cooperative Industry-ASTM-NBS program.

These SRM's are chill-cast sections. Details of the preparation and intended use of the SRM's are given in NBS Miscellaneous Publication 260-1.

\begin{tabular}{|c|c|c|c|c|c|c|c|c|c|c|}
\hline \multirow[b]{2}{*}{ SRM } & \multirow{2}{*}{\multicolumn{2}{|c|}{ Type }} & \multicolumn{8}{|c|}{ Chemical Composition (Nominal Weight Percent) } \\
\hline & & & C & Mn & $\mathbf{P}$ & $\mathbf{S}$ & Si & $\mathrm{Cu}$ & $\mathrm{Ni}$ & $\mathrm{Cr}$ \\
\hline $1138 \mathrm{a}$ & \multicolumn{2}{|c|}{ Cast Steel (No. 1) } & $0.11_{8}$ & 0.35 & 0.035 & 0.056 & 0.25 & 0.09 & 0.10 & 0.13 \\
\hline $1139 a$ & \multicolumn{2}{|c|}{ Cast Steel (No. 2) } & $0.79_{0}$ & 0.92 & 0.012 & 0.013 & 0.80 & 0.47 & 0.98 & $2.1_{8}$ \\
\hline $1143 a$ & \multicolumn{2}{|c|}{ Blast Furnace (1) } & 4.08 & $0.29_{6}$ & $0.16_{4}$ & 0.067 & 1.60 & $0.13_{8}$ & $0.11_{6}$ & $0.16_{3}$ \\
\hline $1144 \mathrm{a}$ & \multicolumn{2}{|c|}{ Blast Furnace (2) } & 4.32 & 1.23 & $0.08_{4}$ & 0.083 & $0.18_{2}$ & $0.09_{1}$ & $0.06_{3}$ & 0.029 \\
\hline 1145 & \multicolumn{2}{|c|}{ White Cast Iron } & 2.85 & 0.040 & 0.24 & 0.21 & 0.29 & 0.52 & 0.59 & 0.67 \\
\hline 1146 & \multicolumn{2}{|c|}{ White Cast Iron } & 2.01 & 1.64 & 0.55 & 0.022 & 3.68 & 1.49 & 3.01 & 2.56 \\
\hline 1150 & \multicolumn{2}{|c|}{ White Cast Iron } & 3.48 & 0.81 & 0.063 & 0.070 & 1.24 & 0.092 & 0.074 & 0.95 \\
\hline $\mathrm{C} 1173$ & \multicolumn{2}{|c|}{ Cast Steel 3} & 0.453 & 0.174 & 0.031 & 0.092 & 1.38 & 0.204 & 4.04 & 2.63 \\
\hline$\overline{1173}$ & \multicolumn{2}{|c|}{ Ni-Cr-Mo-V Steel } & 0.423 & 0.19 & 0.033 & 0.092 & 1.28 & 0.204 & 4.06 & 2.70 \\
\hline SRM & $\mathbf{V}$ & Mo & \multicolumn{2}{|c|}{ Ti } & \multicolumn{2}{|l|}{ As } & Al & & $\mathrm{Te}$ & Co \\
\hline $1138 \mathrm{a}$ & 0.020 & 0.05 & \multicolumn{2}{|c|}{$(0.0012)$} & \multicolumn{2}{|l|}{$(<0.005)$} & $(0.067)$ & & & \\
\hline $1139 a$ & 0.26 & 0.51 & \multicolumn{2}{|c|}{$(0.004)$} & \multicolumn{2}{|l|}{$(<0.005)$} & $(0.13)$ & & & \\
\hline $1143 \mathrm{a}$ & 0.018 & $(0.004)$ & \multicolumn{2}{|c|}{$0.08_{7}$} & \multicolumn{2}{|l|}{$(0.003)$} & $(0.008)$ & \multicolumn{2}{|r|}{$0.01_{6}$} & \\
\hline $1144 \mathrm{a}$ & $0.02_{5}$ & $(0.007)$ & \multicolumn{2}{|c|}{0.32} & \multicolumn{2}{|l|}{$(0.004)$} & $(<0.005)$ & \multicolumn{2}{|r|}{$0.02_{2}$} & \\
\hline 1145 & 0.11 & 0.48 & \multicolumn{2}{|c|}{0.017} & & & & & & 0.058 \\
\hline 1146 & 0.20 & 1.51 & \multicolumn{2}{|c|}{0.20} & & & & & & 0.13 \\
\hline 1150 & 0.034 & 0.074 & \multicolumn{2}{|c|}{0.045} & & & & & & 0.014 \\
\hline $\mathrm{C} 1173$ & 0.42 & 1.46 & \multicolumn{2}{|c|}{0.037} & \multicolumn{2}{|l|}{$(0.02)$} & $(0.005)$ & & & 0.064 \\
\hline 1173 & 0.42 & 1.50 & & 15) & & & & \multicolumn{2}{|c|}{$\mathrm{Nb}(0.045)$} & 0.076 \\
\hline
\end{tabular}

Values in parentheses are not certified, but are given for information only. 
These SRM's provide materials of known composition for checking the performance of chemical methods of analysis and in calibration with instrumental methods. The aluminum-, magnesium-, and zinc-base alloys are furnished as approximately 0.4 to $1.4 \mathrm{~mm}$ chips prepared by cutting thin wall castings or wrought bar stock. Certificates of Analysis provided with these standards give the composition as determined at NBS, and most give values obtained by industrial and other outside laboratories cooperating in certification of the standards.

\section{Aluminum-Base Alloys}

\begin{tabular}{|c|c|c|c|c|c|c|c|c|c|c|c|}
\hline \multirow[b]{2}{*}{ SRM } & \multirow{2}{*}{\multicolumn{3}{|c|}{ Type }} & & \multirow[b]{2}{*}{$\begin{array}{l}\text { Wt/Unit } \\
\text { (grams) }\end{array}$} & \multicolumn{6}{|c|}{ Chemical Composition (Nominal Weight Percent) } \\
\hline & & & & & & $\mathrm{Mn}$ & $\mathrm{Si}$ & $\mathrm{Cu}$ & $\mathrm{Ni}$ & $\mathrm{Cr}$ & V \\
\hline $85 b \quad V$ & Wrought ( & & & & 75 & 0.61 & 0.18 & 3.99 & 0.084 & 0.211 & 0.006 \\
\hline $87 \mathrm{a} \quad A$ & Al-Si (Chip & & & & 75 & 0.26 & 6.24 & 0.30 & 0.57 & 0.11 & $\langle 0.01$ \\
\hline $855 \mathrm{C}$ & Casting All & 56 (fine $\mathrm{mi}$ & nillings) & & 30 & 0.057 & 7.17 & 0.13 & 0.015 & 0.013 & \\
\hline$\overline{856 \quad \mathrm{C}}$ & Casting All & 30 (fine $\mathrm{mi}$ & nillings) & & 30 & 0.35 & 9.21 & 3.51 & 0.37 & 0.055 & \\
\hline $858 \quad A$ & Alloy 6011 & dified ) ( & (fine millings) & & 35 & 0.48 & 0.79 & 0.84 & 0.0006 & 0.0011 & 0.0030 \\
\hline$\overline{859 \quad A}$ & Alloy 7075 & millings) & & & 35 & 0.078 & 0.17 & 1.59 & 0.063 & 0.176 & 0.0082 \\
\hline SRM & $\mathrm{Ti}$ & Sn & Ga & $\mathrm{Fe}$ & & $\mathrm{Pb}$ & & $\mathrm{Mg}$ & $\mathrm{Zn}$ & & $\mathrm{Be}$ \\
\hline $85 b$ & 0.022 & & 0.019 & 0.24 & & 0.021 & & 49 & 0.03 & & \\
\hline $87 \mathrm{a}$ & 0.18 & 0.05 & 0.02 & 0.61 & & 0.10 & & 37 & 0.16 & & \\
\hline 855 & 0.15 & 0.010 & & 0.16 & & 0.015 & & 37 & 0.08 & & \\
\hline 856 & 0.068 & 0.10 & & 0.92 & & 0.10 & & 061 & 0.96 & & \\
\hline 858 & 0.042 & & & 0.078 & & & & 01 & 1.04 & & $<0.0001$ \\
\hline 859 & 0.041 & & & & & & & 45 & 5.46 & & 0.0026 \\
\hline
\end{tabular}

\section{Copper-Base Alloys}

SRM's $871,872,874,875,879$, and 880 are fine granules produced by a water atomization technique for use primarily in checking chemical methods of analysis. The homogeneity of these materials is exceptionally high, and for certain alloys such as SRM 872, Phosphor Bronze (CDA 544), it is the only form that can readily be prepared to exhibit acceptable homogeneity. For many alloys (for which homogeneity requirements can be met) both granules (or chips) for chemical analysis and solids for optical emission and $\mathrm{x}$-ray fluorescence methods, are desirable.

\begin{tabular}{|c|c|c|c|c|c|c|c|}
\hline \multirow[b]{2}{*}{ SRM } & \multirow[b]{2}{*}{ Type } & \multirow{2}{*}{$\begin{array}{l}\text { Wt/Unit } \\
\text { (grams) }\end{array}$} & \multicolumn{5}{|c|}{ Chemical Composition (Nominal Weight Percent) } \\
\hline & & & $\mathrm{Cu}$ & $\mathbf{N i}$ & $\mathrm{Fe}$ & $\mathrm{Zn}$ & $\mathrm{Pb}$ \\
\hline $37 \mathrm{e}$ & Brass, Sheet & 150 & 69.61 & 0.53 & 0.004 & 27.85 & 1.00 \\
\hline$\overline{158 \mathrm{a}}$ & Bronze, Silicon & 150 & 90.93 & 0.001 & 1.23 & 2.08 & 0.097 \\
\hline 871 & Bronze, Phosphor (CDA 521) & 100 & 91.68 & & $<0.001$ & 0.025 & 0.010 \\
\hline 872 & Bronze, Phosphor (CDA 544) & 100 & 87.36 & & 0.003 & 4.0 & 4.13 \\
\hline 874 & Cupro-Nickel, 10\% (CDA 706)"High-Purity" & 100 & 88.49 & 10.18 & 1.22 & 0.002 & $<0.0005$ \\
\hline 875 & Cupro-Nickel, 10\% (CDA 706)"Doped" & 100 & 87.83 & 10.42 & 1.45 & 0.11 & 0.0092 \\
\hline 879 & Nickel Silver (CDA 762) & 100 & 57.75 & 12.11 & 0.0020 & 30.04 & 0.002 \\
\hline 880 & Nickel Silver (CDA 770) & 100 & 54.51 & 18.13 & 0.004 & 27.3 & 0.002 \\
\hline 1034 & *Unalloyed Copper & rod & $(99.96)$ & $(0.6)$ & $(2.0)$ & $(<11)$ & $(0.5)$ \\
\hline 1035 & **Leaded-Tin Bronze Alloy & 50 & $(78.5)$ & $(0.75)$ & $(0.001)$ & $(0.25)$ & $(13.5)$ \\
\hline
\end{tabular}




\section{Copper Base Alloys (Continued)}

\begin{tabular}{|c|c|c|c|c|c|c|c|c|c|c|c|}
\hline SRM & Mn & Sb & Sn & $\mathrm{Cr}$ & $\mathbf{P}$ & $\mathrm{Ag}$ & $\mathrm{Si}$ & Al & $\mathrm{Te}$ & $\mathrm{Cd}$ & Se \\
\hline $37 \mathrm{e}$ & & & 1.00 & & & & & & & & \\
\hline $158 \mathrm{a}$ & 1.11 & & 0.96 & & 0.026 & & 3.03 & 0.46 & & & \\
\hline 871 & & & 8.14 & & 0.082 & & & & & & \\
\hline 872 & & & 4.16 & & 0.26 & & & & & & \\
\hline 874 & 0.0020 & $<0.001$ & 0.007 & & 0.002 & & $(0.0006)$ & & & $<0.0002$ & 0.00015 \\
\hline 875 & $\langle 0.0007$ & $<0.001$ & 0.009 & & 0.0020 & & $(0.0008)$ & & & 0.0022 & 0.0004 \\
\hline 879 & $<0.001$ & & & & & & & & & & \\
\hline 880 & $<0.001$ & & & & & & & & & & \\
\hline 1034 & $(<0.1)$ & $(0.2)$ & $(<0.2)$ & $(0.3)$ & & $(8.1)$ & $(<2)$ & $(<2)$ & $(0.5)$ & $(<1)$ & (3.3) \\
\hline 1035 & & & $(6.8)$ & & & & & & & & \\
\hline
\end{tabular}

\begin{tabular}{|c|c|c|c|c|c|c|c|c|c|c|}
\hline SRM & $\mathrm{Bi}$ & O & Co & C & Au & H & $S$ & As & $\mathrm{Mg}$ & $\mathrm{Ti}$ \\
\hline \multicolumn{11}{|l|}{$37 \mathrm{e}$} \\
\hline \multicolumn{11}{|l|}{$158 \mathrm{a}$} \\
\hline \multicolumn{11}{|l|}{871} \\
\hline \multicolumn{11}{|l|}{872} \\
\hline 874 & $<0.0002$ & $(0.06)$ & & $(0.0023)$ & & $(0.0016)$ & $(0.0011)$ & $(<0.0006)$ & $(0.0002)$ & $(0.0001)$ \\
\hline 875 & 0.003 & $(0.14)$ & & $(0.0035)$ & & $(0.004)$ & $(0.0011)$ & $(0.0010)$ & $(0.0010)$ & $(<0.0002)$ \\
\hline \multicolumn{11}{|l|}{879} \\
\hline \multicolumn{11}{|l|}{880} \\
\hline 1034 & $(0.2)$ & $(363)$ & $(0.02)$ & & $(<0.05)$ & & 2.8 & $(0.2)$ & $(<1)$ & \\
\hline 1035 & & 0.64 & & & & & 22.3 & & P 0.004 & \\
\hline
\end{tabular}

Values in parentheses are not certified, but are given for information only.

*Values for SRM 1034 are ppm by weight.

**Sulfur value for SRM 1035 is ppm by weight.

One casting provides a grid of seventy-two rough disks for SRM C1146a, White Cast Iron

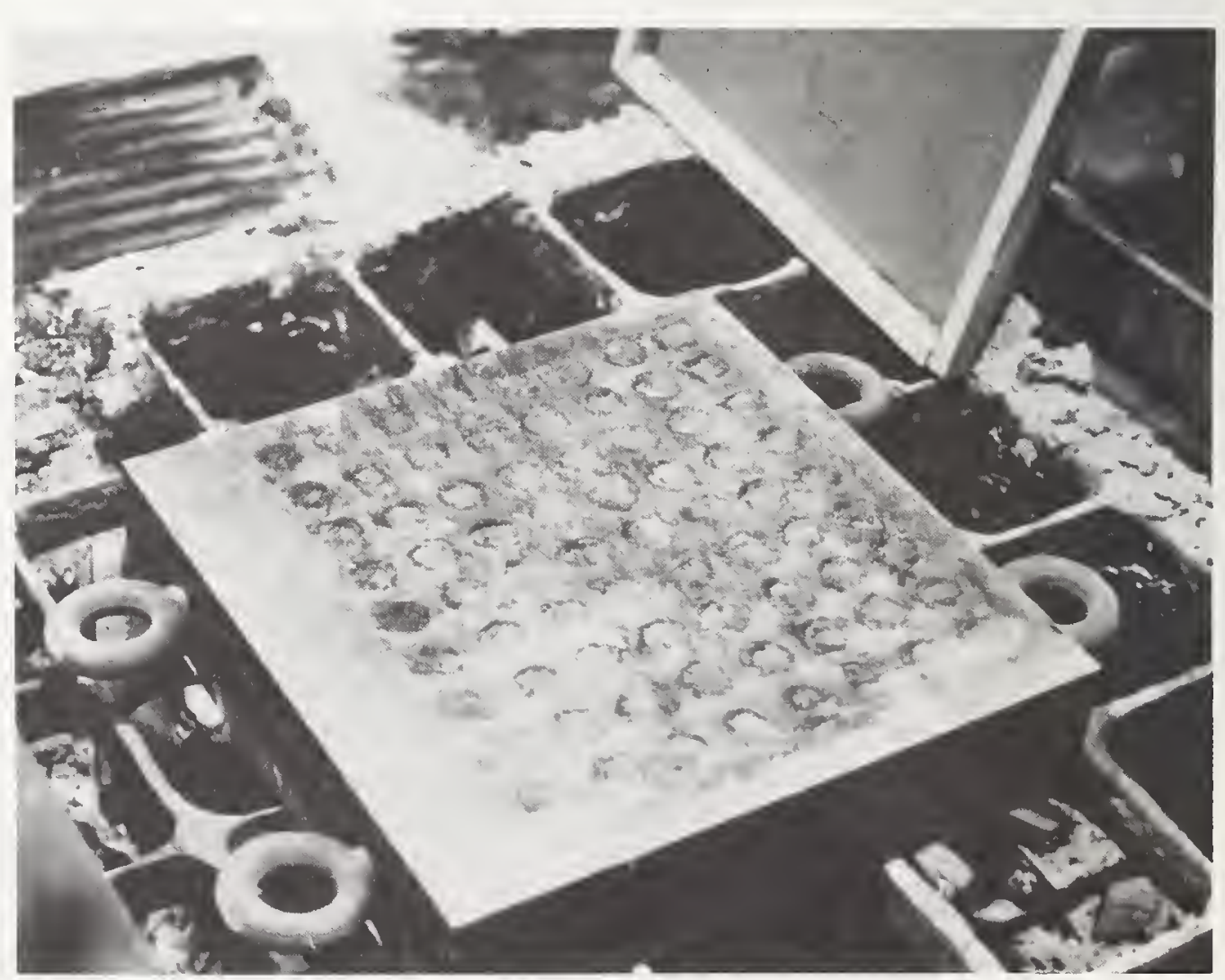




\section{Copper "Benchmark"}

The copper "benchmark" standards were prepared in a cooperative Industry-ASTM-NBS program and were designed primarily for use in calibration with optical emission methods of analysis. They should also serve in the development of other methods of trace analysis. Twelve different compositions are issued as 25 SRM's. $\mathrm{Cu}$ " 0 " and $\mathrm{Cu}$ XI are issued in chip form only. Cu IV is available only in rod form $6.6 \mathrm{~mm}$ in diameter and $103 \mathrm{~mm}$ long. Cu I, II, III, V, VI, and VII are available both as chips and as rods $6.35 \mathrm{~mm}$ in diameter and $103 \mathrm{~mm}$ long. $\mathrm{Cu}$ VIII, IX, and $\mathrm{X}$ are issued as chill-cast and unidirectionally solidified blocks $32 \mathrm{~mm}$ square and $19 \mathrm{~mm}$ thick. Cu VIII-Cu X are phosphorized copper containing a nominal concentration range from about 10 to $500 \mathrm{ppm}$ for the same 20 trace elements contained in the other copper "benchmark" SRM's, plus five to eight additional elements. These SRM's are also applicable for x-ray fluorescence methods of analysis and, because of deliberate additions of gold and silver (in ratios of 1 to 4), for calibration of fire assay equipment.

\begin{tabular}{|c|c|c|c|c|c|c|c|c|c|c|c|c|}
\hline \multirow[b]{2}{*}{ SRM } & \multirow{2}{*}{\multicolumn{2}{|c|}{ Type }} & & \multirow[b]{2}{*}{$\begin{array}{l}\text { Wt/Unit } \\
\text { (grams) }\end{array}$} & \multicolumn{8}{|c|}{ Chemical Composition (Nominal Parts Per Million by Weight) } \\
\hline & & & & & $\mathbf{S b}$ & As & $\mathrm{Bi}$ & $\mathrm{Cr}$ & Co & $\mathrm{Fe}$ & $\mathrm{Pb}$ & Mn \\
\hline 393 & \multicolumn{3}{|c|}{ Unalloyed-Cu " 0 " } & 50 & 0.25 & 0.41 & $<0.1$ & $<0.5$ & 0.02 & $<1$ & 0.039 & $\langle 0.01$ \\
\hline 394 & \multicolumn{3}{|c|}{ Unalloyed-Cu I } & 50 & 4.5 & 2.6 & 0.35 & 2.0 & 0.5 & 147 & 26.5 & 3.7 \\
\hline 395 & \multicolumn{3}{|c|}{ Unalloyed-Cu II } & 50 & 8.0 & 1.6 & 0.50 & 6.0 & 0.3 & 96 & 3.25 & 5.3 \\
\hline 396 & \multicolumn{3}{|c|}{ Unalloyed-Cu III } & 50 & $<1$ & $\langle 0.2$ & 0.07 & 4.3 & 0.4 & 143 & 0.41 & 7.5 \\
\hline 398 & \multicolumn{3}{|c|}{ Unalloyed-Cu V } & 50 & 7.5 & 25 & 2.0 & $(0.3)$ & 2.8 & 11.4 & 9.9 & $(0.3)$ \\
\hline 399 & \multicolumn{3}{|c|}{ Unalloyed-Cu VI } & 50 & 30 & 47 & 10.5 & $(0.5)$ & 0.5 & 20.0 & 114 & $(0.3)$ \\
\hline 400 & \multicolumn{3}{|c|}{ Unalloyed-Cu VII } & 50 & 102 & 140 & 24.5 & $(0.5)$ & 0.6 & 41 & 128 & $(0.2)$ \\
\hline 454 & \multicolumn{3}{|c|}{ Unalloyed_Cu XI } & 35 & 24 & 46 & \multicolumn{2}{|l|}{19} & (4) & $(50)$ & \multicolumn{2}{|l|}{66} \\
\hline SRM & $\mathrm{Cu}(\mathrm{Wt} \%)$ & $\mathrm{Ni}$ & Se & Ag & $\mathbf{S}$ & Te & Sn & Zn & Al & $\mathrm{Cd}$ & Au & $\mathbf{M g}$ \\
\hline 393 & 99.998 & 0.05 & $<0.05$ & 0.10 & $<1$ & $<0.5$ & $<0.1$ & $<0.1$ & $<0.1$ & $<0.1$ & $<0.05$ & $<0.1$ \\
\hline 394 & 99.908 & 11.7 & 2.1 & 50.5 & 15 & 0.57 & 70 & 405 & $(<2)$ & $(0.5)$ & $(0.07)$ & $(<1)$ \\
\hline 395 & 99.944 & 5.4 & 0.60 & 12.2 & 13 & 0.32 & 1.5 & 12.2 & $(<2)$ & $(0.4)$ & $(0.13)$ & $(<1)$ \\
\hline 396 & 99.955 & 4.2 & 0.50 & 3.30 & 9.5 & $<0.1$ & 0.8 & 5.0 & $(<2)$ & $(0.6)$ & $(<0.05)$ & $(<1)$ \\
\hline 398 & 99.98 & 7.0 & 14 & 20.1 & (11) & 11 & 4.8 & 24 & $(<2)$ & $(22)$ & $(0.1)$ & $(<1)$ \\
\hline 399 & 99.79 & 506 & $(-95)$ & 116.8 & $(10)$ & $(\sim 50)$ & $(-90)$ & 45 & $(<2)$ & $(<1)$ & (4) & $(<1)$ \\
\hline 400 & 99.70 & 603 & $(-250)$ & 181 & (9) & $(\sim 155)$ & $(\sim 200)$ & 114 & $(<2)$ & $(<1)$ & $(10)$ & $(<1)$ \\
\hline 454 & 99.84 & $(150)$ & 400 & 286 & & & 2.2 & 7 & & & 7.5 & \\
\hline
\end{tabular}

Values in parentheses are not certified, but are given for information only.

\begin{tabular}{|c|c|c|c|c|c|c|c|c|c|c|}
\hline SRM & 0 & Si & $\mathrm{Be}$ & B & $\mathrm{Ca}$ & $\mathrm{Li}$ & $\mathbf{P a}$ & $\mathbf{P}$ & $\mathrm{Ti}$ & $\mathrm{Zr}$ \\
\hline 393 & & $<0.5$ & $<0.01$ & $<0.01$ & $<0.05$ & $<0.01$ & $<0.05$ & $<0.05$ & $<0.5$ & $<0.5$ \\
\hline 394 & $(230)$ & $(<2)$ & & & & & & & & \\
\hline 395 & $(435)$ & $(<2)$ & & & & & & & & \\
\hline$\overline{396}$ & $(270)$ & $(<2)$ & & & & & & & & \\
\hline 398 & $(30)$ & $(<2)$ & & & & & & & & \\
\hline 399 & $(950)$ & $(<2)$ & & & & & & & & \\
\hline 400 & (1025) & $(<2)$ & & & & & & & & \\
\hline 454 & & & & & & & & & & \\
\hline
\end{tabular}

Values in parentheses are not certifed, but are given for information only. 


\section{Lead-Base Alloys}

\begin{tabular}{|c|c|c|c|c|c|c|c|c|c|c|}
\hline \multirow[b]{2}{*}{ SRM } & \multirow[b]{2}{*}{ Type } & \multirow[b]{2}{*}{$\begin{array}{l}\text { Wt/Unit } \\
\text { (grams) }\end{array}$} & \multirow[b]{2}{*}{$\begin{array}{l}\text { (Other } \\
\text { Forms) }\end{array}$} & \multicolumn{7}{|c|}{ Chemical Composition (Nominal Weight Percent) } \\
\hline & & & & $\mathrm{Cu}$ & $\mathrm{Ni}$ & As & Sn & Sb & $\mathrm{Bi}$ & $\mathrm{Fe}$ \\
\hline $53 \mathrm{e}$ & Bearing Metal (84Pb-10Sb-6Sn) & 150 & 1132 & 0.054 & 0.003 & 0.057 & 5.84 & 10.26 & 0.052 & $<0.001$ \\
\hline $127 \mathrm{~b}$ & Solder (40Sn-60Pb) & 150 & 1131 & 0.011 & 0.012 & 0.01 & 39.3 & 0.43 & 0.06 & $\overline{\mathrm{Ag} 0.01}$ \\
\hline
\end{tabular}

Magnesium-Base Alloys

\begin{tabular}{|cccccccccccc|}
\hline & & \multicolumn{8}{c|}{ Chemical Composition (Nominal Weight Percent) } \\
\cline { 4 - 12 } SRM & Type & $\begin{array}{c}\text { Wt/Unit } \\
\text { (grams) }\end{array}$ & Mn & Si & Cu & Ni & Al & Pb & Fe & Zn \\
\hline 171 & Alloy & 100 & 0.45 & 0.0118 & 0.0112 & 0.0009 & 2.98 & 0.0033 & 0.0018 & 1.05 \\
\hline
\end{tabular}

Nickel-Base Alloys

\begin{tabular}{|c|c|c|c|c|c|c|c|c|c|c|}
\hline \multirow[b]{2}{*}{ SRM } & \multirow[b]{2}{*}{1 Type } & \multirow[b]{2}{*}{$\begin{array}{l}\text { Wt/Unit } \\
\text { (grams) }\end{array}$} & \multicolumn{8}{|c|}{ Chemical Composition (Nominal Weight Percent) } \\
\hline & & & C & Mn & $\mathbf{P}$ & S & $\mathrm{Si}$ & $\mathrm{Cu}$ & $\mathbf{N i}$ & $\mathrm{Cr}$ \\
\hline 349 & $\mathrm{Ni57}-\mathrm{Cr} 20$ & 150 & 0.08 & 0.43 & 0.002 & $<0.01$ & 0.29 & 0.006 & 57.15 & 19.50 \\
\hline 882 & Ni66-Cu31-A13 & 150 & 0.006 & 0.0007 & & 0.0014 & 0.006 & 31.02 & 65.25 & \\
\hline 864 & Inconel, 600 & & 0.06 & 0.3 & $\langle 0.01$ & $<0.01$ & 0.1 & 0.3 & 72.6 & 16 \\
\hline$\overline{865}$ & Inconel, 625 & & 0.04 & 0.2 & 0.01 & $\langle 0.01$ & 0.4 & 0.4 & 60 & 22 \\
\hline 866 & Incoloy, 800 & & 0.08 & 0.9 & 0.02 & $\langle 0.01$ & 0.1 & 0.5 & 30 & 20 \\
\hline$\overline{867}$ & Incoloy, 825 & & 0.02 & 0.4 & 0.02 & $\langle 0.01$ & 0.3 & 1.6 & 43 & 23 \\
\hline
\end{tabular}

\begin{tabular}{|c|c|c|c|c|c|c|c|c|c|c|c|}
\hline SRM & $\mathbf{V}$ & Mo & $\mathbf{W}$ & Co & Ti & $\mathrm{Al}$ & B & $\mathrm{Fe}$ & $\mathrm{Nb}$ & $\mathrm{Ta}$ & $\mathrm{Zr}$ \\
\hline 349 & 0.081 & 4.04 & $<0.01$ & 13.95 & 3.05 & 1.23 & 0.0046 & 0.13 & $<0.01$ & $<0.01$ & 0.081 \\
\hline$\overline{882}$ & & & & & 0.57 & 2.85 & & 0.009 & & & \\
\hline 864 & & 0.2 & & 0.06 & 0.3 & 0.3 & $\langle 0.01$ & 9 & 0.1 & & \\
\hline$\overline{865}$ & & 8.7 & & 0.1 & 0.3 & 0.3 & $<0.01$ & 4 & 3.5 & & \\
\hline$\overline{866}$ & & 0.3 & & 0.08 & 0.3 & 0.3 & $\langle 0.01$ & 47 & 0.1 & & \\
\hline 867 & & 2.7 & & 0.08 & 0.7 & 0.06 & $\langle 0.01$ & 27 & 0.4 & & \\
\hline
\end{tabular}




\section{Trace Elements in Nickel-Base Superalloy}

The Gas Turbine Panel of the ASTM-ASME-MPG Joint Committee, through recommendation of its Task Force on Trace Elements in Superalloys, prepared a set of three "Tracealloy" materials, which have the same common matrix. These were given to NBS for "definitive analysis" of: $\mathrm{Pb}, \mathrm{Bi}, \mathrm{Se}, \mathrm{Te}$, and Tl; trace elements that vary over the concentration range of interest. These SRM's 897,898 , and 899 are in the form of fine particles.

\begin{tabular}{lllllll}
\hline & & \multicolumn{4}{c}{ Nominal Trace Composition (Parts Per Million by Weight) } \\
\cline { 3 - 7 } SRM & Type & Pb & Bi & Se & Te & Tl \\
\hline 897 & "Tracealloy" A & 11.7 & $(0.5)$ & 9.1 & 1.05 & 0.51 \\
\hline 898 & "Tracealloy" B & 2.5 & $(1.0)$ & 2.00 & 0.54 & 2.75 \\
\hline 899 & "Tracealloy" C & 3.9 & $(0.3)$ & 9.5 & 5.9 & 0.252 \\
\hline
\end{tabular}

\begin{tabular}{cccccccccccccc|}
\hline & \multicolumn{110}{c|}{ Approximate Base Composition (Weight Percent) } \\
\cline { 2 - 14 } & $\mathbf{C}$ & $\mathbf{C r}$ & $\mathbf{C o}$ & $\mathbf{N i}$ & $\mathbf{W}$ & $\mathbf{N b}$ & $\mathbf{A l}$ & $\mathbf{T i}$ & $\mathbf{B}$ & $\mathbf{Z r}$ & Ta & Hf \\
\hline 897 & $(0.12)$ & $(12.0)$ & $(8.5)$ & $(\mathrm{Bal})$ & $(1.75)$ & $(0.9)$ & $(2.0)$ & $(2.0)$ & $(0.010)$ & $(0.10)$ & $(1.75)$ & $(1.2)$ \\
\hline 898 & $(0.12)$ & $(12.0)$ & $(8.5)$ & $(\mathrm{Bal})$ & $(1.75)$ & $(0.9)$ & $(2.0)$ & $(2.0)$ & $(0.010)$ & $(0.10)$ & $(1.75)$ & $(1.2)$ \\
\hline 899 & $(0.12)$ & $(12.0)$ & $(8.5)$ & $(\mathrm{Bal})$ & $(1.75)$ & $(0.9)$ & $(2.0)$ & $(2.0)$ & $(0.010)$ & $(0.10)$ & $(1.75)$ & $(1.2)$ \\
\hline
\end{tabular}

Values in parentheses are not certified, but are given for information only.

\section{Nickel Oxide}

The nickel oxide SRM's are available primarily for application in the electronics industry to the analysis of cathode grade nickel. The "Standard Method for Spectrochemical Analysis of Thermionic Nickel Alloys by the Powder-DC Arc Technique." ASTM Designation E129, is based on calibration with these standards. The values given are for the percentage of the element in nickel oxide.

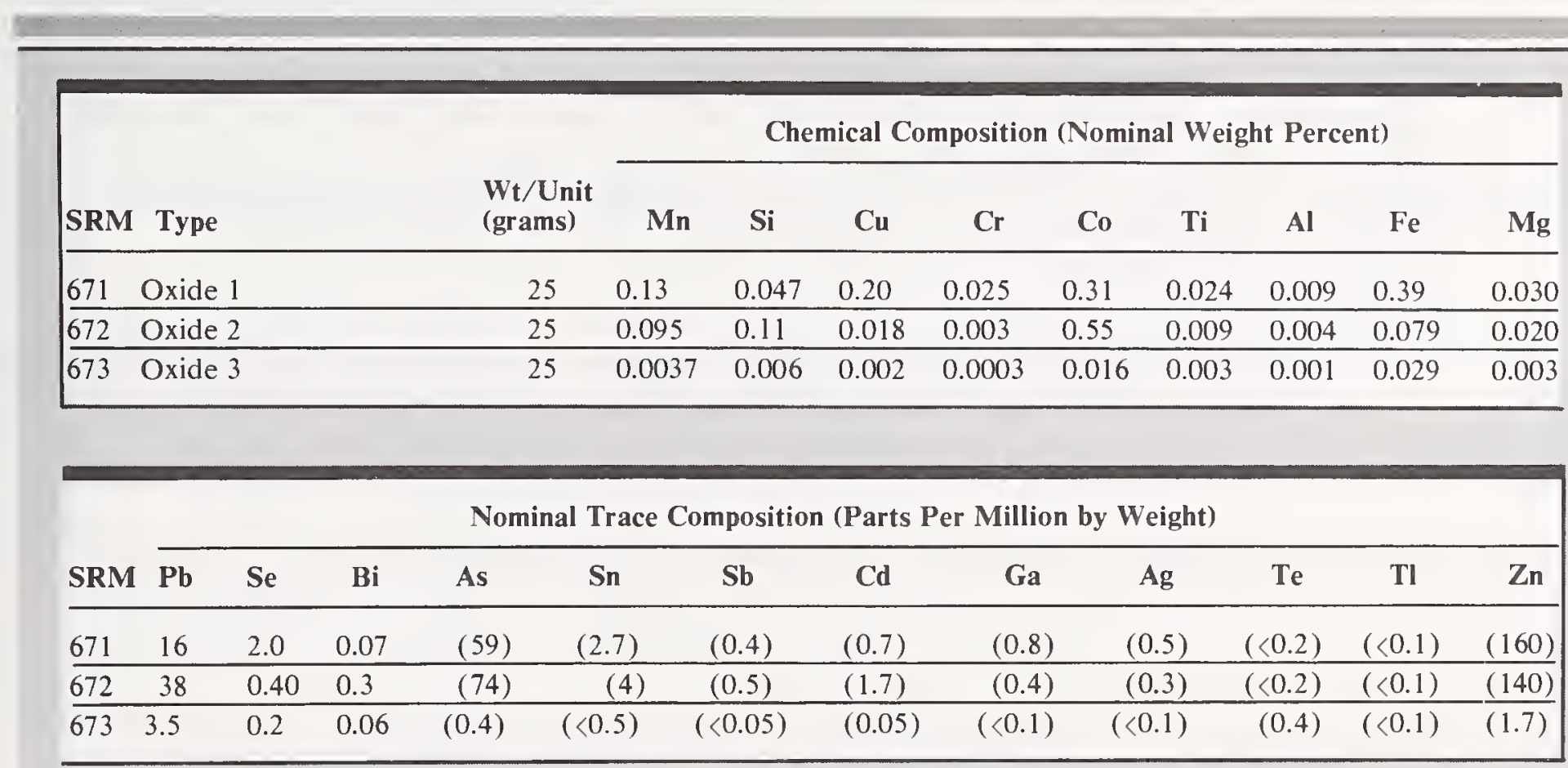

Values in parentheses are not certified, but are given for information only. 


\section{Selenium}

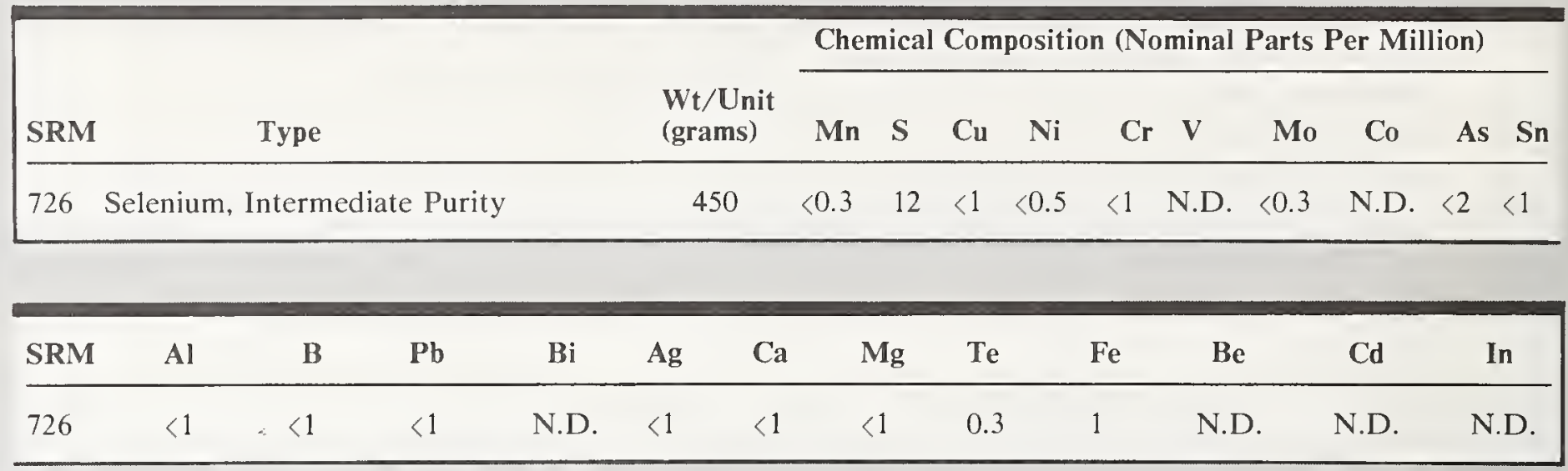

N.D. $=$ Not detected at limits of detection of $<0.3 \mathrm{ppm}$.

\section{Tin-Base Alloys}

\begin{tabular}{|c|c|c|c|c|c|c|c|c|c|c|c|}
\hline \multirow[b]{2}{*}{ SRM } & \multirow[b]{2}{*}{ Type } & \multirow[b]{2}{*}{$\begin{array}{l}\text { Wt/Unit } \\
\text { (grams) }\end{array}$} & \multicolumn{9}{|c|}{ Chemical Composition (Nominal Weight Percent) } \\
\hline & & & $\mathrm{Pb}$ & $\mathrm{Sn}$ & $\mathrm{Sb}$ & $\mathrm{Bi}$ & $\mathrm{Cu}$ & $\mathrm{Fe}$ & As & $\mathrm{Ag}$ & $\mathrm{Ni}$ \\
\hline $54 d$ & Bearing Metal & 170 & 0.62 & 88.57 & 7.04 & 0.044 & 3.62 & 0.027 & 0.088 & 0.0032 & 0.0027 \\
\hline
\end{tabular}

\begin{tabular}{|c|c|c|c|c|c|c|c|c|c|c|}
\hline \multirow[b]{2}{*}{ SRM } & \multirow[b]{2}{*}{ Type } & \multirow[b]{2}{*}{$\begin{array}{l}\text { Wt/Unit } \\
\text { (grams) }\end{array}$} & \multicolumn{8}{|c|}{ Chemical Composition (Nominal Weight Percent) } \\
\hline & & & $\mathrm{C}$ & $\mathrm{Mn}$ & $\mathrm{Cu}$ & Mo & Sn & $\mathrm{Al}$ & $\mathrm{Fe}$ & $\mathbf{N}$ \\
\hline $173 b$ & 6A1-4V (IN PREP) & & & & & & & & & \\
\hline 176 & $5 \mathrm{Al}-2.5 \mathrm{Sn}$ & 100 & 0.015 & 0.0008 & 0.003 & 0.0003 & 2.47 & 5.16 & 0.070 & 0.010 \\
\hline 650 & Unalloyed (IN PREP) & & & & & & & & & \\
\hline 651 & Unalloyed (IN PREP) & & & & & & & & & \\
\hline 652 & Unalloyed (IN PREP) & & & & & & & & & \\
\hline
\end{tabular}




\section{Zinc-Base Alloys}

\begin{tabular}{|c|c|c|c|c|c|c|c|c|c|c|c|}
\hline \multirow[b]{2}{*}{ SRM } & \multirow[b]{2}{*}{ Type } & \multirow[b]{2}{*}{$\begin{array}{l}\text { Wt/Unit } \\
\text { (grams) }\end{array}$} & \multicolumn{9}{|c|}{ Chemical Composition (Nominal Weight Percent) } \\
\hline & & & Mn & $\mathrm{Cu}$ & $\mathrm{Ni}$ & Sn & Al & $\mathrm{Cd}$ & $\mathrm{Fe}$ & $\mathbf{P b}$ & $\mathrm{Ag}$ \\
\hline $94 c$ & $\begin{array}{c}\text { Die Casting } \\
\text { Alloy }\end{array}$ & 150 & 0.014 & 1.01 & 0.006 & 0.006 & 4.13 & 0.002 & 0.018 & 0.006 & $\mathrm{Mg} 0.042$ \\
\hline 728 & Zinc & 450 & & 0.00057 & & $(0.000002)$ & & 0.000115 & 0.00027 & 0.00111 & 0.00011 \\
\hline
\end{tabular}

\section{Zirconium-Base Alloys}

\begin{tabular}{|c|c|c|c|c|c|c|c|c|c|c|c|c|c|}
\hline \multirow[b]{2}{*}{ SRM } & \multirow[b]{2}{*}{ Type } & \multirow{2}{*}{$\begin{array}{l}\text { Wt/Unit } \\
\text { (grams) }\end{array}$} & \multicolumn{11}{|c|}{ Chemical Composition (Nominal Parts Per Million) } \\
\hline & & & C & Mn & $\mathrm{Si}$ & $\mathrm{Cu}$ & $\mathbf{N i}$ & $\mathrm{Cr}$ & $\mathrm{Ti}$ & $\operatorname{Sn}(W t \%)$ & $\mathrm{Fe}$ & $\mathbf{N}$ & U \\
\hline $360 a$ & Zircaloy-2 & 100 & 136 & 3 & 51 & 140 & 554 & 1060 & 27 & 1.42 & 1441 & 43 & 0.15 \\
\hline
\end{tabular}

\section{Nonferrous Alloys [Solid Form]}

These SRM's are designed to fill the basic needs of the nonferrous primary and secondary metals industries for analytical control, primarily with optical emission and $\mathrm{x}$-ray spectroscopic methods. Both nominal chemical composition and analytical range SRM's have been prepared for many of the commercially important nonferrous alloy systems.

\section{Aluminum "Benchmark"}

Aluminum "benchmark" standards were prepared in a cooperative Industry-ASTM-NBS program. These SRM's are intended for use primarily in optical emission and x-ray spectrometric methods of analysis. These materials are also available in the form of chips, for checking chemical methods of analysis (see page 39).

\begin{tabular}{|c|c|c|c|c|c|c|c|c|c|c|c|}
\hline \multirow[b]{2}{*}{ SRM } & \multirow[b]{2}{*}{ Type } & \multirow[b]{2}{*}{ Size } & \multicolumn{9}{|c|}{ Chemical Composition (Nominal Weight Percent) } \\
\hline & & & $\mathbf{S i}$ & $\mathrm{Fe}$ & $\mathrm{Cu}$ & Mn & $\mathrm{Cr}$ & $\mathbf{N i}$ & $\mathrm{Zn}$ & $\mathbf{M g}$ & $\mathrm{Be}$ \\
\hline 1258 & Alloy 6011 & $\begin{array}{c}35 \mathrm{~mm} \mathrm{D} \mathrm{X} \\
19 \mathrm{~mm} \text { thick }\end{array}$ & 0.78 & 0.079 & 0.84 & 0.48 & 0.0011 & 0.0006 & 1.03 & 0.98 & $<0.0001$ \\
\hline 1259 & Alloy 7075 & $\begin{array}{l}35 \mathrm{~mm} \mathrm{D} \mathrm{X} \\
19 \mathrm{~mm} \text { thick }\end{array}$ & 0.18 & 0.205 & 1.60 & 0.079 & 0.173 & 0.063 & 5.44 & 2.48 & 0.0025 \\
\hline
\end{tabular}




\section{Copper-Base Alloys}

A number of copper-base alloy SRM's were prepared to provide for analytical control by rapid instrumental methods in the copper industry. These SRM's are for calibration of optical emission and x-ray spectroscopic equipment. Eight groups were prepared in two forms: chill-cast (with "C" prefix) for the producer (blocks, $31 \mathrm{~mm}$ square, $19 \mathrm{~mm}$ thick), and wrought for the consumer (disks, $31 \mathrm{~mm}$ in diameter and $19 \mathrm{~mm}$ thick). Both forms have nearly identical chemical compositions. Consequently, when the supply of one form is exhausted, the other is the recommended replacement. For each of the eight principal copper-base alloys, three SRM's were prepared to comprise a "nominal-composition," and both a low- and high-composition standard. To make the cartridge-brass SRM's more widely applicable, a number of trace elements were purposely added and certified. The beryllium copper SRM's are representative of the nominal chemical composition of three Copper and Brass Research Association (CABRA) alloy designations.

\begin{tabular}{|c|c|c|c|c|c|c|c|c|c|c|}
\hline \multirow{2}{*}{\multicolumn{2}{|c|}{ SRM }} & \multirow[b]{2}{*}{ Туре } & \multicolumn{8}{|c|}{ Chemical Composition (Nominal Weight Percent) } \\
\hline & & & $\mathrm{Cu}$ & $\mathrm{Zn}$ & $\mathrm{Pb}$ & $\mathrm{Fe}$ & Sn & $\mathrm{Ni}$ & Al & $\mathbf{S b}$ \\
\hline 1102 & & Cartridge Brass $\mathrm{C}$ & 72.85 & 27.10 & 0.020 & 0.011 & 0.006 & 0.005 & 0.007 & 0.005 \\
\hline \multirow[t]{2}{*}{1103} & & Free-Cutting Brass A & 59.27 & 35.72 & 3.73 & 0.26 & 0.88 & 0.15 & & \\
\hline & C1104 & Free-Cutting Brass B & 61.33 & 35.31 & 2.77 & 0.088 & 0.43 & 0.070 & & \\
\hline 1106 & C1106 & Naval Brass A & 59.08 & 40.08 & 0.032 & 0.004 & 0.74 & 0.025 & & \\
\hline 1107 & $\mathrm{C} 1107$ & Naval Brass B & 61.21 & 37.34 & 0.18 & 0.037 & 1.04 & 0.098 & & \\
\hline 1108 & C1108 & Naval Brass $\mathrm{C}$ & 64.95 & 34.42 & 0.063 & 0.050 & 0.39 & 0.033 & & \\
\hline 1109 & C1109 & Red Brass A & 82.2 & 17.43 & 0.075 & 0.053 & 0.10 & & & \\
\hline 1110 & $\mathrm{C} 1110$ & Red Brass B & 84.59 & 15.20 & 0.033 & 0.033 & 0.051 & 0.053 & & \\
\hline 1111 & C1111 & Red Brass C & 87.14 & 12.81 & 0.013 & 0.010 & 0.019 & 0.022 & & \\
\hline 1112 & $\mathrm{C} 1112$ & Gilding Metal A & 93.38 & 6.30 & 0.057 & 0.070 & 0.12 & 0.100 & & \\
\hline 1113 & $\mathrm{C} 1113$ & Gilding Metal B & 95.03 & 4.80 & 0.026 & 0.043 & 0.064 & 0.057 & & \\
\hline 1114 & C1114 & Gilding Metal C & 96.45 & 3.47 & 0.012 & 0.017 & 0.027 & 0.021 & & \\
\hline 1115 & C1115 & Commercial Bronze A & 87.96 & 11.73 & 0.013 & 0.13 & 0.10 & 0.074 & & \\
\hline 1116 & C1116 & Commercial Bronze B & 90.37 & 9.44 & 0.042 & 0.046 & 0.044 & 0.048 & & \\
\hline 1117 & $\mathrm{C} 1117$ & Commercial Bronze $\mathrm{C}$ & 93.01 & 6.87 & 0.069 & 0.014 & 0.021 & 0.020 & & \\
\hline 1118 & C1118 & Aluminum Brass $\mathrm{A}$ & 75.1 & 21.9 & 0.025 & 0.065 & & & 2.80 & 0.010 \\
\hline 1119 & C1119 & Aluminum Brass B & 77.1 & 20.4 & 0.050 & 0.030 & & & 2.14 & 0.050 \\
\hline \multirow[t]{2}{*}{1122} & $\mathrm{C} 1122$ & Beryllium Copper CA-170 & 97.45 & $(0.01)$ & $(0.003)$ & 0.16 & $(0.01)$ & $(0.01)$ & 0.17 & \\
\hline & $\mathrm{C} 1123$ & Beryllium Copper CA-175 & 97.10 & 0.01 & $(0.001)$ & 0.04 & $(0.01)$ & $(0.01)$ & 0.02 & \\
\hline 1275 & & Cupro-Nickel (CDA 706) & 88.2 & 0.085 & 0.006 & 1.46 & 0.008 & 9.76 & & 0.0005 \\
\hline 1276 & & Cupro-Nickel (CDA 715) & 67.8 & 0.038 & 0.004 & 0.56 & 0.023 & 30.5 & & 0.0004 \\
\hline
\end{tabular}


Copper Base Alloys (Continued)

\begin{tabular}{|c|c|c|c|c|c|c|c|c|c|}
\hline \multicolumn{2}{|c|}{ SRM } & As & $\mathrm{Be}$ & $\mathbf{B i}$ & $\mathrm{Cd}$ & $\mathrm{Mn}$ & $\mathbf{P}$ & $\mathrm{Si}$ & $\mathbf{A g}$ \\
\hline \multicolumn{2}{|l|}{1102} & 0.004 & 0.00003 & 0.0005 & 0.0045 & 0.0045 & 0.0048 & $(0.002)$ & 0.0010 \\
\hline \multicolumn{2}{|l|}{1103} & & & & & & 0.003 & & \\
\hline & $\mathrm{C} 1104$ & & & & & & 0.005 & & \\
\hline 1106 & C1106 & & & & & 0.005 & & & \\
\hline 1107 & C1107 & & & & & & & & \\
\hline$\overline{1108}$ & C1108 & & & & & 0.025 & & & \\
\hline 1109 & C1109 & & & & & & 0.006 & & \\
\hline$\overline{1110}$ & C1110 & & & & & & & & \\
\hline 1111 & $\mathrm{C} 1111$ & & & & & & & & \\
\hline 1112 & $\mathrm{C} 1112$ & & & & & & 0.009 & & \\
\hline 1113 & $\mathrm{C} 1113$ & & & & & & 0.008 & & \\
\hline$\overline{1114}$ & C1114 & & & & & & 0.009 & & \\
\hline 1115 & C1115 & & & & & & 0.005 & & \\
\hline$\overline{1116}$ & C1116 & & & & & & 0.008 & & \\
\hline 1117 & C1117 & & & & & & 0.002 & & \\
\hline$\overline{1118}$ & C1118 & 0.007 & & & & & 0.13 & 0.0021 & \\
\hline 1119 & C1119 & 0.040 & & & & & 0.070 & 0.0015 & \\
\hline \multirow[t]{2}{*}{1122} & $\mathrm{C} 1122$ & & 1.75 & & & $(0.004)$ & $(0.004)$ & 0.17 & $(0.005)$ \\
\hline & C1123 & & 0.46 & & & $(0.002)$ & $(0.002)$ & 0.03 & $(0.009)$ \\
\hline \multirow{2}{*}{\multicolumn{2}{|c|}{1275}} & $(0.001)$ & & $(<0.001)$ & 0.0003 & 0.42 & 0.005 & $(0.001)$ & $(0.004)$ \\
\hline & & $(<0.001)$ & & $(<0.0001)$ & 0.0002 & 1.01 & 0.006 & $(0.001)$ & $(0.004)$ \\
\hline
\end{tabular}

Values in parentheses are not certified, but are given for information only.

\begin{tabular}{|c|c|c|c|c|c|c|c|c|c|}
\hline \multicolumn{2}{|c|}{ SRM } & $\mathrm{Te}$ & Co & $\mathrm{Cr}$ & Se & $\mathbf{M g}$ & B & $S$ & $\mathrm{Ti}$ \\
\hline \multirow[t]{3}{*}{1102} & & 0.0003 & & & & & & & \\
\hline & $\mathrm{C} 1103$ & & & & & & & & \\
\hline & $\mathrm{C} 1104$ & & & & & & & & \\
\hline 1106 & C1106 & & & & & & & & \\
\hline 1107 & C1107 & & & & & & & & \\
\hline 1108 & C1108 & & & & & & & & \\
\hline 1109 & C1109 & & & & & & & & \\
\hline 1110 & C1110 & & & & & & & & \\
\hline 1111 & $\mathrm{C} 1111$ & & & & & & & & \\
\hline 1112 & $\mathrm{C} 1112$ & & & & & & & & \\
\hline 1113 & $\mathrm{C} 1113$ & & & & & & & & \\
\hline 1114 & $\mathrm{C} 1114$ & & & & & & & & \\
\hline 1115 & $\mathrm{C} 1115$ & & & & & & & & \\
\hline 1116 & C1116 & & & & & & & & \\
\hline 1117 & $\mathrm{C} 1117$ & & & & & & & & \\
\hline \multicolumn{10}{|c|}{$1118 \quad \mathrm{C} 1118$} \\
\hline \multicolumn{10}{|c|}{$1119 \quad \mathrm{C} 1119$} \\
\hline \multirow[t]{2}{*}{1122} & $\mathrm{C} 1122$ & & 0.220 & $(0.002)$ & & & & & \\
\hline & C1123 & & 2.35 & $(0.001)$ & & & & & \\
\hline 1275 & & $(0.0002)$ & 0.024 & $(0.0002)$ & 0.0004 & 0.003 & $(0.0009)$ & $(0.008)$ & $(0.0002)$ \\
\hline 1276 & & $(0.0002)$ & 0.045 & $(0.0002)$ & 0.0005 & 0.12 & $(0.0001)$ & $(0.008)$ & $(0.0002)$ \\
\hline
\end{tabular}




\section{Copper" Benchmark"}

The copper "benchmark" standards were prepared in a cooperative Industry-ASTM-NBS program and were designed primarily for use in calibration with optical emission methods of analysis. They should also serve in the development of other new or improved trace methods of analysis. Twelve different compositions are issued as 25 SRM's Cu "O" and $\mathrm{Cu} \mathrm{XI}$ is issued in chip form only. Cu IV is available only in rod form 6.60 $\mathrm{mm}$ in diameter and $103 \mathrm{~mm}$ long. Cu I, II, III, V, VI, and VII are available both as chips and as rods 6.35 $\mathrm{mm}$ in diameter and $103 \mathrm{~mm}$ long. Cu VIII, IX, and X are issued as chill-cast and unidirectionally solidified blocks $32 \mathrm{~mm}$ square and $19 \mathrm{~mm}$ thick, $\mathrm{Cu}$ VIII. $\mathrm{Cu} \mathrm{X}$ are phosphorized copper containing a nominal concentration range from about 10 to $500 \mathrm{ppm}$ for the same 20 trace elements contained in the other copper "benchmark" SRM's, plus five to eight additional elements. These SRM's are also applicable for x-ray fluorescence methods of analysis and, because of deliberate additions of gold and silver (in ratios of 1 to 4), for calibration of fire assay equipment.

\begin{tabular}{|c|c|c|c|c|c|c|c|}
\hline \multirow[b]{2}{*}{ SRM } & \multirow[b]{2}{*}{ Type } & \multirow[b]{2}{*}{ Form } & \multicolumn{5}{|c|}{ Chemical Composition (Nominal Parts Per Million) } \\
\hline & & & $\mathrm{Cu}(\mathrm{Wt} \%)$ & $\mathbf{S b}$ & As & $\mathbf{B i}$ & $\mathbf{F e}$ \\
\hline 494 & Unalloyed-Cu I & rod & 99.91 & 4.5 & 2.6 & 0.35 & $(-155)$ \\
\hline 495 & Unalloyed-Cu II & rod & 99.94 & 8.0 & 1.6 & 0.50 & $(\sim 100)$ \\
\hline 496 & Unalloyed-Cu III & rod & 99.95 & $<1$ & $<0.2$ & 0.07 & $(-150)$ \\
\hline 457 & Unalloyed-Cu IV & rod & 99.96 & 0.2 & 0.2 & 0.2 & 2.0 \\
\hline 498 & Unalloyed-Cu V & rod & 99.98 & 7.4 & 25 & 2.0 & 11 \\
\hline 499 & Unalloyed-Cu VI & rod & 99.79 & 30 & 47 & 10.5 & 21 \\
\hline 500 & Unalloyed-Cu VII & rod & 99.70 & 100 & 140 & 25 & 42 \\
\hline $\mathrm{C} 1251$ & Unalloyed-Cu VIII & disk & 99.96 & 12.6 & $(8)$ & (3) & $(10)$ \\
\hline C1252 & Unalloyed-Cu IX & disk & 99.89 & 42 & 124 & 20 & $(40)$ \\
\hline C1253 & Unalloyed-Cu X & disk & 99.42 & $(132)$ & 244 & 70 & $(300)$ \\
\hline SRM & $\mathbf{M n}$ & $\mathrm{Ni}$ & Se & Ag & Te & Sn & $\mathrm{Zn}$ \\
\hline 494 & 26.5 & 11.7 & 2.1 & 50 & 0.6 & 70 & 400 \\
\hline 495 & 5.3 & 5.4 & 0.6 & 12.2 & 0.3 & 1.5 & 12 \\
\hline 496 & 0.4 & 4.2 & 0.5 & 3.3 & $<0.1$ & 0.8 & 5.0 \\
\hline 457 & $\therefore \quad<0.1$ & 0.6 & 3.3 & 8.1 & 0.5 & $<0.2$ & $<11$ \\
\hline 498 & $(0.3)$ & 7.0 & 14 & 20.1 & 11 & 5 & 25 \\
\hline 499 & $(0.3)$ & 504 & $(-90)$ & 114 & $(-50)$ & $(-90)$ & 41 \\
\hline 500 & $(0.2)$ & 603 & $(-250)$ & 176 & $(-155)$ & $(-200)$ & 111 \\
\hline C1251 & (7) & 22 & 8.6 & 81.4 & $(12)$ & $(15)$ & 8.3 \\
\hline C1252 & $(28)$ & 128 & 46 & 166.6 & $(44)$ & $(124)$ & 60 \\
\hline C1253 & {$[\sim 300]$} & $(500)$ & 140 & 503 & (193) & $(489)$ & 368 \\
\hline
\end{tabular}




\section{Copper "Benchmark" (Continued)}

\begin{tabular}{lcccccccr|}
\hline SRM & Al & Cd & Cr & Co & Au & Mg & O & S \\
\hline 494 & $(<2)$ & $(0.5)$ & 2.0 & 0.5 & $(0.07)$ & $(<1)$ & $(230)$ & 15 \\
\hline 495 & $(<2)$ & $(0.4)$ & 6.0 & 0.3 & $(0.13)$ & $(<1)$ & $(435)$ & 13 \\
\hline 496 & $(<2)$ & $(0.6)$ & 4.3 & 0.4 & $(<0.05)$ & $(<1)$ & $(270)$ & 9 \\
\hline 457 & $(<2)$ & $(<1)$ & $(0.3)$ & $(0.2)$ & $(<0.05)$ & $(<1)$ & $(360)$ & $(4)$ \\
\hline 498 & $(<2)$ & $(22)$ & $(0.3)$ & 2.7 & $(0.1)$ & $(<1)$ & $(30)$ & $(11)$ \\
\hline & & & & & & & & \\
\hline 499 & $(<2)$ & $(<1)$ & $(0.5)$ & 0.5 & $(4)$ & $(<1)$ & $(950)$ & $(10)$ \\
\hline 500 & $(<2)$ & $(<1)$ & $(0.5)$ & 0.5 & $(10)$ & $(<1)$ & $(1025)$ & $(9)$ \\
\hline $\mathrm{C} 1251$ & {$[\sim 5]$} & {$[\sim 3]$} & 2.8 & 8.8 & 15.0 & $(10)$ & {$[\sim 120]$} & $(22)$ \\
\hline $\mathrm{C} 1252$ & {$[\sim 40]$} & {$[\sim 15]$} & 7.4 & 90 & 34.9 & $(20)$ & {$[\sim 150]$} & $(29)$ \\
\hline $\mathrm{C} 1253$ & {$[\sim 160]$} & {$[\sim 60]$} & $(187)$ & $(510)$ & 74.4 & $(80)$ & {$[\sim 85]$} & $(50)$ \\
\hline
\end{tabular}

Values in parentheses are not certified, but are given for information only.

Figures in brackets are qualitative estimates only.

\section{Lead-Base Alloys}

SRM 31.4

$\mathrm{mm} \mathrm{D} \times 19$

mm thick
Chemical Composition (Nominal Weight Percent)

\begin{tabular}{|lcccccccccc} 
mm thick & \multicolumn{1}{c}{ Type } & Other Forms & $\mathrm{Cu}$ & $\mathrm{Ni}$ & $\mathrm{As}$ & $\mathrm{Sn}$ & $\mathrm{Sb}$ & $\mathrm{Bi}$ & $\mathrm{Ag}$ & $\mathrm{Fe}$ \\
\hline 1131 & Solder Pb60-Sn40 & $127 \mathrm{~b}$ & 0.011 & 0.012 & 0.01 & 39.3 & 0.43 & 0.06 & 0.01 & \\
\hline 1132 & Bearing Metal & $53 \mathrm{e}$ & 0.054 & 0.003 & 0.057 & 5.84 & 10.2 & 0.052 & & $<0.001$ \\
\hline
\end{tabular}

\section{Nickel-Base Alloys}

\section{SRM $31 \mathrm{~mm}$}

$\mathrm{D} \times 19 \mathrm{~mm}$

thick

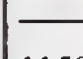

1159

1160
Ni48, balance Fe

Ni80, Mo4, balance Fe
Chemical Composition (Nominal Weight Percent)

\begin{tabular}{lllllllllll}
\hline $\mathrm{C}$ & $\mathrm{Mn}$ & $\mathrm{P}$ & $\mathrm{S}$ & $\mathrm{Si}$ & $\mathrm{Cu}$ & $\mathrm{Ni}$ & $\mathrm{Cr}$ & $\mathrm{Mo}$ & $\mathrm{Co}$ & $\mathrm{Fe}$
\end{tabular}

$\begin{array}{lllllllllll}0.007 & 0.305 & 0.003 & 0.003 & 0.32 & 0.038 & 48.2 & 0.06 & 0.010 & 0.022 & 51.0\end{array}$

$\begin{array}{llllllllllll}0.019 & 0.550 & 0.003 & 0.001 & 0.37 & 0.021 & 80.3 & 0.05 & 4.35 & 0.054 & 14.3\end{array}$




\section{Titanium-Base Alloys}

\begin{tabular}{|c|c|c|c|c|c|c|c|}
\hline \multirow{2}{*}{$\begin{array}{l}\text { SRM } 31 \mathrm{~mm} \\
\mathrm{D} \times 19 \mathrm{~mm} \\
\text { thick }\end{array}$} & \multirow[b]{2}{*}{ Type } & \multicolumn{6}{|c|}{ Chemical Composition (Nominal Weight Percent) } \\
\hline & & Mn & $\mathrm{Cr}$ & $\mathrm{Fe}$ & Mo & $\mathrm{Al}$ & v \\
\hline 641 & $8 \mathrm{Mn}(\mathrm{A})$ & 6.68 & & & & & \\
\hline$\overline{642}$ & $8 \mathrm{Mn}(\mathrm{B})$ & 9.08 & & & & & \\
\hline 643 & $8 \mathrm{Mn}(\mathrm{C})$ & 11.68 & & & & & \\
\hline 644 & $2 \mathrm{Cr}-2 \mathrm{Fe} \cdot 2 \mathrm{Mo}(\mathrm{A})$ & & 1.03 & 1.36 & 3.61 & & \\
\hline 645 & $2 \mathrm{Cr}-2 \mathrm{Fe}-2 \mathrm{Mo}(\mathrm{B})$ & & 1.96 & 2.07 & 2.38 & & \\
\hline 646 & $2 \mathrm{Cr}-2 \mathrm{Fe}-2 \mathrm{Mo}(\mathrm{C})$ & & 3.43 & 2.14 & 1.11 & & \\
\hline $654 a^{*}$ & $6 \mathrm{Al}-4 \mathrm{~V}(\mathrm{~B})$ & $(<0.1)$ & $(0.20)$ & $(0.20)$ & $(<0.05)$ & $6.3_{4}$ & $3.9_{5}$ \\
\hline
\end{tabular}

*31 mm D $\times 6.4 \mathrm{~mm}$ thick.

Values in parentheses are not certified, but are given for information only.

\section{Zinc-Base Alloys}

Zinc-base alloy SRM's are available ranging from very high-purity zinc to commercial materials such as spelter and die-casting alloy compositions. They are supplied as bar segments (disks) intended for calibrating and checking optical emission and x-ray spectroscopic techniques. The certificate of analysis supplied with each gives the chemical composition determined at NBS and values determined by other laboratories that have cooperated in the certification of the SRM's. For high-purity zinc, see High-Purity Metals, page 52.

\begin{tabular}{|c|c|c|c|c|c|c|c|c|c|}
\hline \multirow[b]{2}{*}{ SRM } & \multirow[b]{2}{*}{ Type } & \multicolumn{8}{|c|}{ Chemical Composition (Nominal Weight Percent) } \\
\hline & & $\mathrm{Cu}$ & Al & Mg & $\mathrm{Fe}$ & $\mathbf{P b}$ & Cd & Sn & $\mathrm{Cr}$ \\
\hline 625 & Zinc-base A-ASTM AG 40A & 0.034 & 3.06 & 0.070 & 0.036 & 0.0014 & 0.0007 & 0.0006 & 0.0128 \\
\hline 626 & Zinc-base B-ASTM AG 40A & 0.056 & 3.56 & 0.020 & 0.103 & 0.0022 & 0.0016 & 0.0012 & 0.0395 \\
\hline 627 & Zinc-base C-ASTM AG 40A & 0.132 & 3.88 & 0.030 & 0.023 & 0.0082 & 0.0051 & 0.0042 & 0.0038 \\
\hline 628 & Zinc-base D-ASTM AC 41A & 0.611 & 4.59 & 0.0094 & 0.066 & 0.0045 & 0.0040 & 0.0017 & 0.0087 \\
\hline 629 & Zinc-base E-ASTM AC 41A & 1.50 & 5.15 & 0.094 & 0.017 & 0.0135 & 0.0155 & 0.012 & 0.008 \\
\hline 630 & Zinc-base F-ASTM AC 41A & 0.976 & 4.30 & 0.030 & 0.023 & 0.0083 & 0.0048 & 0.0040 & 0.0031 \\
\hline$\overline{631}$ & Zinc spelter (modified) & 0.0013 & 0.50 & $(<0.001)$ & 0.005 & $(0.001)$ & 0.0002 & 0.0001 & 0.0001 \\
\hline
\end{tabular}

\begin{tabular}{|c|c|c|c|c|c|c|c|c|}
\hline SRM & $\mathbf{M n}$ & $\mathrm{Ni}$ & $\mathrm{Si}$ & In & Ga & $\mathrm{Ca}$ & Ag & $\mathrm{Ge}$ \\
\hline 625 & 0.031 & 0.0184 & 0.017 & & & & & \\
\hline 626 & 0.048 & 0.047 & 0.042 & & & & & \\
\hline 627 & 0.014 & 0.0029 & 0.021 & & & & & \\
\hline 628 & 0.0091 & 0.030 & 0.008 & & & & & \\
\hline 629 & 0.0017 & 0.0075 & 0.078 & & & & & \\
\hline 630 & 0.0106 & 0.0027 & 0.022 & & & & & \\
\hline$\overline{631}$ & 0.00015 & $(<0.0005)$ & $(0.002)$ & 0.0023 & $(0.002)$ & $<0.001$ & $(<0.0005)$ & $(0.0002)$ \\
\hline
\end{tabular}

Values in parentheses are not certified, but are given for information only. 


\section{Zirconium-Base Alloys}

\begin{tabular}{|c|c|c|c|c|c|c|c|c|c|c|c|c|c|}
\hline \multirow{2}{*}{$\begin{array}{l}\text { SRM } 31 \mathrm{~mm} \\
\text { D } \times 9.5 \mathrm{~mm} \\
\text { thick }\end{array}$} & \multirow[b]{2}{*}{ Type } & \multirow[b]{2}{*}{ Hf } & \multirow[b]{2}{*}{ C } & \multicolumn{10}{|c|}{ Chemical Composition (Nominal Weight Percent) } \\
\hline & & & & $\mathrm{Cr}$ & $\mathrm{Cu}$ & $\mathrm{Fe}$ & Mn & Mo & $\mathbf{N i}$ & $\mathbf{N}$ & $\mathrm{Si}$ & $\mathrm{Ti}$ & $\mathbf{W}$ \\
\hline 1234 & $\begin{array}{l}\text { Unalloyed } \\
\text { Zirconium A }\end{array}$ & 46 & $(80)$ & $(55)$ & $(<10)$ & $(240)$ & (10) & $(2)$ & (20) & (14) & $(40)$ & $(20)$ & $(25)$ \\
\hline 1235 & $\begin{array}{l}\text { Unalloyed } \\
\text { Zirconium B }\end{array}$ & 95 & $(170)$ & $(60)$ & $(80)$ & $(850)$ & (25) & (40) & (65) & (32) & (95) & $(90)$ & $(50)$ \\
\hline 1236 & $\begin{array}{l}\text { Unalloyed } \\
\text { Zirconium C }\end{array}$ & 198 & (280) & (250) & (250) & (1700) & (45) & (100) & (140) & $(69)$ & (205) & $(185)$ & $(140)$ \\
\hline 1237 & Zircaloy D & 31 & $(100)$ & $(1510)$ & $(<10)$ & $(1650)$ & $(10)$ & $(<10)$ & $(40)$ & $(19)$ & $(35)$ & $(30)$ & $(25)$ \\
\hline 1238 & Zircalloy E & 178 & (310) & (580) & (60) & $(2500)$ & (60) & (120) & $(100)$ & $(72)$ & (170) & $(100)$ & $(95)$ \\
\hline 1239 & Zircalloy F & 77 & (170) & (1055) & $(130)$ & $(2300)$ & (50) & (45) & (45) & (42) & (95) & $(40)$ & $(45)$ \\
\hline
\end{tabular}

Values in parentheses are not certified, but are given for information only.

\section{Gases in Metals}

\section{Certified for Hydrogen, Oxygen, and Nitrogen}

The SRM's are used in the determination of hydrogen, oxygen and nitrogen by vacuum fusion, inert gas fusion, and neutron activation methods. SRM's 1095 to 1099 were prepared from the same melt as the "1200" series, (1261a-1265a), see page 26.

\begin{tabular}{|c|c|c|c|c|c|}
\hline SRM & Type & Form & $\begin{array}{c}\text { Oxygen } \\
(\mathrm{ppm})\end{array}$ & $\begin{array}{l}\text { Hydrogen } \\
\quad(\mathrm{ppm})\end{array}$ & $\begin{array}{c}\text { Nitrogen } \\
(\mathrm{ppm})\end{array}$ \\
\hline $352 \mathrm{~b}$ & Unalloyed titanium for hydrogen & Platelets & & 50 & \\
\hline 354 & Unalloyed titanium for hydrogen & IN PREP & & & \\
\hline$\overline{355}$ & Unalloyed titanium & Rod & 3031 & & \\
\hline 357 & Unalloyed Zirconium & Wire & $(1200)$ & 19 & 49 \\
\hline 358 & Unalloyed Zirconium & Wire & $(1100)$ & 107 & 28 \\
\hline 1086 & Unalloyed Titanium & Chips & $(1350)$ & 116 & \\
\hline 1087 & Unalloyed Titanium & Chips & $(840)$ & 57.5 & \\
\hline 1088 & Unalloyed Titanium & Chips & $(1450)$ & 88.5 & \\
\hline 1090 & Ingot iron & Rod & 491 & & $(60)$ \\
\hline 1091 & Stainless steel (AISI 431) & Rod & 131 & & $(945)$ \\
\hline 1092 & Vacuum-melted steel & Rod & 28 & & (4) \\
\hline 1093 & Valve steel & Rod & 60 & & $(4807)$ \\
\hline 1094 & Maraging steel & Rod & 4.5 & & (71) \\
\hline${ }^{*} 1095$ & AISI 4340 steel & Rod & 9 & & (37) \\
\hline${ }^{* 1096}$ & AISI 94B17 (Mod)steel & Rod & 10.7 & & 40.4 \\
\hline$\overline{* 1097}$ & Cr-V (Mod)steel & Rod & 6.6 & & $(41)$ \\
\hline *1098 & High Carbon (Mod)steel & Rod & 10 & & 32 \\
\hline$* 1099$ & Electrolytic iron & Rod & 61 & & $\overline{(13)}$ \\
\hline 1089 & Set of $5: 1095,1096,1097,1098$, and 1099 & Rods & & & \\
\hline
\end{tabular}

*Sold in sets as SRM 1089.

Values in parentheses are not certified, but are given for information only. 
High-purity metal SRM's are available for purposes of determining impurity elements in high-purity metal materials. They are intended to serve as benchmark standards in the calibration of methods and equipment. They are also expected to be valuable to the development of new or improved methods and techniques for increasing the sensitivity of detection for trace elements in various materials.

The Certificate of Analysis supplied with each SRM gives information on its chemical composition and the values obtained by cooperating laboratories.

High-purity gold is available in both wire and rod form. The wire form (W), is intended for applications such as spark source mass spectroscopic techniques. The low levels of impurities make it valuable in evaluating instrument and system blanks. The rod form $(\mathrm{R})$, is intended for application in other methods of characterization.

Platinum is available in wire form both as a high-purity material and as doped composition material.

Zinc is available in a high-purity and in a less pure version. Both were prepared from the same starting material. The high-purity material is the result of further purification by vacuum distillation, zone refining, and degasification. The zinc is supplied in the form of semi-circular bar segments.

SRM 769 is a set of five aluminum rods that have residual resistivity ratios, $\rho(273 \mathrm{~K}) / \rho(4 \mathrm{~K})$, ranging from 130 to 11,000 . This ratio can be a sensitive indicator of the purity of metals.

\begin{tabular}{|c|c|c|c|c|c|c|c|}
\hline \multirow[b]{2}{*}{ SRM } & \multirow[b]{2}{*}{ Type } & \multirow[b]{2}{*}{ Unit Size } & \multicolumn{5}{|c|}{$\begin{array}{c}\text { Chemical Compositions (Nominal Parts } \\
\text { Per Million by Weight) }\end{array}$} \\
\hline & & & $\mathrm{Cu}$ & Ni & Sn & $\mathbf{P b}$ & $\mathrm{Sr}$ \\
\hline $685 W^{*}$ & High-Purity Gold (Wire) & $1.4 \mathrm{~mm} \mathrm{D} \times 102 \mathrm{~mm}$ long & 0.01 & & & & \\
\hline $685 \mathrm{R}^{*}$ & High-Purity Gold (Rod) & $5.9 \mathrm{~mm} \mathrm{D} \times 25 \mathrm{~mm}$ long & 0.1 & & & & \\
\hline $680 \mathrm{aLl}$ & High-Purity Platinum (Wire) & $0.51 \mathrm{~mm} \mathrm{D} \times 102 \mathrm{~mm}$ long & 0.1 & $<1$ & & $<1$ & $\langle 0.1$ \\
\hline $680 \mathrm{aL} 2$ & High-Purity Platinum (Wire) & $0.51 \mathrm{~mm} \mathrm{D} \times 1.0 \mathrm{~m}$ long & 0.1 & $<1$ & & $<1$ & $\langle 0.1$ \\
\hline $681 \mathrm{L1}$ & Doped-Platinum (Wire) & $0.51 \mathrm{~mm} \mathrm{D} \times 102 \mathrm{~mm}$ long & 5.1 & 0.5 & & 12 & 11 \\
\hline $681 \mathrm{~L} 2$ & Doped-Platinum (Wire) & $0.51 \mathrm{~mm} \mathrm{D} \times 1.0 \mathrm{~m}$ long & 5.1 & 0.5 & & 12 & 11 \\
\hline $682^{*}$ & High-Purity Zine & $\begin{array}{l}\text { Semicircular segments } 57 \mathrm{~mm} \mathrm{D} \\
\times 19 \mathrm{~mm} \text { long } \\
\end{array}$ & 0.042 & & $(0.02)$ & & \\
\hline $683^{*}$ & Zinc Metal & Semicircular segments $57 \mathrm{~mm} \mathrm{D}$ & 5.9 & & $(0.02)$ & 11.1 & \\
\hline 769 & $\begin{array}{l}\text { Electrical Residual } \\
\text { Resistivity Ratio }\end{array}$ & $6.4 \mathrm{~mm} \mathrm{D} \times 55 \mathrm{~mm}$ long & & & & & \\
\hline
\end{tabular}

\begin{tabular}{|c|c|c|c|c|c|c|c|c|c|c|c|}
\hline SRM & $\mathrm{Ag}$ & $\mathbf{M g}$ & In & $\mathrm{Fe}$ & $\mathrm{O}$ & Pd & $\mathrm{Au}$ & $\mathbf{R h}$ & Ir & Cd & $\mathrm{Ti}$ \\
\hline $685 W^{*}$ & {$[0.1]$} & & 0.007 & 0.3 & [2] & & & & & & \\
\hline $685 R^{*}$ & {$[0.1]$} & & 0.007 & 0.2 & $\mid<2]$ & & & & & & \\
\hline $680 \mathrm{aLl}$ & $<0.1$ & $<1$ & & 1.3 & 4 & 0.2 & $<1$ & $<0.2$ & $<0.01$ & & \\
\hline $680 \mathrm{aL} 2$ & $<0.1$ & $<1$ & & 1.3 & 4 & 0.2 & $<1$ & $<0.2$ & 0.01 & & \\
\hline $681 \mathrm{Ll}$ & 2.0 & 12 & & 5 & 7 & 6 & 9 & 9 & 11 & & \\
\hline$\overline{681 \mathrm{~L} 2}$ & 2.0 & 12 & & 5 & 7 & 6 & 9 & 9 & 11 & & \\
\hline $682^{*}$ & $(0.02)$ & & & $(0.1)$ & & & & & & $(0.1)$ & \\
\hline $683^{*}$ & 1.3 & & & 2.2 & & & & & & 1.1 & $(0.2)$ \\
\hline
\end{tabular}

*Certificate gives upper limits for other elements found to be present.

Values in parentheses are not certified, but are given for information only.

Values in brackets are subject to greater error since only one meihod of analysis was employed. 
These SRM's provide a highly homogeneous material at microscopic spatial resolution. They are intended primarily for use in calibration of quantitative electron probe, secondary ion mass spectrometry, spark sources, mass spectrometry, and laser probe microanalytical techniques.

\section{Mineral Glasses for Microanalysis}

SRM 470 consists of two mineral glasses that have the following approximate compositions. Details of the measurements on these glasses are in NBS Special Publication 260-74.

\begin{tabular}{|lccccc|}
\hline & & Composition (Nominal & Weight Percent) & \\
\hline $\mathrm{Glass}$ & $\mathrm{SiO}_{2}$ & $\mathrm{FeO}$ & $\mathrm{MgO}$ & $\mathrm{CaO}$ & 15 \\
\hline $\mathrm{K}-411$ & 55 & 15 & 15 & $\mathrm{Al}_{2} \mathrm{O}_{3}$ \\
\hline $\mathrm{K}-412$ & 45 & 10 & 20 & 15 & 10 \\
\hline
\end{tabular}

\section{Glasses for Microchemical Analysis}

Each SRM, 1871 through 1875, consists of three glasses, two with and one without low-concentration constituents. The major constituents of the glasses indicated below were selected so that the average atomic numbers would be different.

\begin{tabular}{|c|c|c|c|c|c|}
\hline SRM & 1871 & 1872 & 1873 & 1874 & 1875 \\
\hline \multicolumn{6}{|c|}{$\begin{array}{c}\text { Composition } \\
\text { (Nominal Weight Percent) }\end{array}$} \\
\hline $\mathrm{PbO}$ & 71 & 59 & & & \\
\hline $\mathrm{SiO}_{2}$ & 29 & & 49 & & \\
\hline $\mathrm{GeO}_{2}$ & & 41 & & & \\
\hline $\mathrm{BaO}$ & & & 47 & & \\
\hline $\mathrm{ZnO}$ & & & 4 & & \\
\hline $\mathrm{B}_{2} \mathrm{O}_{3}$ & & & & 75 & \\
\hline $\mathrm{AI}_{2} \mathrm{O}_{3}$ & & & & 20 & \\
\hline $\mathrm{Li}_{2} \mathrm{O}$ & & & & 5 & \\
\hline $\mathrm{P}_{2} \mathrm{O}_{5}$ & & & & & 80 \\
\hline$\overline{\mathrm{MgO}}$ & & & & & 9 \\
\hline
\end{tabular}

\section{Cartridge Brass}

Cartridge Brass, SRM 478, consists of two specimens: a chill-cast cube with a polished chill-cast face and a wrought right circular cylinder. Both the cube and the cylinder are homogeneous at micrometer levels of spatial resolution for both copper and zinc. Details of the homogeneity testing are in NBS Special Publications 260-10 and 260-65.

\section{Fe-Cr-Ni Alloy}

The Fe-Cr-Ni alloy, SRM 479a, is a wafer characterized for chemical homogeneity of iron, chromium, and nickel at the micrometer level of spatial resolution. It is satisfactory for use as a homogeneous material for electron probe microanalysis. See NBS Special Publication 260-70 for details of the testing. 


\section{Tungsten-20\% Molybdenum}

The tungsten-20\% molybdenum alloy, SRM 480, is a wafer with a core of tungsten-20\% molybdenum wire embedded in pure molybdenum onto which pure tungsten has been deposited by electroplating to provide a composite structure. Details on homogeneity characterization are given in NBS Special Publications 260-16 and 260-65.

\section{Gold-Silver}

Six color-coded wires comprise SRM 481. The wires consist of a high-purity gold and a high-purity silver wire and four wires with nominal chemical composition differences in steps of 20 percent. See NBS Special Publications 260-28 and 260-65.

\section{Gold-Copper}

Six color-coded wires comprise SRM 482, which is similar to the gold-silver set. In both sets special precautions were taken to achieve homogeneity on a microscopic scale. See NBS Special Publications 260-28 and $260-65$.

\section{Iron-3.22\% Sillicon}

The iron-3.22\% silicon alloy SRM 483, is a platelet characterized for chemical homogeneity of iron and silicon at the micrometer level of spatial resoltion. It is satisfactory for use as a homogeneous material for electron probe microanalysis. See NBS Special Publications 260-22 and 260-65.

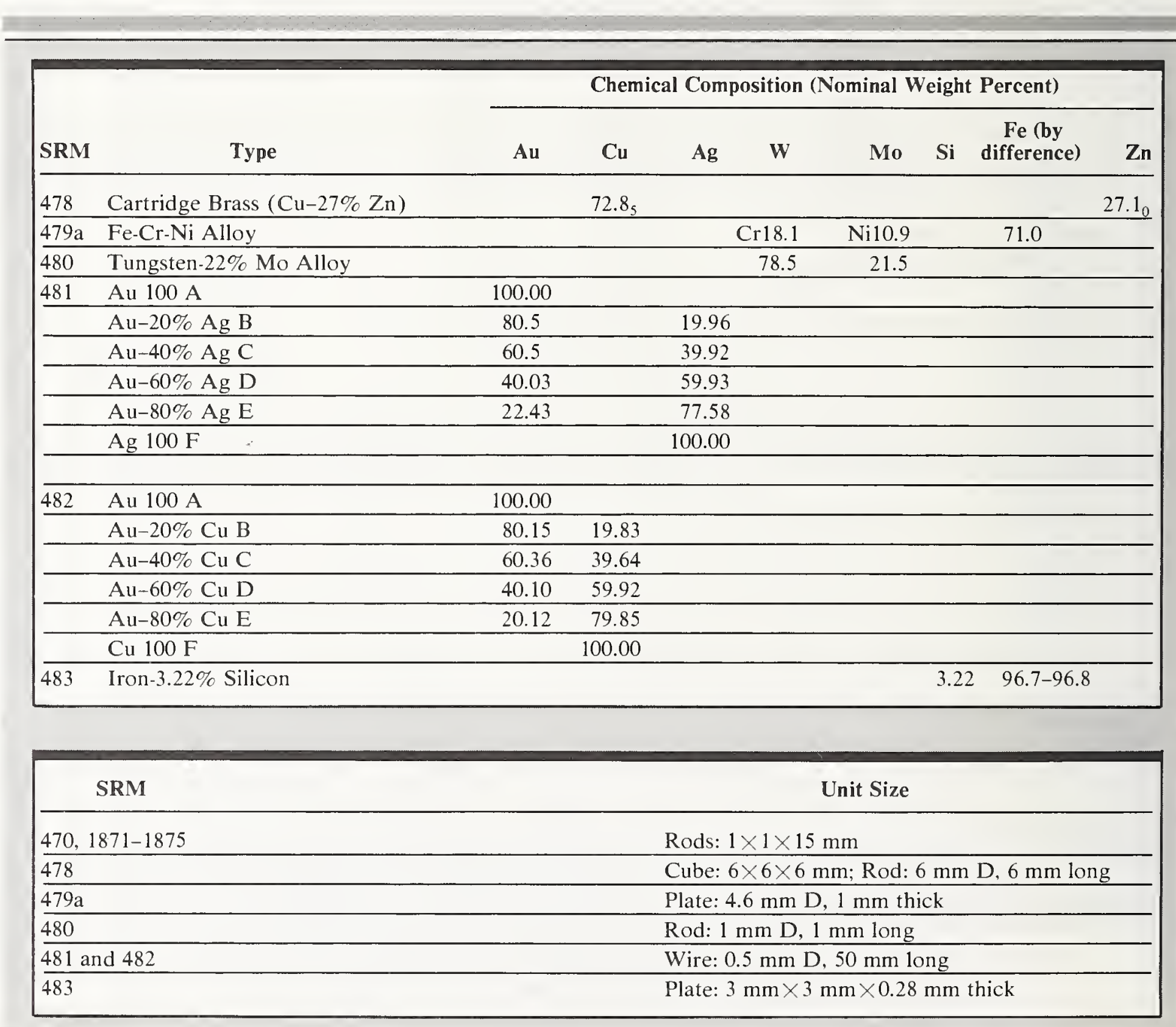




\section{Primary, Working, and Secondary Chemicalls}

These SRM's are high-purity chemicals defined as primary, working, and secondary standards in accordance with recommendations of the Analytical Chemistry Section of the International Union of Pure and Applied Chemistry [Ref. Analyst 90, 251 (1965)]. These definitions are as follows:

Primary Standard:

a commercially available substance of purity $100 \pm 0.02$ percent (Purity $99.98+$ percent).

Working Standard:

a commercially available substance of purity $100 \pm 0.05$ percent (Purity $99.95+$ percent).

Secondary Standard:

a substance of lower purity which can be standardized against a primary grade standard.

\begin{tabular}{|c|c|c|c|c|}
\hline SRM & Type & $\begin{array}{l}\text { Wt/Unit } \\
\text { (grams) }\end{array}$ & Certified Use & $\begin{array}{c}\text { Purity } \\
\text { Stoichiometric }\end{array}$ \\
\hline $17 \mathrm{c}$ & Sucrose & 60 & Polarimetric Value & $\left({ }^{a}\right)$ \\
\hline $40 \mathrm{~h}$ & Sodium Oxalate & 60 & Reductometric Value & 99.972 \\
\hline $41 \mathrm{~b}$ & Dextrose (D-glucose) & 70 & Reductometric Value & $\left({ }^{b}\right)$ \\
\hline $83 \mathrm{~d}$ & Arsenic Trioxide & 60 & Reductometric Value & 99.9926 \\
\hline $84 \mathrm{j}$ & Acid Potassium Phthalate & 60 & Acidimetric Value & 99.996 \\
\hline $136 \mathrm{~d}$ & Potassium Dichromate & 60 & Oxidimetric Value & 99.9931 \\
\hline$\overline{350 a}$ & Benzoic Acid & 30 & Acidimetric Value & 99.9958 \\
\hline $723 a$ & Tris(hydroxymethyl)aminomethane & 50 & Basimetric Value & 99.9703 \\
\hline $949 \mathrm{f}$ & Plutonium Metal & 0.5 & Assay & 99.99 \\
\hline $950 \mathrm{~b}$ & Uranium Oxide $\left(\mathrm{U}_{3} \mathrm{O}_{8}\right)$ & 25 & Uranium Oxide Standard Value & 99.968 \\
\hline 951 & Boric Acid & 100 & Acidimetric and Boron Isotopic Value & 100.00 \\
\hline 960 & Uranium Metal & 26 & Assay & 99.975 \\
\hline 985 & Potassium Chloride & 1 & Assay and Isotopic & 99.9 \\
\hline 987 & Strontium Carbonate & 1 & Assay and Isotopic & 99.98 \\
\hline \multirow[t]{3}{*}{999} & Potassium Chloride & 60 & Assay Standard for: & \\
\hline & & & Potassium & 99.98 \\
\hline & & & Chloride & 99.99 \\
\hline
\end{tabular}

${ }^{\text {a }}$ Sucrose $=$ Moisture $<0.01$ percent, Reducing Substance $<0.02$ percent, Ash 0.001 percent.

${ }^{\mathrm{b}}$ Dextrose $=$ Moisture 0.07 percent, Ash 0.002 percent.

\section{Microchemicall}

These SRM's are furnished as fine crystals of suitable homogeneity for use as standards for conventional microchemical methods of analysis employing samples of approximately $5 \mathrm{mg}$. See also Microanalytical Standards, page 53 .

\begin{tabular}{|c|c|c|c|}
\hline SRM & Type & Wt/Unit (grams) & Elements Certified \\
\hline $140 \mathrm{~b}$ & Benzoic Acid & 2 & $\mathrm{C}, \mathrm{H}$ (IN PREP) \\
\hline $141 \mathrm{c}$ & Acetanilide & 2 & $\mathrm{~N}, \mathrm{C}, \mathrm{H}$ \\
\hline 142 & Anisic Acid & 2 & Methoxyl $\left(\mathrm{CH}_{3} \mathrm{O}-\right)$ \\
\hline $143 c$ & Cystine & 2 & $\mathrm{~S}, \mathrm{C}, \mathrm{H}, \mathrm{N}$ \\
\hline 148 & Nicotinic Acid & 2 & $\mathrm{~N}, \mathrm{C}, \mathrm{H}$ \\
\hline
\end{tabular}




\section{(Continued)}

\begin{tabular}{|c|c|c|c|}
\hline 2141 & Urea & 2 & $\mathrm{~N}$ \\
\hline 2142 & o-Bromobenzoic Acid & 2 & $\mathrm{Br}$ \\
\hline 2143 & p-Fluorobenzoic Acid & 2 & $\bar{F}$ \\
\hline 2144 & m-Chlorobenzoic Acid & 2 & $\mathrm{Cl}$ \\
\hline
\end{tabular}

\section{Cilinicall Laboratory}

These SRM's are intended for use in calibrating apparatus and validating analytical methods used in clinical and pathological laboratories, and to assist manufacturers of clinical products in meeting the chemical and physical specifications required for clinical chemicals. (For details on SRM's 930D and 931c, see Spectrophotometric Filters, page 96.)

\begin{tabular}{|c|c|c|c|c|}
\hline SRM & Туре & $\begin{array}{c}\text { Associated } \\
\text { NBS Publications }\end{array}$ & Purity \% & Wt/Unit \\
\hline 900 & $\begin{array}{l}\text { Antiepilepsy Drug Level Assay (phenytoin, } \\
\text { ethosuximide, phenobarbital, and primidone }\end{array}$ & & 4 drugs/3 levels & Set of 4 vials \\
\hline 909 & Human Serum & & \# & Set of 6 vials \\
\hline 910 & Sodium Pyruvate & & 98.7 & $25 \mathrm{~g}$ \\
\hline$\underline{911 \mathrm{a}}$ & Cholesterol & & 99.8 & $2 \mathrm{~g}$ \\
\hline $912 \mathrm{a}$ & Urea & & 99.9 & $25 \mathrm{~g}$ \\
\hline 913 & Uric Acid & & 99.7 & $10 \mathrm{~g}$ \\
\hline 914 & Creatinine & & 99.8 & $10 \mathrm{~g}$ \\
\hline 915 & Calcium Carbonate & SP 260-36 & 99.9 & $20 \mathrm{~g}$ \\
\hline$\underline{916}$ & Bilirubin & & 99.0 & $100 \mathrm{mg}$ \\
\hline 917 & D.Glucose & & 99.9 & $25 \mathrm{~g}$ \\
\hline 918 & Potassium Chloride & SP 260-63 & 99.9 & $30 \mathrm{~g}$ \\
\hline 919 & Sodium Chloride & SP 260-60 & 99.9 & $30 \mathrm{~g}$ \\
\hline 920 & D-Mannitol & & 99.8 & $50 \mathrm{~g}$ \\
\hline 921 & Cortisol & & 98.9 & $1 \mathrm{~g}$ \\
\hline$\overline{922}$ & Tris(hydroxymethyl) aminomethane & & 99.9 & $25 \mathrm{~g}$ \\
\hline 923 & Tris(hydroxymethyl) aminomethane $\mathrm{HCl}$ & & 99.7 & $35 \mathrm{~g}$ \\
\hline$\underline{924}$ & Lithium Carbonate & SP $260-70$ & 100.0 & $30 \mathrm{~g}$ \\
\hline 925 & VMA (4-hydroxy-3-methodymandelic acid) & & 99.4 & $1 \mathrm{~g}$ \\
\hline$\underline{926}$ & Bovine Serum Albumin (Powder) & & $* *$ & $5 \mathrm{~g}$ \\
\hline 927 & Bovine Serum Albumin ( $7 \%$ Solution) & & $* *$ & 10 vials, $2.15 \mathrm{~mL}$ ea. \\
\hline$\underline{928}$ & Lead Nitrate & & 100.00 & $30 \mathrm{~g}$ \\
\hline$\underline{929}$ & Magnesium Gluconate & & 100.1 & $5 \mathrm{~g}$ \\
\hline $930 \mathrm{D}$ & Glass Filters for Spectrophotometry & SP 260-51 & + & Set of 3 \\
\hline $931 \mathrm{c}$ & Liquid Filters for Spectrophotometry & & + & Set of 12 vials \\
\hline
\end{tabular}




\section{(Continued)}

\begin{tabular}{|c|c|c|c|c|}
\hline SRM & Type & $\begin{array}{c}\text { Associated } \\
\text { NBS Publications }\end{array}$ & Purity \% & Wt/Unit \\
\hline 932 & Quartz Cuvette for Spectrophotometry & SP $260-32$ & + & 1 each \\
\hline 934 & Clinical Laboratory Thermometer & SP $260-48$ & Ht & 1 each \\
\hline 935 & $\begin{array}{l}\text { Crystalline Potassium Dichromate } \\
\text { (UV Absorbance)Standard }\end{array}$ & SP $260-54$ & $(99.972)^{* * *}$ & $15 \mathrm{~g}$ \\
\hline 936 & Quinine Sulfate Dihydrate (Fluorescence) & SP 260-64 & $(98.2)^{* * *}$ & $1 \mathrm{~g}$ \\
\hline 937 & Iron Metal & & 99.90 & $50 \mathrm{~g}$ \\
\hline$\overline{938}$ & 4-Nitrophenol, Clinical & & 99.75 & $15 \mathrm{~g}$ \\
\hline 998 & Angiotensin I (Human) & & 94.1 & $500 \mu \mathrm{g}$ \\
\hline 1590 & Stabilized Wine & & $\begin{array}{l}18.57 \% \\
\text { by volume } \\
\text { at } 20{ }^{\circ} \mathrm{C} \\
\end{array}$ & Set of 10 vials \\
\hline 1595 & Tripalmitin & & 99.5 & $2 \mathrm{~g}$ \\
\hline$\overline{1599}$ & $\begin{array}{l}\text { Anticonvulsant Drug Level Assay } \\
\text { (valproic acid and carbamazepine) }\end{array}$ & & $\begin{array}{l}2 \text { drugs/ } \\
3 \text { levels }\end{array}$ & Set of 4 vials \\
\hline 1968 & Gallium Melting Point $29.7723{ }^{\circ} \mathrm{C}$ & SP 481 & & 1 each \\
\hline 1969 & Rubidium Melting Point $38^{\circ} \mathrm{C}$ & & IN PREP & 1 each \\
\hline 1970 & Succinonitrile Freezing Point $58{ }^{\circ} \mathrm{C}$ & & IN PREP & 1 each \\
\hline
\end{tabular}

+ Certified for optical properties (see p. 00).

H Individually calibrated at $0,25,30$ and $37^{\circ} \mathrm{C}$. For further information regarding calibration services, call 301-921-2805.

** Conforms to NCCLS specification ACC-1.

*** Apparent purity, certified for optical properties.

\# Electrolytes, selected organics.

These SRM's are intended for use in the calibration of apparatus and methods used in the analysis of biological materials for major, minor, and trace constituents.

\begin{tabular}{|c|c|c|c|c|c|c|c|c|c|}
\hline SRM & 1549 & 1566 & 1567 & 1568 & 1569 & 1572 & 1573 & 1575 & $1577 a$ \\
\hline Type & $\begin{array}{l}\text { Non-fat } \\
\text { Powdered } \\
\text { Milk }\end{array}$ & $\begin{array}{l}\text { Oyster } \\
\text { Tissue }\end{array}$ & $\begin{array}{l}\text { Wheat } \\
\text { Flour }\end{array}$ & $\begin{array}{l}\text { Rice } \\
\text { Flour }\end{array}$ & $\begin{array}{l}\text { Brewers } \\
\text { Yeast }\end{array}$ & $\begin{array}{l}\text { Citrus } \\
\text { Leaves }\end{array}$ & $\begin{array}{l}\text { Tomato } \\
\text { Leaves }\end{array}$ & $\begin{array}{l}\text { Pine } \\
\text { Needles }\end{array}$ & $\begin{array}{l}\text { Bovine } \\
\text { Liver }\end{array}$ \\
\hline Unit Size & IN PREP & $30 \mathrm{~g}$ & $80 \mathrm{~g}$ & $80 \mathrm{~g}$ & $50 \mathrm{~g}$ & $70 \mathrm{~g}$ & $70 \mathrm{~g}$ & $70 \mathrm{~g}$ & $50 \mathrm{~g}$ \\
\hline \multicolumn{10}{|l|}{ ELEMENT } \\
\hline Aluminum & & & & & & 92 & $(0.12 \%)$ & 545 & $(2)$ \\
\hline Antimony & & & & & & $(0.04)$ & & $(0.2)$ & $(0.003)$ \\
\hline Arsenic & & 13.4 & $(0.006)$ & 0.41 & & 3.1 & 0.27 & 0.21 & 0.047 \\
\hline Barium & & & & & & 21 & & & \\
\hline \multicolumn{10}{|l|}{ Beryllium } \\
\hline \multicolumn{10}{|l|}{ Bismuth } \\
\hline Boron & & & & & & & $(30)$ & & \\
\hline Bromine & & $(55)$ & $(9)$ & (1) & & $(8.2)$ & $(26)$ & (9) & (9) \\
\hline Cadmium & & 3.5 & 0.032 & 0.029 & & 0.03 & $(3)$ & $(<0.5)$ & 0.44 \\
\hline Calcium & & $0.15 \%$ & $0.019 \%$ & $0.014 \%$ & & $3.15 \%$ & $3.00 \%$ & $0.41 \%$ & 120 \\
\hline
\end{tabular}




\section{(Continued)}

\begin{tabular}{|c|c|c|c|c|c|c|c|c|c|}
\hline SRM & 1549 & 1566 & 1567 & 1568 & 1569 & 1572 & 1573 & 1575 & $1577 a$ \\
\hline Type & $\begin{array}{l}\text { Non-fat } \\
\text { Powdered } \\
\text { Milk }\end{array}$ & $\begin{array}{l}\text { Oyster } \\
\text { Tissue }\end{array}$ & $\begin{array}{l}\text { Wheat } \\
\text { Flour }\end{array}$ & $\begin{array}{l}\text { Rice } \\
\text { Flour }\end{array}$ & $\begin{array}{l}\text { Brewers } \\
\text { Yeast }\end{array}$ & $\begin{array}{l}\text { Citrus } \\
\text { Leaves }\end{array}$ & $\begin{array}{l}\text { Tomato } \\
\text { Leaves }\end{array}$ & $\begin{array}{l}\text { Pine } \\
\text { Needles }\end{array}$ & $\begin{array}{l}\text { Bovine } \\
\text { Liver }\end{array}$ \\
\hline Unit Size & IN PREP & $30 \mathrm{~g}$ & $80 \mathrm{~g}$ & $80 \mathrm{~g}$ & $50 \mathrm{~g}$ & $70 \mathrm{~g}$ & $70 \mathrm{~g}$ & $70 \mathrm{~g}$ & $50 \mathrm{~g}$ \\
\hline Cerium & & & & & & $(0.28)$ & $(1.6)$ & $(0.4)$ & \\
\hline Cesium & & & & & & $(0.098)$ & & & \\
\hline Chlorine & & $(1.0 \%)$ & & & & (414) & & & $0.28 \%$ \\
\hline Chromium & & 0.69 & & & 2.12 & 0.8 & 4.5 & 2.6 & \\
\hline Cobalt & & $(0.4)$ & & 0.02 & & $(0.02)$ & $(0.6)$ & $(0.1)$ & 0.21 \\
\hline Copper & & 63.0 & 2.0 & 2.2 & & 16.5 & 11 & 3.0 & 158 \\
\hline Europium & & & & & & $(0.01)$ & $(0.04)$ & $(0.006)$ & \\
\hline Fluorine & & $(5.2)$ & & & & & & & \\
\hline \multicolumn{10}{|l|}{ Gallium } \\
\hline Iodine & & $(2.8)$ & & & & 1.84 & & & \\
\hline Iron & & 195 & 18.3 & 8.7 & & 90 & 690 & 200 & 194 \\
\hline Lathanum & & & & & & $(0.19)$ & $(0.9)$ & $(0.2)$ & \\
\hline Lead & & 0.48 & 0.020 & 0.045 & & 13.3 & 6.3 & 10.8 & 0.135 \\
\hline \multicolumn{10}{|l|}{ Lithium } \\
\hline Magnesium & & $0.128 \%$ & & & & $0.58 \%$ & $(0.7 \%)$ & & 600 \\
\hline Manganese & & 17.5 & 8.5 & 20.1 & & 23 & 238 & 675 & 9.9 \\
\hline Mercury & & 0.057 & 0.001 & 0.0060 & & 0.08 & $(0.1)$ & 0.15 & 0.004 \\
\hline Molybdenum & & $(\leqslant 0.2)$ & $(0.4)$ & $(1.6)$ & & 0.17 & & & 3.5 \\
\hline Nickel & & 1.3 & $(0.18)$ & $(0.16)$ & & 0.6 & & $(3.5)$ & \\
\hline Nitrogen & & & & & & $(2.86 \%)$ & $(5.0 \%)$ & $(1.2 \%)$ & $(10.7 \%)$ \\
\hline Phosphorus & & $(0.81 \%)$ & & & & $0.13 \%$ & $0.34 \%$ & $0.12 \%$ & $1.11 \%$ \\
\hline Potassium & & $0.969 \%$ & $0.136 \%$ & $0.112 \%$ & & $1.82 \%$ & $4.46 \%$ & $0.37 \%$ & $0.996 \%$ \\
\hline Rubidium & & 4.45 & (1) & $(7)$ & & 4.84 & 16.5 & 11.7 & 12.5 \\
\hline Samarium & & & & & & $(0.052)$ & & & \\
\hline Scandium & & & & & & $(0.01)$ & $(0.13)$ & $(0.03)$ & \\
\hline Selenium & & 2.1 & 1.1 & 0.4 & & $(0.025)$ & & & 0.71 \\
\hline Silver & & 0.89 & & & & & & & 0.04 \\
\hline Sodium & & $0.51 \%$ & 8.0 & 6.0 & & 160 & & & $0.243 \%$ \\
\hline Strontium & & 10.36 & & & & 100 & 44.9 & 4.8 & 0.138 \\
\hline Sulfur & & $(0.76 \%)$ & & & & $0.407 \%$ & & & $0.78 \%$ \\
\hline Tellurium & & & $(\leqslant 0.002)$ & $(\leqslant 0.002)$ & & $(0.02)$ & & & \\
\hline Thallium & & $(\leqslant 0.005)$ & & & & $(\leqslant 0.01)$ & $(0.05)$ & $(0.05)$ & $(0.003)$ \\
\hline Thorium & & $(0.1)$ & & & & & 0.17 & 0.037 & \\
\hline Tin & & & & & & $(0.24)$ & & & \\
\hline Uranium & & 0.116 & & & & $(\leqslant 0.15)$ & 0.061 & 0.020 & 0.00071 \\
\hline Vanadium & & 2.3 & & & & & & & \\
\hline$\overline{\text { Zinc }}$ & & 852 & 10.6 & 19.4 & & 29 & 62 & & 123 \\
\hline
\end{tabular}

Content in $\mu \mathrm{g} / \mathrm{g}$, or where noted in weight percent.

Values in parentheses are not certified, but are given for information on 


\section{Analyzed Gases}

These SRM's are intended for use in the calibration of apparatus used for the measurement of various components in gas mixtures, and in some cases for particular atmospheric pollutants. Each SRM in accurately certified and is intended primarily for use in monitoring and correcting long-term drifts in instruments used. Each gas is contained in an aluminum cylinder, 870 liters at STP. All cylinders conform to the appropriate DOT specifications.

\begin{tabular}{|c|c|c|c|c|}
\hline SRM & Type & $\begin{array}{c}\text { Certified } \\
\text { Component }\end{array}$ & & Concentration \\
\hline $1658 \mathrm{a}$ & Methane in Air & $\mathrm{CH}_{4}$ & 1 & $\mu$ mole $/$ mole $(\mathrm{ppm})$ \\
\hline $1659 \mathrm{a}$ & Methane in Air & $\mathrm{CH}_{4}$ & 10 & $\mu$ mole $/$ mole $(\mathrm{ppm})$ \\
\hline \multirow[t]{2}{*}{$1660 \mathrm{a}$} & Methane-Propane in Air & $\mathrm{CH}_{4}$ & 4 & $\mu$ mole $/$ mole $(\mathrm{ppm})$ \\
\hline & & $\mathrm{C}_{3} \mathrm{H}_{8}$ & 1 & $\mu \mathrm{mole} / \mathrm{mole}(\mathrm{ppm})$ \\
\hline $1661 \mathrm{a}$ & Sulfur Dioxide in Nitrogen & $\mathrm{SO}_{2}$ & 500 & $\mu$ mole $/$ mole $(\mathrm{ppm})$ \\
\hline $1662 \mathrm{a}$ & Sulfur Dioxide in Nitrogen & $\mathrm{SO}_{2}$ & 1000 & $\mu$ mole $/$ mole $(\mathrm{ppm})$ \\
\hline $1663 \mathrm{a}$ & Sulfur Dioxide in Nitrogen & $\mathrm{SO}_{2}$ & 1500 & $\mu \mathrm{mole} / \mathrm{mole}(\mathrm{ppm})$ \\
\hline $1664 a$ & Sulfur Dioxide in Nitrogen & $\mathrm{SO}_{2}$ & 2500 & $\mu$ mole $/$ mole $(\mathrm{ppm})$ \\
\hline $1665 b$ & Propane in Air & $\mathrm{C}_{3} \mathrm{H}_{8}$ & 3 & $\mu$ mole $/$ mole $(\mathrm{ppm})$ \\
\hline $1666 \mathrm{~b}$ & Propane in Air & $\mathrm{C}_{3} \mathrm{H}_{8}$ & 10 & $\mu \mathrm{mole} / \mathrm{mole}(\mathrm{ppm})$ \\
\hline $1667 \mathrm{~b}$ & Propane in Air & $\mathrm{C}_{3} \mathrm{H}_{8}$ & 50 & $\mu$ mole $/$ mole $(\mathrm{ppm})$ \\
\hline $1668 \mathrm{~b}$ & Propane in Air & $\mathrm{C}_{3} \mathrm{H}_{8}$ & 100 & $\mu$ mole $/$ mole $(\mathrm{ppm})$ \\
\hline $1669 \mathrm{~b}$ & Propane in Air & $\mathrm{C}_{3} \mathrm{H}_{8}$ & 500 & $\mu$ mole $/$ mole $(\mathrm{ppm})$ \\
\hline 1670 & Carbon Dioxide in Air & $\mathrm{CO}_{2}$ & 0.033 & mole percent \\
\hline 1671 & Carbon Dioxide in Air & $\mathrm{CO}_{2}$ & 0.034 & mole percent \\
\hline 1672 & Carbon Dioxide in Air & $\mathrm{CO}_{2}$ & 0.035 & mole percent \\
\hline $1674 \mathrm{~b}$ & Carbon Dioxide in Nitrogen & $\mathrm{CO}_{2}$ & 7.0 & mole percent \\
\hline $1675 b$ & Carbon Dioxide in Nitrogen & $\mathrm{CO}_{2}$ & 14.0 & mole percent \\
\hline $1677 \mathrm{c}$ & Carbon Monoxide in Nitrogen & $\mathrm{CO}$ & 10 & $\mathrm{ppm}$ \\
\hline $1678 \mathrm{c}$ & Carbon Monoxide in Nitrogen & $\mathrm{CO}$ & 50 & $\mathrm{ppm}$ \\
\hline $1679 \mathrm{c}$ & Carbon Monoxide in Nitrogen & $\mathrm{CO}$ & 100 & $\mathrm{ppm}$ \\
\hline $1680 \mathrm{~b}$ & Carbon Monoxide in Nitrogen & $\mathrm{CO}$ & 500 & $\mathrm{ppm}$ \\
\hline $1681 \mathrm{~b}$ & Carbon Monoxide in Nitrogen & $\mathrm{CO}$ & 1000 & $\mathrm{ppm}$ \\
\hline $1683 a$ & Nitric Oxide in Nitrogen & NO & 50 & $\mathrm{ppm}$ \\
\hline$\overline{1684 a}$ & Nitric Oxide in Nitrogen & $\mathrm{NO}$ & 100 & $\mathrm{ppm}$ \\
\hline $1685 \mathrm{~b}$ & Nitric Oxide in Nitrogen & $\mathrm{NO}$ & 250 & $\mathrm{ppm}$ \\
\hline $1686 \mathrm{~b}$ & Nitric Oxide in Nitrogen & $\mathrm{NO}$ & 500 & $\mathrm{ppm}$ \\
\hline $1687 \mathrm{~b}$ & Nitric Oxide in Nitrogen & NO & 1000 & $\mathrm{ppm}$ \\
\hline 1693 & Sulfur Dioxide in Nitrogen & $\mathrm{SO}_{2}$ & 50 & $\mathrm{ppm}$ \\
\hline 1694 & Sulfur Dioxide in Nitrogen & $\mathrm{SO}_{2}$ & 100 & $\mathrm{ppm}$ \\
\hline 1696 & Sulfur Dioxide in Nitrogen & $\mathrm{SO}_{2}$ & 3500 & $\mathrm{ppm}$ \\
\hline$\overline{1805}$ & Benzene in Nitrogen & $\mathrm{C}_{6} \mathrm{H}_{6}$ & 0.25 & $\mathrm{ppm}$ \\
\hline 1806 & Benzene in Nitrogen & $\mathrm{C}_{6} \mathrm{H}_{6}$ & 10 & $\mathrm{ppm}$ \\
\hline 1808 & $\begin{array}{l}\text { Tetrachloroethylene in } \\
\text { Nitrogen-IN PREP }\end{array}$ & $\mathrm{C}_{2} \mathrm{Cl}_{4}$ & 0.25 & $\mathrm{ppm}$ \\
\hline
\end{tabular}




\section{Analyzed Gases (Continued)}

\begin{tabular}{|c|c|c|c|c|}
\hline \multirow{2}{*}{$\frac{\text { SRM }}{2612 a}$} & \multirow{2}{*}{$\frac{\text { Type }}{\text { Carbon Monoxide in Air }}$} & \multirow{2}{*}{$\begin{array}{c}\begin{array}{c}\text { Certified } \\
\text { Component }\end{array} \\
\mathrm{CO} \\
\end{array}$} & \multicolumn{2}{|c|}{ Nominal Concentration } \\
\hline & & & 10 & $\mu$ mole $/$ mole $(\mathrm{ppm})$ \\
\hline$\overline{2613 a}$ & Carbon Monoxide in Air & $\mathrm{CO}$ & 20 & $\mu$ mole $/$ mole $(\mathrm{ppm})$ \\
\hline $2614 a$ & Carbon Monoxide in Air & $\mathrm{CO}$ & 45 & $\mu$ mole $/$ mole $(\mathrm{ppm})$ \\
\hline $2619 \mathrm{a}$ & Carbon Dioxide in Nitrogen & $\mathrm{CO}_{2}$ & 0.5 & mole percent \\
\hline $2620 \mathrm{a}$ & Carbon Dioxide in Nitrogen & $\mathrm{CO}_{2}$ & 1.0 & mole percent \\
\hline $2621 \mathrm{a}$ & Carbon Dioxide in Nitrogen & $\mathrm{CO}_{2}$ & 1.5 & mole percent \\
\hline $2622 \mathrm{a}$ & Carbon Dioxide in Nitrogen & $\mathrm{CO}_{2}$ & 2.0 & mole percent \\
\hline $2623 a$ & Carbon Dioxide in Nitrogen & $\mathrm{CO}_{2}$ & 2.5 & mole percent \\
\hline $2624 \mathrm{a}$ & Carbon Dioxide in Nitrogen & $\mathrm{CO}_{2}$ & 3.0 & mole percent \\
\hline $2625 \mathrm{a}$ & Carbon Dioxide in Nitrogen & $\mathrm{CO}_{2}$ & 3.5 & mole percent \\
\hline $2626 \mathrm{a}$ & Carbon Dioxide in Nitrogen & $\mathrm{CO}_{2}$ & 4.0 & mole percent \\
\hline 2627 & Nitric Oxide in Nitrogen & NO & 5 & $\mu$ mole $/$ mole $(\mathrm{ppm})$ \\
\hline 2628 & Nitric Oxide in Nitrogen & NO & 10 & $\mu \mathrm{mole} / \mathrm{mole}(\mathrm{ppm})$ \\
\hline 2629 & Nitric Oxide in Nitrogen & NO & 20 & $\mu$ mole $/$ mole $(\mathrm{ppm})$ \\
\hline 2630 & Nitric Oxide in Nitrogen & $\mathrm{NO}$ & 1500 & $\mu$ mole $/$ mole $(\mathrm{ppm})$ \\
\hline 2631 & Nitric Oxide in Nitrogen & NO & 3000 & $\mu$ mole $/$ mole $(\mathrm{ppm})$ \\
\hline 2632 & Carbon Dioxide in Nitrogen & $\mathrm{CO}_{2}$ & 300 & ppm \\
\hline 2633 & Carbon Dioxide in Nitrogen & $\mathrm{CO}_{2}$ & 400 & ppm \\
\hline 2634 & Carbon Dioxide in Nitrogen & $\mathrm{CO}_{2}$ & 800 & ppm \\
\hline 2635 & Carbon Monoxide in Nitrogen & $\mathrm{CO}$ & 25 & $\mathrm{ppm}$ \\
\hline 2636 & Carbon Monoxide in Nitrogen & $\mathrm{CO}$ & 250 & $\mathrm{ppm}$ \\
\hline 2637 & Carbon Monoxide in Nitrogen & $\mathrm{CO}$ & 2500 & ppm \\
\hline 2638 & Carbon Monoxide in Nitrogen & $\mathrm{CO}$ & 5000 & $\mathrm{ppm}$ \\
\hline 2639 & Carbon Monoxide in Nitrogen & $\mathrm{CO}$ & 1 & mole percent \\
\hline 2640 & Carbon Monoxide in Nitrogen & $\mathrm{CO}$ & 2 & mole percent \\
\hline 2641 & Carbon Monoxide in Nitrogen & $\mathrm{CO}$ & 4 & mole percent \\
\hline 2642 & Carbon Monoxide in Nitrogen & $\mathrm{CO}$ & 8 & mole percent \\
\hline 2643 & Propane in Nitrogen & $\mathrm{C}_{3} \mathrm{H}_{8}$ & 100 & $\mathrm{ppm}$ \\
\hline 2644 & Propane in Nitrogen & $\mathrm{C}_{3} \mathrm{H}_{8}$ & 250 & $\mathrm{ppm}$ \\
\hline 2645 & Propane in Nitrogen & $\mathrm{C}_{3} \mathrm{H}_{8}$ & 500 & $\mathrm{ppm}$ \\
\hline 2646 & Propane in Nitrogen & $\mathrm{C}_{3} \mathrm{H}_{8}$ & 1000 & $\mathrm{ppm}$ \\
\hline 2647 & Propane in Nitrogen & $\mathrm{C}_{3} \mathrm{H}_{8}$ & 2500 & ppm \\
\hline 2648 & Propane in Nitrogen & $\mathrm{C}_{3} \mathrm{H}_{8}$ & 5000 & $\mathrm{ppm}$ \\
\hline 2649 & Propane in Nitrogen & $\mathrm{C}_{3} \mathrm{H}_{8}$ & 1 & mole percent \\
\hline 2650 & Propane in Nitrogen & $\mathrm{C}_{3} \mathrm{H}_{8}$ & 2 & mole percent \\
\hline 2651 & Propane in Nitrogen and Oxygen & $\mathrm{C}_{3} \mathrm{H}_{8} / \mathrm{O}_{2}$ & $0.01 / 5.0$ & mole percent \\
\hline 2652 & Propane in Nitrogen and Oxygen & $\mathrm{C}_{3} \mathrm{H}_{8} / \mathrm{O}_{2}$ & $0.01 / 10.0$ & mole percent \\
\hline 2653 & Nitrogen Dioxide in Air & $\mathrm{NO}_{2}$ & 250 & ppm \\
\hline 2654 & Nitrogen Dioxide in Air & $\mathrm{NO}_{2}$ & 500 & $\mathrm{ppm}$ \\
\hline 2655 & Nitrogen Dioxide in Air & $\mathrm{NO}_{2}$ & 1000 & $\mathrm{ppm}$ \\
\hline 2656 & Nitrogen Dioxide in Air & $\mathrm{NO}_{2}$ & 2500 & $\mathrm{ppm}$ \\
\hline 2657 & Oxygen in Nitrogen & $\mathrm{O}_{2}$ & 2 & mole percent \\
\hline 2658 & Oxygen in Nitrogen & $\mathrm{O}_{2}$ & 10 & mole percent \\
\hline$\overline{2659}$ & Oxygen in Nitrogen & $\overline{\mathrm{O}_{2}}$ & 21 & mole percent \\
\hline
\end{tabular}




\section{Permeation Tubes}

These SRM's are intended for use in calibrating air pollution monitoring apparatus, and may be used to verify air pollution analytical methods and procedures. Each tube is individually certified.

\section{Sulfur Dioxide}

Sulfur dioxide permeation tubes are available in three lengths $-2,5$, and 10 centimeters. The permeation rates are certified over the temperature range of 20 to $30^{\circ} \mathrm{C}$. The data in the following table are provided as a guide in the selection of the desired SRM. The concentrations generated by typical tubes $(0.28$ micrograms per centimeter per minute) at $25^{\circ} \mathrm{C}$ and at flow rates of 1.5 and 10 liters per minute are shown below.

\begin{tabular}{|c|c|c|c|c|c|c|}
\hline \multirow[t]{2}{*}{ SRM } & \multirow[t]{2}{*}{ Type } & \multirow[t]{2}{*}{$\begin{array}{l}\text { Tube Length } \\
\text { (cm) }\end{array}$} & \multirow[t]{2}{*}{$\begin{array}{c}\text { Permeation } \\
\text { Rate }(\mu \mathrm{g} / \mathrm{min})\end{array}$} & \multicolumn{3}{|c|}{$\begin{array}{l}\text { Typical Concentrations (ppm) } \\
\text { Flow Rates (liters per minute) }\end{array}$} \\
\hline & & & & (1) & (5) & (10) \\
\hline 1625 & Sulfur Dioxide Permeation Tube & 10 & 2.8 & 1.07 & 0.214 & 0.107 \\
\hline 1626 & Sulfur Dioxide Permeation Tube & 5 & 1.4 & 0.535 & 0.107 & 0.0535 \\
\hline 1627 & Sulfur Dioxide Permeation Tube & 2 & 0.56 & 0.214 & 0.0428 & 0.0214 \\
\hline
\end{tabular}

\section{Nitrogen Dioxide}

Nitrogen dioxide permeation device (SRM 1629a) is calibrated at $25.0^{\circ} \mathrm{C}$ only. The temperature coefficient given with each tube provides the means to calculate permeation rates at other temperatures near $25^{\circ} \mathrm{C}$. The permeation rates for these tubes are between 0.5 and $1.5 \mathrm{mg} / \mathrm{min}$ at $25^{\circ} \mathrm{C}$. A tube with a rate of $1.0 \mu \mathrm{g} / \mathrm{min}$, in an air-flow of one liter per minute at $25^{\circ} \mathrm{C}$, will produce a concentration of $0.5 \mathrm{ppm}$ of $\mathrm{NO}_{2}$. $(\mathrm{Cannot}$ be shipped by air.)

\section{Benzene}

SRM 1911 is a permeation device that is certified for its permeation rate in micrograms of benzene at $25^{\circ} \mathrm{C}$. It is intended for use in the preparation of gases of known benzene content and for the standardization of air pollution an related chemical analyses. A typical permeation rate for this SRM is $35 \mu \mathrm{g} /$ minute at $25^{\circ} \mathrm{C}$. (Cannot be shipped by air.)

\section{Analyzed Liquids and Solids}

These SRM's are intended for use in the analysis of materials for constituents of interest in health or environmental problems. See also: Clinical SRM's page 56, and Industrial Hygiene SRM's page 66

\section{Single Element}

\begin{tabular}{|c|c|c|c|c|c|}
\hline \multirow[b]{2}{*}{ SRM } & \multirow[b]{2}{*}{ Type } & \multirow[b]{2}{*}{ Unit Size } & \multicolumn{3}{|c|}{ Certified Element } \\
\hline & & & Lead & Sulfur & Mercury \\
\hline 1579 & Powdered Lead Base Paint & $35 \mathrm{~g}$ & $11.87 \%$ & & \\
\hline$\overline{1618}$ & $\begin{array}{l}\text { Vanadium and Nickel in } \\
\text { Residual Fuel Oil }\end{array}$ & IN PREP & & & \\
\hline 1619 & Sulfur in Residual Fuel Oil & $100 \mathrm{~mL}$ & & $0.719 \%$ & \\
\hline $1620 a$ & Sulfur in Residual Fuel Oil & $100 \mathrm{~mL}$ & & $4.504 \%$ & \\
\hline $1621 b$ & Sulfur in Residual Fuel Oil & $100 \mathrm{~mL}$ & & $0.950 \%$ & \\
\hline $1622 b$ & Sulfur in Residual Fuel Oil & $100 \mathrm{~mL}$ & & $1.982 \%$ & \\
\hline $1623 a$ & Sulfur in Residual Fuel Oil & $100 \mathrm{~mL}$ & & $0.240 \%$ & \\
\hline $1624 a$ & Sulfur in Distillate Fuel Oil & $100 \mathrm{~mL}$ & & $0.141 \%$ & \\
\hline$\overline{1630}$ & Trace Mercury in Coal & $50 \mathrm{~g}$ & & & $0.13 \mu \mathrm{g} / \mathrm{g}$ \\
\hline
\end{tabular}




\section{Single Element (Continued)}

\begin{tabular}{|c|c|c|c|c|c|}
\hline \multirow[b]{2}{*}{ SRM } & \multirow[b]{2}{*}{ Type } & \multirow[b]{2}{*}{ Unit Size } & \multicolumn{3}{|c|}{ Certified Element } \\
\hline & & & Lead & Sulfur & Mercury \\
\hline $1636 a$ & Lead in Reference Fuel & 3 vials each & $\begin{array}{l}0.03,0.05 \\
0.07,2.0 \\
\mathrm{~g} / \mathrm{gal}\end{array}$ & & \\
\hline $1637 a$ & Lead in Reference Fuel & 4 vials each & $\begin{array}{l}0.03,0.05 \\
0.07 \mathrm{~g} / \mathrm{gal}\end{array}$ & & \\
\hline $1638 \mathrm{a}$ & Lead in Reference Fuel & 12 vials each & $2.0 \mathrm{~g} / \mathrm{gal}$ & & \\
\hline $1641 b$ & Mercury in Water $(\mu \mathrm{g} / \mathrm{mL})$ & $6 \times 20 \mathrm{~mL}$ & & & $1.52 \mu \mathrm{g} / \mathrm{mL}$ \\
\hline $1642 b$ & Mercury in Water $(\mathrm{ng} / \mathrm{mL})$ & $950 \mathrm{~mL}$ & & & $1.49 \mathrm{ng} / \mathrm{mL}$ \\
\hline 8505 & Vanadium in Crude Oil & IN PREP & & & \\
\hline
\end{tabular}

Concentrations in weight percent, or as otherwise noted.

\section{Multi-Element}

\begin{tabular}{|c|c|c|c|c|c|c|c|c|}
\hline SRM & $1632 \mathrm{a}$ & $1633 a$ & $1634 a$ & 1635 & $1643 a$ & 1645 & 1646 & 1648 \\
\hline Type & $\begin{array}{l}\text { Trace } \\
\text { Elements } \\
\text { in Coal } \\
\text { (Bituminous) }\end{array}$ & $\begin{array}{l}\text { Trace } \\
\text { Elements } \\
\text { in Coal Fly } \\
\text { Ash }\end{array}$ & $\begin{array}{l}\text { Trace } \\
\text { Elements } \\
\text { in Fuel Oil }\end{array}$ & $\begin{array}{l}\text { Trace } \\
\text { Elements } \\
\text { in Coal } \\
\text { (Sub- } \\
\text { bituminous) }\end{array}$ & $\begin{array}{l}\text { Trace } \\
\text { Elements } \\
\text { in Water } \\
(\mathrm{ng} / \mathrm{g})\end{array}$ & $\begin{array}{l}\text { River } \\
\text { Sediment }\end{array}$ & $\begin{array}{l}\text { Estuarine } \\
\text { Sediment }\end{array}$ & $\begin{array}{l}\text { Urban } \\
\text { Particulate }\end{array}$ \\
\hline Unit Size & $75 \mathrm{~g}$ & $75 \mathrm{~g}$ & $100 \mathrm{~mL}$ & $75 \mathrm{~g}$ & $950 \mathrm{~mL}$ & $70 \mathrm{~g}$ & $75 \mathrm{~g}$ & $2 \mathrm{~g}$ \\
\hline ELEMENT & & & & & & & & \\
\hline Aluminum & $(3.1 \%)$ & $(14 \%)$ & & $(0.32 \%)$ & & $2.26 \%$ & $6.25 \%$ & $3.42 \%$ \\
\hline Antimony & $(0.6)$ & (7) & & $(0.14)$ & & $(51)$ & $(0.4)$ & $(45)$ \\
\hline Arsenic & 9.3 & 145 & $(0.12)$ & 0.42 & $76 \mathrm{ng} / \mathrm{g}$ & (66) & 11.6 & 115 \\
\hline Barium & & $(0.15 \%)$ & & & $46 \mathrm{ng} / \mathrm{g}$ & & & $(737)$ \\
\hline Beryllium & 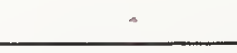 & $(12)$ & $(0.006)$ & & $19 \mathrm{ng} / \mathrm{g}$ & & $(1.5)$ & \\
\hline Bromine & & & $(<1)$ & & & & & $(500)$ \\
\hline Cadmium & 0.17 & 1.0 & $(0.002)$ & 0.03 & $10 \mathrm{ng} / \mathrm{g}$ & 10.2 & 0.36 & 75 \\
\hline Calcium & $0.23 \%$ & $1.11 \%$ & $(16)$ & & & $(2.9)$ & $0.83 \%$ & \\
\hline Cerium & $(30)$ & $(180)$ & & (3.6) & & & $(80)$ & $(55)$ \\
\hline Cesium & $(2.4)$ & $(11)$ & & & & & $(3.7)$ & (3) \\
\hline Chlorine & & & $(31)$ & & & & & $(0.45 \%)$ \\
\hline Chromium & 34.4 & 196 & $(0.7)$ & 2.5 & $17 \mathrm{ng} / \mathrm{g}$ & $2.96 \%$ & 76 & 403 \\
\hline Cobalt & $(6.8)$ & $(46)$ & $(0.3)$ & $(0.65)$ & $19 \mathrm{ng} / \mathrm{g}$ & 10.1 & 10.5 & $(18)$ \\
\hline Copper & 16.5 & 118 & & 3.6 & $18 \mathrm{ng} / \mathrm{g}$ & 109 & 18 & 609 \\
\hline Europium & $(0.5)$ & (4) & & $(0.06)$ & & & $(1.5)$ & $(0.8)$ \\
\hline Fluorine & & & & & & $(0.09 \%)$ & & \\
\hline Gallium & $(8.5)$ & $(58)$ & & $(1.05)$ & & & & \\
\hline Germanium & & & & & & & $(1.4)$ & \\
\hline Hafnium & $(1.6)$ & $(7.6)$ & & $(0.29)$ & & & & $(4.4)$ \\
\hline Indium & & & & & & & & $(1.0)$ \\
\hline
\end{tabular}




\section{Multi-Element (Continued)}

\begin{tabular}{|c|c|c|c|c|c|c|c|c|}
\hline SRM & $1632 \mathrm{a}$ & $1633 a$ & $1634 a$ & 1635 & $1643 a$ & 1645 & 1646 & 1648 \\
\hline Туре & $\begin{array}{l}\text { Trace } \\
\text { Elements } \\
\text { in Coal } \\
\text { (Bituminous) }\end{array}$ & $\begin{array}{l}\text { Trace } \\
\text { Elements } \\
\text { in Coal Fly } \\
\text { Ash }\end{array}$ & $\begin{array}{l}\text { Trace } \\
\text { Elements } \\
\text { in Fuel Oil }\end{array}$ & $\begin{array}{l}\text { Trace } \\
\text { Elements } \\
\text { in Coal } \\
\text { (Sub- } \\
\text { bituminous) }\end{array}$ & $\begin{array}{l}\text { Trace } \\
\text { Elements } \\
\text { in Water } \\
(\mathrm{ng} / \mathrm{g})\end{array}$ & $\begin{array}{l}\text { River } \\
\text { Sediment }\end{array}$ & $\begin{array}{l}\text { Estuarine } \\
\text { Sediment }\end{array}$ & $\begin{array}{l}\text { Urban } \\
\text { Particulate }\end{array}$ \\
\hline Unit Size & $75 \mathrm{~g}$ & $75 \mathrm{~g}$ & $100 \mathrm{~mL}$ & $75 \mathrm{~g}$ & $950 \mathrm{~mL}$ & $70 \mathrm{~g}$ & $75 \mathrm{~g}$ & $2 \mathrm{~g}$ \\
\hline Iodine & & & & & & & & $(20)$ \\
\hline Iron & $1.11 \%$ & $9.4 \%$ & $(31)$ & $0.239 \%$ & $88 \mathrm{ng} / \mathrm{g}$ & $11.3 \%$ & $3.35 \%$ & $3.91 \%$ \\
\hline Lanthanum & & & & & & (9) & & $(42)$ \\
\hline Lead & 12.4 & 72.4 & 2.8 & 1.9 & $27 \mathrm{ng} / \mathrm{g}$ & 714 & 28.2 & $0.655 \%$ \\
\hline Lithium & & & & & & & $(49)$ & \\
\hline Magnesium & $(0.1 \%)$ & $0.455 \%$ & & & & $0.74 \%$ & $1.09 \%$ & $(0.8 \%)$ \\
\hline Manganese & 28 & $(190)$ & 0.19 & 21.4 & $31 \mathrm{ng} / \mathrm{g}$ & 785 & 375 & $(860)$ \\
\hline Mercury & 0.13 & 0.16 & $(<0.002)$ & & $(<0.2) \mathrm{ng} / \mathrm{g}$ & 1.1 & 0.063 & \\
\hline Molybdenum & & $(29)$ & $(0.12)$ & & $95 \mathrm{ng} / \mathrm{g}$ & & $(2)$ & \\
\hline Nickel & 19.4 & 127 & 29 & 1.74 & $55 \mathrm{ng} / \mathrm{g}$ & 45.8 & 32 & 82 \\
\hline Phosphorus & & & & & & & $0.054 \%$ & \\
\hline Potassium & $0.42 \%$ & $1.88 \%$ & & & & $1.26 \%$ & $(1.4 \%)$ & $1.05 \%$ \\
\hline Rubidium & $(31)$ & 131 & & & & & $(87)$ & $(52)$ \\
\hline Samarium & & & & & & & & $(4.4)$ \\
\hline Scandium & $(6.3)$ & $(40)$ & & $(0.63)$ & & $(2)$ & $(10.8)$ & $(7)$ \\
\hline Selenium & 2.6 & 10.3 & 0.15 & 0.9 & $11 \mathrm{ng} / \mathrm{g}$ & $(1.5)$ & $(0.6)$ & 27 \\
\hline Silicon & & $22.8 \%$ & & & & & $(31 \%)$ & \\
\hline Silver & & & & & $2.8 \mathrm{ng} / \mathrm{g}$ & & & (6) \\
\hline Sodium & 840 & $0.17 \%$ & 87 & $(0.24 \%)$ & & $0.54 \%$ & $(2 \%)$ & $0.425 \%$ \\
\hline Strontium & & 830 & & & $239 \mathrm{ng} / \mathrm{g}$ & & & \\
\hline Sulfur & $1.58 \%$ & & $2.85 \%$ & $0.33 \%$ & & (1.1) & $(0.96 \%)$ & $(5 \%)$ \\
\hline Tellurium & & & & & & & $(0.5)$ & \\
\hline Thallium & & 5.7 & & & & 1.44 & $(0.5)$ & \\
\hline Thorium & 4.5 & 24.7 & & 0.62 & & 1.62 & $(10)$ & $(7.4)$ \\
\hline Titanium & $(0.18 \%)$ & $(0.8 \%)$ & & $(0.02 \%)$ & & & $(0.51 \%)$ & $(0.40 \%)$ \\
\hline Tungsten & & & & & & & & $(4.8)$ \\
\hline Uranium & 1.28 & 10.2 & & 0.24 & & 1.11 & & 5.5 \\
\hline Vanadium & 44 & $(300)$ & 56 & 5.2 & $53 \mathrm{ng} / \mathrm{g}$ & 23.5 & 94 & 140 \\
\hline Zinc & 28 & 220 & 2.7 & 4.7 & $68 \mathrm{ng} / \mathrm{g}$ & $0.172 \%$ & 138 & $0.476 \%$ \\
\hline
\end{tabular}

Values in parentheses are not certified, but are given for information only.

Concentrations in microgram per gram, or where noted weight percent.

All concentrations for SRM 1643a are nanogram per gram. 


\section{Organic Constituents}

\begin{tabular}{|lll|}
\hline SRM & \multicolumn{1}{c|}{ Type } & \multicolumn{1}{c|}{ Unit of Issue } \\
\hline 1580 & Shale Oil & Set of $5,2 \mathrm{~mL} /$ ampoule \\
\hline 1582 & Petroleum Crude Oil & IN PREP \\
\hline 1644 & Polynuclear Aromatic Hydrocarbon Generator Columns & Set of 3 columns \\
\hline 1647 & Priority Pollutant Polynuclear Aromatic Hydrocarbons (in Acetonitrile) & Set of $5,1.2 \mathrm{~mL} /$ ampoule \\
\hline 1649 & Urban Dust/Organics & 10 grams \\
\hline 1639 & Halocarbons (in methanol) for Water Analysis & Set of $5,1.5 \mathrm{~mL} / \mathrm{ampoule}$ \\
\hline
\end{tabular}

\begin{tabular}{|c|c|c|c|c|}
\hline \multicolumn{5}{|c|}{ SRM's Certified for Organic Constituents } \\
\hline SRM & 1580 & 1644 & 1647 & 1649 \\
\hline Constituents & $(\mu \mathrm{g} / \mathrm{g})$ & $(\mu \mathrm{g} / \mathrm{kg})$ & $(\mu \mathrm{g} / \mathrm{mL})$ & $(\mu \mathrm{g} / \mathrm{g})$ \\
\hline Anthracene & & 16.6 to 60.1 & 3.29 & \\
\hline Benz [a] anthracene & & 3.38 to 12.8 & 5.03 & 2.6 \\
\hline Benzo[a]pyrene & 21 & 0.59 to 2.26 & 5.3 & 2.9 \\
\hline Benzo[e]pyrene & 18 & & & \\
\hline Fluoranthene & 54 & & 10.1 & 7.1 \\
\hline o.Cresol & 385 & & & \\
\hline Phenol & 407 & & & \\
\hline Perylene & 3.4 & & & \\
\hline Pyrene & 104 & & 9.84 & \\
\hline 2,6-Dimethylphenol & 175 & & & \\
\hline Benzo[f]quinoline & 16 & & & \\
\hline \multicolumn{5}{|l|}{ (5,6-Benzoquinoline) } \\
\hline Naphthalene & & & 22.5 & \\
\hline Acenaphthylene & & & 19.1 & \\
\hline Acenaphthene & & & 21.0 & \\
\hline Fluorene & & & 4.92 & \\
\hline Phenanthrene & & & 5.06 & \\
\hline Chrysene & & & 4.68 & \\
\hline Benzo[b]fluoranthene & & & 5.11 & \\
\hline Benzo[k] fluoranthene & & & 5.02 & \\
\hline Benzo[ghi]perylene & & & 4.01 & 4.5 \\
\hline Dibenz $[a, h]$ anthracene & & & 3.68 & \\
\hline Indeno[ 1,2,3-cd]pyrene & & & 4.06 & 3.3 \\
\hline
\end{tabular}

${ }^{1}$ Range of aqueous concentrations certified also in $\mathrm{nmol} / \mathrm{L}$. 


\section{Organic Constituents (Continued)}

\begin{tabular}{lc}
\hline SRM $\mathbf{1 6 3 9 - C e r t i f i e d ~ C o n c e n t r a t i o n ~ o f ~ H a l o c a r b o n s ~ a t ~} \mathbf{2 3} \pm \mathbf{3}{ }^{\circ} \mathbf{C}$ & \\
\hline Compound & Concentration, $\mathbf{n g} / \boldsymbol{\mu L}$ \\
\hline Chloroform & 6235 \\
\hline Chlorodibromomethane & 124.6 \\
\hline Bromodichloromethane & 389.9 \\
\hline Bromoform & 86.5 \\
\hline Carbon tetrachloride & 157.0 \\
\hline Trichloroethylene & 85.8 \\
\hline Tetrachloroethylene & 40.6 \\
\hline
\end{tabular}

\section{Polychlorinated Biphenyls in Oil}

SRM 1581 was developed primarily for use in calibrating instrumentation and validating methodology for the determination of polychlorinated biphenyls (PCB's) in oil. It lists the certified concentrations of Aroclor 1242 and Aroclor 1260 present individually at $100 \mu \mathrm{g} / \mathrm{g}(\mathrm{ppm})$ in motor oil and transformer oil. Additional base oil is supplied to dilute the four certified concentrates.

\section{Sulfur in Coal}

This series (SRM's 2682-2685) is intended primarily for use as analytical standards for determination of total sulfur in coal, ash content, and calorific value (BTU/lb). SRM's 2682-2685 are four different coals that were crushed and ground to pass a 60-mesh sieve. Each coal SRM has been analyzed for approximately 30 elements, including carbon, hydrogen and nitrogen. Semi-quantitative data are provided for information.

\begin{tabular}{|c|c|c|c|c|c|}
\hline \multirow[b]{2}{*}{ SRM } & \multirow[b]{2}{*}{ Coal Type } & \multirow{2}{*}{$\begin{array}{l}\text { Sulfur } \\
\text { Wt.\% }\end{array}$} & \multirow{2}{*}{$\begin{array}{l}\text { Furnace } \\
\text { Ash Wt.\% }\end{array}$} & \multicolumn{2}{|c|}{ HHV2 } \\
\hline & & & & $\mathbf{M J} \cdot \mathbf{K g}^{-1}$ & $\left(\mathrm{BTU} \bullet l \mathrm{~b}^{-1}\right)$ \\
\hline 2682 & Sub-bituminous & 0.47 & 6.37 & 27.45 & $(11800)$ \\
\hline 2683 & Bituminous & 1.85 & 6.85 & 32.70 & $(14060)$ \\
\hline 2684 & Bituminous & 3.00 & 11.09 & 29.68 & $(12760)$ \\
\hline 2685 & Bituminous & 4.62 & 16.53 & 28.15 & $(12100)$ \\
\hline
\end{tabular}

NOTE: The calorific values (MJ. $\mathrm{Kg}^{-1}$ ) may decrease upon the aging or normal oxidation of the coals. NBS will continue to monitor these calorific values and report any substantive change to the purchaser.

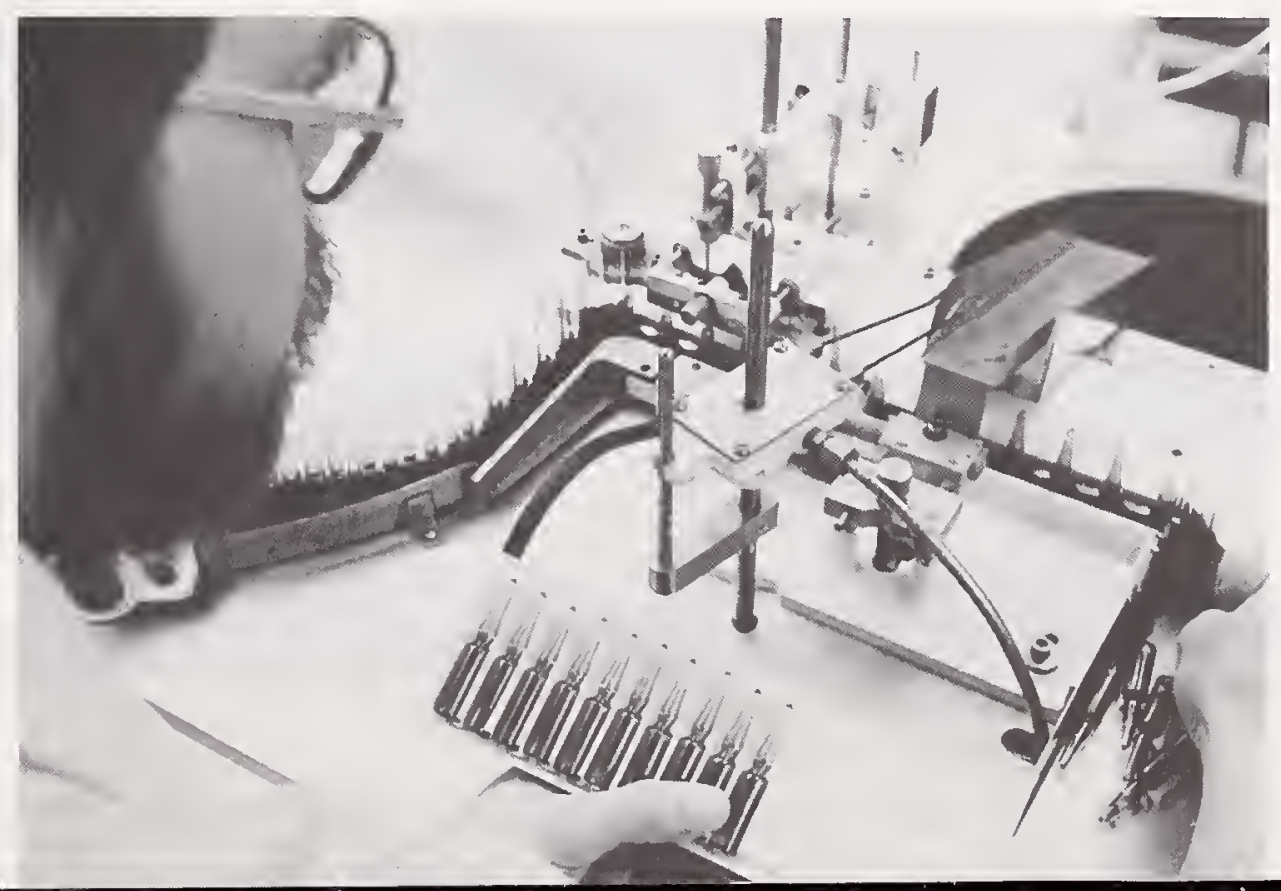

Dale Friend operates equipment used to sea ampules containing various clinical, biological, and environmental SRM's. 
Industriall Hygiene

These SRM's were developed especially for clinical and industrial hygiene analyses, and are the outgrowth of an NBS program to develop standardization and reference materials for toxicology research and for monitoring human exposure to selected toxic elements in the workplace environment.

\section{Freeze-Dried Urine}

SRM's 2670, 2671a, and 2672a consists of ireeze-dried urine in $30 \mathrm{~mL}$ serum bottles. The freeze-dried urine SRM's are to be reconstituted by the addition of $20 \mathrm{~mL}$ of pure water to each bottle. Each unit contains a set of four bottles, two bottles each at normal and elevated levels.

\begin{tabular}{|lll|}
\hline SRM & Matrix & \multicolumn{1}{c|}{ Constituent Certified or Determined } \\
\hline 2670 & Freeze-Dried Urine & $\begin{array}{l}\text { Arsenic, Cadmium, Calcium, Chloride, Copper, Lead, } \\
\text { Magnesium, Manganese, Nickel, Potassium, Selenium, } \\
\text { Sodium, Zinc }\end{array}$ \\
\hline 2671 a & Freeze-Dried Urine & Fluoride \\
\hline $2672 \mathrm{a}$ & Freeze-Dried Urine & Mercury \\
\hline
\end{tabular}

\section{Materials on Filter Media}

These SRM's consist of potentially hazardous materials deposited on filters to be used to determine the levels of these materials in industrial atmosphere.

\begin{tabular}{|c|c|c|c|c|c|c|c|}
\hline \multirow[b]{2}{*}{ SRM } & \multirow[b]{2}{*}{ Type } & \multirow{2}{*}{$\begin{array}{l}\text { Unit } \\
\text { Size }\end{array}$} & \multirow{2}{*}{$\begin{array}{l}\text { Material } \\
\text { Certified }\end{array}$} & \multicolumn{4}{|c|}{ Quantity Certified ( $\mu \mathrm{g} /$ filter) } \\
\hline & & & & I & III & III & IV \\
\hline \multirow[t]{2}{*}{2673} & Sulfate and Nitrate & 2 filters at & Sulfate & 503 & 2002 & 6939 & 2 \\
\hline & on Filter Media & each level & Nitrate & 100 & 1002 & 2513 & 2 \\
\hline \multirow[t]{2}{*}{2674} & Lead on Filter Media & 2 filters at & & & & & \\
\hline & & each level & Lead & 100 & 303 & 1505 & 1.4 \\
\hline \multirow[t]{2}{*}{2675} & Beryllium on Filter & Set of 3 & Beryllium & 0.052 & 0.25 & 1.0 & \\
\hline & Media & & & & & & \\
\hline \multirow[t]{4}{*}{$2676 \mathrm{~b}$} & Metals on Filter Media & Set of 12 & Cadmium & 0.99 & 2.49 & 10.14 & $(<0.01)$ \\
\hline & & & Lead & 7.55 & 14.9 & 30.4 & $(<0.04)$ \\
\hline & & & Manganese & 1.88 & 9.41 & 18.5 & $(<0.01)$ \\
\hline & & & Zinc & 10.01 & 49.7 & 99.5 & $(0.4)$ \\
\hline $2679 a$ & Quartz on Filter Media & Set of 4 & IN PREP & & & & \\
\hline
\end{tabular}

Values in parentheses are not certified, but are given for information only.

\section{Respirable Quartz}

This SRM consists of quartz powder that is in the respirable size range. It is intended for use in determining the level of quartz in an industrial atmosphere by $x$-ray diffraction.

\begin{tabular}{|llr|}
\hline SRM & Type & Amount \\
\hline 1878 & Alpha Quartz & $5 \mathrm{~g}$ \\
\hline
\end{tabular}




\section{Asbestos}

This SRM consists of four $3 \times 3 \mathrm{~mm}$ sections of a $0.4 \mathrm{~mm}$ pore size polycarbonate filter containing chrysotile fibers mixed with an urban dust. It is intended for use in evaluating the techniques used to count and identify chrysotile asbestos fibers in filter samples by transmission electron microscopy.

\begin{tabular}{|lcr|}
\hline SRM & Type & Fiber Loading \\
\hline 1876 & Chrysotile Asbestos & 30 fibers $/ 0.01 \mathrm{~mm}^{2}$ \\
\hline
\end{tabular}

These SRM's are intended for the preparation of solutions in oils of known and reproducible concentrations of metals. Because "matrix" effects occur, it is desirable to prepare the standard solutions in oil identical or similar to the oil being studied. Possession of an adequate collection of these metallo-organic SRM's permits the preparation of any desired blend of known concentrations of metal in the appropriate lubricating oil. They are used primarily for the calibration of spectrochemical equipment used in the determination of metals in lubricating oil. This technique is used extensively in the defense program, the transportation industry, and other industries where the consequences of failure of a moving metal part may range from inconvenient to catastrophic.

The Certificate supplied with each SRM gives the percentage of the element of interest and directions for preparing a solution of known concentration in lubricating oil.

\begin{tabular}{|c|c|c|c|c|}
\hline \multirow[b]{2}{*}{ SRM } & \multirow[b]{2}{*}{ Type } & \multicolumn{3}{|c|}{ Constituent Certified } \\
\hline & & Element & (Wt. percent) & $\begin{array}{c}\text { Wt/Unit } \\
\text { (grams) }\end{array}$ \\
\hline $1075 \mathrm{a}$ & Aluminum 2-ethylhexanoate & Aluminum & 8.07 & 5 \\
\hline $1051 \mathrm{~b}$ & Barium cyclohexanebutyrate & Barium & 28.7 & 5 \\
\hline $1053 \mathrm{a}$ & Cadmium cyclohexanebutyrate & Cadmium & 24.8 & 5 \\
\hline $1074 \mathrm{a}$ & Calcium 2-ethylhexanoate & Calcium & 12.5 & 5 \\
\hline $1078 \mathrm{~b}$ & Tris(1-phenyl-1,3-butanediono)chromium (III) & Chromium & 9.6 & 5 \\
\hline $1055 \mathrm{~b}$ & Cobalt cyclohexanebutyrate & Cobalt & 14.8 & 5 \\
\hline $1080 \mathrm{a}$ & Bis(1-phenyl-1,3-butanediono) copper (II) & Copper & 16.37 & 5 \\
\hline $1079 \mathrm{~b}$ & Tris (1-phenyl-1,3-butanediono) & Iron & 10.45 & 5 \\
\hline $1059 \mathrm{c}$ & Lead cyclohexanebutyrate & Lead & & 5 \\
\hline $1061 \mathrm{c}$ & Magnesium cyclohexanebutyrate & Magnesium & 6.45 & 5 \\
\hline $1062 \mathrm{~b}$ & Manganous cyclohexanebutyrate & Manganese & 13.2 & 5 \\
\hline $1065 \mathrm{~b}$ & Nickel cyclohexanebutyrate & Nickel & 13.89 & 5 \\
\hline $1071 \mathrm{~b}$ & Triphenyl phosphate & Phosphorus & 9.48 & 5 \\
\hline 1076 & Potassium Erucate & Potassium & IN PREP & \\
\hline $1066 \mathrm{a}$ & Octaphenylcyclotetrasiloxane & Silicon & 14.14 & 5 \\
\hline $1077 \mathrm{a}$ & Silver 2-ethylhexanoate & Silver & 42.60 & 5 \\
\hline $1069 \mathrm{~b}$ & Sodium cyclohexanebutyrate & Sodium & 12.0 & 5 \\
\hline $1070 \mathrm{a}$ & Strontium cyclohexanebutyrate & Strontium & 20.7 & 5 \\
\hline $1057 \mathrm{~b}$ & Dibutyltin bis (2-ethylhexanoate) & Tin & 22.95 & 5 \\
\hline $1052 \mathrm{~b}$ & Bis (1-phenyl-1,3-butanediono) & Vanadium & 13.01 & 5 \\
\hline $1073 b$ & Zinc cyclohexanebutyrate & Zinc & 16.66 & 5 \\
\hline
\end{tabular}




\section{Wear-Metals in Oil}

These SRM's consist of 10 metallo-organic compounds blended into a base oil. Element values are listed in $\mu \mathrm{g} / \mathrm{g}$.

\begin{tabular}{|c|c|c|c|}
\hline SRM & 1083 & 1084 & 1085 \\
\hline Type & $\begin{array}{c}\text { Base Oil } \\
\text { (IN PREP) }\end{array}$ & $\begin{array}{c}\text { Wear-Metals in } \\
\text { Lubricating Oil } \\
100 \text { ppm }\end{array}$ & $\begin{array}{c}\text { Wear-Metals in } \\
\text { Lubricating Oil } \\
300 \mathrm{ppm}\end{array}$ \\
\hline Unit Size & $150 \mathrm{~mL}$ & $85 \mathrm{~mL}$ & $85 \mathrm{~mL}$ \\
\hline \multicolumn{4}{|l|}{ ELEMENT } \\
\hline Aluminum & & 98 & 296 \\
\hline Chromium & & 100 & 298 \\
\hline Copper & & 98 & 295 \\
\hline Iron & & 100 & 300 \\
\hline Lead & & 101 & 305 \\
\hline Magnesium & & 98 & 297 \\
\hline Molybdenum & & 97 & 292 \\
\hline Nickel & & 101 & 303 \\
\hline Silver & & $(102)$ & $(296)$ \\
\hline Titanium & & 99 & \\
\hline
\end{tabular}

Values in parentheses are not certified, but are given for information only.

\section{Fertilizers}

These SRM's are intended for use in the fertilizer industry as working standards for the determination of the certified constituents.

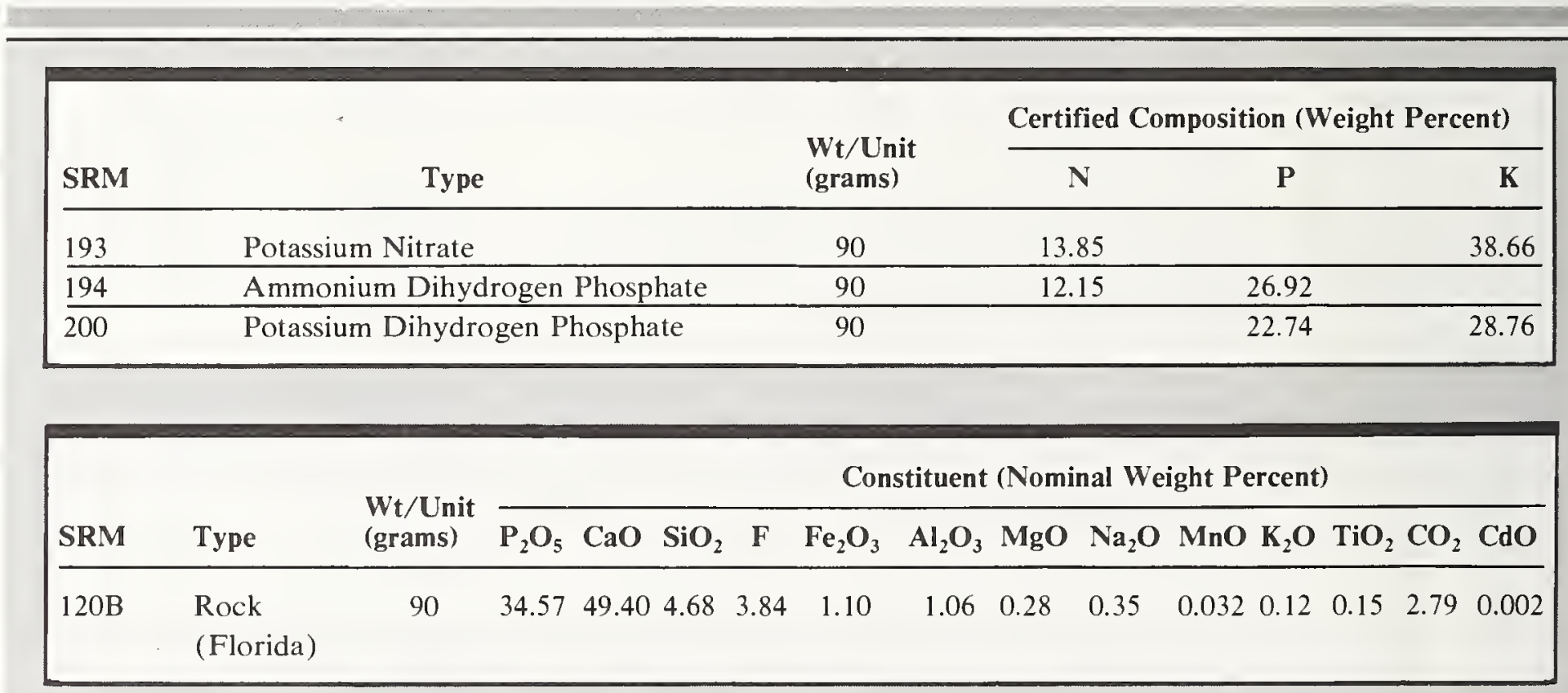


These SRM's are intended for use in evaluating the accuracy of assay methods. They are certified for the elements that are of economic interest, and occasionally, have data given for information only. These SRM's are supplied in the form of fine powders, usually less than $0.15 \mathrm{~mm}$.

\begin{tabular}{|c|c|c|c|c|c|}
\hline SRM & $79 a$ & 180 & 181 & 182 & 183 \\
\hline Type & $\begin{array}{l}\text { Fluorspar, } \\
\text { customs } \\
\text { grade }\end{array}$ & $\begin{array}{l}\text { Fluorspar, } \\
\text { high grade }\end{array}$ & $\begin{array}{l}\text { Lithium } \\
\text { ore } \\
\text { (Spodumene) }\end{array}$ & $\begin{array}{l}\text { Lithium } \\
\text { ore } \\
\text { (Petalite) }\end{array}$ & $\begin{array}{l}\text { Lithium } \\
\text { ore } \\
\text { (Lepidolite) }\end{array}$ \\
\hline Unit Weight & $120 \mathrm{~g}$ & $120 \mathrm{~g}$ & $45 \mathrm{~g}$ & $45 \mathrm{~g}$ & $45 \mathrm{~g}$ \\
\hline \multicolumn{6}{|l|}{ Constituents } \\
\hline$\overline{\mathrm{CaF}_{2}}$ & 97.39 & 98.80 & & & \\
\hline $\mathrm{Li}_{2} \mathrm{O}$ & & & 6.39 & 4.34 & 4.12 \\
\hline \multicolumn{6}{|l|}{$\mathrm{Cu}$} \\
\hline \multicolumn{6}{|l|}{$\overline{\mathrm{Re}}$} \\
\hline \multicolumn{6}{|l|}{ Mo } \\
\hline \multicolumn{6}{|l|}{$\overline{\mathrm{Au}}$} \\
\hline$\overline{\mathrm{Ag}}$ & & & & & \\
\hline
\end{tabular}

\begin{tabular}{|c|c|c|c|c|}
\hline SRM & 330 & 331 & 332 & 333 \\
\hline Type & $\begin{array}{l}\text { Copper, } \\
\text { ore mill } \\
\text { heads }\end{array}$ & $\begin{array}{l}\text { Copper, } \\
\text { ore mill } \\
\text { tails }\end{array}$ & $\begin{array}{l}\text { Copper, } \\
\text { Concentrate }\end{array}$ & $\begin{array}{l}\text { Molybdenum, } \\
\text { Concentrate }\end{array}$ \\
\hline Unit Weight & $100 \mathrm{~g}$ & $100 \mathrm{~g}$ & $50 \mathrm{~g}$ & $35 \mathrm{~g}$ \\
\hline \multicolumn{5}{|l|}{ Constituents } \\
\hline \multicolumn{5}{|l|}{$\overline{\mathrm{CaF}_{2}}$} \\
\hline \multicolumn{5}{|l|}{$\mathrm{Li}_{2} \mathrm{O}$} \\
\hline $\mathrm{Cu}$ & 0.84 & 0.091 & 28.4 & 1.038 \\
\hline$\overline{\operatorname{Re}}$ & $0.30 \mathrm{ppm}$ & $0.04 \mathrm{ppm}$ & $10.2 \mathrm{ppm}$ & 0.087 \\
\hline$\overline{\mathrm{Mo}}$ & 0.018 & 0.0022 & 0.64 & 55.3 \\
\hline $\mathrm{Au}$ & $(0.093 \mathrm{ppm})$ & $(0.034 \mathrm{ppm})$ & $(2.14 \mathrm{ppm})$ & $(8.9 \mathrm{ppm})$ \\
\hline$\overline{\mathrm{Ag}}$ & $(1.37 \mathrm{ppm})$ & $(0.243 \mathrm{ppm})$ & $(38.7 \mathrm{ppm})$ & $(25.0 \mathrm{ppm})$ \\
\hline
\end{tabular}

Values in parentheses are not certified, but are given for information only. 


\section{Ores}

\begin{tabular}{|c|c|c|c|c|c|c|c|}
\hline SRM & $27 f$ & 690 & 691 & 692 & 693 & $69 b$ & 696 \\
\hline Type & $\begin{array}{l}\text { Iron Ore, } \\
\text { Sibley }\end{array}$ & $\begin{array}{l}\text { Iron Ore, } \\
\text { Canada }\end{array}$ & $\begin{array}{l}\text { Iron Oxide } \\
\text { reduced }\end{array}$ & $\begin{array}{l}\text { Iron Ore, } \\
\text { Labrador }\end{array}$ & $\begin{array}{l}\text { Iron Ore, } \\
\text { Nimba }\end{array}$ & $\begin{array}{l}\text { Bauxite, } \\
\text { Arkansas }\end{array}$ & $\begin{array}{l}\text { Bauxite, } \\
\text { Surinam }\end{array}$ \\
\hline Unit Weight & $100 \mathrm{~g}$ & $150 \mathrm{~g}$ & $100 \mathrm{~g}$ & $150 \mathrm{~g}$ & $150 \mathrm{~g}$ & $60 \mathrm{~g}$ & $60 \mathrm{~g}$ \\
\hline \multicolumn{8}{|l|}{ Constituents } \\
\hline $\mathrm{Al}_{2} \mathrm{O}_{3}$ & 0.82 & 0.18 & 1.22 & 1.41 & 1.02 & 48.8 & 54.5 \\
\hline $\mathrm{BaO}$ & & & & & & $(0.008)$ & $(0.004)$ \\
\hline \multicolumn{8}{|l|}{$\mathrm{Cd}$} \\
\hline \multicolumn{8}{|l|}{$\mathrm{CdO}$} \\
\hline $\mathrm{CaO}$ & 0.039 & 0.20 & 0.63 & 0.023 & 0.016 & 0.13 & 0.018 \\
\hline$\overline{\mathrm{Co}}$ & - & & 0.030 & & & $(0.0001)$ & $(0.00009)$ \\
\hline $\mathrm{Cu}$ & & & 0.032 & & & & \\
\hline $\mathrm{Cr}_{2} \mathrm{O}_{3}$ & & & & & & 0.011 & 0.047 \\
\hline \multicolumn{8}{|l|}{$\mathrm{F}$} \\
\hline \multicolumn{8}{|l|}{ In } \\
\hline Total Fe & 65.97 & 66.85 & 90.8 & 59.58 & 65.11 & & \\
\hline $\mathrm{Fe}_{2} \mathrm{O}_{3}$ & & & & & & 7.14 & 8.70 \\
\hline \multicolumn{8}{|l|}{$\mathrm{Pb}$} \\
\hline $\mathrm{MgO}$ & 0.019 & 0.18 & 0.52 & 0.035 & 0.013 & 0.085 & 0.012 \\
\hline $\mathrm{MnO}$ & 0.011 & 0.23 & 0.043 & 0.46 & 0.091 & 0.110 & 0.004 \\
\hline $\mathrm{P}$ & 0.041 & 0.011 & 0.006 & 0.039 & 0.056 & & \\
\hline $\mathrm{P}_{2} \mathrm{O}_{5}$ & & & & & & 0.118 & 0.050 \\
\hline $\mathrm{K}_{2} \mathrm{O}$ & 0.008 & 0.0030 & & 0.039 & 0.0028 & 0.068 & 0.009 \\
\hline $\mathrm{SiO}_{2}$ & 4.17 & 3.71 & 3.7 & 10.14 & 3.87 & 13.43 & 3.79 \\
\hline \multicolumn{8}{|l|}{$\mathrm{Ag}$} \\
\hline $\mathrm{Na}_{2} \mathrm{O}$ & 0.012 & 0.003 & 0.186 & 0.008 & 0.0028 & 0.025 & $(0.007)$ \\
\hline$\underline{S}$ & 0.005 & 0.003 & 0.008 & 0.005 & 0.005 & & \\
\hline$\overline{\mathrm{SO}_{3}}$ & & & & & & 0.63 & 0.21 \\
\hline $\mathrm{TiO}_{2}$ & 0.019 & 0.022 & 0.27 & 0.045 & 0.035 & 1.90 & 2.64 \\
\hline \multicolumn{8}{|l|}{$\mathrm{U}$} \\
\hline $\mathrm{V}_{2} \mathrm{O}_{5}$ & & & & & & 0.028 & 0.072 \\
\hline \multicolumn{8}{|l|}{$\mathrm{WO}_{3}$} \\
\hline \multicolumn{8}{|l|}{$\mathrm{Zn}$} \\
\hline $\mathrm{ZnO}$ & & & & & & 0.0035 & 0.0014 \\
\hline $\mathrm{ZrO}_{2}$ & & & & & & 0.29 & 0.14 \\
\hline \multicolumn{8}{|l|}{ Loss on } \\
\hline Ignition & & & & & & 27.2 & 29.9 \\
\hline Moisture & & & & & & & \\
\hline
\end{tabular}




\section{Ores (Continued)}

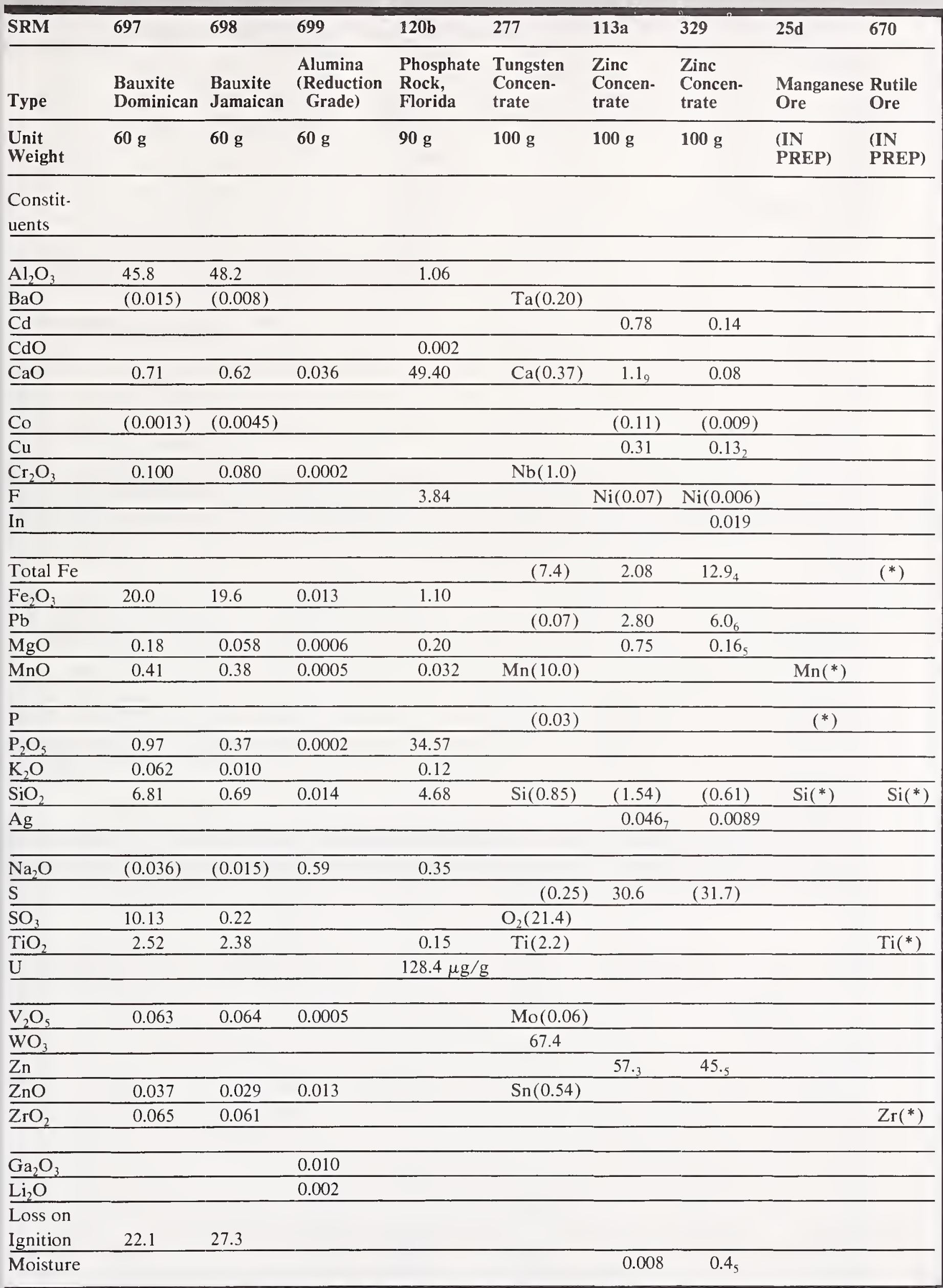

Values in parentheses are not certified, but are given for information only.

*SRM in preparation; values to be certified. 


\section{Rocks, Minerals, and Refractories}

\begin{tabular}{|c|c|c|c|c|c|c|c|c|}
\hline SRM & $1 c$ & $88 a$ & $70 a$ & $99 a$ & $97 a$ & $98 a$ & $81 a$ & $165 a$ \\
\hline Type & $\begin{array}{l}\text { Limestone, } \\
\text { argillaceous }\end{array}$ & $\begin{array}{l}\text { Limestone, } \\
\text { dolomitic }\end{array}$ & $\begin{array}{l}\text { Feldspar, } \\
\text { potash }\end{array}$ & $\begin{array}{l}\text { Feldspar, } \\
\text { soda }\end{array}$ & $\begin{array}{l}\text { Clay, } \\
\text { flint }\end{array}$ & $\begin{array}{l}\text { Clay, } \\
\text { plastic }\end{array}$ & $\begin{array}{l}\text { Glass } \\
\text { sand }\end{array}$ & $\begin{array}{l}\text { Glass sand, } \\
\text { (low iron) }\end{array}$ \\
\hline Unit Weight & $50 \mathrm{~g}$ & $50 \mathrm{~g}$ & $40 \mathrm{~g}$ & $40 \mathrm{~g}$ & $60 \mathrm{~g}$ & $60 \mathrm{~g}$ & $75 \mathrm{~g}$ & $75 \mathrm{~g}$ \\
\hline \multicolumn{9}{|l|}{ Constituents } \\
\hline $\mathrm{Al}_{2} \mathrm{O}_{3}$ & 1.3 & 0.19 & 17.9 & 20.5 & 38.79 & 33.19 & 0.66 & 0.059 \\
\hline $\mathrm{BaO}$ & & & 0.02 & 0.26 & $0.07_{5}$ & 0.03 & & \\
\hline$\overline{\mathrm{CaO}}$ & 50.3 & $30.1_{5}$ & 0.11 & 2.14 & 0.11 & 0.31 & & \\
\hline $\mathrm{Cr}_{2} \mathrm{O}_{3}$ & & & & & 0.03 & 0.03 & $46 \mu \mathrm{g} / \mathrm{g}$ & $(1.1 \mu \mathrm{g} / \mathrm{g})$ \\
\hline $\mathrm{CO}_{2}$ & & 46.6 & & & & & & \\
\hline \multicolumn{9}{|l|}{$\overline{\mathrm{FeO}}$} \\
\hline $\mathrm{Fe}_{2} \mathrm{O}_{3}$ & 0.55 & 0.28 & $0.07_{5}$ & $0.06_{5}$ & 0.45 & 1.34 & 0.082 & 0.012 \\
\hline $\mathrm{Li}_{2} \mathrm{O}$ & & & & & 0.11 & 0.070 & & \\
\hline $\mathrm{MgO}$ & 0.42 & 21.3 & & 0.02 & 0.15 & 0.42 & & \\
\hline $\mathrm{MnO}$ & 0.025 & 0.03 & & & & & & \\
\hline $\mathrm{P}_{2} \mathrm{O}_{5}$ & 0.04 & 0.01 & & 0.02 & 0.36 & 0.11 & & \\
\hline $\mathrm{K}_{2} \mathrm{O}$ & 0.28 & 0.12 & 11.8 & 5.2 & 0.50 & 1.04 & & \\
\hline $\mathrm{Rb}_{2} \mathrm{O}$ & & & 0.06 & & & & & \\
\hline$\overline{\mathrm{SiO}_{2}}$ & 6.84 & 1.20 & 67.1 & 65.2 & 43.67 & 48.94 & & \\
\hline $\mathrm{Na}_{2} \mathrm{O}$ & 0.02 & 0.01 & $2.5_{5}$ & 6.2 & 0.037 & 0.082 & & \\
\hline $\mathrm{SrO}$ & 0.030 & 0.010 & & & 0.18 & 0.039 & & \\
\hline $\mathrm{TiO}_{2}$ & 0.07 & 0.02 & 0.01 & 0.007 & 1.90 & 1.61 & 0.12 & 0.011 \\
\hline $\mathrm{ZrO}_{2}$ & & & & & 0.063 & 0.042 & 0.034 & 0.006 \\
\hline $\begin{array}{l}\text { Loss on } \\
\text { Ignition }\end{array}$ & 39.9 & 46.7 & 0.40 & 0.26 & 13.32 & 12.44 & & \\
\hline
\end{tabular}

\begin{tabular}{lllllll}
\hline SRM & 154b & 278 & 688 & $76 \mathrm{a}$ & $77 \mathrm{a}$ & $78 \mathrm{a}$ \\
\hline Type & $\begin{array}{l}\text { Titanium } \\
\text { dioxide }\end{array}$ & $\begin{array}{l}\text { Obsidian } \\
\text { rock }\end{array}$ & $\begin{array}{l}\text { Basalt } \\
\text { rock }\end{array}$ & $\begin{array}{l}\text { Burnt } \\
\text { Refractory } \\
\left(\mathrm{Al}_{2} \mathrm{O}_{3}-40 \%\right)\end{array}$ & $\begin{array}{l}\text { Burnt } \\
\text { Refractory } \\
\left(\mathrm{Al}_{2} \mathrm{O}_{3}-60 \%\right)\end{array}$ & $\begin{array}{l}\text { Burnt } \\
\text { Refractory } \\
\left(\mathrm{Al}_{2} \mathrm{O}_{3}-70 \%\right)\end{array}$ \\
\hline Unit Weight & $90 \mathrm{~g}$ & $35 \mathrm{~g}$ & $\mathbf{6 0 \mathrm { g }}$ & $\mathbf{7 5} \mathrm{g}$ & $75 \mathrm{~g}$ & $\mathbf{7 5 \mathrm { g }}$ \\
\hline
\end{tabular}

Constituents

\begin{tabular}{|c|c|c|c|c|c|}
\hline $\mathrm{Al}_{2} \mathrm{O}_{3}$ & 14.15 & 17.36 & 38.7 & 60.2 & 71.7 \\
\hline \multicolumn{6}{|l|}{$\overrightarrow{\mathrm{BaO}}$} \\
\hline $\mathrm{CaO}$ & 0.983 & & 0.22 & 0.05 & 0.11 \\
\hline \multicolumn{6}{|l|}{$\mathrm{Cr}_{2} \mathrm{O}_{3}$} \\
\hline \multicolumn{6}{|l|}{$\mathrm{CO}_{2}$} \\
\hline $\mathrm{FeO}$ & & 7.64 & & & \\
\hline$\overline{\mathrm{Fe}_{2} \mathrm{O}_{3}}$ & 2.04 & 10.35 & $1.6_{0}$ & $1.0_{0}$ & 1.2 \\
\hline $\mathrm{Li}_{2} \mathrm{O}$ & & & 0.042 & $0.2_{5}$ & 0.12 \\
\hline$\overline{\mathrm{MgO}}$ & & & 0.52 & 0.38 & 0.70 \\
\hline $\mathrm{MnO}$ & 0.052 & 0.167 & & & \\
\hline
\end{tabular}




\begin{tabular}{|c|c|c|c|c|c|c|}
\hline SRM & $154 \mathrm{~b}$ & 278 & 688 & $76 a$ & $77 a$ & $78 a$ \\
\hline Type & $\begin{array}{l}\text { Titanium } \\
\text { dioxide }\end{array}$ & $\begin{array}{l}\text { Obsidian } \\
\text { rock }\end{array}$ & $\begin{array}{l}\text { Basalt } \\
\text { rock }\end{array}$ & $\begin{array}{l}\text { Burnt } \\
\text { Refractory } \\
\left(\mathrm{Al}_{2} \mathrm{O}_{3}-40 \%\right)\end{array}$ & $\begin{array}{l}\text { Burnt } \\
\text { Refractory } \\
\left(\mathrm{Al}_{2} \mathrm{O}_{3}-60 \%\right)\end{array}$ & $\begin{array}{l}\text { Burnt } \\
\text { Refractory } \\
\left(\mathrm{Al}_{2} \mathrm{O}_{3}-70 \%\right)\end{array}$ \\
\hline Unit Weight & $90 \mathrm{~g}$ & $35 \mathrm{~g}$ & $60 \mathrm{~g}$ & $75 \mathrm{~g}$ & $75 \mathrm{~g}$ & $75 \mathrm{~g}$ \\
\hline \multicolumn{7}{|l|}{ Constituents } \\
\hline$\underline{\mathrm{P}_{2} \mathrm{O}_{5}}$ & & 0.036 & 0.134 & $0.12_{0}$ & 0.092 & 1.3 \\
\hline$\underline{\mathrm{K}_{2} \mathrm{O}}$ & & 4.16 & 0.187 & 1.33 & $0.09_{0}$ & 1.22 \\
\hline \multicolumn{7}{|l|}{$\mathrm{Rb}_{2} \mathrm{O}$} \\
\hline $\mathrm{SiO}_{2}$ & & 73.05 & 48.4 & 54.9 & 35.0 & 19.4 \\
\hline$\underline{\mathrm{Na}_{2} \mathrm{O}}$ & & 4.84 & 2.15 & 0.07 & 0.037 & 0.078 \\
\hline SrO & & & & 0.037 & 0.009 & 0.25 \\
\hline $\mathrm{TiO}_{2}$ & 99.74 & 0.245 & 1.17 & $2.0_{3}$ & $2.6_{6}$ & $3.2_{2}$ \\
\hline $\mathrm{ZrO}_{2}$ & & & & 0.15 & 0.21 & 0.31 \\
\hline $\begin{array}{l}\text { Loss on } \\
\text { Ignition }\end{array}$ & & & & $(0.34)$ & $(0.22)$ & $(0.42)$ \\
\hline
\end{tabular}

\begin{tabular}{|c|c|c|c|}
\hline SRM & $103 a$ & 198 & 199 \\
\hline Type & $\begin{array}{l}\text { Chrome } \\
\text { Refractory }\end{array}$ & $\begin{array}{l}\text { Silica } \\
\text { Refractory }\end{array}$ & $\begin{array}{l}\text { Silica } \\
\text { Refractory }\end{array}$ \\
\hline Unit Weight & $60 \mathrm{~g}$ & $45 \mathrm{~g}$ & $45 \mathrm{~g}$ \\
\hline \multicolumn{4}{|l|}{ Constituents } \\
\hline $\mathrm{Al}_{2} \mathrm{O}_{3}$ & 29.96 & 0.16 & 0.48 \\
\hline \multicolumn{4}{|l|}{$\mathrm{BaO}$} \\
\hline $\mathrm{CaO}$ & 0.69 & 2.71 & 2.41 \\
\hline $\mathrm{Cr}_{2} \mathrm{O}_{3}$ & 32.06 & & \\
\hline \multicolumn{4}{|l|}{$\mathrm{CO}_{2}$} \\
\hline$\overline{\mathrm{FeO}}$ & 12.43 & & \\
\hline$\overline{\mathrm{Fe}_{2} \mathrm{O}_{3}}$ & & 0.66 & 0.74 \\
\hline $\mathrm{Li}_{2} \mathrm{O}$ & & 0.001 & 0.002 \\
\hline$\overline{\mathrm{MgO}}$ & 18.54 & 0.07 & 0.13 \\
\hline $\mathrm{MnO}$ & 0.11 & 0.008 & 0.007 \\
\hline$\overline{\mathrm{P}_{2} \mathrm{O}_{5}}$ & 0.01 & 0.022 & 0.015 \\
\hline $\mathrm{K}_{2} \mathrm{O}$ & & 0.017 & 0.094 \\
\hline \multicolumn{4}{|l|}{$\mathrm{Rb}_{2} \mathrm{O}$} \\
\hline $\mathrm{SiO}_{2}$ & 4.63 & & \\
\hline $\mathrm{Na}_{2} \mathrm{O}$ & & 0.012 & 0.015 \\
\hline \multicolumn{4}{|l|}{$\overline{\mathrm{SrO}}$} \\
\hline $\mathrm{TiO}_{2}$ & 0.22 & 0.02 & 0.06 \\
\hline $\mathrm{ZrO}_{2}$ & 0.01 & $<0.01$ & 0.01 \\
\hline \multicolumn{4}{|l|}{ Loss on } \\
\hline Ignition & & 0.21 & 0.17 \\
\hline
\end{tabular}

Values in parentheses are not certified, but are given for information only. 


\section{Glasses}

\begin{tabular}{|c|c|c|c|c|c|c|c|c|}
\hline SRM & 89 & 91 & 92 & 93a & 620 & 621 & 1830 & 1831 \\
\hline Tyре & $\begin{array}{l}\text { Lead- } \\
\text { Barium }\end{array}$ & Opal & $\begin{array}{l}\text { Low- } \\
\text { Boron }\end{array}$ & $\begin{array}{l}\text { High- } \\
\text { Boron }\end{array}$ & $\begin{array}{l}\text { Soda-Lime, } \\
\text { Flat }\end{array}$ & $\begin{array}{l}\text { Soda-Lime, } \\
\text { Container }\end{array}$ & $\begin{array}{l}\text { Soda-Lime, } \\
\text { Float }\end{array}$ & $\begin{array}{l}\text { Soda-Lime } \\
\text { Sheet }\end{array}$ \\
\hline Unit Size & $45 \mathrm{~g}$ & $45 \mathrm{~g}$ & $45 \mathrm{~g}$ & $\begin{array}{l}\text { Wafer } \\
32 \mathrm{~mm} \mathrm{D} \\
\times 6 \mathrm{~mm}\end{array}$ & $\begin{array}{l}3 \text { platelets } \\
35 \times 35 \\
\times 3 \mathrm{~mm}\end{array}$ & $\begin{array}{l}3 \text { disks } \\
38 \mathrm{~mm} \mathrm{D} \\
\times 5 \mathrm{~mm}\end{array}$ & $\begin{array}{l}3 \text { platelets } \\
38 \times 38 \\
\times 6 \mathrm{~mm}\end{array}$ & $\begin{array}{l}3 \text { platelets } \\
37 \times 37 \\
\times 3 \mathrm{~mm}\end{array}$ \\
\hline Constituent & \multicolumn{8}{|c|}{ Chemical Composition (Nominal Weight Percent) } \\
\hline $\mathrm{SiO}_{2}$ & 65.35 & 67.50 & $(75.0)$ & 80.8 & 72.8 & 71.13 & 73.07 & 73.08 \\
\hline$\underline{\mathrm{PbO}}$ & 17.50 & 0.10 & & & & & & \\
\hline $\mathrm{Al}_{2} \mathrm{O}_{3}$ & 0.18 & 6.01 & & 2.28 & 1.80 & 2.76 & 0.12 & 1.21 \\
\hline $\mathrm{Fe}_{2} \mathrm{O}_{3}$ & 0.049 & 0.079 & & 0.028 & 0.043 & 0.040 & 0.121 & 0.087 \\
\hline $\mathrm{ZnO}$ & & 0.08 & $(0.2)$ & & & & & \\
\hline $\mathrm{MnO}$ & 0.088 & $(0.008)$ & & & & & & \\
\hline $\mathrm{TiO}_{2}$ & 0.01 & 0.019 & & 0.014 & 0.018 & 0.014 & 0.011 & 0.019 \\
\hline $\mathrm{ZrO}_{2}$ & 0.005 & 0.009 & & 0.042 & & 0.007 & & \\
\hline $\mathrm{CaO}$ & 0.21 & 10.49 & $(8.3)$ & 0.01 & 7.11 & 10.71 & 8.56 & 8.20 \\
\hline $\mathrm{BaO}$ & 1.40 & & & & & 0.12 & & \\
\hline$\overline{\mathrm{MgO}}$ & 0.03 & $(0.008)$ & $(0.1)$ & 0.005 & 3.69 & 0.27 & 3.90 & 3.51 \\
\hline$\underline{\mathrm{K}_{2} \mathrm{O}}$ & 8.40 & 3.24 & $(0.6)$ & 0.014 & 0.41 & 2.01 & 0.04 & 0.33 \\
\hline $\mathrm{Na}_{2} \mathrm{O}$ & 5.70 & 8.47 & $(13.1)$ & 3.98 & 14.39 & 12.74 & 13.75 & 13.32 \\
\hline $\mathrm{B}_{2} \mathrm{O}_{3}$ & & & 0.70 & 12.56 & & & & \\
\hline$\underline{\mathrm{P}_{2} \mathrm{O}_{5}}$ & 0.23 & 0.023 & & & & & & \\
\hline $\mathrm{As}_{2} \mathrm{O}_{5}$ & 0.36 & 0.10 & & & & & & \\
\hline $\mathrm{As}_{2} \mathrm{O}_{3}$ & 0.03 & 0.09 & & & 0.056 & 0.030 & & \\
\hline $\mathrm{SO}_{3}$ & 0.03 & & & & 0.28 & 0.13 & 0.26 & 0.25 \\
\hline $\mathrm{R}_{2} \mathrm{O}_{3}$ & & & $(1.5)$ & & & & & \\
\hline $\mathrm{Cl}$ & . 0.05 & 0.015 & & 0.06 & & & & \\
\hline F & & 5.73 & & & & & & \\
\hline Loss on & & & & & & & & \\
\hline Ignition & 0.32 & & $(0.42)$ & & & & & \\
\hline
\end{tabular}

Values in parentheses are not certified, but are given for information only.

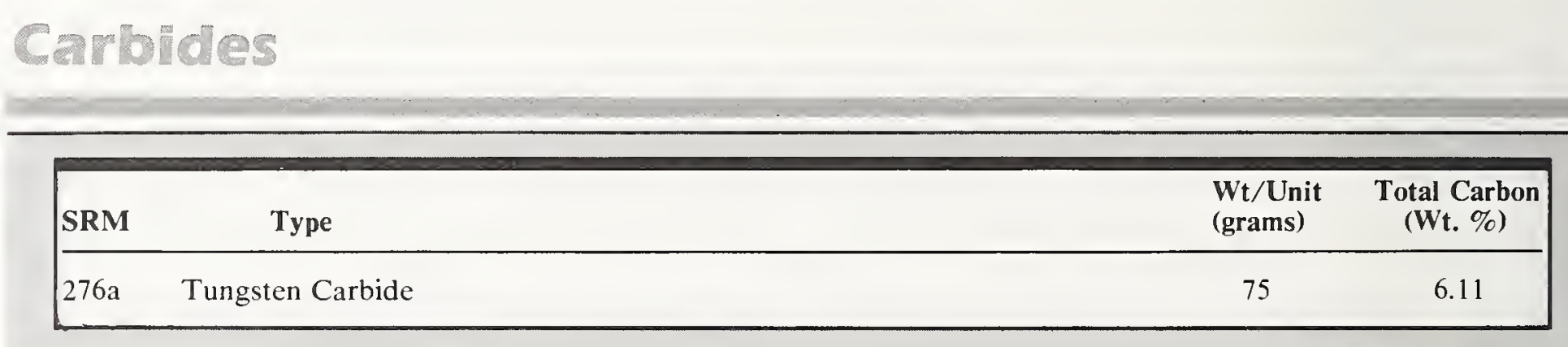


These SRM's are furnished for $\mathrm{x}$-ray spectroscopic analysis and for chemical analysis of cements and related materials. Because these SRM's are hygroscopic, each unit consists of three sealed vials each containing approximately $5 \mathrm{~g}$ of material.

\begin{tabular}{|c|c|c|c|c|c|c|c|c|c|}
\hline SRM & 633 & 634 & 635 & 636 & 637 & 638 & 639 & 1880 & 1881 \\
\hline Type & RED & GOLD & BLUE & YELLOW & PINK & GREEN & CLEAR & BLACK & WHITE \\
\hline Unit Weight & $15 \mathrm{~g}$ & $15 \mathrm{~g}$ & $15 \mathrm{~g}$ & $15 \mathrm{~g}$ & $15 \mathrm{~g}$ & $15 \mathrm{~g}$ & $15 \mathrm{~g}$ & $15 \mathrm{~g}$ & $15 \mathrm{~g}$ \\
\hline Constituent & \multicolumn{9}{|c|}{ Chemical Composition (Nominal Weight Percent) } \\
\hline $\mathrm{CaO}$ & $64.5_{0}$ & $62.5_{8}$ & $59.8_{3}$ & $63.5_{4}$ & $66.0_{4}$ & $62.0_{9}$ & $65.7_{6}$ & 63.13 & 58.67 \\
\hline $\mathrm{SiO}_{2}$ & $21.8_{8}$ & $20.7_{3}$ & $18.4_{1}$ & $23.2_{2}$ & $23.0_{7}$ & $21.4_{8}$ & $21.6_{1}$ & 19.82 & 22.25 \\
\hline $\mathrm{Al}_{2} \mathrm{O}_{3}$ & $3.7_{8}$ & $5.2_{1}$ & $6.2_{9}$ & $3.0_{2}$ & $3.2_{8}$ & $4.4_{5}$ & $4.2_{8}$ & 5.02 & 4.19 \\
\hline $\mathrm{Fe}_{2} \mathrm{O}_{3}$ & 4.20 & 2.84 & 2.61 & 1.61 & 1.80 & 3.55 & 2.40 & 2.91 & 4.68 \\
\hline $\mathrm{SO}_{3}$ & $2.2_{0}$ & $2.2_{1}$ & $7.0_{7}$ & $2.3_{1}$ & $2.3_{8}$ & $2.3_{4}$ & $2.4_{8}$ & 3.37 & 3.65 \\
\hline $\mathrm{MgO}$ & $1.0_{4}$ & $3.3_{0}$ & $1.2_{3}$ & $3.9_{5}$ & $0.6_{7}$ & $3.8_{3}$ & $1.2_{6}$ & 2.69 & 2.62 \\
\hline $\mathrm{K}_{2} \mathrm{O}$ & 0.17 & 0.42 & 0.45 & 0.59 & 0.25 & 0.59 & 0.06 & 0.91 & 1.17 \\
\hline $\mathrm{TiO}_{2}$ & 0.24 & 0.29 & 0.32 & 0.18 & 0.21 & 0.25 & 0.32 & 0.23 & 0.23 \\
\hline $\mathrm{Na}_{2} \mathrm{O}$ & 0.64 & 0.15 & 0.07 & 0.11 & 0.15 & 0.13 & 0.65 & 0.28 & 0.04 \\
\hline $\mathrm{SrO}$ & 0.31 & 0.12 & 0.21 & 0.04 & 0.09 & 0.07 & 0.15 & 0.06 & 0.11 \\
\hline$\overline{\mathrm{P}_{2} \mathrm{O}_{5}}$ & 0.24 & 0.10 & 0.17 & 0.08 & 0.24 & 0.06 & 0.08 & 0.29 & 0.09 \\
\hline $\mathrm{Mn}_{2} \mathrm{O}_{3}$ & 0.04 & 0.28 & 0.09 & 0.12 & 0.06 & 0.05 & 0.08 & 0.08 & 0.26 \\
\hline $\mathrm{F}$ & 0.08 & 0.08 & 0.04 & 0.06 & 0.04 & 0.04 & 0.02 & 0.10 & 0.09 \\
\hline $\mathrm{ZnO}$ & 0.01 & 0.02 & 0.01 & 0.03 & 0.01 & 0.10 & 0.01 & 0.01 & 0.01 \\
\hline $\mathrm{Cr}_{2} \mathrm{O}_{3}$ & 0.01 & 0.08 & 0.01 & 0.01 & 0.01 & 0.01 & 0.01 & & \\
\hline Ignition loss & $0.7_{5}$ & $1.6_{2}$ & $3.2_{4}$ & $1.1_{6}$ & $1.6_{9}$ & $0.9_{5}$ & $1.0_{0}$ & 1.38 & 2.01 \\
\hline Total & 100.06 & 100.00 & 100.03 & 100.00 & 99.97 & 99.97 & 100.16 & 100.30 & 100.07 \\
\hline
\end{tabular}
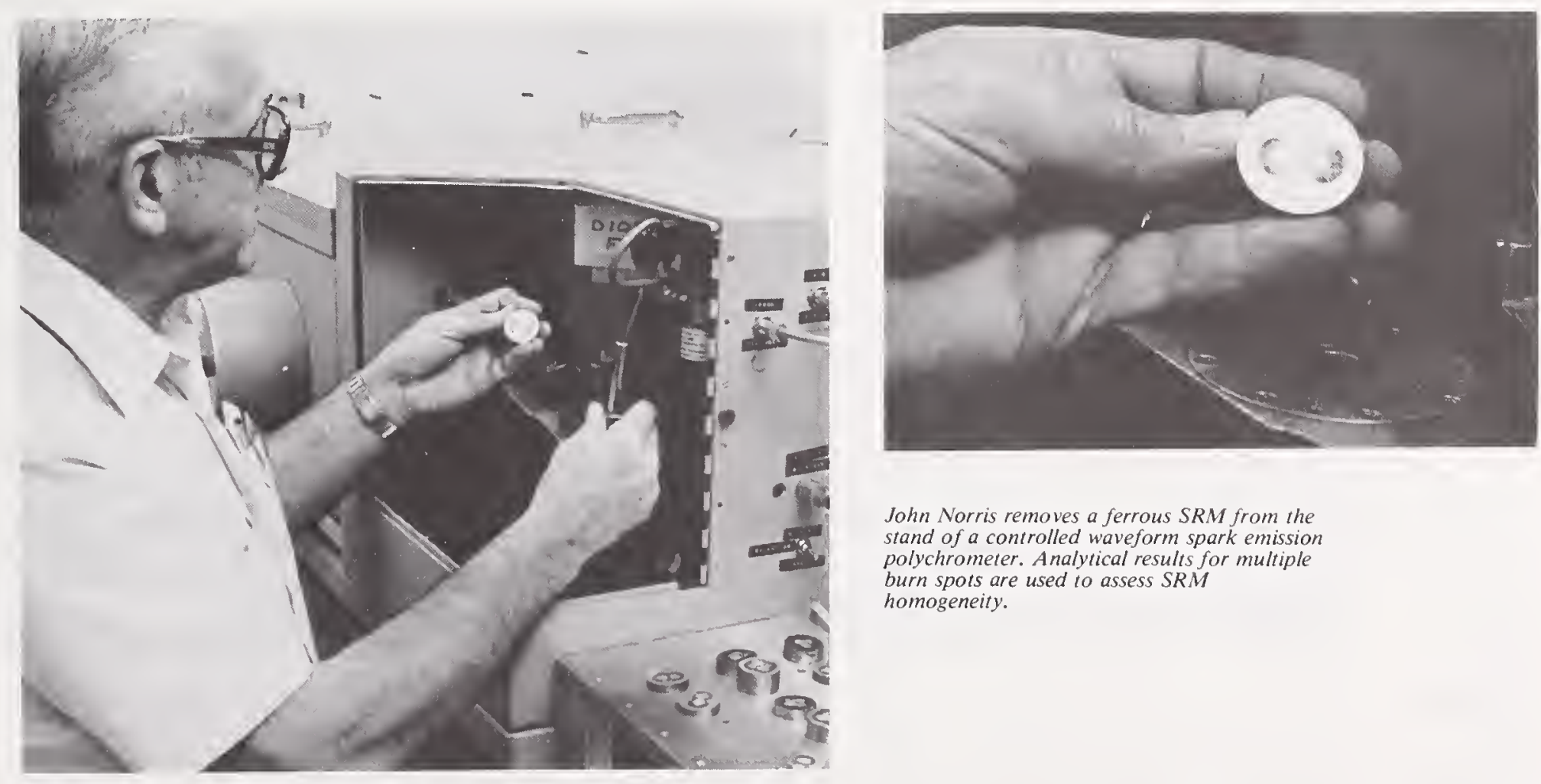

John Norris removes a ferrous SRM from the stand of a controlled waveform spark emission polychrometer. Analytical results for multiple burn spots are used to assess SRM homogeneity. 


\section{Trace Elements}

The SRM's listed below were designed for trace chemical analysis, specifically for calibrating instruments and evaluating analytical techniques and procedures used to determine trace elements in various inorganic matrices. In addition many SRM's certified for chemical composition have one or more constituents certified at or below the $100 \mu \mathrm{g} / \mathrm{g}$ level. Some SRM's in the following categories may be of use in trace analytical work:

Steels (pages 19-38); High-Purity Metals (page 52); Nonferrous Alloys (pages 39-51); Environmental Standards (page 59); and Biological Standards (page 57).

\begin{tabular}{|c|c|c|c|c|c|}
\hline SRM & 607 & $610-611$ & $612-613$ & 614-615 & 616-617 \\
\hline Type & $\begin{array}{l}\text { Trace Elements } \\
\text { in Potassium } \\
\text { Feldspar }\end{array}$ & $\begin{array}{l}\text { Trace Elements } \\
\text { in Glass }\end{array}$ & $\begin{array}{l}\text { Trace Elements } \\
\text { in Glass }\end{array}$ & $\begin{array}{l}\text { Trace Elements } \\
\text { in Glass }\end{array}$ & $\begin{array}{l}\text { Trace Elements } \\
\text { in Glass }\end{array}$ \\
\hline $\begin{array}{l}\text { Concentration } \\
\text { Range }\end{array}$ & & $500 \mathrm{ppm}$ & $50 \mathrm{ppm}$ & $1 \mathrm{ppm}$ & $0.02 \mathrm{ppm}$ \\
\hline $\begin{array}{l}\text { Wafer } \\
\text { Thickness }\end{array}$ & & $\begin{array}{l}6103 \mathrm{~mm} \\
6111 \mathrm{~mm}\end{array}$ & $\begin{array}{l}6123 \mathrm{~mm} \\
6131 \mathrm{~mm}\end{array}$ & $\begin{array}{l}6143 \mathrm{~mm} \\
6151 \mathrm{~mm}\end{array}$ & $\begin{array}{l}6163 \mathrm{~mm} \\
6171 \mathrm{~mm}\end{array}$ \\
\hline Unit of Issue & $5 \mathrm{~g}$ & 6 Wafers & 6 Wafers & 6 Wafers & 6 Wafers \\
\hline Element & & Nominal & oncentrations ( $\mathrm{ppm}$ & & \\
\hline Antimony & & & & $(1.06)$ & $(0.078)$ \\
\hline Barium & & & (41) & & \\
\hline Boron & & (351) & (32) & $(1.30)$ & $(0.20)$ \\
\hline Cadmium & & & & $(0.55)$ & \\
\hline Cerium & & & $(39)$ & & \\
\hline Cobalt & & $(390)$ & $(35.5)$ & $(0.73)$ & \\
\hline Copper & & (444) & $(37.7)$ & 1.37 & $(0.80)$ \\
\hline Dysprosium & & & (35) & & \\
\hline Erbium & & & (39) & & \\
\hline Europium & & & (36) & $(0.99)$ & \\
\hline Gadolinium & & & (39) & & \\
\hline Gallium & & & & (1.3) & $(0.23)$ \\
\hline Gold & & (25) & (5) & $(0.5)$ & $(0.18)$ \\
\hline Iron & & 458 & 51 & (13.3) & (11) \\
\hline Lanthanum & & & (36) & $(0.83)$ & $(0.034)$ \\
\hline Lead & & 426 & 38.57 & 2.32 & 1.85 \\
\hline Manganese & & 485 & $(39.6)$ & & \\
\hline Neodymium & & & $(36)$ & & \\
\hline Nickel & & 458.7 & 38.8 & $(0.95)$ & \\
\hline Potassium & & $(461)$ & $(64)$ & 30 & 29 \\
\hline Rubidium & 523.90 & 425.7 & 31.4 & 0.855 & 0.100 \\
\hline Samarium & & & (39) & & \\
\hline Scandium & & & & $(0.59)$ & $(0.026)$ \\
\hline Silver & & (254) & 22.0 & 0.42 & \\
\hline Strontium & 65.485 & 515.5 & 78.4 & 45.8 & 41.72 \\
\hline
\end{tabular}




\section{Trace Elements (Continued)}

\begin{tabular}{llcccc}
\hline SRM & $\mathbf{6 0 7}$ & $\mathbf{6 1 0 - 6 1 1}$ & $\mathbf{6 1 2 - 6 1 3}$ & $\mathbf{6 1 4 - 6 1 5}$ & $\mathbf{6 1 6 - 6 1 7}$ \\
\hline $\begin{array}{l}\text { Trace Elements } \\
\text { in Potassium } \\
\text { Type }\end{array}$ & $\begin{array}{l}\text { Trace Elements } \\
\text { in Glass }\end{array}$ & $\begin{array}{l}\text { Trace Elements } \\
\text { in Glass }\end{array}$ & $\begin{array}{l}\text { Trace Elements } \\
\text { in Glass }\end{array}$ & $\begin{array}{l}\text { Trace Elements } \\
\text { in Glass }\end{array}$ \\
\hline Thallium & & $(61.8)$ & $(15.7)$ & $(0.269)$ & $(0.0082)$ \\
\hline Thorium & 457.2 & 37.79 & 0.748 & 0.0252 \\
\hline Titanium & $(437)$ & $(50.1)$ & $(3.1)$ & 0.823 & 0.0721 \\
\hline Uranium & 461.5 & 37.38 & $(2.5)$ \\
\hline Ytterbium & & $(42)$ & & \\
\hline
\end{tabular}

In addition to the elements listed above, the glass SRM's contain the following 25 elements: $\mathrm{As}, \mathrm{Be}, \mathrm{Bi}, \mathrm{Cs}$, $\mathrm{Cl}, \mathrm{F}, \mathrm{Ge}, \mathrm{Hf}, \mathrm{Hg}, \mathrm{Li}, \mathrm{Lu}, \mathrm{Mg}, \mathrm{Nb}, \mathrm{P}, \mathrm{Pr}, \mathrm{Se}, \mathrm{S}, \mathrm{Te}, \mathrm{Tb}, \mathrm{Tm}, \mathrm{Sn}, \mathrm{W}, \mathrm{V}, \mathrm{Y}$, and $\mathrm{Zr}$.

Note: Glass-Nominal Composition: $72 \% \mathrm{SiO}_{2}, 12 \% \mathrm{CaO}, 14 \% \mathrm{Na}_{2} \mathrm{O}$, and $2 \% \mathrm{Al}_{2} \mathrm{O}_{3}$.

Values in parentheses are not certified, but are given for information only.

\section{Nuclear Materials}

\section{Special Nuclear Materials}

These SRM's are available to Department of Energy contractors, Nuclear Regulatory Commission, or State Licensees, and foreign governments that have entered into an agreement of cooperation with the U.S. Government regarding the use of these materials. Purchase orders and requests for information regarding ordering procedures, availability, and shipment of these SRM's should be directed to:

NBS Special Nuclear Standard Reference Materials

U.S. Department of Energy

New Brunswick Laboratory, D.350

9800 South Cass Avenue

Argonne, IL 60439

(312) 272-2485

FTS: 972.2485

\begin{tabular}{|c|c|c|c|c|}
\hline \multicolumn{5}{|c|}{ Plutonium Assay } \\
\hline SRM & Identification (Batch Name) & $\begin{array}{l}\text { Constituent } \\
\text { Certified }\end{array}$ & $\begin{array}{l}\text { Element Weight* } \\
(\mathrm{g})\end{array}$ & (Weight Percent) \\
\hline$\underline{945}$ & Plutonium Metal, standard matrix & Impurities & 5 & 99.9 \\
\hline$\overline{949 f}$ & Plutonium Metal Assay & Plutonium Content & 0.5 & 99.99 \\
\hline
\end{tabular}

\section{Plutonium Isotopic}

\begin{tabular}{|c|c|c|c|c|c|c|c|c|}
\hline \multirow[b]{2}{*}{ SRM } & \multirow[b]{2}{*}{ Identification (Batch Name) } & \multirow{2}{*}{$\begin{array}{l}\text { Element } \\
\text { Weight } \\
\text { (g) }\end{array}$} & \multicolumn{6}{|c|}{ Certified Isotopes (Atom Percent) } \\
\hline & & & ${ }^{238} \mathrm{Pu}$ & ${ }^{239} \mathrm{Pu}$ & ${ }^{240} \mathrm{Pu}$ & ${ }^{241} \mathrm{Pu}$ & ${ }^{242} \mathrm{Pu}$ & ${ }^{244} \mathrm{Pu}$ \\
\hline 946 & Plutonium Sulfate Tetrahydrate & 0.25 & 0.232 & 84.464 & 12.253 & 2.477 & 0.574 & \\
\hline$\overline{947}$ & Plutonium Sulfate Tetrahydrate & 0.25 & 0.278 & 77.089 & 18.610 & 2.821 & 1.202 & \\
\hline 948 & Plutonium Sulfate Tetrahydrate & 0.25 & 0.010 & 91.736 & 7.922 & 0.299 & 0.0330 & \\
\hline 996 & Plutonium-Spike & 0.001 & 0.005 & 0.034 & 0.677 & 0.092 & 1.325 & 97.867 \\
\hline
\end{tabular}




\section{Uranium Assay}

\begin{tabular}{|llccc|}
\hline SRM & Identification (Batch Name) & $\begin{array}{c}\text { Constituent } \\
\text { Certified }\end{array}$ & $\begin{array}{c}\text { Element Weight } \\
\text { (g) }\end{array}$ & (Weight Percent) \\
\hline $950 \mathrm{~b}$ & Uranium Oxide & $\mathrm{U}_{3} \mathrm{O}_{8}$ & 25 & 99.968 \\
\hline 960 & Uranium Metal & $\mathrm{U}$ & 26 & 99.975 \\
\hline 993 & Uranium-235 Spike (solution) & $\mathrm{U}-235$ & 0.1 & 99.8195 \\
\hline 995 & Uranium-233 Spike (solution) & $\mathrm{U}-235$ & 0.005 & 99.9245 \\
\hline 969 & $\begin{array}{c}\text { Uranium Oxide-IN PREP } \\
\text { (for NDA measurements) }\end{array}$ & & $\begin{array}{c}\text { Set of 5 enrichments } \\
\text { (for }\end{array}$ & $0.3,0.7,2.0,3.0,4.5 \%$ \\
\hline
\end{tabular}

\section{Uranium Isotopic}

\begin{tabular}{|c|c|c|c|c|c|c|}
\hline \multirow[b]{2}{*}{ SRM } & \multirow[b]{2}{*}{ Uranium Oxide $\left(\mathrm{U}_{3} \mathrm{O}_{8}\right)$} & \multirow{2}{*}{$\begin{array}{c}\text { Wt } \\
\text { (grams) }\end{array}$} & \multicolumn{4}{|c|}{ Certified Isotopes (Atom Percent) } \\
\hline & & & ${ }^{234} \mathrm{U}$ & ${ }^{235} \mathrm{U}$ & ${ }^{234} \mathrm{U}$ & ${ }^{238} \mathrm{U}$ \\
\hline $\mathrm{U}-0002$ & Depleted & 1.0 & 0.00016 & 0.01755 & $<0.00001$ & 99.9823 \\
\hline $\mathrm{U}-005 \mathrm{a}$ & Depleted-IN PREP & & & & & \\
\hline $\mathrm{U}-010$ & Enriched & 1.0 & 0.00541 & 1.0037 & 0.00681 & 98.984 \\
\hline $\mathrm{U}-015$ & Enriched & 1.0 & 0.00850 & 1.5323 & 0.0164 & 98.443 \\
\hline $\mathrm{U}-020 \mathrm{a}$ & Enriched-IN PREP & & & & & \\
\hline $\mathrm{U}-030 \mathrm{a}$ & Enriched-IN PREP & & & & & \\
\hline$\underline{\mathrm{U}-050}$ & Enriched & 1.0 & 0.0279 & 5.010 & 0.0480 & 94.915 \\
\hline $\mathrm{U}-100$ & Enriched & 1.0 & 0.0676 & 10.190 & 0.0379 & 89.704 \\
\hline$\underline{\mathrm{U}-150}$ & Enriched & 1.0 & 0.0993 & 15.307 & 0.0660 & 84.528 \\
\hline $\mathrm{U}-200$ & Enriched & 1.0 & 0.1246 & 20.013 & 0.2116 & 79.651 \\
\hline $\mathrm{U}-350$ & Enriched & 1.0 & 0.2498 & 35.190 & 0.1673 & 64.393 \\
\hline $\mathrm{U}-500$ & Enriched & 1.0 & 0.5181 & 49.696 & 0.0755 & 49.711 \\
\hline $\mathrm{U}-750$ & Enriched & 1.0 & 0.5923 & 75.357 & 0.2499 & 23.801 \\
\hline $\mathrm{U}-800$ & Enriched. & 1.0 & 0.6563 & 80.279 & 0.2445 & 18.820 \\
\hline $\mathrm{U}-850$ & Enriched & 1.0 & 0.6437 & 85.137 & 0.3704 & 13.848 \\
\hline $\mathrm{U}-900$ & Enriched & 1.0 & 0.7777 & 90.196 & 0.3327 & 8.693 \\
\hline $\mathrm{U}-930$ & Enriched & 1.0 & 1.0812 & 93.336 & 0.2027 & 5.380 \\
\hline$\overline{\mathrm{U}-970}$ & Enriched & 1.0 & 1.6653 & 97.663 & 0.1491 & 0.5229 \\
\hline
\end{tabular}




\section{Radiation Dosimetry}

This SRM is a cobalt-in-aluminum alloy (wire form) to serve as a neutron density monitor standard. Accurate determination of thermal neutron densities is essential in irradiation tests to obtain a basis for comparison of densities within and among reactors. SRM 953 is intended for use in providing data for the design of reactors, understanding the mechanisms of radiation damage, and for use in using neutron activation analysis. The wire is $0.5 \mathrm{~mm}$ in diameter and 1 meter long.

\begin{tabular}{|c|c|c|}
\hline SRM & Identification (Batch Name) & $\begin{array}{c}\text { Cobalt Content } \\
\text { (Weight Percent) }\end{array}$ \\
\hline 953 & Neutron density monitor wire (Co in $\mathrm{Al}$ ) & 0.116 \\
\hline 8505 & Vanadium in Crude Oil-IN PREP & \\
\hline
\end{tabular}

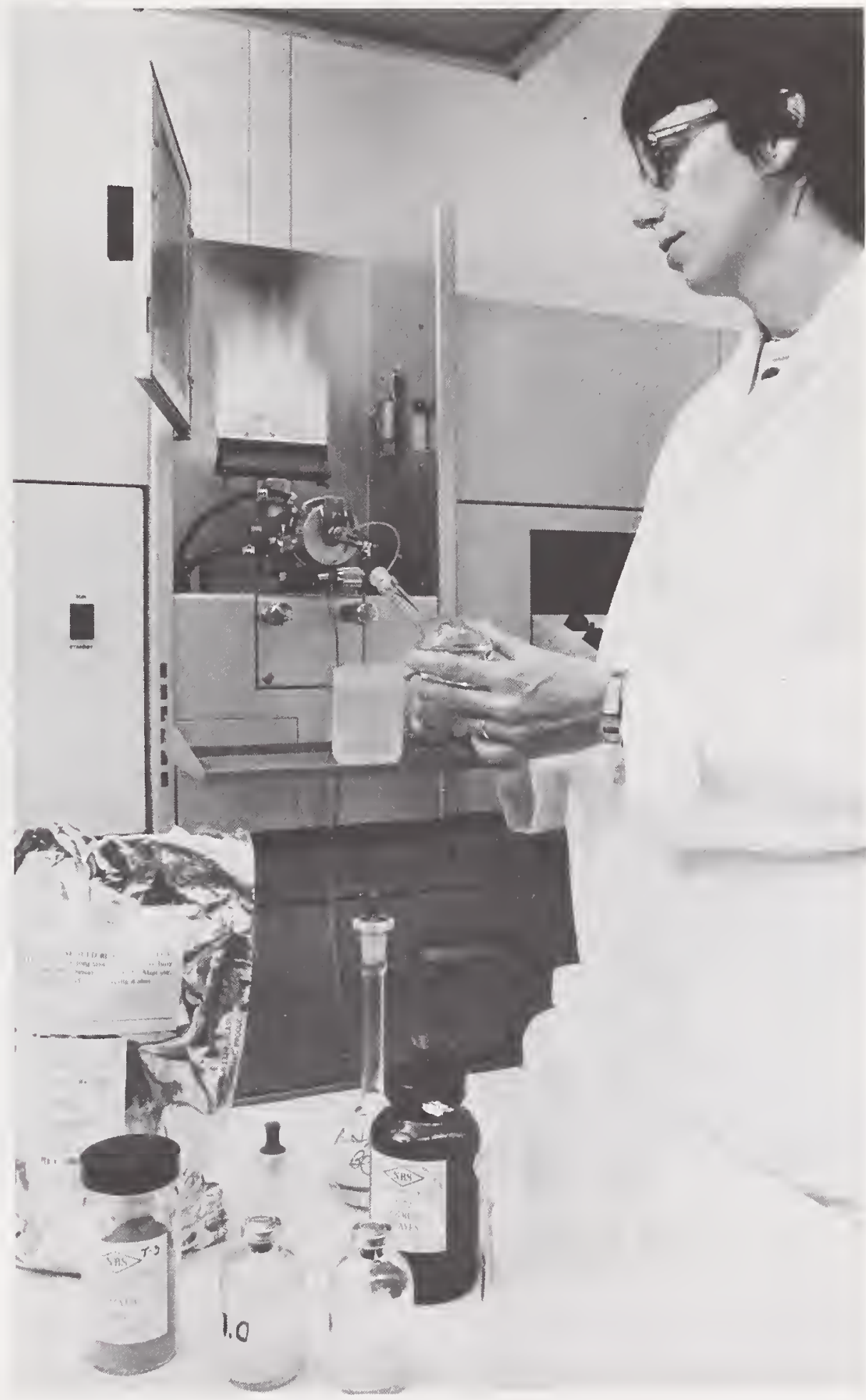

Therese Butler aspirates a solution of an SRM into the flame atomic absorption $(A A)$ spectrometer. Major and minor elements are determined in a variety of SRMs using flame $A A$, while trace elements are determined using electrothermal atomization $A A$.

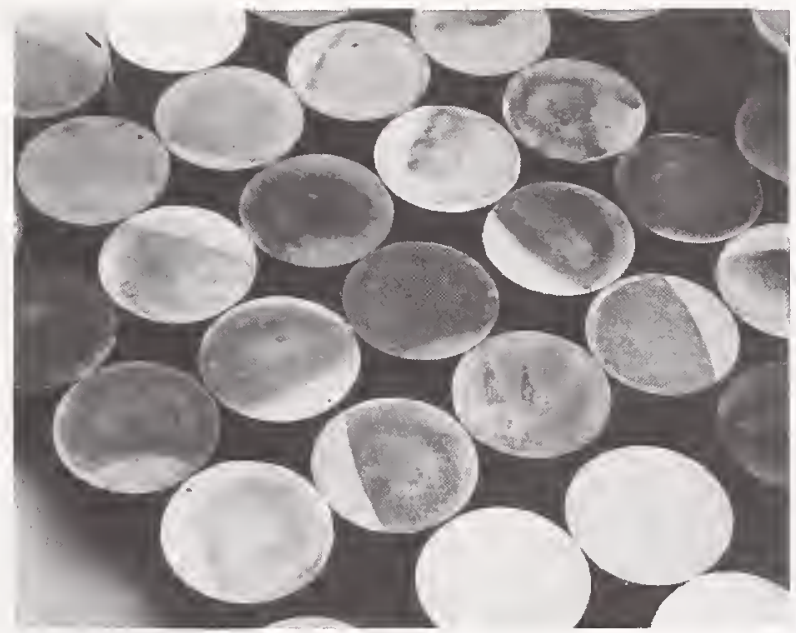

\section{$\Delta$}

After rough castings are separated from the original grid, individual disks of SRM Cl1460 White Cast Iron, are polished for use in optical emission and $x$-ray spectrometric analysis. 


\section{Fission Track Glass}

These SRM's containing uranium at four concentration levels, will aid laboratories, performing fission track analyses, in interlaboratory comparisons of data and in monitoring neutron fluences. The fission track glass standards are certified for neutron flux $\left(\mathrm{n} \cdot \mathrm{cm}^{-2} \cdot \mathrm{sec}^{-1}\right)$ that induced uranium fission in selected wafers. The materials were irradiated in the NBS 10 Megawatt Research Reactor, at two different neutron energies.

Each SRM unit contains four unirradiated glass wafers and two irradiated wafers.

\begin{tabular}{|c|c|c|c|}
\hline SRM & Uranium Content $\mathrm{ng} / \mathrm{g}$ & ${ }^{235} \mathrm{U}$ (Atom Percent) & $\begin{array}{l}\text { NBS Pneumatic Tube Facility/ } \\
\text { Irradiation Time (sec.) }\end{array}$ \\
\hline \multirow[t]{2}{*}{961} & 461.5 & 0.2376 & $\mathrm{RT}-3 / 8$ \\
\hline & . & & $\mathrm{RT}-4 / 12$ \\
\hline $962 a$ & IN PREP & & \\
\hline $963 \mathrm{a}$ & IN PREP & & \\
\hline \multirow[t]{2}{*}{964} & 0.0721 & 0.616 & RT $-3 / 360$ \\
\hline & & & $\mathrm{RT}-4 / 540$ \\
\hline
\end{tabular}

\section{Stable Isotopic}

The isotopic composition of these SRM's has been determined by mass spectrometry, by comparison with mixtures prepared from high-purity separated isotopes. They are intended for use in the assessment of small variations in the isotopic composition of the elements, and for the evaluation of mass discrimination effects encountered in the operation of mass spectrometers.

\begin{tabular}{|c|c|c|c|}
\hline SRM & Isotopic Reference Standards & Element Certified & Wt/Unit (grams) \\
\hline 951 & Boric Acid & Boron & 100 \\
\hline 952 & Boric Acid, $95 \%$ Enriched ${ }^{10} \mathrm{~B}$ & Boron & 0.25 \\
\hline 975 & Sodium Chloride & Chlorine & 0.25 \\
\hline 976 & Copper Metal & Copper & 0.25 \\
\hline 977 & Sodium Bromide & Bromine & 0.25 \\
\hline$\overline{978}$ & Silver Nitrate & Silver & 0.25 \\
\hline 979 & Chromium Nitrate & Chromium & 0.25 \\
\hline 980 & Magnesium Metal & Magnesium & 0.25 \\
\hline *981 & Lead Metal, Natural & Lead & 1.0 \\
\hline *982 & Lead Metal, Equal Atom (206/208) & Lead & 1.0 \\
\hline *983 & Lead Metal, Radiogenic (92\%-206) & Lead & 1.0 \\
\hline 985 & Potassium Chloride, assay and isotopic & Potassium & 1.0 \\
\hline 987 & Strontium Carbonate, assay and isotopic & Strontium & 1.0 \\
\hline 989 & Rhenium, assay and isotopic & Rhenium & pkg. (50) \\
\hline \multirow[t]{2}{*}{990} & Silicon, assay and isotopic & Silicon & wafer, $3 \mathrm{~cm} \mathrm{D}$ \\
\hline & & & $\times 0.2 \mathrm{~cm}$ \\
\hline 991 & Lead-206 Spike, assay and isotopic & Lead & 15 \\
\hline
\end{tabular}

*Sold as a set containing SRM 981, 982, and 983. 


\section{Special Nuclear Containers}

\begin{tabular}{|lll|}
\hline SRM & \multicolumn{1}{c|}{ Type } & Size \\
\hline 9940 & Special Nuclear Container, DOT 6M & 13 gallon \\
\hline 9941 & Special Nuclear Container & 55 gallon \\
\hline 9942 & Special Nuclear Container, Type A & 10 gallon \\
\hline 9943 & Special Nuclear Container, Type A & 55 gallon \\
\hline
\end{tabular}

NOTE: These special packaging materials may be necessary to fill your order, depending upon Department of Energy, Department of Transportation, and Nuclear Regulatory Commission shipping requirements.

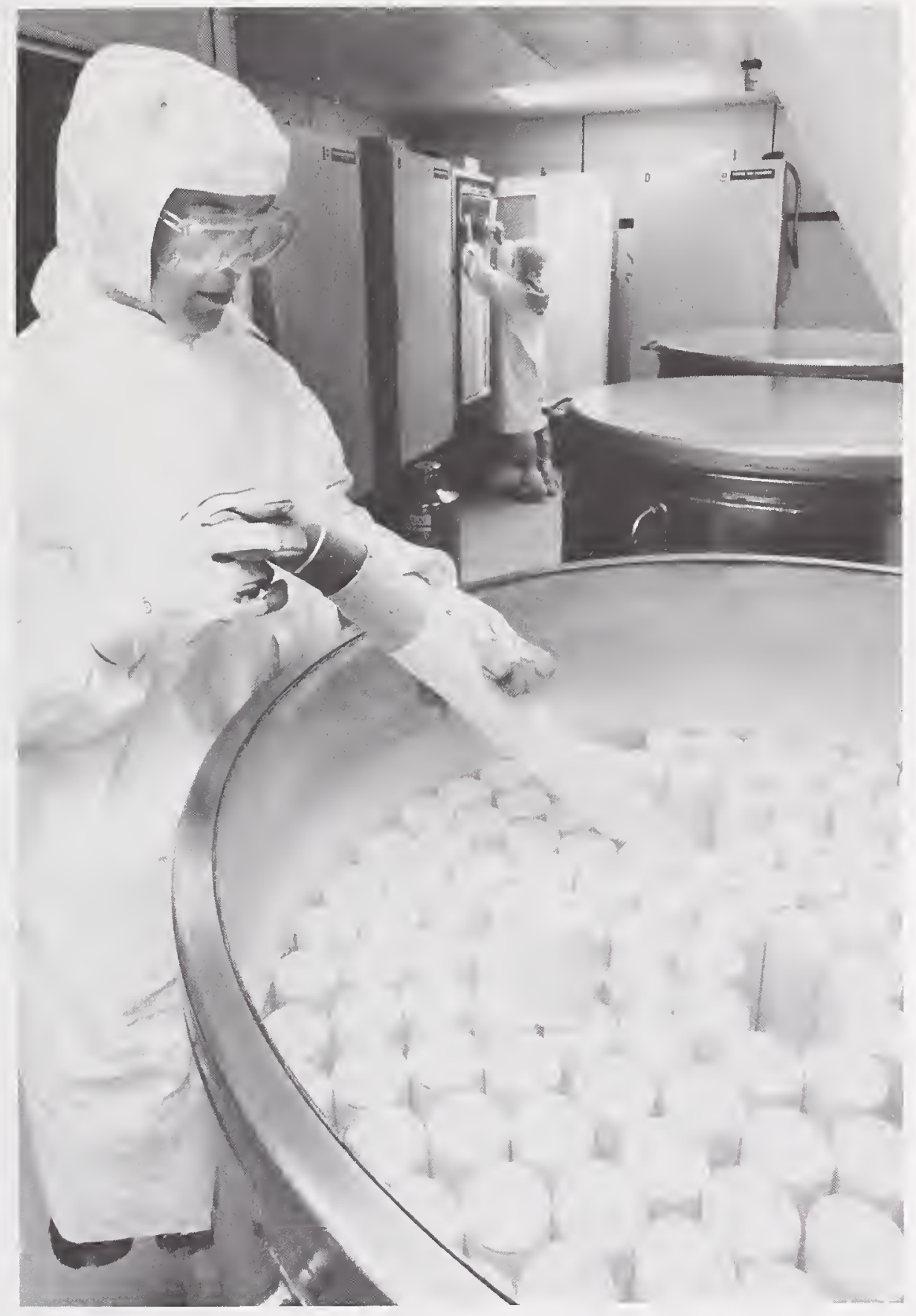

Sally Harrison stores biological samples in a liquid nitrogen freezer as part of the join NBS/EPA Pilot Environmiental Specimien Bank program. 


\section{Certified Physical Properties Standards}

\section{Ion Activity}

These SRM's are intended for use in the preparation of solutions for the calibration of specification electrodes. This includes the $\mathrm{pH}$ and $\mathrm{pD}$ measuring systems.

\section{pH}

These SRM's are furnished as crystals for the preparation of solutions of known hydrogen ion concentration for calibrating and checking the performance of commercially available $\mathrm{pH}$ materials and instruments. They are furnished with certificates giving directions for preparation of the solutions and tables of $\mathrm{pH}$ values at various temperatures.

SRM's 186Ic and 186IIc, 191 and 192, and 922 and 923, are certified for use in admixture only. At an equimolar (0.025 molal) mixture of SRM's $186 \mathrm{Ic}$ and $186 \mathrm{IIc}$, a pH(S) of 6.863 at $25^{\circ} \mathrm{C}$ is obtained. Directions also are furnished for the preparation of a physiological reference solution from 186Ic and 186IIc having a $\mathrm{pH}(\mathrm{S})$ of 7.415 at $25^{\circ} \mathrm{C}$.

\begin{tabular}{|c|c|c|c|}
\hline SRM & Type & $\begin{array}{c}\mathrm{pH}(\mathrm{S}) \\
\left.\text { (at } 25^{\circ} \mathrm{C}\right)\end{array}$ & $\begin{array}{l}\text { Wt/Unit } \\
\text { (grams) }\end{array}$ \\
\hline $185 \mathrm{e}$ & Potassium hydrogen phthalate & 4.004 & 60 \\
\hline $186 \mathrm{Ic}$ & Potassium dihydrogen phosphate & 6.863 & 30 \\
\hline $186 \mathrm{IIC}$ & Disodium hydrogen phosphate & 7.415 & 30 \\
\hline $187 \mathrm{~b}$ & Sodium Tetraborate Decahydrate (Borax) & 9.183 & 30 \\
\hline 188 & Potassium hydrogen tartrate & 3.557 & 60 \\
\hline 189 & Potassium tetroxalate & 1.679 & 65 \\
\hline $191 \mathrm{a}$ & Sodium bicarbonate & 10.011 & 25 \\
\hline $192 \mathrm{a}$ & Sodium carbonate & & 30 \\
\hline 922 & Tris(hydroxymethyl)aminomethane & 7.699 & 25 \\
\hline 923 & Tris(hydroxymethyl)aminomethane hydrochloride & & 35 \\
\hline
\end{tabular}


These SRM's are furnished as crystals for preparation of solutions of known deuterium-ion concentration for the calibration and correction of $\mathrm{pH}$ indicating equipment to indicate $\mathrm{pD}$ data. SRM's $2186 \mathrm{I}$ and 2186II, and 2191 and 2192, are certified for use in admixtures only.

\begin{tabular}{|c|c|c|c|}
\hline SRM & Туре & $\begin{array}{l}\mathrm{pD}(\mathrm{S}) \text { Values } \\
\quad\left(\text { at } 25^{\circ} \mathrm{C}\right)\end{array}$ & $\begin{array}{l}\text { Wt/Unit } \\
\text { (grams) }\end{array}$ \\
\hline 2186 I & Potassium dihydrogen phosphate & 7.428 & 30 \\
\hline $2186 \mathrm{II}$ & Disodium hydrogen phosphate & & 30 \\
\hline 2191 & Sodium bicarbonate & 10.736 & 30 \\
\hline 2192 & Sodium carbonate & & 30 \\
\hline
\end{tabular}

\section{Ion-Selective Electrodes}

These SRM's are certified for the calibration of ion-selective electrodes and have conventional ionic activities based on the Stokes-Robinson hydration theory for ionic strengths greater than 0.1 mole per liter.

\begin{tabular}{|llll|}
\hline & Type & Certified Property & $\begin{array}{c}\text { Wt/Unit } \\
\text { (grams) }\end{array}$ \\
\hline SRM & Sodium Chloride & $\mathrm{pNa}, \mathrm{pCl}$ & 125 \\
\hline 2201 & Potassium Chloride & $\mathrm{pK}, \mathrm{pCl}$ & 160 \\
\hline 2203 & Potassium Fluoride & $\mathrm{pF}$ & 125 \\
\hline
\end{tabular}

\section{Scanning Electron Microscope (SEM)}

This SRM is for use in calibrating the magnification scale and for evaluating the performance of an SEM. SRM 484c has spacings of $1,2,3,5$ and $50 \mathrm{~mm}$ and can be used to calibrate the magnification scale of an SEM from 1000 to $20,000 \mathrm{X}$ to an accuracy of 5 percent or better. This standard is a metallographic crosssection of alternate layers of electro-deposited gold and nickel, encapsulated in copper-filled epoxy, and mounted within a section of stainless steel tubing. If the surface of this SRM were etched by de-sputtering, it would be useful for calibrating optical microscopes.

SRM 2069 consists of graphitized natural fibers that have smooth and uniform edges. Two bundles of the fibers are mounted on an SEM specimen mount for easy use. (Additional fibers are included for use with different mounts.) These fibers have high contrast against the clear background in the center of the mount. A scan of the SEM electron beam across an edge of a fiber will give a CRT trace that will provide a quantitative indication of the performance.

\begin{tabular}{|c|c|c|}
\hline SRM & Type & Size \\
\hline $484 \mathrm{c}$ & SEM Magnification Standard & $11 \mathrm{~mm} \mathrm{D}, 6.5 \mathrm{~mm}$ high \\
\hline 2069 & SEM Performance Standard & $12 \mathrm{~mm} \mathrm{D}, 3 \mathrm{~mm}$ peg \\
\hline
\end{tabular}


These SRM's are for use in calibrating optical microscopes used to measure the widths of opaque lines and clear spaces on integrated-circuit photomasks. They can also be used to calibrate line spacings and line-tospace ratios. The accuracy of a measured linewidth or line spacing is $\pm 0.05 \mathrm{~mm}$ or better. Recommended procedures are provided with each SRM for the adjustment and calibration of measurement systems using transmitted illumination, including filar, image shearing, and video micrometer. They are not recommended for use with partially transmitting materials or in reflected light with opaque materials and it should not be used in a scanning electron microscope. SRM's 474 and 475 are made with anti-reflective chromium on a borosilicate glass substrate. SRM 476 is made with bright chromium.

\begin{tabular}{|llll|}
\hline SRM & \multicolumn{1}{|c|}{ Type } & Spacings & Size \\
\hline 474 & Linewidth Measurement Standard & 0.5 to $12 \mu \mathrm{m}$ & $6.35 \times 6.35 \times 0.15 \mathrm{~cm}$ \\
\hline 475 & Linewidth Measurement Standard & 0.5 to $12 \mu \mathrm{m}$ & $6.35 \times 6.35 \times 0.15 \mathrm{~cm}$ \\
\hline 476 & Linewidth Measurement Standard & 0.5 to $12 \mu \mathrm{m}$ & $6.35 \times 6.35 \times 0.15 \mathrm{~cm}$ \\
\hline
\end{tabular}

The magnetic type thickness gages have a specimen size of $30 \times 30 \mathrm{~mm}$ and are for calibrating coating thickness gages of the magnetic type for the measurement of thickness of nonmagnetic coatings on steel, nickel on steel, or nickel on nonmagnetic substrates. The steel substrates have the magnetic properties of AISI 1010 steel and the nickel coatings have the magnetic properties of an annealed Watts nickel electrodeposit free of cobalt and iron.

These SRM's are often used to measure the thickness of paint and other organic coatings on steel, as well as zinc (galvanized) and other nonmagnetic metallic coatings. The number of different thicknesses required for these calibrations depends on the type of gage and the coating thicknesses to be measured.

The magnetic type thickness gages can be used to estimate magnetic properties of austenitic stainless steel weld metal. Because the magnetic properties of the weld metal are closely related to the ferrite content of the weld, these instruments are used to estimate the ferrite content. The ferrite contents having magnetic properties similar to those of the various coating thickness SRM's have been established by other laboratories.

The gold coating standards were measured by beta-ray backscatter and x-ray fluorescence techniques relative to NBS gold coating materials for which the average weights per unit area were determined by weight and area measurements. They are suitable for the direct calibration of equipment used to measure weight per unit area of gold coating of equivalent purity. From the density and weight per unit area, the instruments can be calibrated in terms of the thickness of the standard. These SRM's have a specimen size of $15 \times 15 \mathrm{~mm}$.

\section{Nonmagnetic Coating on Magnetic Substrate (Copper and Chromium on Steel)}

\begin{tabular}{|c|c|c|c|}
\hline \multirow[b]{2}{*}{ SRM } & \multirow[b]{2}{*}{ Unit Size } & \multicolumn{2}{|c|}{ Nominal Coating Thickness } \\
\hline & & micrometer & milliinch (mil) \\
\hline \multirow[t]{4}{*}{$1361 \mathrm{~b}$} & Set of 4 & 2.5 & 0.1 \\
\hline & & 12 & 0.5 \\
\hline & & 25 & 1.0 \\
\hline & & 50 & 2.0 \\
\hline \multirow[t]{4}{*}{$1362 \mathrm{a}$} & Set of 4 & 40 & 1.6 \\
\hline & & 80 & 3.1 \\
\hline & & 140 & 5.5 \\
\hline & & 200 & 7.9 \\
\hline
\end{tabular}


Nonmagnetic Coating on Magnetic Substrate (Copper and Chromium on Steel) (Continued)

\begin{tabular}{|c|c|c|c|}
\hline \multirow[b]{2}{*}{ SRM } & \multirow[b]{2}{*}{ Unit Size } & \multicolumn{2}{|c|}{ Nominal Coating Thickness } \\
\hline & & micrometer & milliinch (mil) \\
\hline \multirow[t]{4}{*}{$1363 a$} & Set of 4 & 250 & 9.8 \\
\hline & & 400 & 16 \\
\hline & & 500 & 20 \\
\hline & & 650 & 26 \\
\hline \multirow[t]{4}{*}{$1364 a$} & Set of 4 & 820 & 32 \\
\hline & $=$ & 1000 & 39 \\
\hline & & 1500 & 59 \\
\hline & & 2000 & 79 \\
\hline \multirow[t]{4}{*}{1359} & Set of 4 & 50 & 2 \\
\hline & & 140 & 5.5 \\
\hline & & 500 & 20 \\
\hline & & 820 & 32 \\
\hline \multirow[t]{4}{*}{1360} & Set of 4 & 2.5 & 0.1 \\
\hline & & 6 & 0.2 \\
\hline & & 12 & 0.5 \\
\hline & & 20 & 0.8 \\
\hline
\end{tabular}

\section{Magnetic Coating on Magnetic Substrate (Nickel on Steel)}

\begin{tabular}{|lcccc|}
\hline SRM & Unit Size & \multicolumn{2}{c|}{ Nominal Coating Thickness } \\
\cline { 3 - 5 } & micrometer & milliinch (mil) \\
\hline $1365 \mathrm{a}$ & Set of 4 & 3 & 0.1 \\
\hline & & 9 & 0.4 \\
\hline & & 15 & 0.6 \\
\hline & & 20 & 0.8 \\
\hline $1366 \mathrm{a}$ & Set of 4 & 25 & 1.0 \\
\hline & & 35 & 1.4 \\
\hline & & 40 & 1.6 \\
\hline & 50 & 2.0 \\
\hline
\end{tabular}




\section{Magnetic Coating on Non-Magnetic Substrate (Nickel and Chromium on Brass)}

\begin{tabular}{|lcccc|}
\hline & & \multicolumn{2}{c|}{ Nominal Coating Thickness } \\
\cline { 3 - 4 } SRM & Unit Size & micrometer & milliinch (mil) \\
\hline $1367 \mathrm{a}$ & Set of 4 & 3 & 0.1 \\
\hline & & 9 & 0.4 \\
\hline & & 25 & 0.6 \\
\hline
\end{tabular}

\section{Gold Coating on Glass Sealing Alloy-ASTM Designation F15; Fe-53, $\mathrm{Ni}-29$, and $\mathrm{Co}-17$}

\begin{tabular}{|ccccc|}
\hline SRM & Unit Size & $\begin{array}{c}\text { Nominal Coating } \\
\text { Weight } \\
\left(\mathbf{m g} / \mathrm{cm}^{2}\right)\end{array}$ & \multicolumn{2}{c|}{ Nominal Coating Thickness } \\
\hline $1398 \mathrm{a}$ & Set of 4 & 1.5 & micrometer & microinch \\
\hline & & 3.0 & 0.8 & 30 \\
\hline & 6.0 & 1.5 & 60 \\
\hline & 14.0 & 7 & 120 \\
\hline
\end{tabular}

\section{Gold Coating on Nickel}

\begin{tabular}{|c|c|c|c|c|}
\hline \multirow[b]{2}{*}{ SRM } & \multirow[b]{2}{*}{ Unit Size } & \multirow{2}{*}{$\begin{array}{c}\text { Nominal Coating } \\
\text { Weight } \\
\left(\mathrm{mg} / \mathrm{cm}^{2}\right)\end{array}$} & \multicolumn{2}{|c|}{ Nominal Coating Thickness } \\
\hline & & & micrometer & microinch \\
\hline 1379 & Set of 1 & 0.35 & 0.175 & 7 \\
\hline 1380 & Set of 1 & 0.55 & 0.275 & 11 \\
\hline \multirow[t]{4}{*}{$1399 \mathrm{~b}$} & Set of 4 & 1.5 & 0.8 & 30 \\
\hline & & 3.0 & 1.5 & 60 \\
\hline & & 6.0 & 3 & 120 \\
\hline & & 14.0 & 7 & 280 \\
\hline
\end{tabular}

\section{Glass}

\section{Chemical Resistance (Durability) of Glass}

These SRM's are certified for use in checking test methods and for calibrating equipment used to determine the resistance of glass containers to chemical attack. The values given in the table represent the volume of fiftieth-normal sulfuric acid used to titrate to the methyl-red end point the alkaline extract from a crushed sample of glass after exposure to high-purity water at $121^{\circ} \mathrm{C}$.

\begin{tabular}{|c|c|c|c|}
\hline SRM & Type & Unit of Issue & $\mathrm{mL}$ of $\mathrm{N} / 50 \mathrm{H}_{2} \mathrm{SO}_{4}$ \\
\hline 622 & Soda-lime-silica & $2.2 \mathrm{~kg}$ & 7.67 \\
\hline$\overline{623}$ & Borosilicate & $2.2 \mathrm{~kg}$ & 0.34 \\
\hline
\end{tabular}




\section{Electrical Properties of Glass}

SRM 624 is certified over the range of 250 to $350{ }^{\circ} \mathrm{C}$ for use in checking test methods and for calibrating equipment used to determine the dc volume resistivity of glass in accordance with ASTM C657. SRM 774 is certified over the range 100 to $10,000 \mathrm{~Hz}$ for use in checking methods for the determination of dielectric constant and ac loss characteristics of insulating materials in accordance with ASTM D150.

\begin{tabular}{|llcc|}
\hline SRM & Type & Unit of Issue & Approximate Value \\
\hline 624 & Lead-silica, for dc resistivity & $200 \mathrm{~kg}$ & $\log _{10} \rho \sim 9.9 \Omega \cdot \mathrm{cm}$ \\
\hline 774 & $\begin{array}{l}\text { Lead-silica, for dielectric } \\
\text { constant, } 5 \times 5 \times 2.5 \mathrm{~cm}\end{array}$ & block & $\mathrm{K} \sim 7.47$ \\
\hline
\end{tabular}

\section{Glass Viscosity}

SRM's 710, 711, and 717 are furnished as rectangular-shaped bars, and are certified for viscosity between values of $10^{2}$ and $10^{12}$ poises. They are for use in checking the performance of high-temperature viscosity equipment (rotating cylinders) and low-temperature viscosity equipment (fiber elongation, beam-bending, parallel-plates, etc.)

\begin{tabular}{|c|c|c|c|c|c|c|c|c|c|c|c|}
\hline \multirow[b]{2}{*}{ SRM } & \multicolumn{11}{|c|}{ Temperature $\left({ }^{\circ} \mathrm{C}\right)$ at Viscosity (poises) } \\
\hline & $10^{2}$ & $10^{3}$ & $10^{4}$ & $10^{5}$ & $10^{6}$ & $10^{7}$ & $10^{8}$ & $10^{9}$ & $10^{10}$ & $10^{11}$ & $10^{12}$ \\
\hline 710 & 1434.3 & 1181.7 & 1019.0 & 905.3 & 821.5 & 757.1 & 706.1 & 664.7 & 630.4 & 601.5 & 576.9 \\
\hline 711 & 1327.1 & 1072.8 & 909.0 & 794.7 & 710.4 & 645.6 & 594.3 & 552.7 & 518.2 & 489.2 & 464.5 \\
\hline 717 & 1545.1 & 1248.8 & 1059.4 & 927.9 & 831.2 & 757.1 & 698.6 & 651.1 & 611.9 & 579.0 & 550.9 \\
\hline
\end{tabular}

\section{Glass Viscosity Fixpoints}

\begin{tabular}{|c|c|c|c|c|c|}
\hline SRM & Type of Glass & $\begin{array}{l}\text { Unit of } \\
\text { Issue }\end{array}$ & $\begin{array}{l}\text { Softening } \\
\text { Point }{ }^{\circ} \mathrm{C}\end{array}$ & $\begin{array}{l}\text { Annealing } \\
\text { Point }{ }^{\circ} \mathrm{C}\end{array}$ & $\begin{array}{l}\text { Strain } \\
\text { Point }{ }^{\circ} \mathrm{C}\end{array}$ \\
\hline 709 & Extra Dense Lead & $500 \mathrm{~g}$ & 384 & 328 & 311 \\
\hline 710 & Soda Lime-Silica, type 523/586 & $900 \mathrm{~g}$ & 724 & 546 & 504 \\
\hline 711 & Lead-Silica, type $617 / 366$ & $1.3 \mathrm{~kg}$ & 602 & 432 & 392 \\
\hline 712 & Mixed Alkali Lead Silicate $1 / 4$ in patties (6 pcs.) & $225 \mathrm{~g}$ & 528 & 386 & 352 \\
\hline 713 & $\begin{array}{l}\text { Dense Barium Crown } 620 / 60313 / 8 \text { in } \\
\text { diam } \times 5 / 8 \text { in thick gobs (4 pcs.) }\end{array}$ & $225 \mathrm{~g}$ & 738 & 631 & 599 \\
\hline 714 & $\begin{array}{l}\text { Alkaline Earth Alumina Silicate } \\
1 / 4 \text { in diam cane ( } 16 \text { pcs. }-6 \text { in long) }\end{array}$ & $225 \mathrm{~g}$ & 908 & 710 & 662 \\
\hline 715 & $\begin{array}{l}\text { Alkali-Free Aluminosilicate } \\
1 / 4 \text { in diam cane ( } 13 \text { pcs. }-6 \text { in long) }\end{array}$ & $200 \mathrm{~g}$ & 961 & 764 & 714 \\
\hline 716 & Neutral, $1 / 2$ in diam cane ( 6 pcs. -6 in long) & $250 \mathrm{~g}$ & 794 & 574 & 530 \\
\hline 717 & Borosilicate, $4.2 \mathrm{~cm} \times 4.2 \mathrm{~cm} \times 12.5 \mathrm{~cm}$ bar & $450 \mathrm{~g}$ & 720 & 516 & 471 \\
\hline
\end{tabular}




\section{Relative Stress Optical Coefficient}

Three glasses have been certified for relative stress optical coefficient. These glasses will be used to check calibrations of instruments to measure this property, especially by the methods of test proposed by ASTM C770. The glasses are in rectangular-shaped bars.

\begin{tabular}{|lllll|}
\hline SRM & Type of Glass & Unit of Issue & Relative Stress Optical Coefficient at $\lambda=\mathbf{5 4 6 . 1} \mathrm{nm}$ \\
\hline 708 & Lead-Silica, A & $625 \mathrm{~g}$ & Glass A & $\mathrm{C}=2.857 \mathrm{Brewsters}, 10^{-12} \mathrm{~m}^{2} / \mathrm{N}$ \\
\hline & Borosilicate, B & $275 \mathrm{~g}$ & Glass B & $\mathrm{C}=3.652 \mathrm{Brewsters}, 10^{-12} \mathrm{~m}^{2} / \mathrm{N}$ \\
\hline 709 & Extra dense Lead & $500 \mathrm{~g}$ & $\mathrm{C}=-1.359 \mathrm{Brewsters}, 10^{-12} \mathrm{~m}^{2} / \mathrm{N}$ \\
\hline
\end{tabular}

\section{Glass Liquidus Temperature}

This SRM is certified for use in checking test methods and for calibrating equipment used to determine the liquidus temperature of glass by the gradient furnace methods in accordance with ASTM C829.

\begin{tabular}{|cccc|}
\hline SRM & Type & Unit of Issue & Temperature, ${ }^{\circ} \mathrm{C}$ \\
\hline 773 & $\begin{array}{c}\text { Soda-lime-silica, for liquidus temperature } \\
2.5 \times 2.5 \times 0.6 \mathrm{~cm}\end{array}$ & $60 \mathrm{~g}$ & 990 \\
\hline
\end{tabular}

\section{Elastileity}

This SRM is polycrystalline alumina prepared from a single block of material by isostatically cold pressing and then sintering alumina powder containing 0.1 percent magnesium oxide. It is intended for the calibration of apparatus used in the measurement of resonance frequencies from which elastic moduli are calculated. Each bar has been individually measured and calibrated, and all surfaces were machined flat and parallel.

\begin{tabular}{|lcr|}
\hline SRM & Type & Size \\
\hline 718 & Polycrystalline Alumina & $12.7 \times 1.27 \times 0.32 \mathrm{~cm}$ \\
\hline
\end{tabular}

\section{Microhardness}

These SRM's are for use in calibrating and checking the performance of microhardness testers. These test blocks were made by electroforming the test metal on a steel substrate. The hardness numbers are certified at loads of 25, 50, and 100 gram-force for both Vickers and Knoop indenters.

\begin{tabular}{|llll|}
\hline SRM & Type & Hardness & Size \\
\hline 1894 & Bright Copper & $125 \mathrm{KHN}$ & $12.5 \mathrm{~mm}$ square \\
\hline 1895 & Bright Nickel & $550 \mathrm{KHN}$ & $12.5 \mathrm{~mm} \mathrm{square}$ \\
\hline
\end{tabular}




\section{Density}

SRM 217c is certified for density (air saturated at $1 \mathrm{~atm}$ ) at 20,25 , and $30^{\circ} \mathrm{C}$, and may be used to calibrate pycnometers and density balances.

SRM's 1840 and 1841 are certified for density at $20^{\circ} \mathrm{C}$ and may be used to determine the density of solids and liquids by means of hydrostatic weighing.

\begin{tabular}{|lllc|}
\hline \multicolumn{1}{|c|}{ Type } & Density $\mathbf{2 0}{ }^{\circ} \mathbf{C ~} \mathbf{g} / \mathbf{c m}^{\mathbf{3}}$ & Amount \\
\hline $217 \mathrm{c}$ & $2,2,4$ Trimethylpentane & 0.692 & $25 \mathrm{~mL}$ \\
\hline 1840 & Silicon & 2.329 & $100 \mathrm{~g}$ \\
\hline 1841 & Silicon & 2.329 & $200 \mathrm{~g}$ \\
\hline 1825 & Borosilicate Glass & 2.3 & IN PREP \\
\hline 1826 & Soda-Lime Glass & 2.5 & IN PREP \\
\hline 1827 & Lead-Silica Glass & 3.2 & IN PREP \\
\hline
\end{tabular}

\section{Molecular Weight \\ Polymer}

These materials are certified for the properties indicated in the table, such as weight and number average molecular weight, molecular weight distribution, limiting viscosity numbers (intrinsic viscosities) in several solvents, density, and melt flow.

These SRM's have wide application not only in the calibration of instruments used in polymer characterization, such as light scattering photometers, osmometers, gel permeation chromatographs, but also wherever a well-characterized polymer material is needed, as for example in studies of dilute solution behavior, rheology, and polymer crystal physics.

SRM 1475 is accompanied by a series of papers, reprinted from the Journal of Research of the National Bureau of Standards, which describe how the measurements were obtained.

\begin{tabular}{|c|c|c|}
\hline SRM & Type & $\begin{array}{l}\text { Wt/Unit } \\
\text { (grams) }\end{array}$ \\
\hline 705 & Polystyrene, narrow molecular weight distribution, $\mathrm{M}_{w} \approx 179,300, \mathrm{M}_{w} / \mathrm{M}_{\mathrm{n}} \approx 1.07$ & 5 \\
\hline 706 & Polyst yrene, broad molecular weight distribution, $\mathrm{M}_{\mathrm{w}} \approx 257,800, \mathrm{M}_{\mathrm{w}} / \mathrm{M}_{\mathrm{n}} \approx 2.1$ & 18 \\
\hline 1475 & Polyethylene, linear, $\mathrm{M}_{\mathrm{w}} \approx 52,000, \mathrm{M}_{\mathrm{w}} / \mathrm{M}_{\mathrm{n}} \approx 2.9$ & 50 \\
\hline 1476 & Polyethylene, branched & 50 \\
\hline 1478 & Polystyrene, narrow molecular weight distribution, $M_{w} \approx 37,400, M_{w} / M_{n} \approx 1.04$ & 2 \\
\hline 1479 & Polystyrene, narrow molecular weight distribution, $\mathrm{M}_{\mathrm{w}} \approx 1,050,000$ & 2 \\
\hline$\underline{1482}$ & Polyethylene, linear, $M_{w} \approx 13,600$ & 1 \\
\hline 1483 & Polyethylene, linear, $\mathbf{M}_{w} \approx 32,100$ & 1 \\
\hline 1484 & Polyethylene, linear, $\mathbf{M}_{\mathrm{w}} \approx 119,600$ & 1 \\
\hline
\end{tabular}




\section{Polymer (Continued)}

\begin{tabular}{|c|c|c|c|c|c|c|c|c|c|c|}
\hline Property & Method & 705 & 706 & 1475 & 1476 & 1478 & 1479 & 1482 & 1483 & 1484 \\
\hline \multirow{2}{*}{$\begin{array}{l}\text { Molecular Weight: } \\
\text { Weight Average }\end{array}$} & & & & & & & & & & \\
\hline & (Light Scattering) & $\mathrm{x}$ & $\mathrm{X}$ & $\mathrm{X}$ & & & $\mathrm{X}$ & $\mathrm{X}$ & $\mathrm{X}$ & $\mathrm{X}$ \\
\hline & (Sedimentation & $\mathrm{X}$ & $\mathrm{X}$ & & & $\mathrm{X}$ & & & & \\
\hline & Equilibrium) & & & & & & & & & \\
\hline & (Gel Permeation & & & $\mathrm{X}$ & & & & & & \\
\hline & Chromatography-GPC) & & & & & & & & & \\
\hline Number Average & (Osmometry) & $\mathrm{X}$ & & & & $\mathrm{X}$ & & $\mathrm{X}$ & $\mathrm{X}$ & $\mathrm{X}$ \\
\hline & (GPC) & & & $\mathrm{X}$ & & & & & & \\
\hline Molecular Weight Distribution & $(\mathrm{GPC})$ & & & $\mathrm{X}$ & & & & & & \\
\hline Limiting Viscosity Number & (Capillary Viscometer) & & & & & $\mathrm{X}$ & & & & \\
\hline Toluene $25^{\circ} \mathrm{C}$ & & & & & & & & & & \\
\hline Benzene $25^{\circ} \mathrm{C}$ & & $\mathrm{X}$ & $\mathrm{X}$ & & & & & & & \\
\hline Benzene $35^{\circ} \mathrm{C}$ & & $\mathrm{X}$ & & & & & & & & \\
\hline Cyclohexane $35^{\circ} \mathrm{C}$ & & $\mathrm{X}$ & $\mathrm{X}$ & & & & & & & \\
\hline 1-Chloronaphthalene $130^{\circ} \mathrm{C}$ & & & & $\mathrm{X}$ & $\mathrm{X}$ & & & $X$ & $\mathrm{X}$ & $\mathrm{X}$ \\
\hline 1,2,4-trichlorobenzene $130^{\circ} \mathrm{C}$ & & & & $\mathrm{X}$ & $\mathrm{x}$ & & & $\mathrm{X}$ & $X$ & $\mathrm{X}$ \\
\hline Decahydronaphthalene $130^{\circ} \mathrm{C}$ & & & & $\mathrm{X}$ & $\mathrm{X}$ & & & & & \\
\hline Melt Flow & (ASTM) & & & $\mathrm{X}$ & $\mathrm{X}$ & & & & & \\
\hline Density & (ASTM) & & & $\mathrm{X}$ & $\mathrm{X}$ & & & & & \\
\hline Heat Capacity & (Adiabatic) & $\mathrm{X}$ & & $\mathrm{X}$ & & & & & & \\
\hline
\end{tabular}

\section{Rheology}

This SRM is intended for the calibration and checking of instruments used in polymer technology and science for the determination of rheological properties of polymer melts or solutions. It is certified for Rate of Shear, Viscosity, and First Normal Stress Difference at $25^{\circ} \mathrm{C}$.

\begin{tabular}{|lcr}
\hline SRM & Type & Unit size \\
\hline 1490 & Polyisobutylene Solution in Cetane & $250 \mathrm{~mL}$ \\
\hline
\end{tabular}

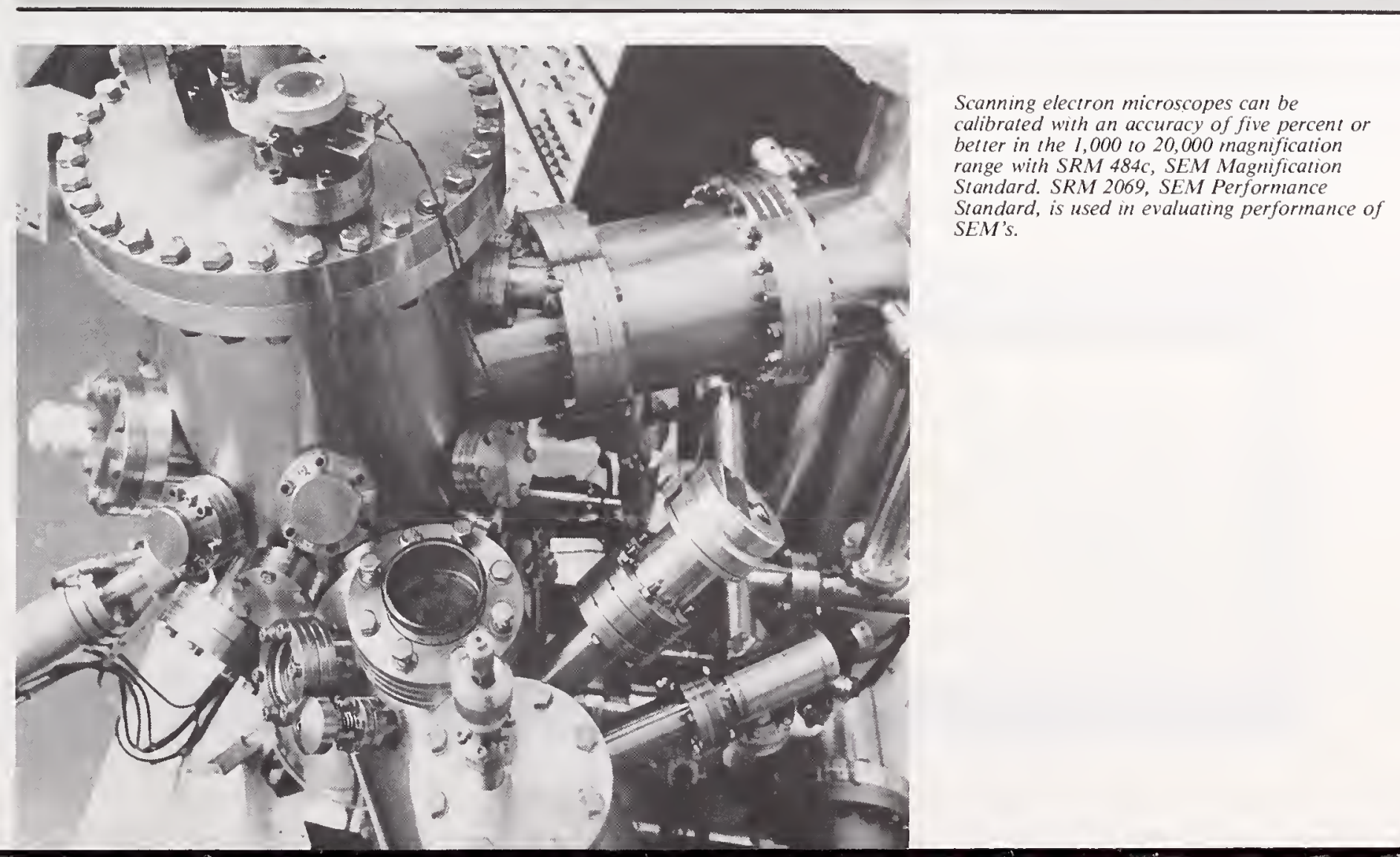




\section{Superconductive Thermometric Fixed Point Devices}

Each device is composed of small cylinders of high purity material mounted in a threaded copper stud and enclosed by a mutual inductance coil set. SRM 767a is intended to provide fixed points on the 1976

Provisional 0.5 to $30 \mathrm{~K}$ Temperature Scale (EPT-76). Both SRM's should prove particularly valuable to users of ${ }^{3} \mathrm{He}-{ }^{4} \mathrm{He}$ dilution refrigerators, in which direct calibrations on the liquid helium vapor pressure-

temperature scales are difficult, and to those who wish to determine the temperature reproducibility of physical phenomena or of cryogenic equipment.

\begin{tabular}{|llll|}
\hline SRM & \multicolumn{1}{|c|}{ Type } & Material & $\begin{array}{c}\text { Nominal } \\
\text { Temperature (K) }\end{array}$ \\
\hline $767 \mathrm{a}$ & Superconductive Thermometric Fixed Point Device & Niobium & 9.3 \\
\hline & & Lead & 7.2 \\
\hline & Indium & 3.4 \\
\hline & Aluminum & 1.2 \\
\hline & & Zinc & 0.9 \\
\hline & Superconductive Thermometric Fixed Point Device (Low) & Cadmium & 0.5 \\
\hline & Gold-Indium & 0.205 \\
\hline & Gold-Aluminum & 0.157 \\
\hline & Iridium & 0.098 \\
\hline & Beryllium & 0.024 \\
\hline
\end{tabular}

\section{Freezing Point}

\section{Defining Fixed Points-International Practical Temperature Scale}

These SRM's are of such purity that they are suitable for defining fixed points for the International Practical Temperature Scale of 1968.

\begin{tabular}{|lllll}
\hline SRM & Type & Temperature ${ }^{\circ} \mathbf{C}$ & $\begin{array}{c}\text { Wt/Unit } \\
\text { (grams) }\end{array}$ \\
\hline 740 & Zinc & 419.58 & 350 \\
\hline 741 & Tin & 231.9681 & 350 \\
\hline
\end{tabular}

\section{Secondary Reference Points}

These SRM's are intended for use in calibration of thermometers, thermocouples, and other temperature measuring devices. The temperatures certified are in accord with the International Practical Temperature Scale of 1968.

\begin{tabular}{|llcc|}
\hline SRM & Type & Temperature ${ }^{\circ} \mathbf{C}$ & $\begin{array}{c}\text { Wt/Unit } \\
\text { (grams) }\end{array}$ \\
\hline $42 \mathrm{~g}$ & Tin & 231.967 & 350 \\
\hline $43 \mathrm{~h}$ & Zinc & $* 419.58$ & 350 \\
\hline $44 \mathrm{f}$ & Aluminum & 660.3 & 200 \\
\hline $45 \mathrm{~d}$ & Copper & 1084.8 & 3 \\
\hline $49 \mathrm{e}$ & Lead & 327.493 & 450 \\
\hline 743 & Mercury & -38.841 & 600 \\
\hline
\end{tabular}

*SRM $43 \mathrm{~h}$ is less pure than SRM 740 and has a freezing point $0.001{ }^{\circ} \mathrm{C}$ lower. 


\section{Melting Point}

\begin{tabular}{|llcc|}
\hline SRM & \multicolumn{1}{|c|}{ Type } & Temperature ${ }^{\circ} \mathrm{C}$ & $\begin{array}{c}\text { Wt/Unit } \\
\text { (grams) }\end{array}$ \\
\hline 742 & Alumina, $99.9+\%$ & 2053 & 10 \\
\hline 1968 & Gallium, $99.9999+\%$ & 29.7723 & 25 \\
\hline 1969 & Rubidium & 38 & IN PREP \\
\hline
\end{tabular}

NBS calibrates liquid in glass, thermocouple, and resistance thermometers. For information, call 301-921-2805.

\section{Calorimetric}

These SRM's are intended to relate the gain or loss of energy and work experienced during a chemical reaction or by change of temperature to the units of energy and work as defined by the National Measurement System. This system uses the units prescribed by the International System of Units (SI). The unit for energy and work under this system is the joule, which is related to the calorie by the equation: 4.184 joule $=1$ calorie.

\section{Combustion Calorimetric}

\begin{tabular}{|llcc}
\hline SRM & \multicolumn{1}{c}{ Type } & Approximate Heat of Combustion & Unit Amount \\
\hline $39 \mathrm{i}$ & Benzoic Acid & $26.4 \mathrm{KJ} / \mathrm{g}$ & $30 \mathrm{~g}$ \\
\hline $217 \mathrm{c}$ & $2,2,4-$ Trimethylpentane & 47.7 & $25 \mathrm{~mL}$ \\
\hline 2152 & Urea & -10.5 & $30 \mathrm{~g}$ \\
\hline
\end{tabular}

\section{Solution Calorimetric}

\begin{tabular}{|ccc}
\hline SRM & Type & $\begin{array}{c}\text { Wt/Unit } \\
\text { (grams) }\end{array}$ \\
\hline $724 \mathrm{a}$ & $\begin{array}{c}\text { Tris(hydroxymethyl) aminomethane } \\
\text { (Hydrochloric Acid and Sodium Hydroxide Solution Calorimetry) }\end{array}$ & 50 \\
\hline 1654 & $\alpha \cdot$ Quartz (Hydrofluoric Acid Solution Calorimetry) & 25 \\
\hline 1655 & Potassium Chloride (Water Solution Calorimetry & 30 \\
\hline
\end{tabular}

\section{Heat Source Calorimetric}

\begin{tabular}{|lll|}
\hline SRM & Type & $\begin{array}{c}\text { Wt/Unit } \\
\text { (grams) }\end{array}$ \\
\hline 1651 & Zirconium-barium chromate heat source powder $(\mathrm{ca} 350 \mathrm{cal} / \mathrm{g})$ & 50 \\
\hline 1652 & Zirconium-barium chromate heat source powder $(\mathrm{ca} 390 \mathrm{cal} / \mathrm{g})$ & 50 \\
\hline 1653 & Zirconium-barium chromate heat source powder $(\mathrm{ca} 425 \mathrm{cal} / \mathrm{g})$ & 50 \\
\hline
\end{tabular}




\section{Calorimetric (Continued) Enthalpy and Heat Capacity}

\begin{tabular}{|c|c|c|c|}
\hline SRM & Type & Temperature Range (K) & Unit Size \\
\hline 705 & Polystyrene, powder & $10-350$ & $5 \mathrm{~g}$ \\
\hline 720 & Sapphire, (synthetic $\mathrm{Al}_{2} \mathrm{O}_{3}$ ) small rods & $0-2250$ & $15 \mathrm{~g}$ \\
\hline 781-D1 & Molybdenum, sintered rod & $273.15-2800$ & $10 \mathrm{~cm} \times 0.32 \mathrm{~cm} \mathrm{D}$ \\
\hline 781-D2 & Molybdenum, sintered rod & $273.15-2800$ & $10 \mathrm{~cm} \times 0.64 \mathrm{~cm} \mathrm{D}$ \\
\hline 782 & Tungsten, rod & $273-1200$ & $10 \mathrm{~cm} \times 0.32 \mathrm{~cm} \mathrm{D}$ \\
\hline 1475 & Polyethylene, powder & $5-360$ & $50 \mathrm{~g}$ \\
\hline
\end{tabular}

\section{Vapor Pressure}

These SRM's are intended for use in the testing and calibration of vapor pressure measurent apparatus and techniques.

\begin{tabular}{|lllcl|}
\hline SRM & Type & $\begin{array}{c}\text { Pressure Range } \\
\text { (atmosphere) }\end{array}$ & Temperature Range (K) & Unit Size \\
\hline 745 & Gold & $10^{-3}$ to $10^{-8}$ & $1300-2100$ & Wire $1.44 \mathrm{~mm} \times 152 \mathrm{~mm}$ \\
\hline 746 & Cadmium & $10^{-4}$ to $10^{-11}$ & $350-594$ & Rod $6.4 \mathrm{~mm} \times 64 \mathrm{~mm}$ \\
\hline 748 & Silver & $10^{-3}$ to $10^{-12}$ & $800-1600$ & Rod $6.4 \mathrm{~mm} \times 64 \mathrm{~mm}$ \\
\hline
\end{tabular}

\section{Thermal Conductivity}

SRM's $1450 \mathrm{~b}$ and 1451 are intended for use in calibrating and verifying guarded hot-plate apparatus (ASTM C177) and heat-flow meters (ASTM C518) used to determine the thermal resistance of thermal insulation materials. SRM's 1460 through 1468 cover the high to low conductivity range of metals and are useful in intercomparing and calibrating thermal conductivity apparatus.

\begin{tabular}{|c|c|c|c|c|}
\hline SRM & Type - & Dimension (mm) & Temperature Range (K) & $\begin{array}{l}\text { Conductivity at } \\
293 \mathrm{~K}(\mathrm{w} / \mathrm{m} \cdot \mathrm{K})\end{array}$ \\
\hline $1450 \mathrm{~b}$ & Fibrous Glass Board & $60 \times 60 \times 2.54$ & $260-330$ & 0.03 \\
\hline 1451 & Fibrous Glass Batt & $60 \times 60 \times 2.54$ & $260-330$ & 0.039 \\
\hline 1460 & Stainless Steel & $6.4 \mathrm{D}, 50$ length & $5-1200$ & 14.1 \\
\hline 1461 & Stainless Steel & $12.7 \mathrm{D}, 50$ length & $5-1200$ & 14.1 \\
\hline 1462 & Stainless Steel & $34 \mathrm{D}, 50$ length & $5-1200$ & 14.1 \\
\hline 1463 & Electrolytic Iron & $6.4 \mathrm{D}, 50$ length & $6-1000$ & 77.9 \\
\hline 1464 & Electrolytic Iron & $31.7 \mathrm{D}, 50$ length & $6-1000$ & 77.9 \\
\hline 1465 & Sintered Tungsten & $3.2 \mathrm{D}, 50$ length & $4-3000$ & 173 \\
\hline 1466 & Sintered Tungsten & $6.4 \mathrm{D}, 50$ length & $4-3000$ & 173 \\
\hline 1467 & Arc-Cast Tungsten & $8.3 \mathrm{D}, 50$ length & $4-3000$ & 173 \\
\hline$\overline{1468}$ & Arc-Cast Tungsten & $10.2 \mathrm{D}, 50$ length & $4-3000$ & 173 \\
\hline
\end{tabular}




\section{Thermal Expansion}

These SRM's cover the temperature range from 20 to $1800 \mathrm{~K}$ having coefficients of thermal expansion over the range of 0.5 to $17 \times 10^{-6} \mathrm{~K}$.

\begin{tabular}{|llccc|}
\hline SRM & Type & Temperature Range (K) & Diameter (mm) & Length (mm) \\
\hline $731-$ L1 & Borosilicate Glass & $80-680$ & 6.4 & 51 \\
\hline $731-\mathrm{L} 2$ & Borosilicate Glass & $80-680$ & 6.4 & 102 \\
\hline $731-\mathrm{L} 3$ & Borosilicate Glass & $80-680$ & 6.4 & 152 \\
\hline $736 \mathrm{a}$ & Copper (IN PREP) & $20-800$ & 6.4 & 51 \\
\hline $737 \quad$ & Tungsten & $80-1800$ & 6.4 & 51 \\
\hline & & $293-750$ & & 51 \\
\hline $738 \quad$ & Stainless Steel & $80-1000$ & 6.4 & 51 \\
\hline $739-\mathrm{L} 1$ & Fused Silica & $80-1000$ & 6.4 & 102 \\
\hline $739-\mathrm{L} 2$ & Fused Silica & $80-1000$ & 6.4 & 152 \\
\hline $739-\mathrm{L} 3$ & Fused Silica & 6.4 & \\
\hline
\end{tabular}

\section{Thermocouple Materials}

These SRM's are intended to serve as a convenient mechanism for the comparison of manufactured wire to standard reference thermocouple tables.

\begin{tabular}{|lll|}
\hline SRM & \multicolumn{1}{c|}{ Type } & \multicolumn{1}{c|}{ Form } \\
\hline 733 & Silver-28 Atomic Percent Gold & Wire: $32 \mathrm{AWG}(0.2019 \mathrm{~mm} \mathrm{D,} \mathrm{3} \mathrm{meters} \mathrm{long}$ \\
\hline 1967 & Platinum, High-Purity $(99.999+\%)$ & Wire: $0.51 \mathrm{~mm} \mathrm{D,1} \mathrm{meter} \mathrm{long}$ \\
\hline
\end{tabular}

\section{Magnetic}

\section{Magnetic Susceptibility}

These SRM's are intended for use in the calibration of instruments used to measure magnetic susceptibility.

\begin{tabular}{|lll|}
\hline SRM & Type & Form/Unit \\
\hline $763-1$ & Aluminum & Cylinder 3 mm diameter $\times 3 \mathrm{~mm}$ \\
\hline $764-1$ & Platinum & Cylinder $3 \mathrm{~mm} \mathrm{diameter} \times 3 \mathrm{~mm}$ \\
\hline $765-1$ & Palladium & Cylinder $3 \mathrm{~mm} \mathrm{diameter} \times 3 \mathrm{~mm}$ \\
\hline $766-1$ & Manganese Fluoride & Cube $3 \times 3 \times 3 \mathrm{~mm}$ \\
\hline
\end{tabular}

\section{Magnetic Moment}

This SRM is intended for the calibration of instruments used to measure magnetic moment.

\begin{tabular}{|llr|}
\hline SRM & Type & Size \\
\hline $772 \quad$ Nickel Sphere & $2.4 \mathrm{~mm} \mathrm{D}$ \\
\hline
\end{tabular}




\section{Paramagnetic Resonance}

This SRM is intended for use in electron paramagnetic resonance (EPR) measurements for determining the number of active paramagnetic centers in a test sample. This SRM consists of two pieces of synthetic ruby.

\begin{tabular}{|lcc|}
\hline SRM & Type & Form \\
\hline 2601 & Crystalline $\mathrm{Al}_{2} \mathrm{O}_{3} ; \mathrm{Cr}^{3+}$ (Ruby). Set of two pieces & $1.5 \times 1.5 \times 0.5 \mathrm{~mm}$ \\
\hline & & $0.5 \times 0.5 \times 4 \mathrm{~mm}$ \\
\hline
\end{tabular}

\section{Opticall}

\section{Spectrophotometric}

\section{Filters for Spectrophotometry and Luminescence}

The spectrophotometric SRM's are intended primarily for use in verifying the accuracy of the transmittance scale of spectrophotometers. The luminescence SRM provides relative emission spectra to determine spectral responsivity and to verify the accuracy of spectrofluorimeters. All of these SRM's provide a means of interlaboratory comparison of data.

\section{Glass Filters: SRM 930D}

This SRM consists of three neutral glass filters. The glass filters have transmittances of approximately 10,20 , and 30 percent. Each filter is individually calibrated and certified for absorbance and transmittance at wavelengths of $440,465,546.1,590$, and $635 \mathrm{~nm}$. The $546.1 \mathrm{~nm}$ wavelength coincides with the mercury emission line. Unit: Set of 3 filters, 4 holders.

\section{Liquid Filters: SRM 931C}

These filters are absorbance standards for use in ultraviolet and visible spectrophotometry. This SRM consists of three sets of four vials, each containing a blank solution and three solutions of different concentrations of an absorbing liquid. Each vial contains approximately $10 \mathrm{~mL}$ of solution. The net absorbances are certified for each concentration at wavelengths 302, 395, 512, and $678 \mathrm{~nm}$. Unit: Set of 12 vials.

\section{Quartz Cuvette: SRM 932}

This SRM is an all-quartz rectangular parallelepiped cuvette designed to fit the holder of conventional spectrophotometers. The distances between the parallel, optically-transparent windows are measured at 10 positions along the vertical axis. The cuvettes range in pathlength between 9.97 and $10.03 \mathrm{~mm}$, and the inner surfaces of the opposite windows are parallel within $\pm 0.002 \mathrm{~mm}$. Each cuvette is certified for pathlength and parallelism of the windows to within $\pm 0.0005 \mathrm{~mm}$. Unit: 1 each.

\section{Potassium Dichromate: SRM 935}

This SRM consists of crystalline potassium dichromate of established purity certified for use as an ultraviolet absorbance standard. Solutions made with this SRM in $0.001 \mathrm{~N}$ perchloric acid are certified for their apparent specific absorbances, $\epsilon_{\mathrm{a}}$, at $23.5^{\circ} \mathrm{C}$ and wavelengths of $235,257,313,345$, and $350 \mathrm{~nm}$. Unit: 15 grams.

\section{Quinine Sulfate Dihydrate: SRM 936}

This SRM consists of powdered quinine sulfate dihydrate of known purity certfied for use as a spectrofluorimetric emission standard. A solution made with this SRM in $0.1 \mathrm{~N}$ perchloric acid is certified for its molecular emission spectrum, $\mathrm{E}(\mathrm{g})$ at $25.0{ }^{\circ} \mathrm{C}$ over the wavelength range of 375.0 to $675 \mathrm{~nm}$. Unit: 1 gram.

Didymium-Oxide Glass Filters: SRM's 2009 and 2010

These wavelength SRM's are for use in checking the wavelength scale of spectrophotometers between 400 and $760 \mathrm{~nm}$ for bandpasses between 1.5 and $10.5 \mathrm{~nm}$. SRM 2009 is approximately $1 \mathrm{~cm}$ wide by $3 \mathrm{~cm}$ high and is supplied in a holder which fits in the place of a standard analytical cuvette. SRM 2010 is in the form of a square approximately $5.1 \mathrm{~cm}$ by $5.1 \mathrm{~cm}$. 


\section{Spectrophotometric (Continued)}

Glass Filter: SRM 2030

This SRM consists of one neutral glass filter. It is intended as a reference source for one-point verification of the transmittance and abso bance scales of spectrophotometers at a wavelength of $465 \mathrm{~nm}$ and a nominal 30 percent transmittance. Unii: 1 filter, 2 holders.

Metal-on-Quartz Filters: SRM 2031

This SRM consists of three filters mounted in metal holders and an empty holder; all holders are equipped with shutters. Two of the filters have an evaporated layer of semitransparent metal sandwiched between two quartz plates that have been assembled by optical contact. The third filter consists of two clear quartz plates assembled by the same technique. Each filter is individually calibrated at 250, 300, 340, 400, 465, 546, 590, and $635 \mathrm{~nm}$. Unit: Set of 3 filters, 4 holders.

\section{Potassium Iodide: SRM 2032}

This SRM consists of crystalline KI of established purity for use as a stray light standard in the ultraviolet. Aqueous solutions made with this material are certified for their specific absorbance at $23.5^{\circ} \mathrm{C}$ over a wavelength range from 240 to $280 \mathrm{~nm}$. Unit: 25 grams.

Potassium Iodide with Attenuator: SRM 2033

SRM 2033 consists of the same material used for SRM 2032 plus a reference beam attenuator for extending the dynamic range of the stray light test.

\begin{tabular}{|c|c|c|}
\hline SRM & Type & Unit \\
\hline $930 \mathrm{D}$ & Glass Filters for Spectrophotometry & Set: 3 filters, 4 holders \\
\hline $931 \mathrm{c}$ & Liquid Filters for Spectrophotometry & Set: 12 vials \\
\hline 932 & Quartz Cuvette for Spectrophotometry & 1 each \\
\hline$\overline{935}$ & $\begin{array}{l}\text { Crystalline Potassium Dichromate for } \\
\text { Use as an Ultraviolet Absorbance Standard }\end{array}$ & 15 grams \\
\hline 936 & Quinine Sulfate Dihydrate & 1 gram \\
\hline 2009 & Didymium-oxide glass & 1 filter in holder \\
\hline 2010 & Didymium-oxide glass & $51 \times 51 \mathrm{~mm}$ \\
\hline 2030 & Glass Filter for Transmittance Measurement & 1 filter, 2 holders \\
\hline 2031 & Metal-on-Quartz Filters for Spectrophotometry & Set: 3 filters, 4 holders \\
\hline 2032 & Potassium Iodide for Use as a Stray Light Standard & 25 grams \\
\hline 2033 & $\begin{array}{l}\text { Potassium Iodide with Attenuator for Use as Stray } \\
\text { Light Standard }\end{array}$ & IN PREP \\
\hline
\end{tabular}

For further information regarding calibration services, call 301-921-2805.

\section{Reflectance}

These SRM's are intended primarily for calibration of the reflectance scale of integrating sphere reflectometers used in evaluating materials for solar energy collectors and for calibration of reflectometers used in evaluating the appearance of polished metals and metal plated objects. 


\section{Specular Spectral Reflectance}

SRM's 2003a and 2008a are first-surface mirrors of vacuum deposited aluminum and gold, respectively on glass that are certified for near-normal reflectance over the wavelength range of 250 to $2500 \mathrm{~nm}$. SRM's 2023 , 2024 and 2025 are second-surface mirrors of vacuum deposited aluminum on a fused quartz plate and covered with a second plate of fused quartz. These mirrors are also certified for near-normal reflectance from 250 to $2500 \mathrm{~nm}$ and for a few wavelengths at 15, 30, 45 and 60 degrees from normal. SRM 2025 has a small angle between the front and rear surfaces.

\begin{tabular}{|lrr|}
\hline SRM & \multicolumn{1}{c}{ Type } & Size \\
\hline $2003 \mathrm{a}$ & First Surface, Aluminum on Glass & $5.1 \mathrm{~cm}$ D \\
\hline $2008 \mathrm{a}$ & First Surface, Gold on Glass & $5.1 \mathrm{~cm} \mathrm{D}$ \\
\hline 2023 & Second Surface, Aluminum on Fused Quartz & $5.1 \times 5.1 \mathrm{~cm}$ \\
\hline 2024 & Second Surface, Aluminum on Fused Quartz & $2.5 \times 10.2 \mathrm{~cm}$ \\
\hline 2025 & Second Surface, Aluminum on Fused Quartz with wedge & $2.5 \times 10.2 \mathrm{~cm}$ \\
\hline
\end{tabular}

For further information regarding calibration services, call 301-921-2805.

\section{Directional-Hemispherical Reflectance}

SRM's 2015 and 2016 are made from opal glass and are certified for near-normal reflectance from 400 to 750 $\mathrm{nm}$. SRM's 2019a and 2020 are white ceramic tiles certified from 350 to $2500 \mathrm{~nm}$. SRM's 2021 and 2022 are black porcelain enamel squares certified from 280 to $2500 \mathrm{~nm}$. These last four SRM's are primarily certified for near-normal reflectance but are also certified for reflectance at a few wavelengths at $15,30,45$ and 60 degrees from normal.

\begin{tabular}{|llc|}
\hline SRM & \multicolumn{1}{c|}{ Type } & Size \\
\hline 2015 & Opal Glass & $2.5 \times 5.0 \times 0.64 \mathrm{~cm}$ \\
\hline 2016 & Opal Glass & $10 \times 10 \times 0.64 \mathrm{~cm}$ \\
\hline $2019 \mathrm{~b}$ & White Ceramic Tile & $5.1 \times 5.1 \times 0.81 \mathrm{~cm}$ \\
\hline 2020 & White Ceramic Tile & $3.8 \times 7.6 \times 0.81 \mathrm{~cm}$ \\
\hline 2021 & Black Porcelain Enamel & $5.1 \times 5.1 \times 0.20 \mathrm{~cm}$ \\
\hline 2022 & Black Porcelain Enamel & $2.5 \times 2.5 \times 0.20 \mathrm{~cm}$ \\
\hline
\end{tabular}

For further information regarding calibration services, call 301-921-2805.

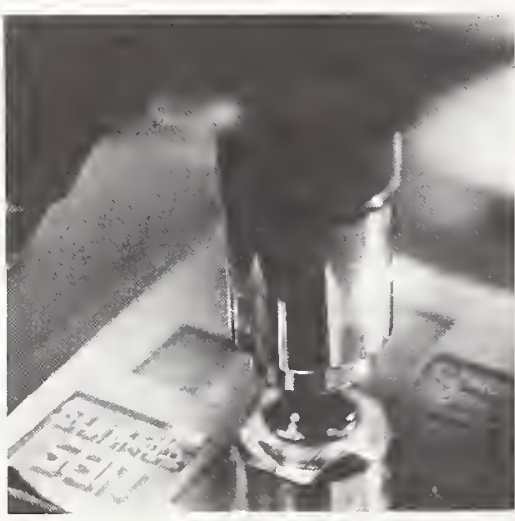

Samuel Jones examines SRM 475, Optical Microscope Linewidth Measurement Standard, used in calibrating optical microscopes that measure linewidths from 0.5 to $10 \mu \mathrm{m}$.

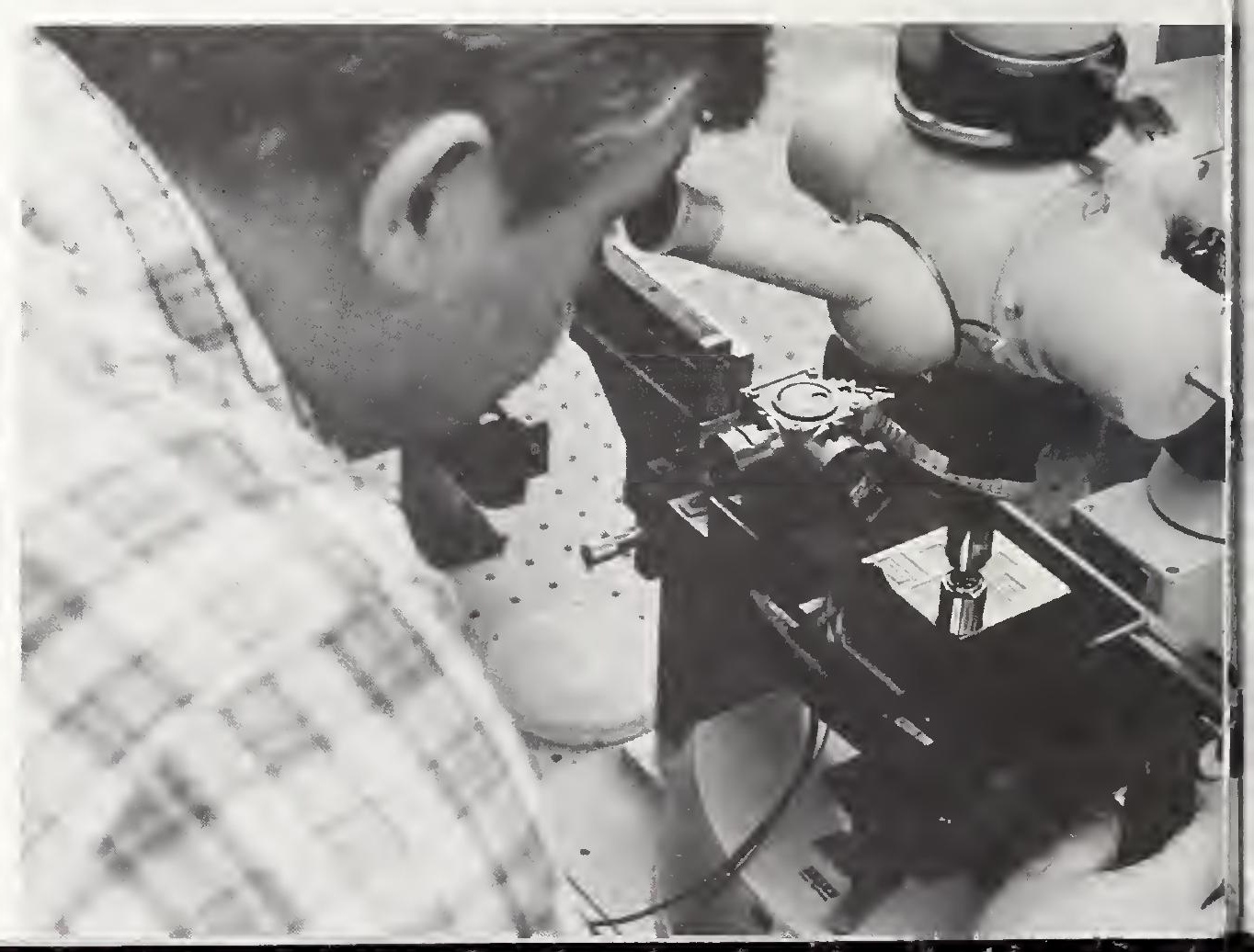




\section{Refractive Index}

SRM's $211 \mathrm{c}$ and $217 \mathrm{c}$ are certified for refractive index at 20,25 and $30^{\circ} \mathrm{C}$, from 435.8 to $667.8 \mathrm{~nm}$ for seven wavelengths, and are available in 5 and $25 \mathrm{~mL}$ ampoules.

SRM's 1820 and 1822 are certified for refractive index at thirteen wavelengths from $404.7 \mathrm{~nm}$ to 706.5 $\mathrm{nm}$. These SRM's are designed for calibrating refractometers and certifying refractive index immersion liquids, and should provide a basis for accurate measurements of refractive index and dispersion. They consist of two rectangular glass slabs: one slab has polished faces and is to be used to check the performance of a refractometer; the second slab is unpolished and can be broken into fragments to certify the refractive index of immersion liquids by microscope methods.

SRM 1823 consists of two silicone liquids that are chemically and thermally stable. The liquids are miscible and span the refractive index range of a variety of glasses and glass fibers that are examined microscopically by immersion techniques. Used independently, the liquids are suitable for the calibration of refractometers. These liquids are certified for refractive index at ten wavelengths from 435.8 to $667.8 \mathrm{~nm}$, at temperatures of $20,40,60$, and $80^{\circ} \mathrm{C}$.

\begin{tabular}{|llr|}
\hline SRM & \multicolumn{1}{c}{ Type } & $\mathrm{n}^{20}$ \\
\hline $211 \mathrm{c}$ & Toluene & 1.497 \\
\hline $217 \mathrm{c}$ & $2,2,4-$ Trimethylpentane & 1.391 \\
\hline 1820 & Glass (Borosilicate) & 1.488 \\
\hline 1822 & Glass (Soda-Lime) & 1.518 \\
\hline $1823-\mathrm{I}$ & Silicone Liquid (I) & 1.518 \\
\hline $1823-$ II & Silicone Liquid (II) & 1.559 \\
\hline
\end{tabular}

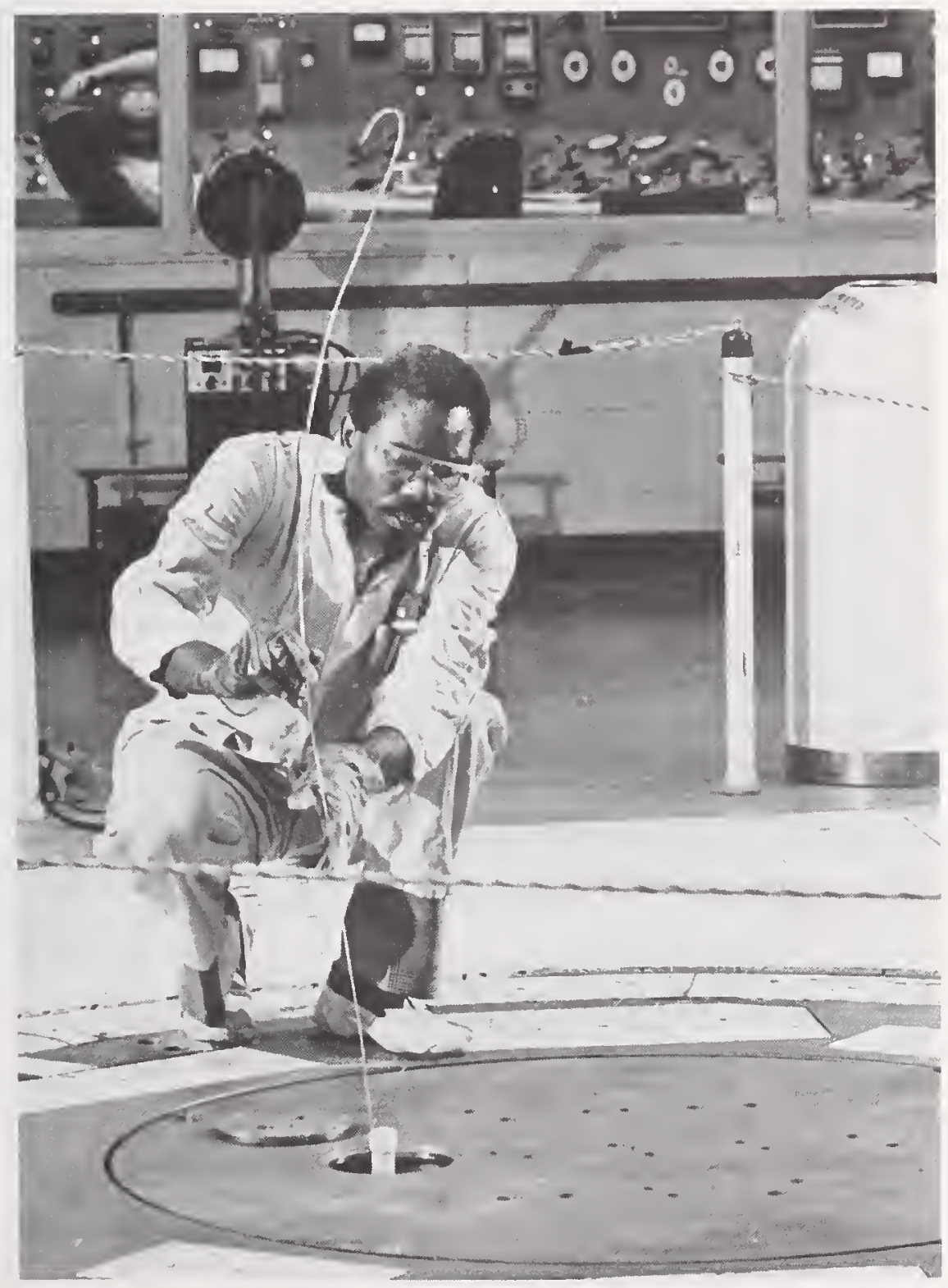

NBS uses its research reactor for a variety of research work, including the analysis of environmental and biological SRM's. Thomas Gills inserts a sample into the reactor. 


\section{Optical Rotation}

These SRM's are intended for use in calibrating or checking polarimetric apparatus. In aqueous solution the optical rotation of SRM $17 \mathrm{c}$ is certified at three wavelengths, while that of SRM $41 \mathrm{~b}$ is certified at two wavelengths. SRM $41 \mathrm{~b}$ is also certified at one wavelength in a dimethyl sulfoxide solution.

\begin{tabular}{|llcc|}
\hline SRM & Type & Optical Rotation In Aqueous Solution & Unit Size \\
\hline $17 \mathrm{c}$ & Sucrose & $712 \mathrm{mrad}$ & $60 \mathrm{~g}$ \\
\hline $41 \mathrm{~b}$ & Dextrose & $1091 \mathrm{mrad}$ & $70 \mathrm{~g}$ \\
\hline
\end{tabular}

\section{Radionctivity}

Information regarding radioactivity SRM's appears on it or its container. A Certificate containing pertinent information on the SRM is sent under separate cover; a photocopy of the certificate is sent with the SRM. Copies of these Certificates and information concerning the applications of these SRM's are available on request to the NBS Office of Standard Reference Materials. These materials are shipped only by express or air freight (shipping charges collect). The prices of SRM's may change as current stocks are depleted and are replaced. Purchasers will be billed at the prices in effect at the time of shipment.

The stated uncertainties of the older standards are, in general, an estimate of the standard deviation added to an estimate of maximum possible systematic error. The total uncertainties for more recent standards are based on the 99-percent confidence interval for the random uncertainty, with the same estimate of systematic error.

The amount of a radionuclide in an SRM, at a specified time, is stated as (1) the number of atoms (or the mass, for radium standards), (2) the activity, or "decays per second", or (3) the emission rate of a particular radiation, depending on the method of calibration or the intended use. For solution standards, the quantity is usually specified per gram of liquid, with the supposition that users will gravimetrically dispense or dilute the material to duplicate their usual counting conditions. The active portion of gamma-ray "pointsource" standards is usually restricted to the central few millimeters of a low-mass, low-Z support in order to minimize scattering. Standards of alpha-particle-emitting radionuclides are deposited or plated on metal backings.

The unit for activity has traditionally been the curie $(\mathrm{Ci})$, but simpler relations between activity, emission rate, and counting rate result if the current SI (International System of Units) unit " 1 per second" is used. This is symbolized as " $\mathrm{s}^{-1}$ " and has been given the special name becquerel (Bq). The relationship between the curie and the becquerel is $1 \mathrm{Ci}=3.7 \times 10^{10} \mathrm{~Bq}$.

Many SRM's are measured and certified in terms of emission rate. If the fraction of radioactive decays which give rise to a specific radiation is known, the emission rate for that radiation can be deduced from the measured activity, and conversely, the activity may be deduced from the measured emission rate. Suggested values for probabilities per decay for the more intense radiations usually accompany each SRM, or can be obtained from the Radioactivity Group. In this catalog $\alpha \mathrm{s}^{-1}, \beta^{-} \mathrm{s}^{-1}, \beta^{+} \mathrm{s}^{-1}, \mathrm{Kxs}^{-1}$, and $\gamma \mathrm{s}^{-1}$ are used for the emission rates of alpha particles, negatrons, positrons, $\mathrm{K}$ x-rays, and gamma rays, respectively.

The SRM's listed below, not marked with an asterisk $\left(^{*}\right)$, may be ordered singly, without a license, under the general licensing provisions of the Atomic Energy Act of 1954. Those marked by an asterisk are available only under the special licensing provisions of the Atomic Energy Act of 1954.

Note: Certain radionuclides are not economical to maintain in stock because of short half lives or low demand. When sufficient demand exists, based on letters of inquiry, these materials are prepared and those 
who have expressed interest are notified of their availability. If you need any radionuclides not listed, contact the Radioactivity Section, Room C114, Radiation Physics Building, National Bureau of Standards,

Washington, DC 20234 (Telephone: 301-921-2665).

In addition, chemically stable solutions of most radionuclides, including those no longer issued by NBS or that are currently out of stock, may be sumbitted to NBS for calibration as described in "Calibration and Related Measurement Services of the National Bureau of Standards," NBS Special Publication 250 (1980). Requests for these tests should be submitted, with full source information for approval of suitability, to the Radioactivity Section.

\begin{tabular}{|c|c|c|c|c|c|}
\hline \multirow{2}{*}{$\frac{\text { SRM }}{4229}$} & Radionuclide & \multicolumn{2}{|c|}{$\begin{array}{c}\text { Approximate activity, per gram, } \\
\text { at time of calibration (month/year) } \\
\left(\left(\mathrm{Bq} \mathrm{s}^{-1}\right)\right.\end{array}$} & \multirow{2}{*}{$\begin{array}{c}\text { Approx. Mass of } \\
\begin{array}{c}\text { Solution } \\
\text { (g) }\end{array} \\
4.6\end{array}$} & \multirow{2}{*}{$\begin{array}{c}\begin{array}{c}\text { Overall } \\
\text { Uncertainty } \\
(\%)\end{array} \\
\pm 1.1\end{array}$} \\
\hline & Aluminum-26 & 39 & $11 / 71$ & & \\
\hline $4251 \mathrm{~B}^{*}$ & Barium-133 & $5 \times 10^{5}$ & $1 / 82$ & 5 & 1.4 \\
\hline 4245 & Carbon-14 & $4 \times 10^{5}$ & $5 / 74$ & 5 & 1.0 \\
\hline 4246 & Carbon-14 & $4 \times 10^{4}$ & $5 / 74$ & 5 & 0.9 \\
\hline $4250 \mathrm{~B}^{*}$ & Cesium-134 & $2 \times 10^{6}$ & $4 / 82$ & 5 & 1.2 \\
\hline $4233 \mathrm{~B}^{*}$ & Cesium-137, Barium-137 m & $7 \times 10^{5}$ & $8 / 79$ & 5.1 & 1.4 \\
\hline 4943 & Chlorine-36 & $1 \times 10^{4}$ & $4 / 62$ & 3 & 2.3 \\
\hline $4422 \mathrm{~L}^{*}$ & Chlorine-36 & $4 \times 10^{4}$ & $4 / 80$ & 5.1 & 1.6 \\
\hline $4915 D^{*}$ & Cobalt-60 & $6 \times 10^{5}$ & $5 / 80$ & 5 & 0.7 \\
\hline $4370 B^{*}$ & Europium-152 & $2 \times 10^{5}$ & $6 / 79$ & 5 & 1.5 \\
\hline $4926 \mathrm{C}$ & Hydrogen-3 & $3 \times 10^{3}$ & $9 / 78$ & 18 & 0.6 \\
\hline 4947 & Hydrogen-3 & $1 \times 10^{5}$ & $9 / 78$ & 4 & 1.0 \\
\hline 4361 & Hydrogen-3 & 1.3 & $9 / 78$ & 490 & 0.9 \\
\hline $4949 \mathrm{~B}$ & Iodine- 129 & $7 \times 10^{3}$ & $1 / 82$ & 1 & 1.9 \\
\hline $4257^{*}$ & Manganese-54 & $6 \times 10^{5}$ & $4 / 79$ & 5 & 1.0 \\
\hline $4226^{*}$ & Nickel-63 & $2 \times 10^{6}$ & $5 / 68$ & 4.1 & 1.0 \\
\hline $4331 *$ & Plutonium-239 & $6 \mathrm{~s}^{-1} \mathrm{~g}^{-1}$ & $3 / 75$ & 2 & 1.0 \\
\hline $4338^{*}$ & Plutonium-240 & $18 \mathrm{~s}^{-1} \mathrm{~g}^{-1}$ & $4 / 80$ & 5 & 1.0 \\
\hline $4334 \mathrm{~B}^{*}$ & Plutonium-242 & $27 \mathrm{~s}^{-1} \mathrm{~g}^{-1}$ & $5 / 79$ & 5 & 0.9 \\
\hline $4945 D^{*}$ & Strontium-89 & $5 \times 10^{4}$ & $11 / 82$ & 5 & 1.5 \\
\hline $4288^{*}$ & Technetium-99 & $4 \times 10^{4}$ & $11 / 82$ & 5 & 1.6 \\
\hline \multicolumn{6}{|c|}{ Long-Lived Mixed Radionuclide: } \\
\hline \multirow[t]{3}{*}{$4276 \mathrm{~B}^{*}$} & Antimony-125 & $1.2 \times 10^{4}$ & $6 / 83$ & 5 & \\
\hline & Europium-154 & $1.5 \times 10^{4}$ & $6 / 83$ & & \\
\hline & Europium-155 & $7 \times 10^{3}$ & $6 / 83$ & & \\
\hline
\end{tabular}

* License certification is required by NBS for these radionuclides. 


\section{Alpha-Particle Point-Source}

These SRM's consist of a practically weightless deposit of the nuclide on a thin platinum foil cemented to a monel disk.

\begin{tabular}{|c|c|c|c|c|}
\hline \multirow{2}{*}{$\begin{array}{l}\text { SRM } \\
4904 F^{*}\end{array}$} & \multirow{2}{*}{$\begin{array}{l}\text { Radionuclide } \\
\text { Americium-241 }\end{array}$} & \multicolumn{2}{|c|}{$\begin{array}{l}\text { Approx. } \alpha \text {-particle-emission rate into } 2 \pi \\
\text { geometry and/or approx. activity at time } \\
\text { of calibration (month/year) }\end{array}$} & \multirow{2}{*}{$\frac{\begin{array}{c}\text { Overall uncertainty } \\
(\%)\end{array}}{1.0 \text { to } 1.3}$} \\
\hline & & 30 to $1.3 \times 10^{4} \mathrm{~s}^{-1}$ & $2 / 82$ & \\
\hline $4907^{*}$ & Gadolinium-148 & $6 \times 10^{2}$ to $3 \times 10^{4} s^{-1}$ & $8 / 79$ & 0.7 to 1.6 \\
\hline $4906 \mathrm{~B}$ & Plutonium-238 & $4 \times 10^{2}$ to $4 \times 10^{4} \mathrm{~s}^{-1}$ & $7 / 78$ & 0.7 to 2.2 \\
\hline
\end{tabular}

*License certification is required by NBS for these radionuclides.

\section{Radiocarbon Dating and Ground Water Studies}

\section{Contemporary Standard for Carbon-14 Dating Laboratories}

\begin{tabular}{|lll|}
\hline SRM & Material & \multicolumn{1}{c|}{ Description } \\
\hline $4990 \mathrm{C}$ & Oxalic Acid & $\begin{array}{l}\text { One-half pound of oxalic acid taken from specially prepared material } \\
\text { for use as a common contemporary standard against which world- } \\
\text { wide measurements can be compared. }\end{array}$ \\
& &
\end{tabular}

\section{Low-Level Tritiated-Water Standard}

\begin{tabular}{|lll|}
\hline SRM & Material & Description \\
\hline 4361 & Hydrogen-3 & $\begin{array}{l}\text { Contains } 490 \text { grams of }{ }^{3} \mathrm{H}-\mathrm{H}_{2} \mathrm{O} \text { in a flame-sealed bottle. The } \\
\text { radioactivity concentration was } 1.312 \mathrm{~Bq} \mathrm{~g}^{-1}, \text { as of the } \\
\text { date of the most recent gas-counting measurement-September } 3, \\
1978 . \text { The total uncertainty in this value is } 0.85 \% .\end{array}$ \\
\hline
\end{tabular}

Melody Smith calibrates SRM 930D, Glass Filters for Spectrophotometry. This SRM is a reference source for the verification of the transmiltance and absorbance scales of spectrophotometers.

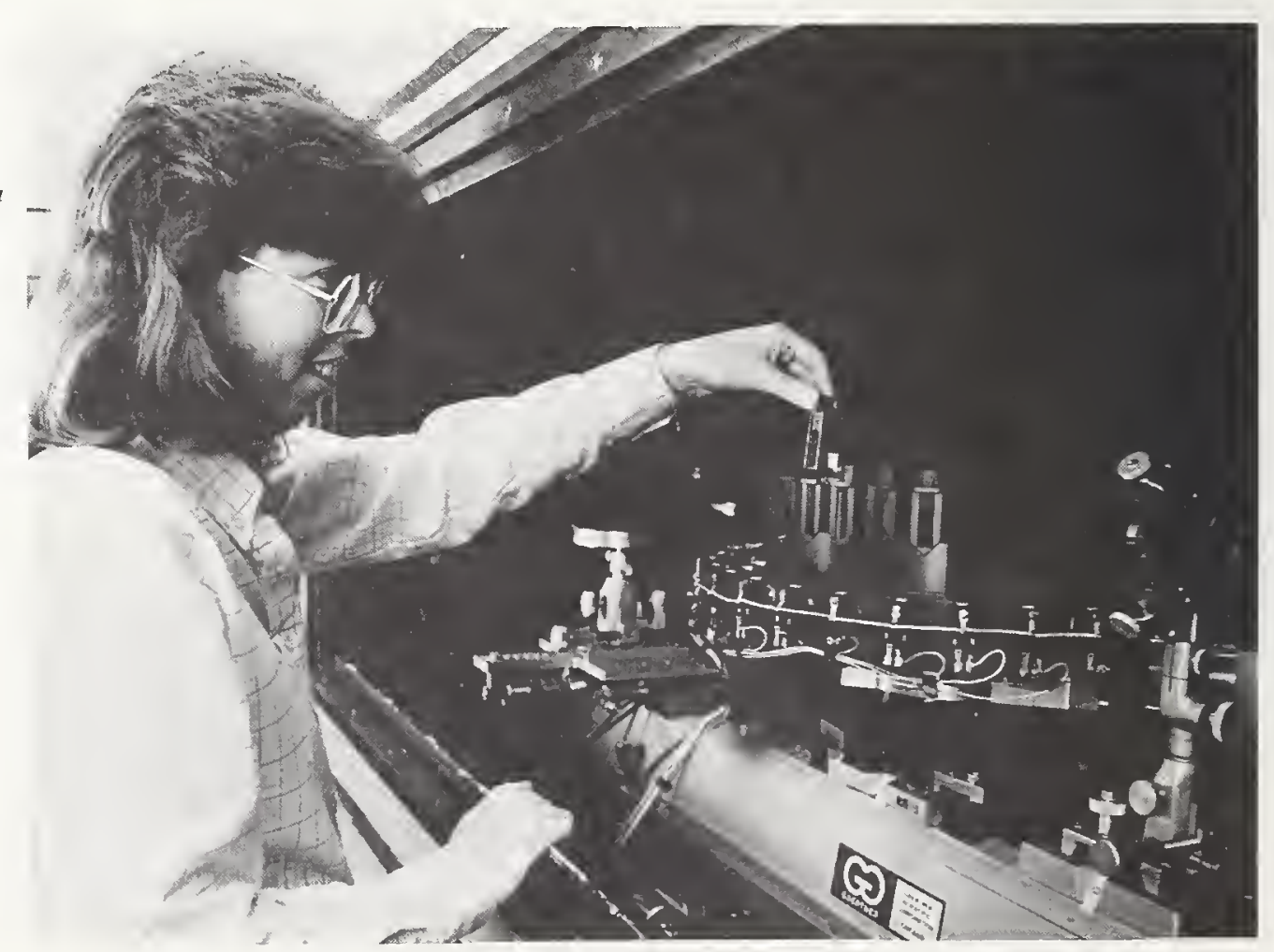




\section{Gaseous Radioactivity}

\begin{tabular}{|c|c|c|c|c|c|c|}
\hline SRM & Radionuclide & $\begin{array}{l}\text { Approximate } \\
\text { concentration at ti }\end{array}$ & $\begin{array}{l}\text { ty } \\
\text { th/year) }\end{array}$ & $\begin{array}{l}\text { Approx. } \\
\text { Vol, } \\
\left(\mathrm{cm}^{3}\right)\end{array}$ & $\begin{array}{l}\text { Approx. } \\
\text { Pressure } \\
\quad \text { (atm) }\end{array}$ & $\begin{array}{c}\text { Overall } \\
\text { Uncertainty } \\
(\%)\end{array}$ \\
\hline $4935 \mathrm{C}$ & Krypton-85 & $5 \times 10^{7} \mathrm{~s}^{-1} \mathrm{~mol}^{-1}$ & $3 / 74$ & 10 & 1 & \pm 0.9 \\
\hline $4235^{*}$ & Krypton- 85 & $1 \times 10^{7} \mathrm{~s}^{-1}$ & $11 / 74$ & 3 & 1 & 1.2 \\
\hline $4308 \mathrm{C}$ & Krypton-85 & $1.6 \times 10^{6} \mathrm{~s}^{-1}$ & $11 / 79$ & 30 & 0.3 & 3.1 \\
\hline $4309 \mathrm{G}^{*}$ & Xenon-127 & $3 \times 10^{6} s^{-1}$ & $11 / 82$ & 30 & 0.3 & 2.1 \\
\hline$\overline{4307 I^{*}}$ & Xenon-133 & $2 \times 10^{6} \mathrm{~s}^{-1}$ & $\begin{array}{l}\text { time of } \\
\text { dispatch }\end{array}$ & 30 & 0.3 & 2.2 \\
\hline$\overline{4415 \mathrm{LI}^{*}}$ & Xenon-133 & $1.5 \times 10^{8} \mathrm{~s}^{-1}$ & $\begin{array}{l}\text { time of } \\
\text { dispatch }\end{array}$ & 5 & 0.1 & 1.5 \\
\hline \multicolumn{7}{|c|}{ Mixed-Radionuclide Gas: } \\
\hline \multirow[t]{3}{*}{$4310 \mathrm{~B}^{*}$} & Krypton-85 & & & 30 & 1 & 2.5 \\
\hline & Xenon-127 & & & & & 1.7 \\
\hline & Xenon-133 & & & & & 1.6 \\
\hline
\end{tabular}

${ }^{*}$ License certification is required by NBS for these radionuclides.

\section{Gamma-Ray and X-Ray Point-Source}

This group of Standard Reference Materials is usually prepared by depositing the radioactive material and sealing it between two layers of polyester tape, mounted on an aluminum ring. Exceptions to this procedure are americium, and thorium SRM's. SRM 4213, americium-241 is prepared by electroplating americium onto a $0.010-\mathrm{cm}$ thick platinum foil, which is covered with a $0.005-\mathrm{cm}$ thick aluminum foil. The aluminum-covered source is sandwiched between two layers of $0.036-\mathrm{cm}$ thick polyurethane film tape. SRM 4206c, thorium-228, is prepared by depositing and sealing the radionuclide between two layers of gold foil and this sandwich is then sealed between two double layers of polyurethane-film tape.

\begin{tabular}{|c|c|c|c|c|c|}
\hline \multirow{2}{*}{$\frac{\text { SRM }}{4213^{*}}$} & \multirow{2}{*}{$\frac{\text { Radionuclide }}{\text { Americium-24l }}$} & \multirow{2}{*}{$\begin{array}{c}\begin{array}{c}\text { Principal } \\
\text { Photon Energy } \\
(\mathrm{MeV})\end{array} \\
0.060\end{array}$} & \multicolumn{2}{|c|}{$\begin{array}{c}\text { Approximate activity, } \mathrm{s}^{-1} \text {, at time } \\
\text { of calibration (except MRN) } \\
\text { (month/year) }\end{array}$} & \multirow{2}{*}{$\begin{array}{c}\begin{array}{c}\text { Overall } \\
\text { Uncertainty } \\
(\%)\end{array} \\
\pm 2.8 \\
\end{array}$} \\
\hline & & & $3 \times 10^{5}$ & $2 / 70$ & \\
\hline $4241 B^{*}$ & Barium-133 & 0.081 & $8 \times 10^{4}$ & $1 / 81$ & 1.4 \\
\hline $4200 \mathrm{~B}$ & Cesium-137, Barium-137m & 0.662 & $4 \times 10^{4}$ & $9 / 79$ & 1.6 \\
\hline 4207 & Cesium-137, Barium-137m & 0.662 & $3 \times 10^{5}$ & $9 / 79$ & 1.6 \\
\hline $4218 \mathrm{E}^{*}$ & Europium-152 & 0.122 to 1.408 & $5 \times 10^{4}$ to $5 \times 10^{5}$ & $11 / 82$ & 1.5 \\
\hline $4997 \mathrm{E}$ & Manganese-54 & 0.835 & $3 \times 10^{5}$ & $4 / 79$ & 1.0 \\
\hline $4201 \mathrm{~B}$ & Niobium-94 & 0.702 to 0.871 & $5 \times 10^{3}$ & $4 / 70$ & 1.5 \\
\hline $4206 C^{*}$ & Thorium-228 & 2.615 & $8 \times 10^{4}$ & $11 / 80$ & 2.0 \\
\hline \multicolumn{6}{|c|}{ Long-Lived Mixed Radionuclide: } \\
\hline \multirow[t]{3}{*}{$4275 \mathrm{~B}$} & Antimony-125-Tellurium- $125 \mathrm{~m}$, & 0.027 to 1.274 & $5 \times 10^{4}$ & $9 / 80$ & \\
\hline & Europium-154 & & $6 \times 10^{4}$ & & \\
\hline & Europium-155 & & $3 \times 10^{4}$ & & \\
\hline
\end{tabular}

* License certification is required by NBS for these radionuclides. 


\section{Low-Energy-Photon Point-Source}

These SRM's consist of a thin-layer deposit of the radionuclide on a thin stainless steel or platinum foil cemented to a monel disk.

\begin{tabular}{|c|c|c|c|c|c|}
\hline SRM & Radionuclide & $\begin{array}{c}\text { Principal } \\
\text { Photon Energy } \\
(\mathrm{MeV})\end{array}$ & $\begin{array}{l}\text { Approx. emission } \\
\text { of calibration (mo }\end{array}$ & $\begin{array}{l}\text { t time } \\
\text { ear) }\end{array}$ & $\begin{array}{c}\text { Overall } \\
\text { Uncertainty } \\
(\%)\end{array}$ \\
\hline \multirow[t]{2}{*}{4266} & Vanadium -49 & 0.0045 & $2 \times 10^{4}$ & $2 / 81$ & \pm 2.3 \\
\hline & & & $\mathrm{K}-\times \mathrm{s}^{-1}$ steradian $^{-1}$ & & \\
\hline \multirow[t]{2}{*}{$4260 \mathrm{C}$} & Iron-55 & 0.0059 & $2 \times 10^{4}$ & $11 / 82$ & 1.8 \\
\hline & & & $\mathrm{K}-\times \mathrm{s}^{-1}$ steradian $^{-1}$ & & \\
\hline $4264 \mathrm{~B}$ & Tin-121m, Antimony-121 & 0.0372 & $5 \times 10^{2} \mathrm{~s}^{-1}$ & $11 / 82$ & 3.0 \\
\hline
\end{tabular}

\section{Radium-226 Solution}

\section{Radon Analysis}

These samples are contained in flame-sealed glass ampoules.

\begin{tabular}{|c|c|c|c|c|}
\hline SRM & \multicolumn{2}{|c|}{ Nominal Radium Content (g) (month/year) } & $\begin{array}{l}\text { Approx. Mass } \\
\text { of Solution } \\
(\mathrm{g})\end{array}$ & $\begin{array}{c}\text { Overall } \\
\text { Uncertainty } \\
(\%)\end{array}$ \\
\hline $4952 B$ & Blank Solution & $8 / 76$ & 20 & \pm 68 \\
\hline$\overline{4953 \mathrm{C}}$ & $10^{-8}$ & $4 / 78$ & 10.3 & 1.3 \\
\hline
\end{tabular}

\section{Gamma-Ray Solution}

These samples are contained in flame-sealed glass ampoules.

\begin{tabular}{|c|c|c|c|c|}
\hline SRM & Nominal & ear) & $\begin{array}{l}\text { Approx. Mass } \\
\text { of Solution } \\
\text { (g) }\end{array}$ & $\begin{array}{c}\text { Overall } \\
\text { Uncertainty } \\
(\%)\end{array}$ \\
\hline 4956 & $2 \times 10^{-7}$ & $9 / 67$ & 5.1 & \pm 4.4 \\
\hline 4957 & $5 \times 10^{-7}$ & $9 / 67$ & 5.1 & 1.8 \\
\hline 4958 & $1 \times 10^{-6}$ & $9 / 67$ & 5.1 & 1.8 \\
\hline 4959 & $2 \times 10^{-6}$ & $9 / 67$ & 5.1 & 1.3 \\
\hline 4960 & $5 \times 10^{-6}$ & $9 / 67$ & 5.1 & 1.3 \\
\hline 4961 & $1 \times 10^{-5}$ & $9 / 67$ & 5.1 & 1.1 \\
\hline 4962 & $2 \times 10^{-5}$ & $9 / 67$ & 5.1 & 1.1 \\
\hline 4963 & $5 \times 10^{-5}$ & $9 / 67$ & 5.1 & 1.1 \\
\hline $4964 \mathrm{~B}$ & $1 \times 10^{-4}$ & $6 / 65$ & 5.2 & 0.5 \\
\hline
\end{tabular}




\section{Environmental Natural Matrix Standards for Traceability Tests}

\section{SRM 435OB-Columbia River Sediment}

This material was collected from a river downstream from a nuclear reactor facility. Concentrations of fission and activation products are elevated over typical world-wide levels. ${ }^{239 / 240} \mathrm{Pu}$ and ${ }^{241} \mathrm{Am}$ are very

homogeneously distributed through the sample and are in soluble chemical forms. Inhomogeneity is 3 percent or better for other radionuclides.

\section{SRM 4351-Human Lung}

This material contains radioactivity concentrations on the order of $10^{-4} \mathrm{~Bq} \mathrm{~g}^{-1}$. It has been freeze-dried, cryogenically ground, homogenized, and packed in a glass bottle under vacuum. There is significant inhomogeneity in ${ }^{239 / 240} \mathrm{Pu}$ which is unavoidable because plutonium was taken into the lungs in particulate form. Assessments of accuracy of measurement technique can be improved by averaging over several samples.

\section{SRM 4352-Human Liver}

This material contains radioactivity concentrations on the order of $10^{-4} \mathrm{~Bq} \mathrm{~g}^{-1}$. It has been freeze-dried, cryogenically ground, homogenized, and packed in a glass bottle under vacuum.

\section{SRM 4353-Rocky Flats Soil Number 1}

This material was collected within 13 centimeters of the soil surface at Rock Flats, $\mathrm{CO} .{ }^{239} \mathrm{Pu}$ and ${ }^{241} \mathrm{Am}$ concentrations are about an order of magnitude higher than typical world-wide levels. Approximately 10 percent of the plutonium is in a refractory chemical state. The material also contains "hot" particles and a statistical method is provided for dealing with these. Inhomogeneities, excluding hot particles, are on the order of 3 percent or better.

\section{SRM 4355-Peruvian Soil}

This material, which has been the subject of a trace-element study, has non-measurable radioactivity concentrations for many fallout radionuclides and can be used as a blank or for sensitive tests of radioanalytical procedures at low-radioactivity concentrations for other radionuclides. The results of the trace-element study are given for 57 elements.

\section{RM 45B-River Sediment}

This material contains radioactivity concentrations of roughly an order of magnitude greater than SRM $435 \mathrm{OB}$. The values, however, are uncertified although the inhomogeneity is better than 3 percent for all radionuclides. This material can be used for routine checking for reproducibility of results after tests have been performed with SRM 435OB.

\section{Radiopharmaceutical}

\begin{tabular}{|c|c|c|c|c|c|}
\hline SRM & Radionuclide & $\begin{array}{l}\text { Half } \\
\text { Life }\end{array}$ & & $\begin{array}{l}\text { Approximate Radioactivity } \\
\text { at Time of Dispatch }\end{array}$ & $\begin{array}{c}\text { Overall } \\
\text { Uncertainty }\end{array}$ \\
\hline 4400LF* & Chromium-51 & 27.704 & $\mathrm{~d}$ & $1 \times 10^{6}$ & \pm 1.8 \\
\hline $4408 \mathrm{LC}^{*}$ & Cobalt-57 & 270.9 & $\mathrm{~d}$ & $8 \times 10^{5}$ & 1.6 \\
\hline $4416 \mathrm{LD}^{*}$ & Gallium-67 & 78.26 & $\mathrm{hr}$ & $3 \times 10^{6}$ & 1.7 \\
\hline $4421 \mathrm{~L}^{*}$ & Gold-195 & 183 & $\mathrm{~d}$ & $5 \times 10^{5}$ & 2.3 \\
\hline $4405 \mathrm{LB}^{*}$ & Gold-198 & 2.696 & $\mathrm{~d}$ & $4 \times 10^{6}$ & 1.7 \\
\hline $4417 \mathrm{LC}^{*}$ & Indium-111 & 2.83 & $\mathrm{~d}$ & $3 \times 10^{6}$ & 1.3 \\
\hline $4414 \mathrm{LC}^{*}$ & Iodine -123 & 13.2 & $\mathrm{hr}$ & $6 \times 10^{7}$ & 1.5 \\
\hline $4407 \mathrm{LH}^{*}$ & Iodine- 125 & 60.14 & $\mathrm{~d}$ & $8 \times 10^{5}$ & 2.0 \\
\hline 4401 LI* $^{*}$ & Iodine-131 & 8.04 & $\mathrm{~d}$ & $1 \times 10^{6}$ & 1.7 \\
\hline $4411 \mathrm{LB}^{*}$ & Iron-59 & 44.529 & $\mathrm{~d}$ & $8 \times 10^{5}$ & 1.5 \\
\hline $4420 \mathrm{~L}^{*}$ & Lead-203 & 52.05 & $\mathrm{hr}$ & $3 \times 10^{6}$ & 1.7 \\
\hline $4418 \mathrm{~L}^{*}$ & Mercury-203 & 46.60 & $\mathrm{~d}$ & $1 \times 10^{6}$ & 1.0 \\
\hline $4412 \mathrm{LH}^{*}$ & Molybdenum-99, Technetium-99m & 66.0 & $\mathrm{hr}$ & $2 \times 10^{6}$ & 1.8 \\
\hline $4406 \mathrm{LG}^{*}$ & Phosphorus-32 & 14.29 & $\mathrm{~d}$ & $1 \times 10^{6}$ & 1.4 \\
\hline 4409 LD* $^{*}$ & Selenium-75 & 119.8 & $\mathrm{~d}$ & $1 \times 10^{6}$ & 2.5 \\
\hline
\end{tabular}




\section{Radiopharmaceutical (Continued)}

\begin{tabular}{|llcccc|}
\hline SRM & \multicolumn{1}{c}{ Radionuclide } & $\begin{array}{c}\text { Half } \\
\text { Life }\end{array}$ & $\begin{array}{c}\text { Approximate Radioactivity } \\
\text { at Time of Dispatch }\end{array}$ & $\begin{array}{c}\text { Overall } \\
\text { Uncertainty }\end{array}$ \\
\hline $4403 \mathrm{LB}^{*}$ & Strontium-85 & 64.84 & $\mathrm{~d}$ & $1 \times 10^{6}$ & 1.4 \\
\hline $4410 \mathrm{HI}^{*}$ & Technetium-99m & 6.02 & $\mathrm{hr}$ & $2 \times 10^{9}$ & 1.8 \\
\hline $4404 \mathrm{LF}^{*}$ & Thallium-201 & 3.044 & $\mathrm{~d}$ & $2 \times 10^{6}$ & 2.0 \\
\hline $4402 \mathrm{LC}^{*}$ & Tin-113, Indium-113m & 115.1 & $\mathrm{~d}$ & $8 \times 10^{5}$ & 3.1 \\
\hline $4415 \mathrm{LI}^{*}$ & Xenon-133 & 5.245 & $\mathrm{~d}$ & $5 \times 10^{8} \mathrm{~s}^{-1}$ total & 1.4 \\
\hline $4419 \mathrm{LB}^{*}$ & Ytterbium-169 & 32.01 & $\mathrm{~d}$ & $2 \times 10^{6}$ & 2.5 \\
\hline
\end{tabular}

*License certification is required by NBS for these radionuclides.

\section{Special Nuclear Material Packaging}

\begin{tabular}{|ccc|}
\hline SRM & Type & Description \\
\hline 9910 & Special Nuclear Material Package & Lead Container, $21 / 2 \times 6$ inches \\
\hline
\end{tabular}

NOTE: This material may be necessary to fill your order, depending upon Department of Energy, Department of Transportation, and Nuclear Regulatory Commission shipping requirements.

\section{Catalyst Package for Lubrifant Oxidation}

SRM 1817 is intended primarily for use in evaluating the oxidation stability of lubricating oils, i.e., automotive crankcase lubricants. The SRM contains: (1) an oxidized/mitrated fuel fraction, (2) a metal naphthenate mixture, and (3) distilled water. The metal naphthenate mixture has the following weight percentages of metal naphthenates: lead-82, iron-7, copper-4, manganese-3.5, and tin-3.5. SRM 1817 is available as a kit of 5 ampoules of each of the three components. The fuel and metal catalysts are sealed under inert atmosphere to ensure their stabilities.

\section{Metallurgicall}

SRM 493 is intended for calibration x-ray diffraction equipment to determine the relative amounts of iron carbide in steel. SRM's 485a, 486, 487, and 488 are intended for the calibration of x-ray diffraction equipment used in determining the amount of reatined austenite in ferrous materials.

\begin{tabular}{|llll|}
\hline SRM & \multicolumn{2}{c}{ Type } & Form \\
\hline $485 \mathrm{a}$ & Austenite in Ferrite & $5 \%$ & Disk \\
\hline 486 & Austenite in Ferrite & $15 \%$ & Disk \\
\hline 487 & Austenite in Ferrite & $30 \%$ & Disk \\
\hline 488 & Austenite in Ferrite & $2 \%$ & Disk \\
\hline 493 & Spheroidized Iron Carbide $\left(\mathrm{Fe}_{3} \mathrm{C}\right)$ & in Ferrite & Wafer: $29 \times 29 \times 2.4 \mathrm{~mm}$ \\
\hline
\end{tabular}

\section{Mo̊ssbauer}

These SRM's are issued for the calibration of the isomer shift of iron compounds and alloys and to provide a uniform basis for presentation of Mössbauer isomer shift data.

\begin{tabular}{|c|c|c|}
\hline SRM & Type & Form \\
\hline 493 & Spheroidized Iron Carbide $\left(\mathrm{Fe}_{3} \mathrm{C}\right)$ in Ferrite & Wafer: $29 \times 29 \times 2.4 \mathrm{~mm}$ \\
\hline 1541 & Iron Foil & Foil: $2.5 \mathrm{~cm} \times 2.5 \mathrm{~cm} \times 23 \mu \mathrm{m}$ \\
\hline
\end{tabular}


SRM 1857 is a D.2 tool steel block that is issued for use in the dry sand/rubber wheel abrasion test according to ASTM G65, Procedure A.

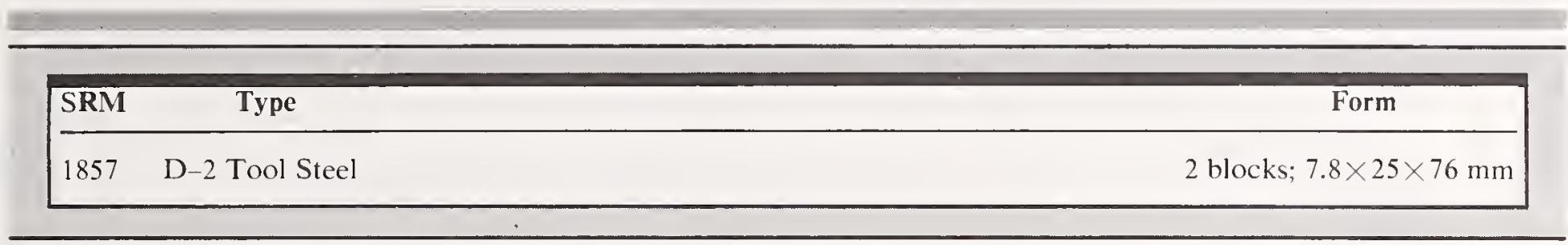

\section{Pitting or Crevice Corrosion}

SRM 1890 is a $316 \mathrm{~L}$ stainles steel rod that is issued for use in evaluating the pitting or crevice corrosion of surgical implant materials according to ASTM F746.

\begin{tabular}{|lcr|}
\hline SRM & Type & Form \\
\hline 1890 & $316 \mathrm{~L}$ Stainless Steel Rod and Teflon Collar & 4 sets; $6.4 \mathrm{~mm}$ D, 25.4 mm long \\
\hline
\end{tabular}

\section{X-ray Fluorescent Emission Target:}

This SRM is intended for use in determining the detector window absorption in semiconductor $\mathrm{x}$-ray spectrometers according to ANSI-IEEE Standard STO 759. When excited by a ${ }^{55}$ Fe source this glass target will emit fluorescent $\mathrm{x}$-rays in the range 1.0 to $5.2 \mathrm{keV}$.

\begin{tabular}{|lcrrr|}
\hline SRM & Type & Form & Unit Size \\
\hline 477 & Glass Fluorescence Source & Disk & $2 \times 25 \mathrm{~mm}$ \\
\hline
\end{tabular}

\section{X-ray Diffraction}

SRM's 640a, 674, and 675 are powdered materials to be used as internal standards for powder diffraction measurements. The lattice parameter of SRM 640a has been accurately determined at $25.0{ }^{\circ} \mathrm{C}$ using a high angle goniometer and the NBS tungsten and silver internal standards. The weighted average of the lattice parameter, uncorrected for refraction, is $5.430825 \AA$. SRM 674 is a set of five oxides for use in the quantitative analysis (intensity measurement) of materials. SRM 675 (synthetic fluorophlogopite) is a large d-spacing standard. The lattice parameter is $9.98104 \AA$.

\begin{tabular}{|c|c|c|}
\hline SRM & Type & Unit Size \\
\hline $640 \mathrm{a}$ & Silicon Powder & $10 \mathrm{~g}$ \\
\hline \multirow[t]{6}{*}{674} & Powder Diffraction Intensity & \\
\hline & $\mathrm{Al}_{2} \mathrm{O}_{3}(\alpha$-alumina $)$ & $10 \mathrm{~g}$ \\
\hline & $\mathrm{CeO}_{2}$ & $10 \mathrm{~g}$ \\
\hline & $\mathrm{Cr}_{2} \mathrm{O}_{3}$ & $10 \mathrm{~g}$ \\
\hline & $\mathrm{TiO}_{2}$ (rutile) & $10 \mathrm{~g}$ \\
\hline & $\mathrm{ZnO}$ & $10 \mathrm{~g}$ \\
\hline 675 & Powder Diffraction (Mica) & $5 \mathrm{~g}$ \\
\hline
\end{tabular}




\section{Gas Transmission}

SRM 1470 is for use in the measurement of gas transmission rates using a volumetric method (ASTM D1434), manometric method (ASTM D1434), or coulometric method (ASTM D3985) of measurement. The permeances of nitrogen, oxygen, carbon dioxide, and helium through this polyester film at $296.15 \mathrm{~K}$ are $0.0421,0.352,1.722$, and $13.79 \mathrm{pmol} \cdot \mathrm{s}^{-1} \cdot \mathrm{Pa}^{-1}$, respectively.

\begin{tabular}{|ccc|}
\hline SRM & Type & Unit Size \\
\hline 1470 & Polyester Plastic Film for Gas Transmission $\left(\mathrm{CO}_{2}, \mathrm{He}, \mathrm{N}_{2}\right.$, and $\left.\mathrm{O}_{2}\right)$ & 15 sheets, $23 \mathrm{~cm}$ square \\
\hline
\end{tabular}

\section{Reference Fuel}

SRM's 1815 and 1816 are high purity liquids intended for use in maintaining the integrity of the octane rating of motor and aviation fuels as specified in the ASTM Manual for Rating Motor, Diesel and Aviation Fuels.

\section{Electrical Resistivity and Conductivity}

\section{Metals}

These SRM's are useful in evaluating methods of measuring electrical resistance over wide temperature ranges.

\begin{tabular}{|c|c|c|c|c|}
\hline SRM & Type & Temperature Range & Resistivity at $293 \mathrm{~K}$ & Form \\
\hline 1460 & Stainless Steel & 5 to $1200 \mathrm{~K}$ & $80.5 \mu \Omega \cdot \mathrm{cm}$ & Rod; $\quad 6.4 \mathrm{~mm} \mathrm{D}, 50 \mathrm{~mm}$ long \\
\hline 1461 & Stainless Steel & 5 to $1200 \mathrm{~K}$ & $80.5 \mu \Omega \cdot \mathrm{cm}$ & Rod; $12.7 \mathrm{~mm} \mathrm{D,} 50 \mathrm{~mm}$ long \\
\hline 1462 & Stainless Steel & 5 to $1200 \mathrm{~K}$ & $80.5 \mu \Omega \cdot \mathrm{cm}$ & Rod; $34.0 \mathrm{~mm} \mathrm{D,} 50 \mathrm{~mm}$ long \\
\hline 1463 & Iron & 6 to $1000 \mathrm{~K}$ & $10.1 \mu \Omega \cdot \mathrm{cm}$ & Rod; $\quad 6.4 \mathrm{~mm} \mathrm{D,} 50 \mathrm{~mm}$ long \\
\hline 1464 & Iron & 6 to $1000 \mathrm{~K}$ & $10.1 \mu \Omega \cdot \mathrm{cm}$ & Rod; $31.7 \mathrm{~mm} \mathrm{D,} 50 \mathrm{~mm}$ long \\
\hline 1465 & Tungsten & 4 to $3000 \mathrm{~K}$ & $5.4 \mu \Omega \cdot \mathrm{cm}$ & Rod; $\quad 3.2 \mathrm{~mm} \mathrm{D,} 50 \mathrm{~mm}$ long \\
\hline 1466 & Tungsten & 4 to $3000 \mathrm{~K}$ & $5.4 \mu \Omega \cdot \mathrm{cm}$ & Rod; $\quad 6.4 \mathrm{~mm} \mathrm{D}, 50 \mathrm{~mm}$ long \\
\hline 1467 & Tungsten & 4 to $3000 \mathrm{~K}$ & $5.4 \mu \Omega \cdot \mathrm{cm}$ & Rod; $\quad 8.3 \mathrm{~mm} \mathrm{D}, 50 \mathrm{~mm}$ long \\
\hline 1468 & Tungsten & 4 to $3000 \mathrm{~K}$ & $5.4 \mu \Omega \cdot \mathrm{cm}$ & Rod: $10.2 \mathrm{~mm} \mathrm{D,} 50 \mathrm{~mm}$ long \\
\hline
\end{tabular}

\section{Silicon}

These SRM's are intended for use in calibrating four-probe, two-probe, and eddy-current test methods.

\begin{tabular}{|c|c|c|c|}
\hline SRM & Туре & Resistivity & Form \\
\hline 1521 & 111 p-Type Silicon & 0.1 and $10 \Omega \cdot \mathrm{cm}$ & 2 wafers, $51 \mathrm{~mm} \mathrm{D}$ \\
\hline 1522 & 111 n-Type Silicon & 25,75, and $180 \Omega \cdot \mathrm{cm}$ & 3 wafers, $51 \mathrm{~mm} \mathrm{D}$ \\
\hline 1523 & 100 and 111 p-Type Silicon & 0.01 and $1 \Omega \cdot \mathrm{cm}$ & 2 wafers. $51 \mathrm{~mm} \mathrm{D}$ \\
\hline 2526 & 111 p-Type Silicon, Spreading Resistance & 0.001 to $200 \Omega \cdot \mathrm{cm}$ & 15 to 20 slices \\
\hline 2527 & 111 n-Type Silicon, Spreading Resistance & 0.001 to $200 \Omega \cdot \mathrm{cm}$ & 15 to 20 slices \\
\hline 2528 & 100 p-Type Silicon, Spreading Resistance & 0.001 to $200 \Omega \cdot \mathrm{cm}$ & 15 to 20 slices \\
\hline 2529 & 100 n-Type Silicon, Spreading Resistance & 0.001 to $200 \Omega \cdot \mathrm{cm}$ & 15 to 20 slices \\
\hline
\end{tabular}




\section{Glass}

This SRM is useful in evaluating the ASTM C657 method of determining the de volume resistivity of glasses.

\begin{tabular}{|lcrrr|}
\hline SRM & Type & Resistivity & Form \\
\hline 624 & Lead-Silica Glass & $7.6 \mathrm{G} \Omega \cdot \mathrm{cm}$ & $50 \times 50 \times 25 \mathrm{~mm}$ \\
\hline
\end{tabular}

\section{Residual Resistivity Ratio}

This SRM is a set of five aluminum rods that are intended for use in checking four-terminal dc and eddy current decay techniques. The residual resistivity ratio, $\rho(273 \mathrm{~K}) / \rho(4 \mathrm{~K})$, is a sensitive indicator of purity and of the mechanical state of a material.

\begin{tabular}{|lllr|}
\hline SRM & Type & RRR Values & Form \\
\hline 769 & Aluminum & $130,683,1205,2650$, and 11,000 & $6.4 \mathrm{~mm}$ D, 52 mm long \\
\hline
\end{tabular}

\section{Conductivity}

These SRM's are intended for use in the calibration of eddy current conductivity meters and of secondary electrical conductivity standards. Eddy current measurements are used in nondestructive inspection of conducting materials and in the sorting of alloys for composition and heat treatment.

\begin{tabular}{|llll|}
\hline SRM & \multicolumn{1}{|c|}{ Type } & Conductivity & Form \\
\hline 1860 & Aluminum & $60 \%$ IACS & $44 \times 44 \times 9.5 \mathrm{~mm}$ \\
\hline 1861 & Aluminum-Copper Alloy & $47 \%$ IACS & $44 \times 44 \times 9.5 \mathrm{~mm}$ \\
\hline 1862 & Aluminum-Magnesium Alloy & $41 \%$ IACS & $44 \times 44 \times 9.5 \mathrm{~mm}$ \\
\hline 1863 & Aluminum-Copper Alloy & $30 \%$ IACS & $44 \times 44 \times 9.5 \mathrm{~mm}$ \\
\hline
\end{tabular}

\section{Nondestructive Evaluathon}

SRM 1850 is intended for use in checking the performance of liquid dye penetrants and dye penetrant crack detection techniques. It is a test block that contains four synthetic cracks approximately $0.2,0.5,1$, and $2 \mu \mathrm{m}$ wide.

\begin{tabular}{|lcc|}
\hline SRM & Type & Unit \\
\hline 1850 & Penetrant Test Block & 1 each \\
\hline
\end{tabular}




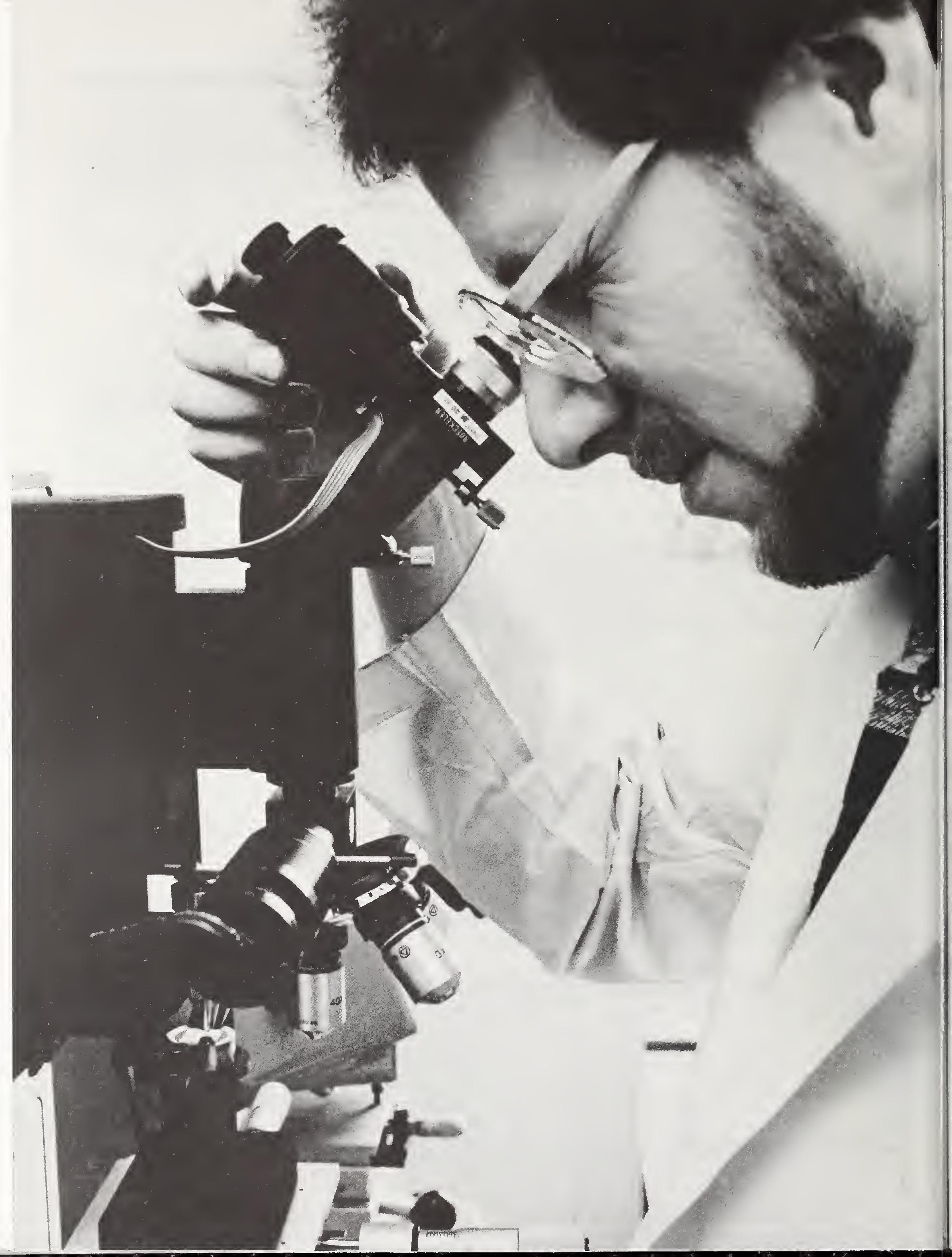




\section{Engineering Type Standards, Research Materials, and Special Reference Materials}

These SRM's are intended to relate measurements used for production or quality control data to a central point of reference. The values certified for these materials are in some cases empirical and do not necessarily relate to the National Measurement System.

\section{Standard Rubbers and Rubber-Compounding Materials}

These SRM's have been prepared to provide the rubber industry with standard materials for rubber compounding. They are useful for the testing of rubber and rubber-compounding materials in connection with quality control of raw materials and for the standardization of rubber testing.

Each material has been statistically evaluated for uniformity by mixing rubber compounds and vulcanizing them in accordance with ASTM Designation D-15 and determining the stress-strain properties of the resulting vulcanizates. Certificates are issued for the rubbers because the properties of different lots are not the same. Replacement lots of rubber-compounding SRM's impart essentially the same characteristics to rubber vulcanizates so that Certificates are not issued for these SRM's.

\section{Standard Rubbers}

\begin{tabular}{|llcc|}
\hline SRM & \multicolumn{1}{c|}{ Type } & Wt/Unit & Pounds \\
\hline $386 \mathrm{~h}$ & Styrene-butadiene 1500 & $34 \mathrm{~kg}$ & 75 \\
\hline $388 \mathrm{~L}$ & Butyl & $34 \mathrm{~kg}$ & 75 \\
\hline 1495 & Butyl (Low Viscosity) & $34 \mathrm{~kg}$ & 75 \\
\hline
\end{tabular}

\begin{tabular}{|c|c|c|c|}
\hline \multicolumn{4}{|c|}{ Rubber Compounding Materials } \\
\hline SRM & Type & Wt/Unit & Pounds \\
\hline $370 \mathrm{e}$ & Zinc Oxide & $8 \mathrm{~kg}$ & 17.6 \\
\hline $371 \mathrm{lg}$ & Sulfur & $6 \mathrm{~kg}$ & 13.2 \\
\hline $372 \mathrm{~h}$ & Stearic Acid & $3.2 \mathrm{~kg}$ & 7.1 \\
\hline $373 \mathrm{f}$ & Benzothiazyl disulfide & $2 \mathrm{~kg}$ & 4.4 \\
\hline $375 \mathrm{~g}$ & Channel Black & $28 \mathrm{~kg}$ & 61.6 \\
\hline $378 \mathrm{~b}$ & Oil Furnace Black & $28 \mathrm{~kg}$ & 61.6 \\
\hline $382 \mathrm{a}$ & Gas Furnace Black & $32 \mathrm{~kg}$ & 70.6 \\
\hline$\overline{383 a}$ & Mercaptobenzothiazale & $3.2 \mathrm{~kg}$ & 7.1 \\
\hline$\overline{384 d}$ & N-tertiary-Butyl-2-benzothiazolesulfenamide & $3.2 \mathrm{~kg}$ & 7.1 \\
\hline
\end{tabular}




\section{Reference Magnetic Tapes}

These SRM's are intended for use in evaluating the performance of magnetic computer tapes and maintaining control over their production. Each SRM is individually calibrated and certified.

\begin{tabular}{|lll|}
\hline SRM & \multicolumn{1}{c}{ Type } & \multicolumn{1}{c|}{ Unit } \\
\hline 1600 & Secondary standard magnetic tape-computer amplitude reference & Cassette \\
\hline 3200 & Secondary standard magnetic tape-computer amplitude reference & Reel/600 feet \\
\hline 3210 & Secondary standard flexible disk cartridge-computer amplitude reference & Flexible disk \\
\hline 3216 & Secondary standard magnetic tape-computer amplitude reference & Cartridge \\
\hline 6250 & Secondary standard high density magnetic tape-computer amplitude reference & Reel/2400 feet \\
\hline
\end{tabular}

\section{Centerline Drawings for Optical Character Recognition-B Characters}

This SRM is an exact copy of the centerline drawings that uniquely define each printed character shape and size used in constant strokewidth Style B Optical Character Recognition (OCR-B) applications in accordance with one or more of the following standards: American National Standard X3.49-1975, Character Set for Optical Character Recognition (OCR-B); Federal Information Processing Standards Publication 32-1974, Optical Character Recognition Character Sets; European Computer Manufacturers Association Standard ECMA-II for the Alphanumeric Character Set OCR-B for Optical Recognition, 3rd Edition, 1975; and Draft International Standard ISO/DIS 1073/II, Alphanumeric Character Sets for Optical Recognition.

This standard contains information on the nominal size, strokewidth, tolerance, and relative position of characters.

\begin{tabular}{llc|}
\hline SRM & Size & OCR.B Characters \\
\hline 1901 & $\mathrm{I}$ & 118 \\
\hline
\end{tabular}
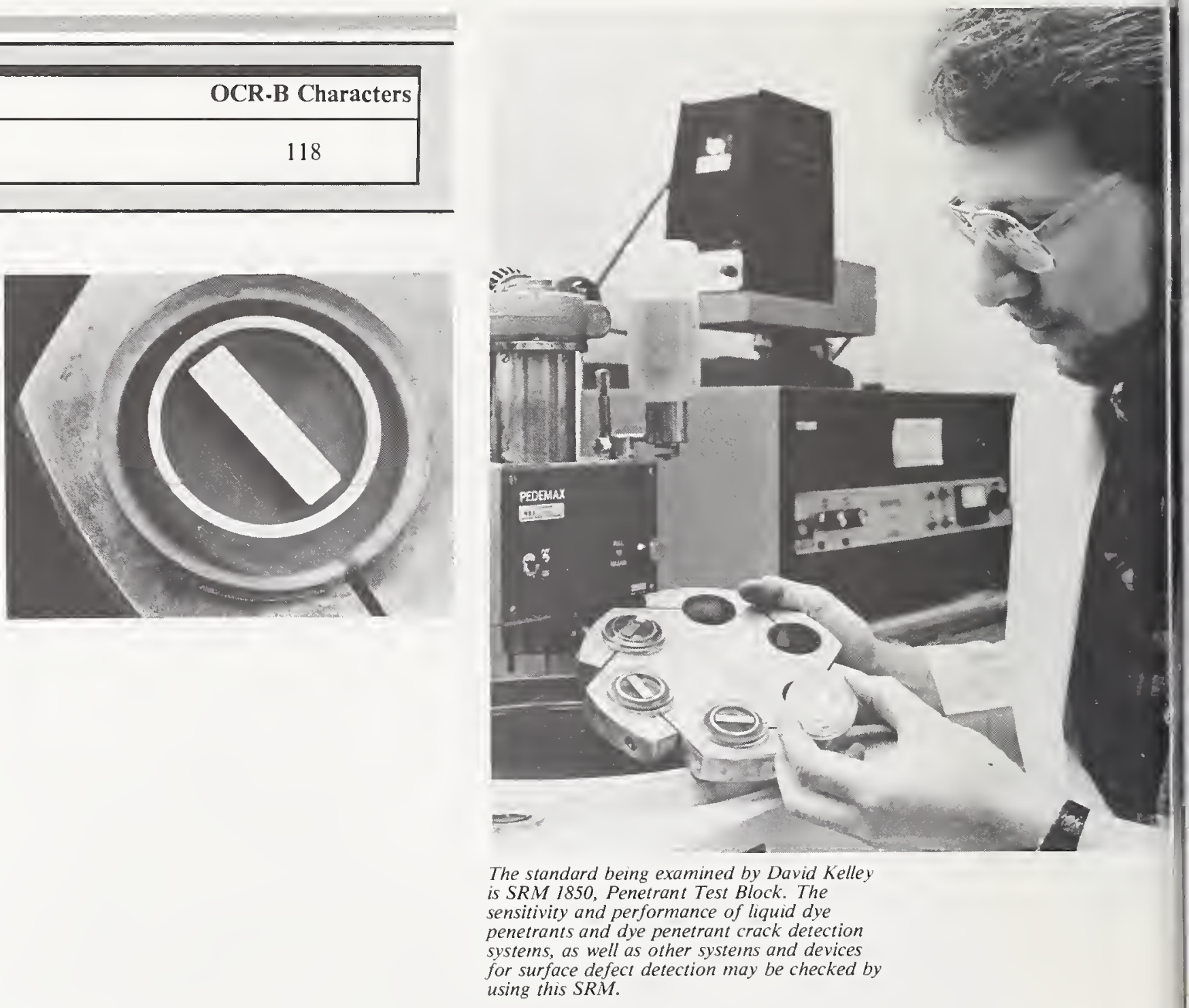


\section{Sizing}

\section{Particle Size}

SRM's $1003 \mathrm{a}$ and 1690 can be used to calibrate various types of particle size measuring instruments including both light and electrical zone flow-through counters. SRM's 1004, 1017a, 1018a, and 1019a are intended for use in calibrating test sieves.

\begin{tabular}{|c|c|c|c|c|}
\hline SRM & Type & Size $(\mu \mathrm{m})$ & Sieve No. & Wt/Unit \\
\hline $1003 \mathrm{a}$ & Glass Spheres & $5-50$ & & IN PREP \\
\hline 1004 & Glass Spheres & $34-120$ & $400-140$ & $63 \mathrm{~g}$ \\
\hline $1017 \mathrm{a}$ & Glass Spheres for calibrating & $100-310$ & $140-50$ & $84 \mathrm{~g}$ \\
\hline $1018 \mathrm{a}$ & Glass Spheres & $225-780$ & $60-25$ & $74 \mathrm{~g}$ \\
\hline $1019 \mathrm{a}$ & Glass Spheres & $650-1900$ & $25-12$ & $200 \mathrm{~g}$ \\
\hline 1690 & Polystyrene Spheres ( $0.5 \%$ wt. concentration in water) & -0.9 & & $5 \mathrm{~mL}$ vial \\
\hline 1691 & Polystyrene Spheres & -0.3 & & IN PREP \\
\hline
\end{tabular}

\section{Cement Turbidimetric and Fineness}

This SRM is available to calibrate the Blaine fineness meter according to the latest issue of Federal Test Method Standard 158, Method 2101 or ASTM Designation C204; to calibrate the Wagner turbidimeter according to ASTM Designation C115; and to determine sieve residue according to ASTM Designation C430. Each set consists of twenty sealed vials, each containing approximately 10 grams of cement.

\begin{tabular}{|lllll}
\hline SRM & Type & Certification & Unit \\
\hline $114 n$ & Portland Cement & Residue on $45 \mu \mathrm{m}$, electroformed sieve wet method & Set of 20 vials \\
\hline & & Surface area (Wagner turbidimeter) & \\
\hline & Surface area (Air-permeability) & \\
\hline
\end{tabular}

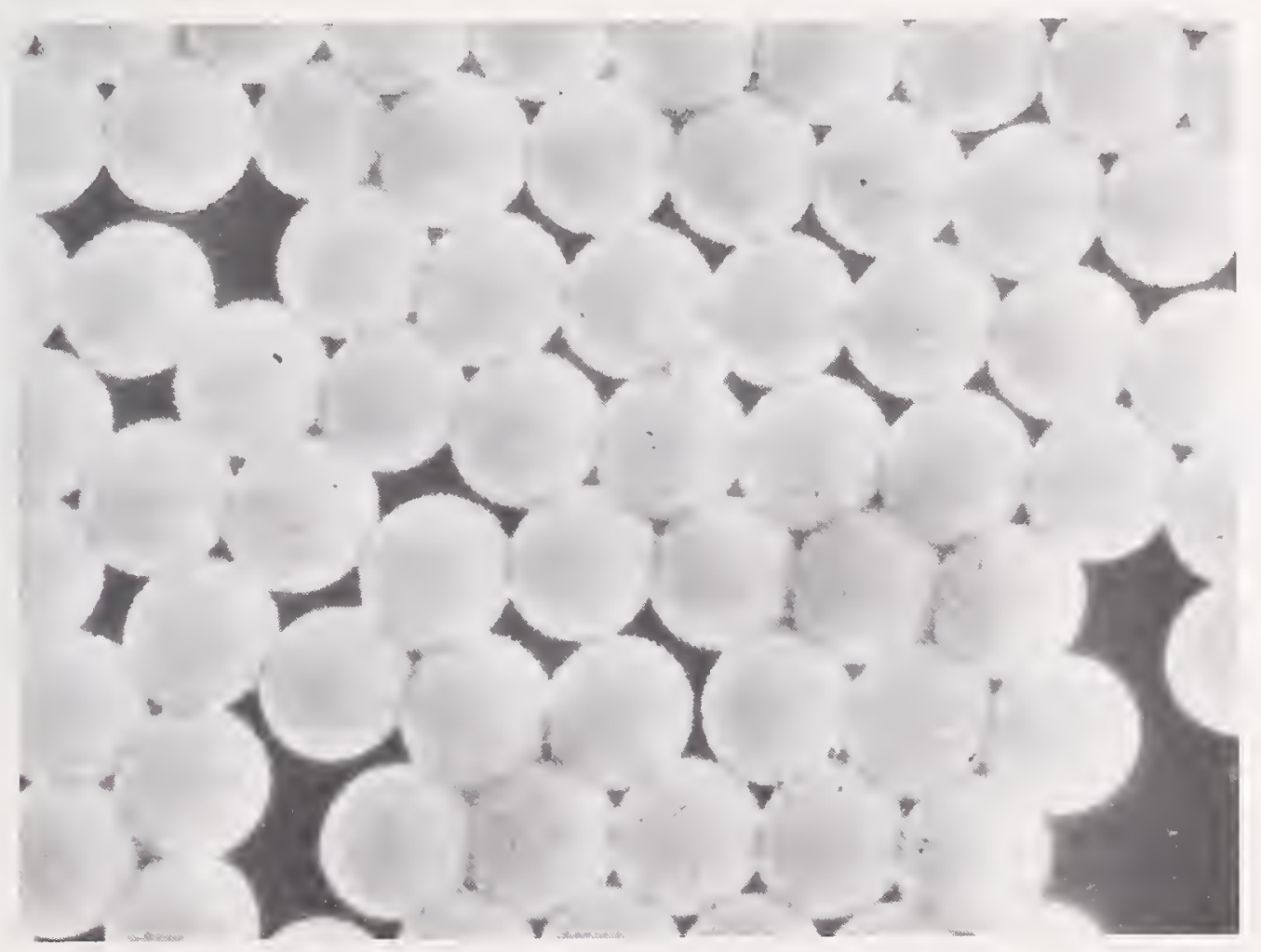

SRM 1690, Nominal One Micrometer Polystyrene Spheres, is a primary particle size reference standard used for calibration of particle size measuring instruments including microscopes. 


\section{Collor}

These SRM's are available to illustrate a characteristic color for each of the ISCC-NBS color-name blocks in NBS Special Publication 440, COLOR: Universal Language and Dictionary of Names. SRM 2106 consists of 251 color chips on 18 constant-hue centroid color charts, and constitutes a supplement to SP 440. SRM 2107 combines SRM 2106 with SP 440 to form a complete color kit. The centroid colors represent a systematic sampling of the whole color solid, each color of which has been carefully measured. Each centroid color has its own specification and can be used as a color standard. (Note: SP 440, may be purchased separately from the Superintendent of Documents, U.S. Government Printing Office, Washington, DC 20402.)

\begin{tabular}{|lll|}
\hline SRM & \multicolumn{1}{|c|}{ Type } & Unit of Issue \\
\hline 2106 & Centroid Color Charts & Set: 18 Charts \\
\hline 2107 & Color Kit & Set: SRM 2106 and SP 440 \\
\hline
\end{tabular}

\section{Light-Sensitive Papers and Plastic Chips}

\section{Light-Sensitive Papers}

Standard light-sensitive paper and booklets of standards faded strips of this paper are available for use in standardizing the dosage of radiant energy when testing textiles for color fastness by exposure in commercial carbon-arc fading lamps. The paper is distributed in units of 100 pieces $25 / 8$ by $31 / 4$ inches. The booklet contains six strips of $11 / 4$ in wide that have been faded by exposure in the NBS master lamp. A copy of NBS Special Publication 260-41, which describes the preparation and use of the materials, is furnished with each booklet.

\begin{tabular}{|lcl|}
\hline SRM & Type & Unit of Issue \\
\hline $700 \mathrm{~d}$ & Light-sensitive paper & Pkg. of 100 pieces- $-25 / 8$ in $\times 31 / 4$ in \\
\hline $701 \mathrm{~d}$ & Standard faded strips & Booklet -6 strips $11 / 4$ in wide \\
\hline
\end{tabular}

\section{Light-Sensitive Plastic Chips}

Standard light-sensitive plastic chips are available for use in calibration and standardization of artificial weathering and fading apparatus. These chips are distributed in units of five plates 2 in by $41 / 8$ in, and have been standardized by the measurement of the change of transmittance as a function of exposure (in standard fading hours) to the NBS master lamps.

\begin{tabular}{|lcc|}
\hline SRM & Type & Unit of Issue \\
\hline 703 & Light-sensitive plastic chips & Package of 5 chips 0.060 in thick \\
\hline
\end{tabular}




\section{X-ray and Photographic}

SRM 1001 is a calibrated $\mathrm{x}$-ray film step tablet of 17 steps that cover the optical density range from 0 to 4

SRM 1008 is a calibrated photographic step tablet of 21 steps that cover the optical density range from 0 to 4 .

Both step tablets are individually calibrated and certified for diffuse transmission density in

conformance with conditions specified for American National Standard Diffuse Visual Transmission Density, $\mathrm{D}_{\mathrm{t}}\left(90 ; 3000 \mathrm{~K}: \leqslant 10^{\circ} ; \mathrm{V}\right)$, in "ANSI PH 2.19-1976, American National Standard for Diffuse and Doubly

Diffuse Transmission Measurements (Transmission Density)."

SRM 1010a, Microcopy Resolution Test Charts, is used to test the resolving power of cameras or of whole microcopying systems. SRM 1010a consists of five charts printed photographically on paper, and have 26 high-contrast five-line patterns ranging in spatial frequency from one cycle per millimeter to 18 cycles per millimeter. Instructions for the use of the charts are supplied with each order.

SRM 2061 is a calibrated reflection step tablet having 12 steps that cover the optical density range from 0 to 2 on gray scale paper from white to black. It is intended primarily for use in photographic applications requiring color balance or separation.

SRM 2061 is calibrated using methods that conform to conditions specified for American National Standard Annular $45^{\circ} ; 0^{\circ}$ Absolute Visual Reflection Density, Type $3000 \mathrm{~K}, \mathrm{D}_{\mathrm{R}_{\mathrm{a}}}\left(\mathrm{g}_{\mathrm{a}} ; 3000 \mathrm{~K}: \leqslant 5^{\circ} ; \mathrm{V}\right)$ in ANSI PH 2.17-1977.

\begin{tabular}{|llc|}
\hline \multicolumn{1}{|c|}{ Type } & Unit \\
\hline 1001 & X-ray Film Step Tablet $(0-4)$ & 1 tablet, 17 steps \\
\hline 1008 & Photographic Step Tablet $(0-4)$ & 1 tablet, 21 steps \\
\hline $1010 a$ & Microcopy Resolution Test Chart & Set of 5 charts \\
\hline 2061 & Reflection Step Tablet & 1 tablet, 12 Steps \\
\hline
\end{tabular}

For further information regarding calibration services, call 301-921-2805.

\section{Surface Flammability}

SRM 1002c, Hardboard Sheet, is issued for checking the operation of radiant panel test equipment in accordance with the procedures outlined in ASTM Standard E162-78.

\begin{tabular}{|lccc|}
\hline SRM & Type & Certification & Unit of Issue \\
\hline $1002 \mathrm{c}$ & Hardboard Sheet & Flame Spread Index, $\mathrm{I}=153$ & Set of $4: 6 \times 18 \times 1 / 4$ inch \\
& Heat Evolution Factor, $\mathrm{Q}=36.5$ & \\
\hline
\end{tabular}

\section{Smoke Density Chamber}

These SRM's are certified for maximum specific optical density and are issued for performing operational checks of smoke density chambers.

\begin{tabular}{|lllll|}
\hline SRM & Type & Maximum Specific Optical Density & Unit of Issue \\
\hline $1006 \mathrm{~b}$ & Non-flaming Exposure Condition $(\alpha$-cellulose) & Dm $($ corr. $)=183 \pm 25$ & 3 sheets \\
\hline $1007 \mathrm{a}$ & Flaming Exposure Condition (plastic) & Dm $($ corr. $)=17850(\mathrm{t})-132$ & 3 sheets \\
\hline
\end{tabular}




\section{Tape Adhesion Testing}

This material is intended as a uniform source of linerboard for use under ASTM Designation D2860, Procedure A: Adhesion of Pressure Sensitive Tape to Fiberboard at 90 Degree Angle and Constant Stress.

\begin{tabular}{|ccc}
\hline SRM & Type & Unit \\
\hline 1810 & Linerboard for Tape Adhesion Testing & Package of 50 sheets
\end{tabular}

\section{Research Materialls}

Research Materials (RM's) are distinct from the Standard Reference Materials (SRM's) issued by NBS. The distinction between RM's and SRM's is in the information supplied with them and the purpose for which they are used. Unlike SRM's, RM's are not issued with Certificates of Analysis; rather they are accompanied by a "Report of Investigation," the sole authority of which is the author of the report. RM's are intended primarily to promote scientific or technical research on a particular material. One of the principal reasons for issuing an RM is to provide a homogeneous material so that an investigator in one laboratory can be assured that the material he has is the same as that being investigated in a different laboratory. The following materials are available:

\section{RM 1C-Ultra-Purity Aluminum Single Crystal Cubes}

These cubes are intended for use in studies of a variety of solid state phenomena for which both extreme purity and knowledge of crystallographic orientation are required; e.g., in studies of electron spin resonance, De Haas-Van Alphen effect, cyclotron resonance, and in a variety of studies relating to the Fermi surface and the transport properties of aluminum. Unit of issue: $1 \mathrm{~cm}$ on a side.

\section{RM 1 R-Ultra-Purity Aluminum Polycrystalline Rods}

These rods are intended for use in research on the mechanical and physical properties of extremely pure aluminum; e.g., in the determination of resistivity as a function of strain at cryogenic temperatures to facilitate the design of cryogenic magnets or superconductor stabilizing elements. Unit of issue: $4.2 \mathrm{~mm}$ in diameter and $25.4 \mathrm{~mm}$ long.

\section{RM 5-Copper Heat Capacity Test Specimen}

This material is intended for use in the comparison of heat capacity results from different laboratories and as a test specimen for heat capacity measurement below $25 \mathrm{~K}$. It may also be useful at higher temperatures. RM

SRM 3200, Secondary Standard Magnetic Tape, Computer Amplitude Reference, is used for calibrating the output signal amplitude from computer lape recordings and reproducing systenis. Jaines Park notates readings during the production of this SRM.

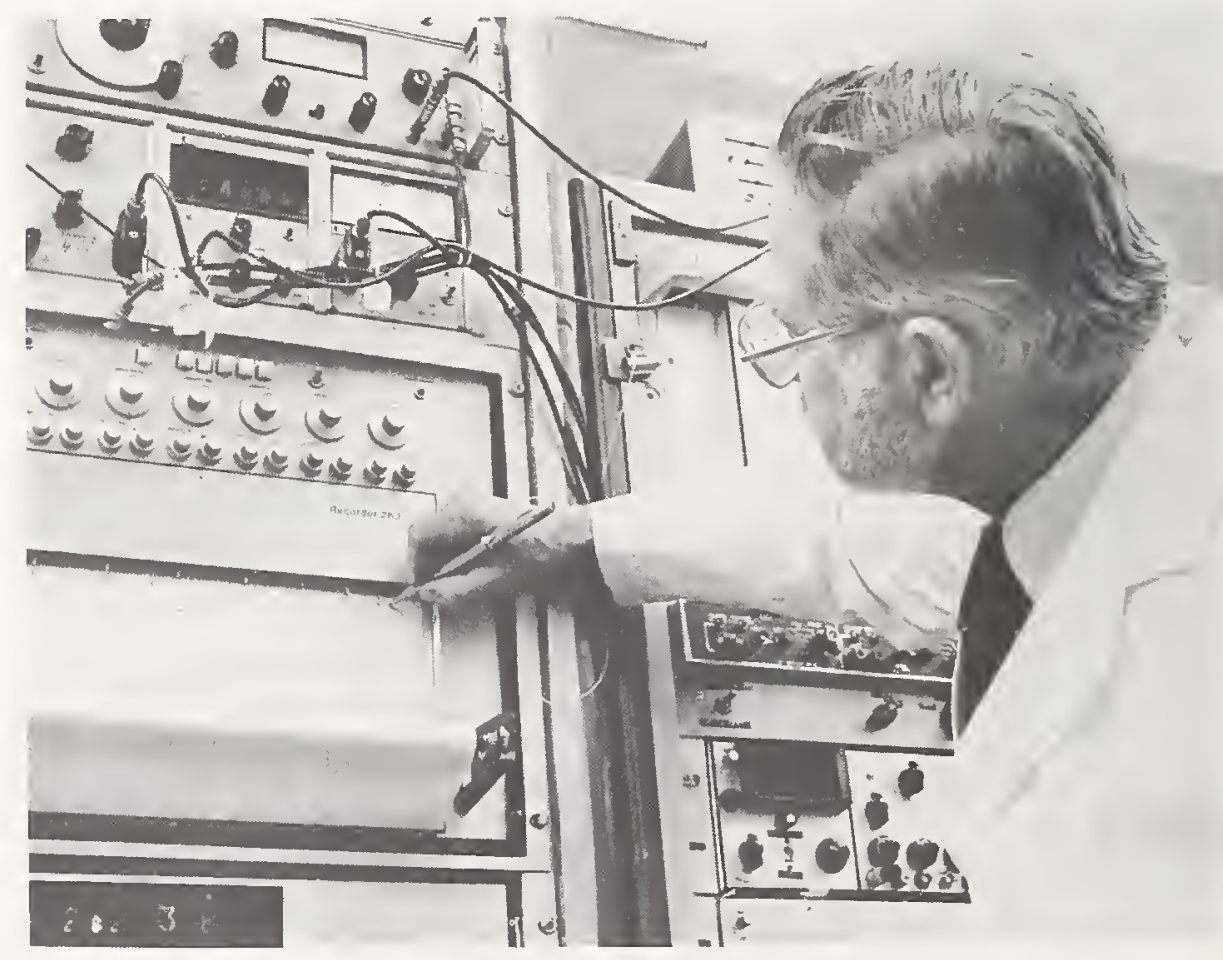


5 is available as a rod of high purity polycrystalline copper. Unit of issue: $19 \mathrm{~mm}$ in diameter and $120 \mathrm{~mm}$ long.

\section{RM 31-Glass Fibers for Microanalysis}

This monogeneous vitreous solid contains known, low-concentration additions of several elements which were developed for electron probe microanalysis (EMPA) and secondary ion mass spectrometry (SIMS). This RM contains ten compositions of various oxides. Unit of issue: set of 10 .

\section{RM 45b-Homogeneous River Sediment}

This specimen is a freeze-dried river sediment intended for use in testing radiochemical procedures for the assay of radioactivity in sediments and soils. Unit of issue: $100 \mathrm{~g}$.

\section{RM 50-Albacore Tuna}

This material is intended to be used in the measurement of elements present at trace concentration. It has been issued as a lyophilized (freeze-dried) marine biological tissue sample in an attempt to satisfy many of the analytical requirements for a base-line marine reference material. The Report of Investigation provides uncertified informational data on mercury, selenium, zinc, arsenic, lead, and a number of other elements of interest to marine scientists. Unit of issue: two $35 \mathrm{~g}$ cans.
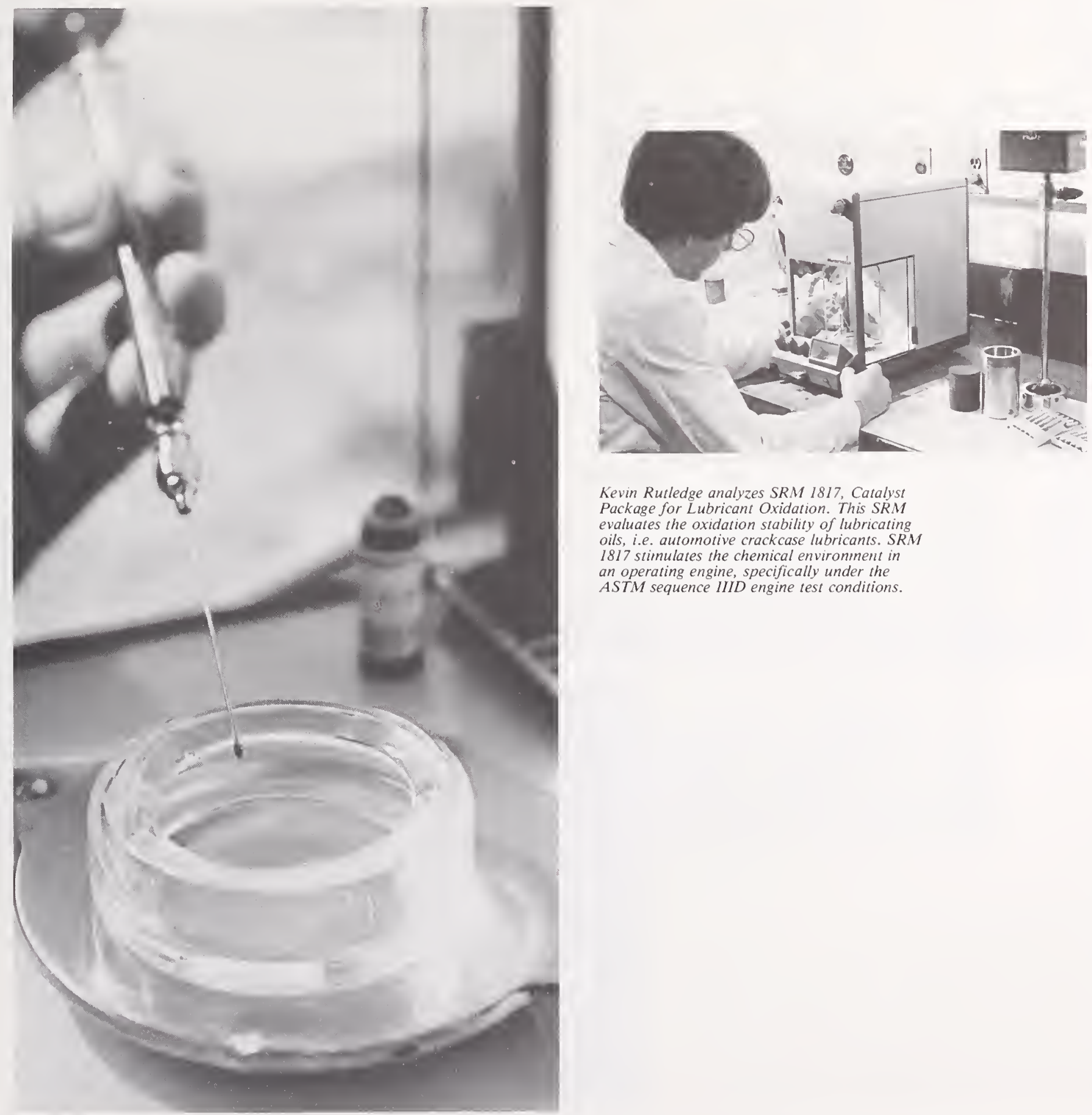

Kevin Rutledge analyzes SRM 1817, Catalyst Package for Lubricant Oxidation. This SRM evaluates the oxidation stability of lubricating oils, i.e. automotive crackcase lubricants. SRM 1817 stimulates the chemical environment in an operating engine, specifically under the ASTM sequence IIID engine test conditions. 


\section{Special Reference Materials}

Special Reference Materials (GM's) are distributed by NBS to meet industry needs. These materials have been standardized either by some Government agency other than NBS, or by some standards-making body such as the American Society for Testing and Materials (ASTM), the American National Standards Institute (ANSI), and the Organization for International Standardization (ISO). For this class of materials, NBS acts only as a distribution point and does not participate in the standardization of these materials.

\section{Differential Thermal Analysis}

GM's 754, 757, 758, 759, 760, and 761 have been issued by NBS in cooperation with the International Confederation of Thermal Analysis as standards for calibrating differential thermal analysis, differential scanning calorimetry, and thermogravimetry equipment under operating conditions.

\begin{tabular}{|c|c|c|c|c|}
\hline \multirow{2}{*}{$\begin{array}{l}\text { GM } \\
754 \\
\end{array}$} & \multicolumn{2}{|c|}{ Material } & \multirow{2}{*}{$\frac{\text { Peak Temp. }\left({ }^{\circ} \mathrm{C}\right)}{-105^{\circ} \mathrm{C}}$} & \multirow{2}{*}{$\begin{array}{l}\text { Uṇit } \\
10 \mathrm{~g}\end{array}$} \\
\hline & Polystyrene & (glass transition) & & \\
\hline \multirow[t]{6}{*}{757} & 1,2-Dichloroethane & (melting point) & $-32^{\circ} \mathrm{C}$ & $4 \mathrm{~mL}$ \\
\hline & Clycohexane & & & \\
\hline & & (transition point) & $\sim-83^{\circ} \mathrm{C}$ & $4 \mathrm{~mL}$ \\
\hline & & (melting point) & $+7{ }^{\circ} \mathrm{C}$ & \\
\hline & Phenyl Ether & (melting point) & $-30^{\circ} \mathrm{C}$ & $4 \mathrm{~mL}$ \\
\hline & o-Terphenyl & (melting point) & $\sim 58^{\circ} \mathrm{C}$ & $5 \mathrm{~g}$ \\
\hline \multirow[t]{5}{*}{758} & Potassium Nitrate & (transition point) & $-128^{\circ} \mathrm{C}$ & $10 \mathrm{~g}$ \\
\hline & Indium & (melting point) & $-157^{\circ} \mathrm{C}$ & $3 \mathrm{~g}$ \\
\hline & Tin & (melting point) & $-232^{\circ} \mathrm{C}$ & $3 \mathrm{~g}$ \\
\hline & Potassium Perchlorate & (transition point) & $-300^{\circ} \mathrm{C}$ & $10 \mathrm{~g}$ \\
\hline & Silver Sulfate & (transition point) & $-430^{\circ} \mathrm{C}$ & $3 \mathrm{~g}$ \\
\hline \multirow[t]{5}{*}{759} & Potassium Perchlorate & (transition point) & $-300{ }^{\circ} \mathrm{C}$ & $10 \mathrm{~g}$ \\
\hline & Silver Sulfate & (transition point) & $-430^{\circ} \mathrm{C}$ & $3 \mathrm{~g}$ \\
\hline & Quartz & (transition point) & $-573^{\circ} \mathrm{C}$ & $3 \mathrm{~g}$ \\
\hline & Potassium Sulfate & (transition point) & $-583^{\circ} \mathrm{C}$ & $10 \mathrm{~g}$ \\
\hline & Potassium Chromate & (transition point) & $-665^{\circ} \mathrm{C}$ & $10 \mathrm{~g}$ \\
\hline \multirow[t]{5}{*}{760} & Quartz & (transition point) & $-573{ }^{\circ} \mathrm{C}$ & $3 \mathrm{~g}$ \\
\hline & Potassium Sulfate & (transition point) & $-583^{\circ} \mathrm{C}$ & $10 \mathrm{~g}$ \\
\hline & Potassium Chromate & (transition point) & $-665^{\circ} \mathrm{C}$ & $10 \mathrm{~g}$ \\
\hline & Barium Carbonate & (transition point) & $-810^{\circ} \mathrm{C}$ & $10 \mathrm{~g}$ \\
\hline & Strontium Carbonate & (transition point) & $-925^{\circ} \mathrm{C}$ & $10 \mathrm{~g}$ \\
\hline \multirow[t]{5}{*}{761} & Permanorm 3 & (magnetic transition) & $-259^{\circ} \mathrm{C}$ & $1 \mathrm{~g}$ \\
\hline & Nickel & (magnetic transition) & $-353^{\circ} \mathrm{C}$ & $1 \mathrm{~g}$ \\
\hline & Mumetal & (magnetic transition) & $-381^{\circ} \mathrm{C}$ & $1 \mathrm{~g}$ \\
\hline & Permanorm 5 & (magnetic transition) & $-454^{\circ} \mathrm{C}$ & $1 \mathrm{~g}$ \\
\hline & Trafoperm & (magnetic transition) & $-750^{\circ} \mathrm{C}$ & $1 \mathrm{~g}$ \\
\hline
\end{tabular}




\section{Melting Point}

GM 8000 is issued by NBS in cooperation with the Office of Reference Materials at the National Physical Laboratory (NPL) in Teddington, England. This set of ten highly purified substances is intended for use in the calibration of thermometry used in the determining the melting points of samples in glass capillary tubes. Both the meniscus point and the liquefaction point for each substance are certified by NPL.

\begin{tabular}{|c|c|c|c|}
\hline GM & Type & Melting Point & Amount \\
\hline \multirow[t]{10}{*}{8000} & 4-Nitrotoluene & $-52{ }^{\circ} \mathrm{C}$ & $1 \mathrm{~g}$ \\
\hline & Naphthalene & -80 & $1 \mathrm{~g}$ \\
\hline & Benzil & -95 & $1 \mathrm{~g}$ \\
\hline & Acetanilide & -114 & $1 \mathrm{~g}$ \\
\hline & Benzoic Acid & -122 & $1 \mathrm{~g}$ \\
\hline & Diphenylacetic Acid & -147 & $1 \mathrm{~g}$ \\
\hline & Anisic Acid & -183 & $1 \mathrm{~g}$ \\
\hline & 2-Chloroanthraquinone & -210 & $1 \mathrm{~g}$ \\
\hline & Carbazole & -246 & $1 \mathrm{~g}$ \\
\hline & Anthraquinone & -285 & $1 \mathrm{~g}$ \\
\hline
\end{tabular}

\section{Specific Surface Area of Powders}

GM's 8001 through 8008 are issued by NBS in cooperation with the Office of Reference Materials at the National Physical Laboratory (NPL) in Teddington, England. These materials are intended for use in the calibration and checking of instruments for determining the specific surface area of powders by BET.

\begin{tabular}{|llcr|}
\hline GM & \multicolumn{1}{c}{ Type } & Surface Area & Amount \\
\hline 8001 & Graphitized Carbon Black & $11 \mathrm{~m}^{2} \cdot \mathrm{g}^{-1}$ & $10 \mathrm{~g}$ \\
\hline 8002 & Graphitized Carbon Black & 71 & $10 \mathrm{~g}$ \\
\hline 8003 & Non-Porous Silica & 166 & $10 \mathrm{~g}$ \\
\hline 8004 & Meso-Porous Silica & 286 & $10 \mathrm{~g}$ \\
\hline 8005 & Alpha Alumina & 2.1 & $50 \mathrm{~g}$ \\
\hline & & & $50 \mathrm{~g}$ \\
\hline 8006 & Alpha Alumina & 0.3 & $50 \mathrm{~g}$ \\
\hline 8007 & Alpha Alumina & 0.1 & $50 \mathrm{~g}$ \\
\hline 8008 & Alpha Alumina & 0.8 & (1) \\
\hline
\end{tabular}

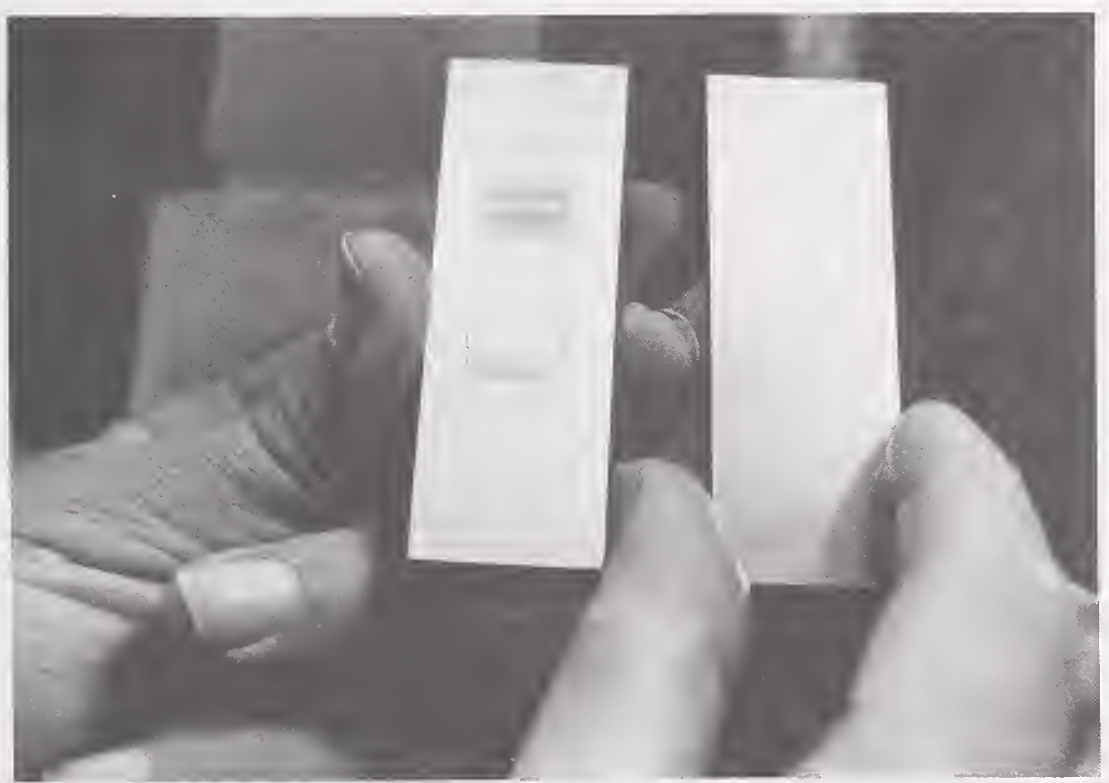

Tool Steel Abrasive Wear Standard, SRM

1857, is intended for use with the dry sand.

rubber wheel abrasion wear method as

described in ASTM Standard Practice G65-81,

Procedure $A$. 


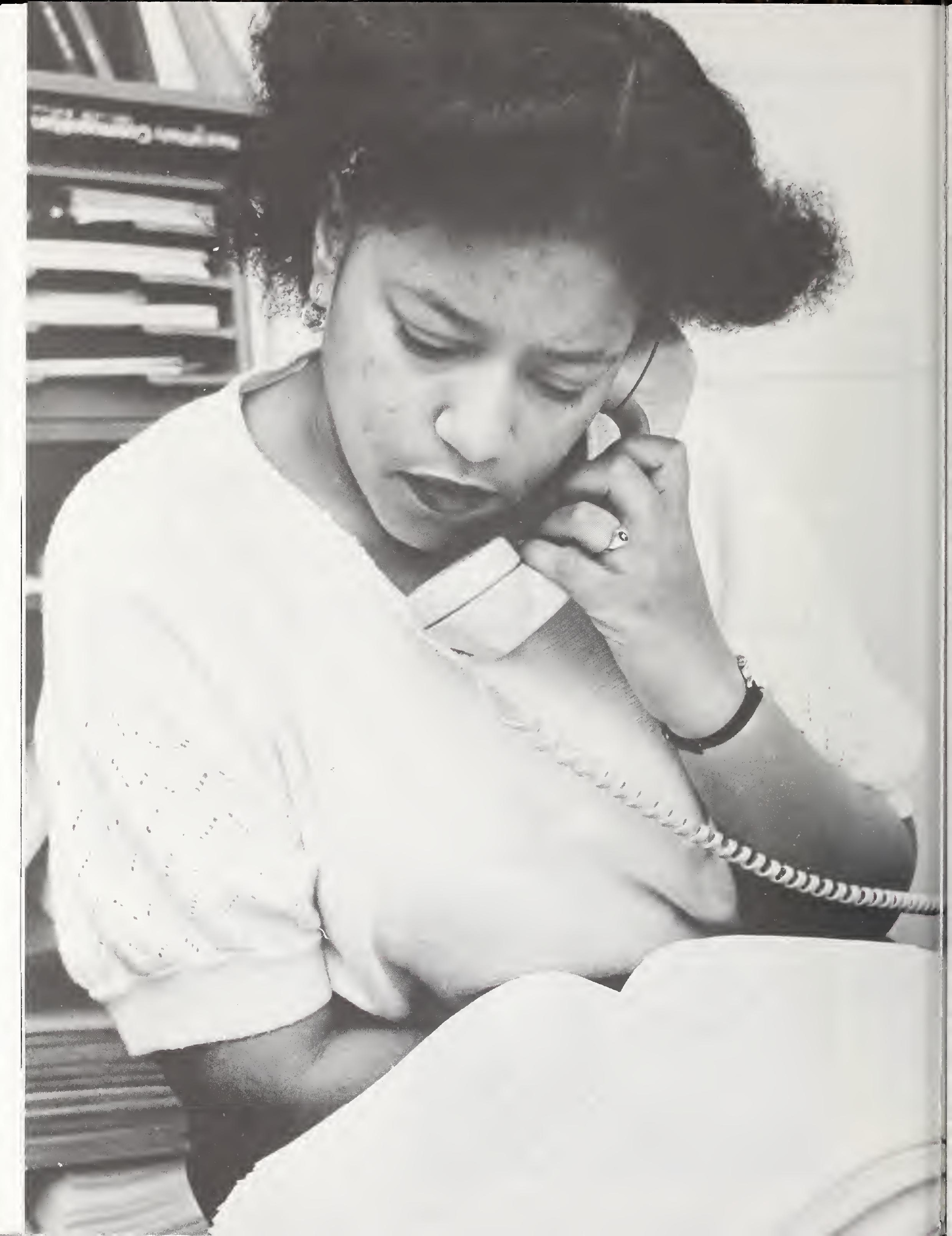




\section{Additional Information}

\section{Other Services of the $\mathbb{N a t i o n a l ~ B u r e a u ~ o f ~ S t a n d a r d s ~}$}

The following is a list of some of the services offered by NBS that may be of interest to SRM users. For general information see the entry on Technical Information and Publications.

\section{Calibration and Related Measurement Services}

The measurement services of the National Bureau of Standards include the calibration of standards, test of instruments, and certain interlaboratory testing programs. These services are listed in NBS Special

Publication 250, Calibration and Related Measurement Services of the National Bureau of Standards.

[Available from the Superintendent of Documents, U.S. Government Printing Office, Washington, DC 20402

(1982 edition) for $\$ 6.00]$.

These services are performed at the National Bureau of Standards Washington laboratories

(Gaithersburg, MD) or the National Bureau of Standards laboratories in Boulder, CO.

An abbreviated list of the services offered under this program is given below. For information concerning services not listed below or in NBS Special Publication 250 contact:

Office of Measurement Services

National Bureau of Standards

Washington, DC 20234

Telephone: (301) 921-2805

\section{Washington Services}

\begin{tabular}{|lr}
\hline AC-DC Wattmeters; AC Resistors; Calibrators and Voltmeters (up to 10 Hz) & $301-921-2727$ \\
\hline Acoustic Measurements & $301-921-3607$ \\
\hline Aerodynamics & $301-921-3684$ \\
\hline Angular & $301-921-2216$ \\
\hline Complex Standards of Length and Diameter & $301-921-2216$ \\
\hline Data Converters & $301-921-2727$ \\
\hline Dosimetry for High-dose Applications & $301-921-2201$ \\
\hline Dosimetry for High-energy Electron Beams & $301-921-2361$ \\
\hline Electrical Instruments (AC-DC) & $301-921-2216$ \\
\hline Flatness, Straightness, and Roundness & $301-921-2216$ \\
\hline & $301-921-3681$ \\
\hline Fluid Quantity and Flow Rate Meters & $301-921-3884$ \\
\hline Force Transducers and Force Measurement Systems & $301-921-2361$ \\
\hline Gamma-ray and Beta-particle Sources & $301-921-3748$ \\
\hline Humidity Measurements & $301-921-2791$ \\
\hline Image Optics and Photography &
\end{tabular}




\section{Washington Services (Continued)}

\begin{tabular}{|c|c|}
\hline Impedance Measurements & $301-921-2715$ \\
\hline Laboratory Thermometers & $301-921-2087$ \\
\hline Length & $301-921-2216$ \\
\hline Length and Diameter Dimensional Metrology & $301-921-2216$ \\
\hline Mass & $301-921-2461$ \\
\hline Near and Vacuum Ultraviolet Radiometric Standards & $301.921-2356$ \\
\hline Photometric Standards & $301-921-3613$ \\
\hline Neutron Sources and Dosimetry Standardization & $301-921-2767$ \\
\hline Precision Apparatus & $301-921-2715$ \\
\hline Pressure and Vacuum Measurements & $301-921-2121$ \\
\hline Radiation Thermometry & $301-921-3613$ \\
\hline Radioactivity & $301-921-2665$ \\
\hline Radiometric Standards & $301-921-3613$ \\
\hline Resistance Measurements & $301-921-2715$ \\
\hline Resistance Thermometers & $301-921-2757$ \\
\hline Spectrophotometric Standards & $301-921-2453$ \\
\hline Surface Texture & $301-921-2159$ \\
\hline Thermocouples and Thermocouple Materials & $301-921-2069$ \\
\hline Ultrasonic Measurements & $301-921-3646$ \\
\hline Vibration Measurements & $301-921-3607$ \\
\hline Voltage, Current, and Power Measurement Devices & $301-921-3121$ \\
\hline Voltage Measurements & $301.921-2715$ \\
\hline Volume and Density & $301-921-2681$ \\
\hline X-ray and Gamma-ray Measuring Instr & 301.921 .2361 \\
\hline
\end{tabular}

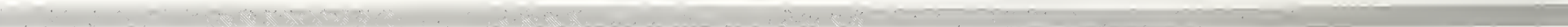

\section{Boulder Services}

All measurement seryices available in Boulder should be directed to:

Measurement Services Clerk

National Bureau of Standards

Boulder, CO 80303

Telephone: (303) 497-3753

Cryogenics

Electromagnetic Quantities

Attenuation

Fields

Impedance or Admittance

Laser Parameters

Noise Temperature (Effective)

Phase Shift

Power Measurements

Voltage

Time and Frequency

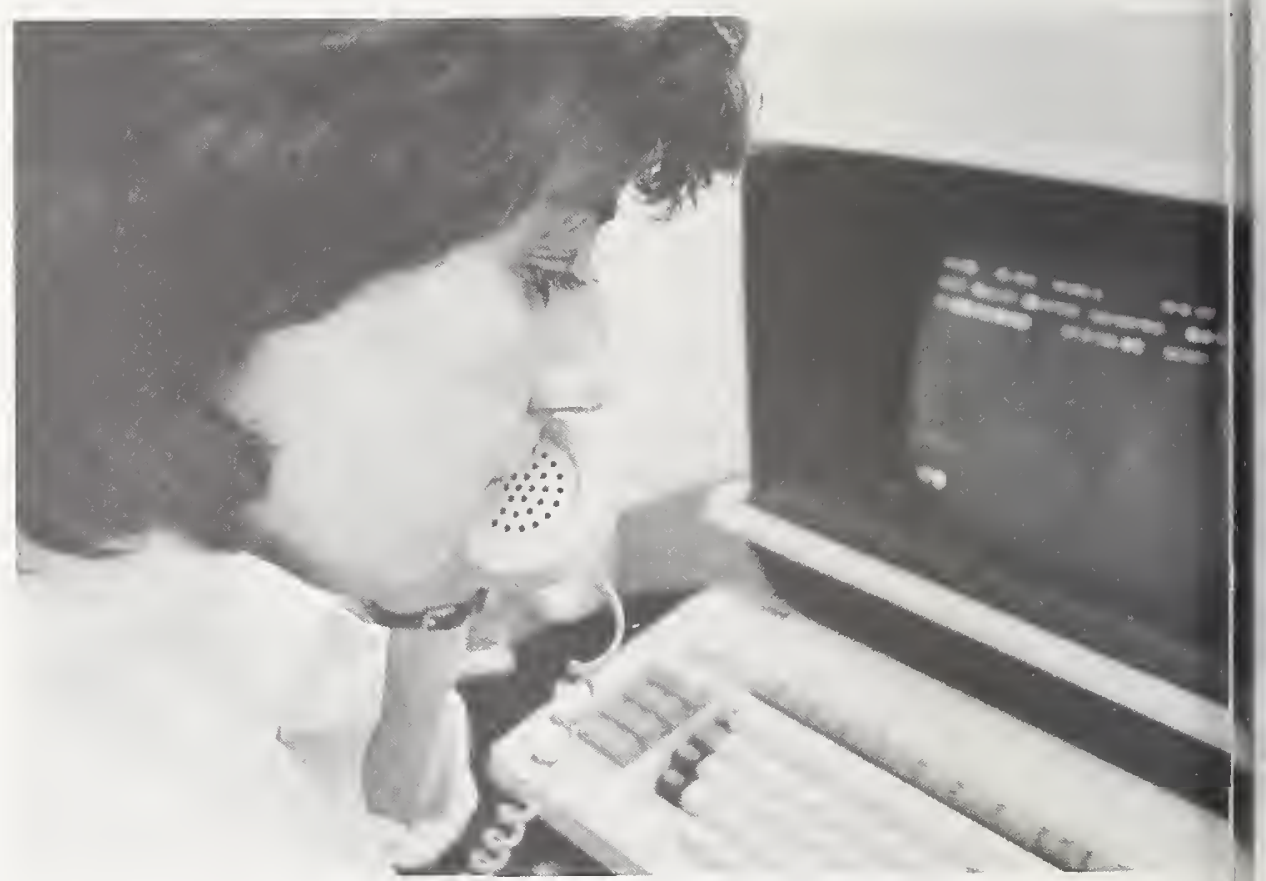

Computerized data allows Patricia Brietic to provide information on the availability of an SRM to a telephone customer. 


\section{Office of Weights and Measures}

The NBS Office of Weights and Measures (OWM) operates a Prototype Examination Program which provides for an evaluation of (1) prototype weighing and measuring devices to determine compliance with the requirements of NBS Handbook 44, "Specifications, Tolerances, and Other Technical Requirements for Commercial Weighing ànd Measuring Devices," (2) standards to determine compliance with the requirements of NBS Handbook 105-1, 105-2, 105-3 "Specifications and Tolerances for Reference Standard and Field Standard Weights and Measures." This program may be used by manufacturers and weights and measures officials in determining the acceptability of devices for commercial use or the suitability of reference and field standards. For information on programs of NBS and the States, contact:

Office of Weights and Measures

A 359, Physics Building

National Bureau of Standards

Washington, DC 20234

(301) $921-2401$

\section{Proficiency Sample Programs}

General information about the Proficiency Sample Programs may be obtained from:

Proficiency Sample Program

National Bureau of Standards

Washington, DC 20234

(301) $921-3481$

Information is available for the following programs:

Proficiency Sample Programs for Hydraulic Cements and Portland Cement Concrete

Proficiency Sample Programs for Soils, Aggregates, and Bituminous Materials

Inspection of Cement and Concrete Testing Laboratories

Inspection of Soils and Bituminous Testing Laboratories

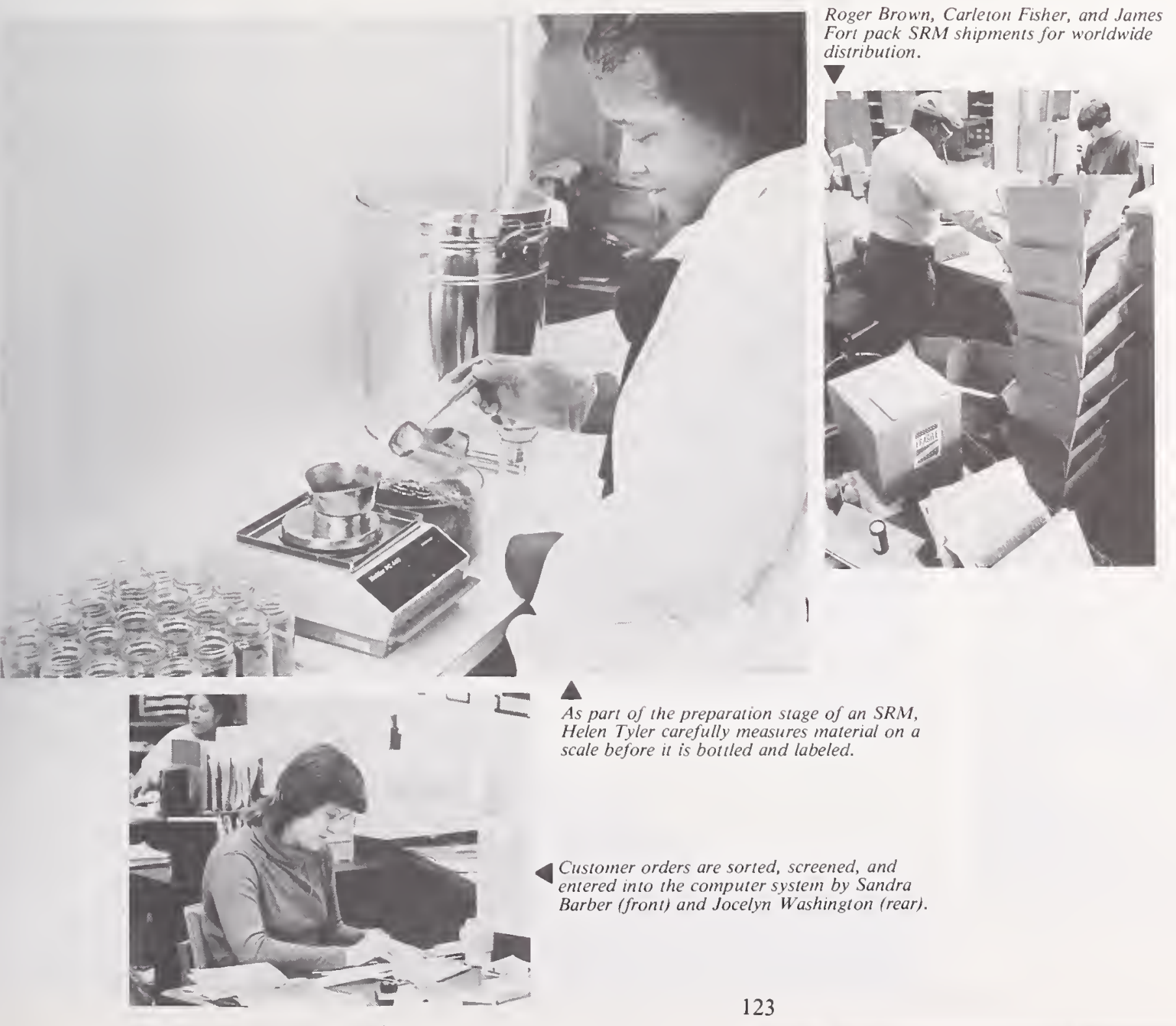




\section{Structural Engineering-High Capacity Testing Machine}

The research and testing facilities for structural engineering include a 53-MN (12 million-lbf) capacity universal testing machine believed to be the largest in the world. A significant addition to the nation's facilities for research and testing in the field of large structures, this unique machine is available to do work for the entire technological community upon consideration of requests on a case-by-case basis. This hydraulically operated machine is a vertical, four screw type with the main fixed platen flush wit the floor. It is capable of applying $53-\mathrm{MN}\left(12 \times 10^{6} \mathrm{lbf}\right)$ in compression to test specimens up to $17 \mathrm{~m}(58 \mathrm{ft})$ in height and $27 \mathrm{MN}\left(6 \times 10^{6} \mathrm{lbf}\right)$ in tension to specimens up to $16 \mathrm{~m}(53 \mathrm{ft})$ in length. To extend the versatility of the machines, the reinforced concrete foundation incorporates a floor tie-down system which can accommodate test specimens for transverse loading up to $27 \mathrm{~m}$ (90 ft) in length. Calibration of all load ranges indicates that they exhibit error generally no greater than 0.5 percent of the applied load. For more information please contact:

Structural Engineering Program

B168, Building Research

National Bureau of Standards

Washington, DC 20234

(301) $921-3471$

\section{Accreditation of Testing Laboratories}

General information about the National Voluntary Laboratory Accreditation Program (NVLAP) or application packages may be obtained from:

Laboratory Accreditation Manager

Room B141, Technology Building

National Bureau of Standards

Washington, DC 20234

(301) $921-3431$

Information is available for the following specific testing areas:

Program for Thermal Insulation Materials

Program for Freshly Mixed Field Concrete

Program for Carpet

Program for Solid Fuel Room Heaters

Program for Personnel Dosimeters Processors

Program for Electromagnetic Calibration Services

Program for Window and Door Products

Acoustical Testing Services

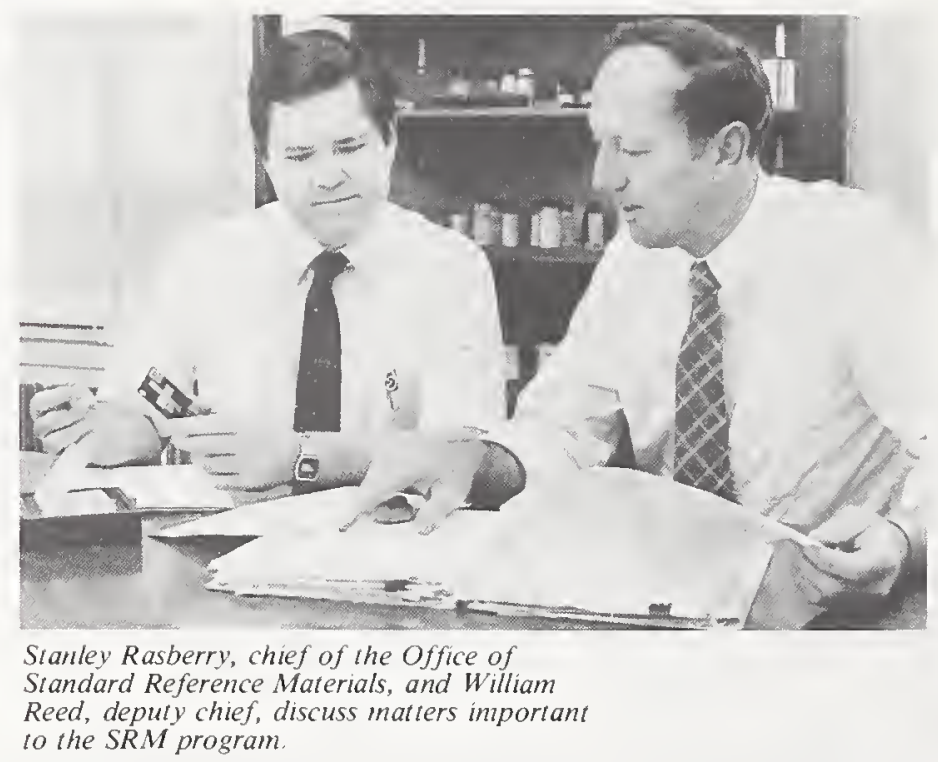

Lee Kieffer, project manager, Thomas Gills, production manager, and William Reed,

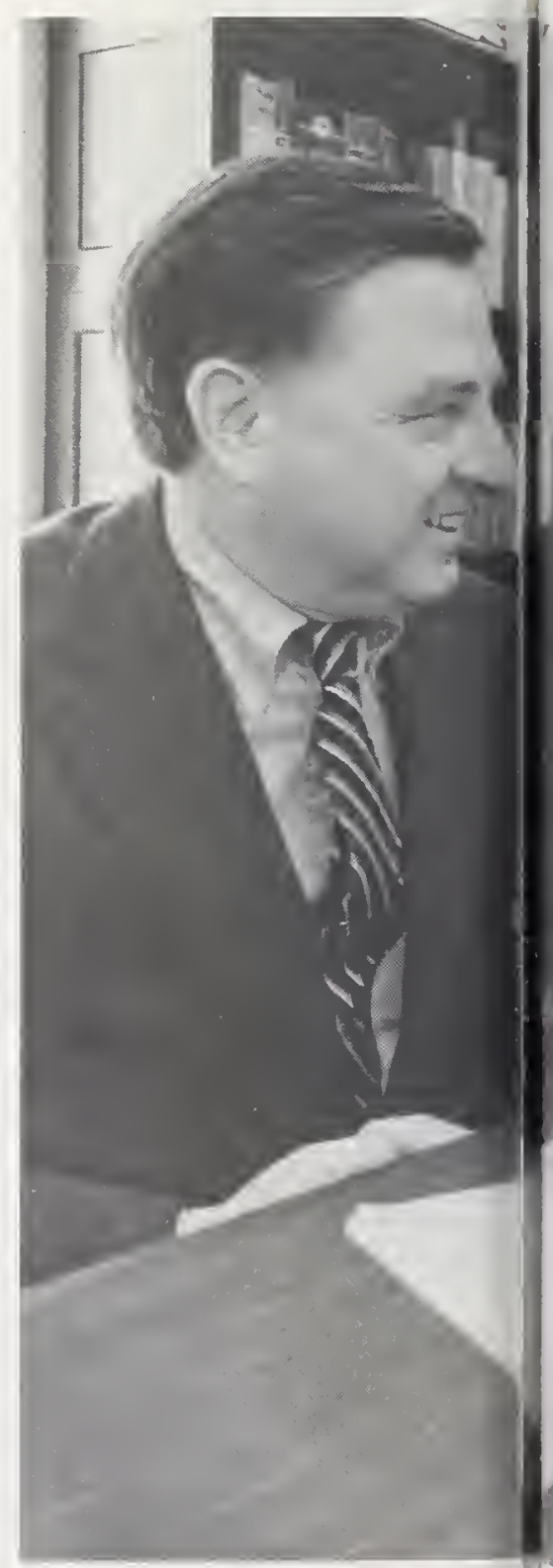




\section{National Center for Standards and Certification Information}

The National Center for Standards and Certification Information (NBS-NCSCI) maintains a reference collection of some 240,000 engineering and related standards issued by U.S. technical societies, professional organizations, and trade associations; State purchasing offices; U.S. Federal Government agencies; and major foreign national and international standardizing bodies. NBS-NCSCI publishes general and special indexes of standards. Information services which are free consist of searching Key-Word-In-Context (KWIC) Indexes to determine whether there are any published standards, specifications, codes, test methods, or recommended practices for a given item or product. Inquiries should be directed to:

National Center for Standards and Certification Information

Room B166, Technology Building

National Bureau of Standards

Washington, DC 20234

(301) $921-2587$

\section{National Standard Reference Data System}

The National Standard Reference Data System (NSRDS) is a nationwide program established to compile and critically evaluate quantitative physical science data and assure its availability to the technical community. The program publishes compilations of critically evaluated data, critical reviews of experimental techniques, and bibliographies. A complete listing of the publications of the NSRDS is available from the Office of Standard Reference Data (OSRD). The OSRD responds in a limited way to queries within the scope of the program by providing references, referrals, documentation, or data, as available. The program's bimonthly newsletter is available on request. Inquiries or requests for further information should be directed to:

Office of Standard Reference Data

A323, Physics Building

National Bureau of Standards

Washington, DC 20234

(301) 921.2104

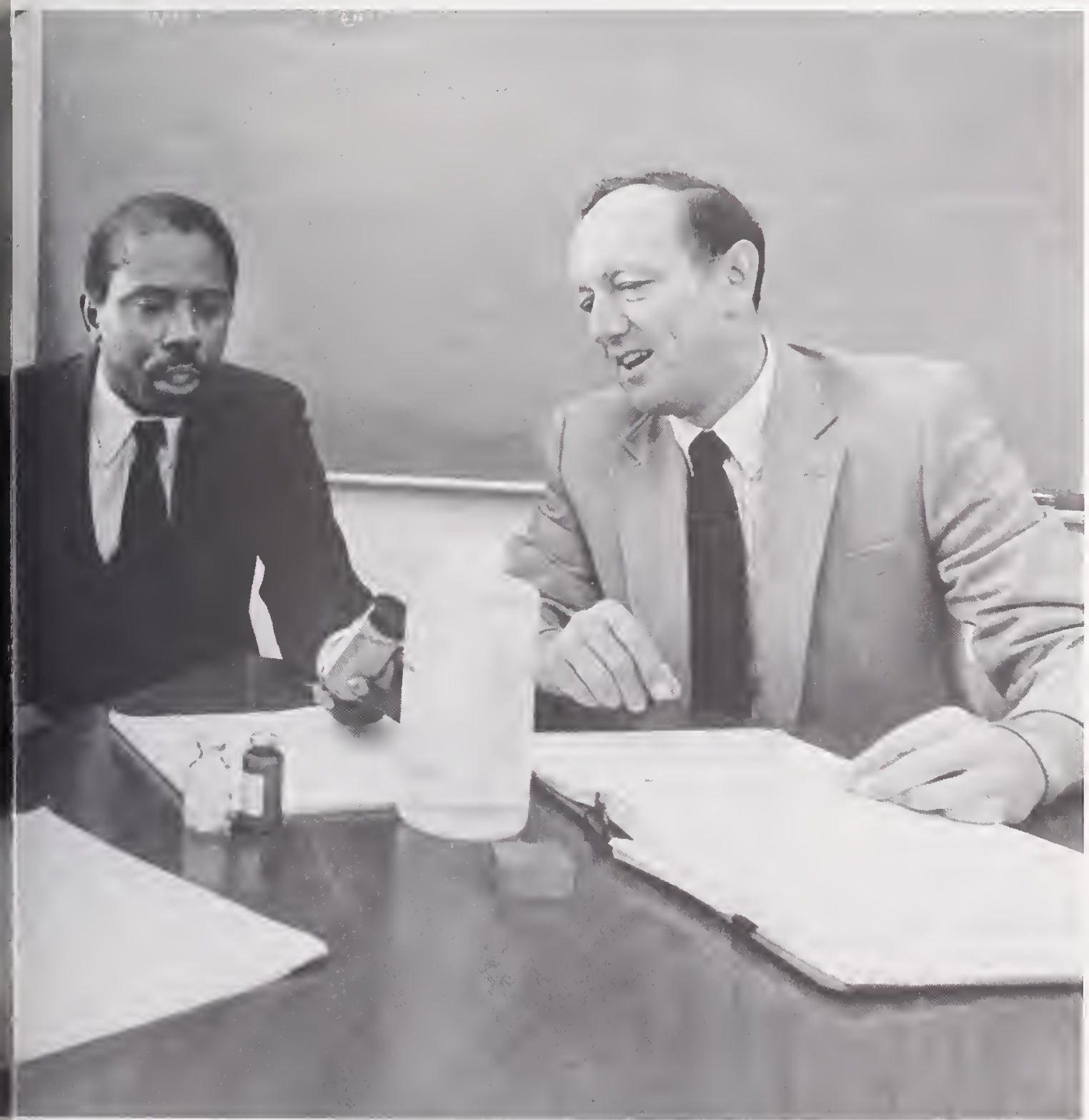

Foreign orders are processed by Ruth Meyer, who also answers inquiries regarding foreign shipments and overdue accounts.
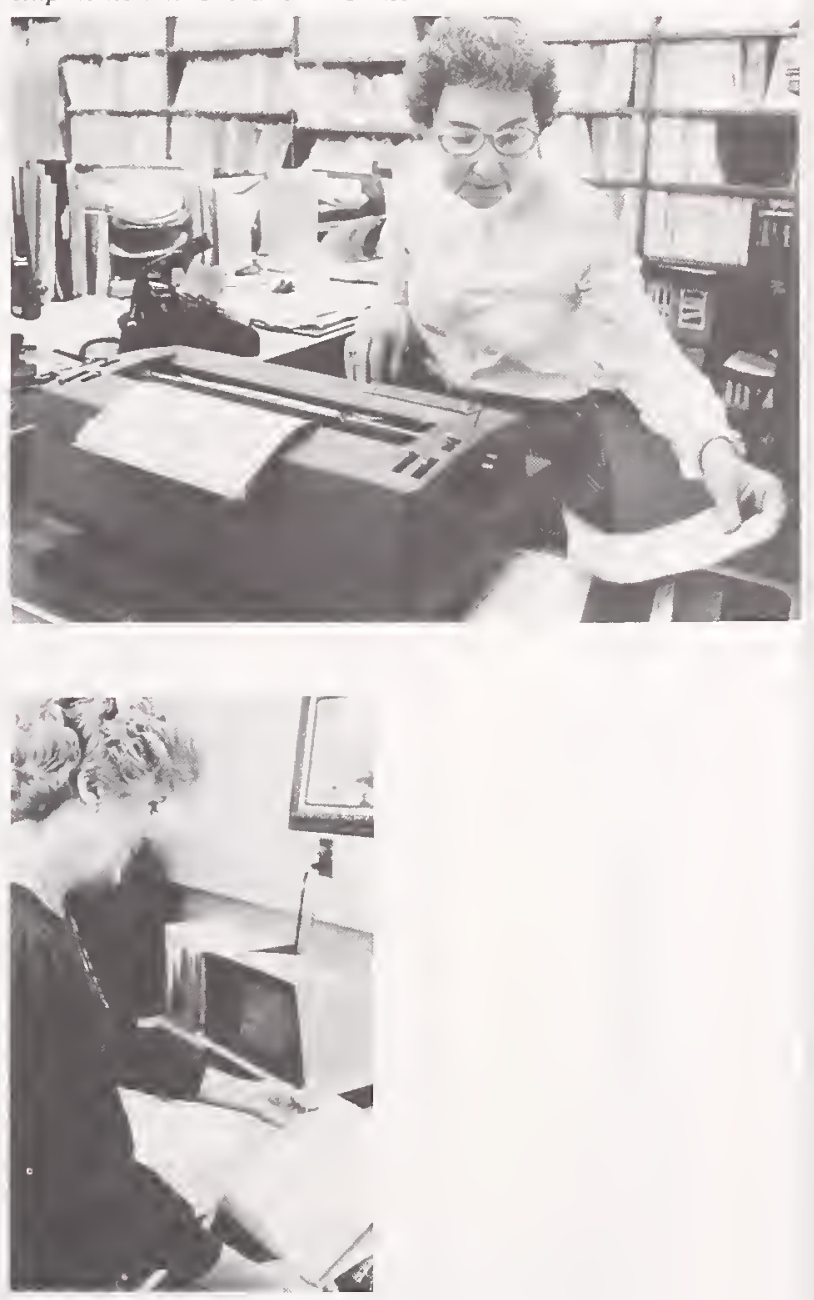

The management of the computer system is handled by Lee Klein, data processing manager. This system is able to process a normal customer order in three days. 


\section{Guide of Requesting Development of Standard Reference Materials}

The National Bureau of Standards has the function to develop, produce, and distribute Standard Reference Materials (SRM's) that provide a basis for comparison of measurements on materials and that aid in the control of production processes. To perform this function, the Office of Standard Reference Materials evaluates the requirements of science, industry, and government for carefully characterized reference materials, and directs their production and distribution.

NBS currently has over 1000 SRM's available, about 100 new ones in preparation, and requests for the preparation of many others. below.

In developing an NBS-SRM, the candidate material must meet one or more of the criteria listed

1. The SRM must permit users to attain more accurate measurements.

2. The production of the SRM elsewhere is not economically or technically feasible.

3. The SRM would be an industry-wide standard for commerce from a neutral source not otherwise available.

4. NBS production of the SRM would provide continued availability from a common source for a highly characterized material that is important to science, industry, or government.

NBS has recognized and responded to the need to enlarge the scope of the program to include all types of well-characterized materials that can be used to calibrate a measurement system or to produce scientific data that can be readily referred to a common base. However, the demand for new SRM's greatly exceeds the Bureau's capacity to produce and certify these materials. Consequently, requests for new SRM's that would have limited use, or for which the need is not very great, are deferred in favor of requests that clearly show a critical need. To determine which requests are to receive top priority, NBS needs and heavily relies upon the information supplied by industry, either through its own representatives or through interested organizations, such as the American National Standards Institute, American Nuclear Society, American Petroleum Institute, American Society for Testing and Materials, etc.

Accordingly, while the Bureau welcomes all requests for the development of new SRM's, both the Bureau and industry would be helped if such requests are accompanied by information that will permit an objective assessment of the urgency and importance of proposed new reference materials.

Requests for the development and new Standard Reference Materials should include as much of the information listed below as possible.

1. Short title of the proposed Standard Reference Material.

2. Purpose for which the SRM would be used.

3. Reasons why the SRM is needed.

4. Special characteristics and/or requirements for the material. Include additional requirements and reasons, if more than one SRM is necessary for standardization in this area.

5. Your estimate of the possible present and future (6-10 year) demand for such an SRM in your own operations and elsewhere. (National and international estimates are very useful.)

6. Whether such an SRM, or a similar one, can be produced by, or obtained from a source other than NBS. If so, give reasons to justify its preparation by NBS.

7. Miscellaneous pertinent information to aid justification for the SRM, such as: (a) an estimate of the range of application, monetary significance of the measurement affected, and scientific and/or technological significance including, when feasible, estimates of the impact upon industrial productivity or growth, and (b) supporting letters from industry leaders, trade organizations, interested committees, and others.

Requests should be sent to:

Office of Standard Reference Materials

National Bureau of Standards

B311, Chemistry Building

Washington, DC 20234

Dolly Downs prepares the certificates that are supplied with each SRM. This certificate provides the customer with all pertinent information about the SRM, its intended use, and method of certification.

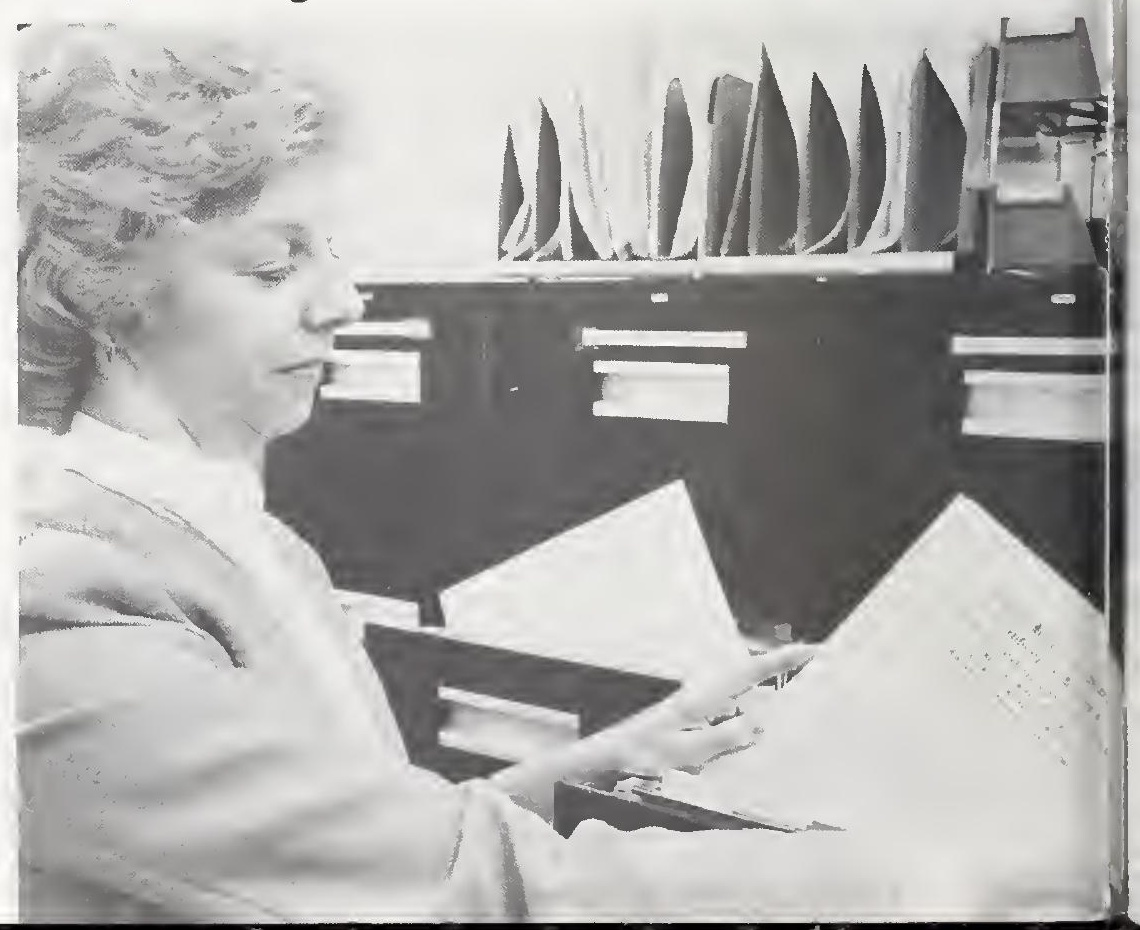




\section{Certified Reference Materials From Other Sources}

Sources of certified reference materials (CRM's) are now world wide. The International Organization for Standardization (ISO), through its Council Committee on Reference Materials (REMCO), has prepared an international Directory of Certified Reference Materials. Inquiries may be directed to:

Dr. M. Parkany

Secretary for REMCO

International Organization for Standardization

1, Rue de Varembe

Case Postale 56

1211 Geneva 20

Switzerland

In the period 1972-83, the International Union of Pure and Applied Chemistry (IUPAC), through its Commission on Physicochemical Measurements and Standards, prepared and issued a catalog of CRM's that are useful for the realization of physicochemical properties. It also has prepared a number of related documents. Information may be obtained by writing:

Dr. K. N. Marsh

Chairman, IUPAC Commission I.4

Physicochemical Measurements and Standards

The University of New England

Armidale, NSW

Post Code 2361

Australia

The current IUPAC edition is: Physicochemical Measurements: Catalogue of Reference Materials from National Laboratories, Revised 1976, Pure \& Appl. Chem., 48, 503-414 (1976).

Major government and national laboratories which supply CRM's are listed below:

\begin{tabular}{|ll}
\hline Country & National Laboratory \\
\hline Australia & Commonwealth Scientific and Industrial Research Organization \\
& National Standards Laboratory \\
& University Grounds, City Road \\
& Chippendale, NSW 2008 \\
& Australia \\
\hline Brazil & Instituto de Pesiquisas Technologicas do Estado de Sao Paulo \\
& S/A-IPT \\
& Divisao de Quimica e Engenharia Quimica \\
& Nucleo de Padroes Analiticos \\
& Caixa Postal 7141 \\
& 01000 Sao Paulo SP \\
& Brazil \\
\hline Canada & Canadian Certified Reference Materials Project \\
& C/O Mineral Sciences Laboratories, CAMNET \\
Canada Centre for Mineral and Energy Technology & 555 Booth Street \\
& Ottawa, Ontario K 1A OG1 \\
Canada \\
\hline Bureau National de Metrologie (B.N.M.) \\
8-10, Rue Crillon \\
75194 Paris Cedex 04 \\
France \\
or \\
Srance \\
Service des Materiaux de Reference \\
(S.M.R.) \\
1, Rue Gaston Boissier \\
75015 Paris \\
France \\
\hline
\end{tabular}




\section{(Continued)}

\begin{tabular}{|c|c|}
\hline \multirow[t]{2}{*}{ Germany (West) } & $\begin{array}{l}\text { Bundesanstalt fur Materialprufung (BAM) } \\
\text { Unter den Eichen } 87 \\
\text { D-1000 Berlin } 45 \\
\text { Germany, F.R. }\end{array}$ \\
\hline & $\begin{array}{l}\text { Physikalisch-Technishe Bundesanstalt (PTB) } \\
\text { Bundesallee } 100 \\
\text { D-3300 Braunschweig } \\
\text { Germany, F.R. }\end{array}$ \\
\hline Hungary & $\begin{array}{l}\text { National Office of Measures } \\
\text { P.O. Box } 19 \\
\text { H-1531 Budapest } \\
\text { Hungary }\end{array}$ \\
\hline$\overline{\text { Japan }}$ & $\begin{array}{l}\text { Standards Department } \\
\text { Agency of Industrial Science and Technology } \\
\text { Ministry of International Trade and Industry } \\
\text { 3-1, Kasumigaseki 1, Chiyodaku, } \\
\text { Tokyo } \\
\text { Japan }\end{array}$ \\
\hline \multirow[t]{2}{*}{ Netherlands } & $\begin{array}{l}\text { Institute for Physical Chemistry TNO } \\
\text { Utrechtseweg } 48, \\
\text { P.O. Box } 108 \\
\text { Zeist } \\
\text { Netherlands }\end{array}$ \\
\hline & $\begin{array}{l}\text { Rijks Institute voor Volkgezondheid } \\
\text { P.O. Box } 1 \\
\text { Bilthoven } \\
\text { Netherlands }\end{array}$ \\
\hline$\overline{\text { Poland }}$ & $\begin{array}{l}\text { Division of Physico-Chemical Metrology } \\
\text { National Board for Quality Control and Measures } \\
\text { 2, Elektoralna Street } \\
\text { Warsaw } \\
\text { Poland }\end{array}$ \\
\hline South Africa & $\begin{array}{l}\text { South African Bureau of Standards } \\
\text { Private Bag X191 } \\
\text { Pretoria, Transvaal } 0001 \\
\text { Republic of South Africa } \\
\end{array}$ \\
\hline \multirow[t]{2}{*}{ United Kingdom } & $\begin{array}{l}\text { National Physical Laboratory } \\
\text { Office of Reference Materials } \\
\text { Teddington, Middlesex TW11 OLW } \\
\text { United Kingdom }\end{array}$ \\
\hline & $\begin{array}{l}\text { British Standards Institution } \\
10 \text { Blackfriars Street } \\
\text { Manchester, M3 5DT } \\
\text { United Kingdom }\end{array}$ \\
\hline \multirow[t]{3}{*}{ United States } & $\begin{array}{l}\text { National Bureau of Standards } \\
\text { Office of Standard Reference Materials } \\
\text { B311, Chemistry Building } \\
\text { Washington, DC } 20234 \\
\text { USA }\end{array}$ \\
\hline & $\begin{array}{l}\text { U.S. Department of Energy } \\
\text { New Brunswick Laboratory } \\
9800 \text { South Cass Avenue, D-350 } \\
\text { Argonne, IL } 60439 \\
\text { USA }\end{array}$ \\
\hline & $\begin{array}{l}\text { Center for Disease Control } \\
\text { Atlanta, GA } 30333 \\
\text { USA }\end{array}$ \\
\hline
\end{tabular}




\section{(Continued)}

\begin{tabular}{ll|}
\hline USSR & Gosstandart \\
9, Leninsky Prospekt \\
117049 Moscow \\
USSR \\
\hline Community Bureau of Reference (BCR) \\
Directorate General XII, CEE \\
200, Rue de la Loi \\
B-1049 Brussels \\
Belgium \\
Central Bureau of Nuclear Measurements \\
Commission of the European Communities \\
Geel Establishment \\
Steenweg op Retie \\
B-2440 Geel \\
Belgium \\
International Atomic Energy Agency \\
Analytical Quality Control Services \\
Laboratory Seibersdorf \\
P.O. Box 590 \\
A-1011 Vienna \\
Austria \\
WHO Collaborating Centre for Chemical \\
Reference Substances \\
Apotekens Centrallaboratorium \\
Box 3045 \\
S.171 03 Solna \\
Sweden \\
World Health Organization \\
CH-1211 Geneva 27 \\
Switzerland
\end{tabular}

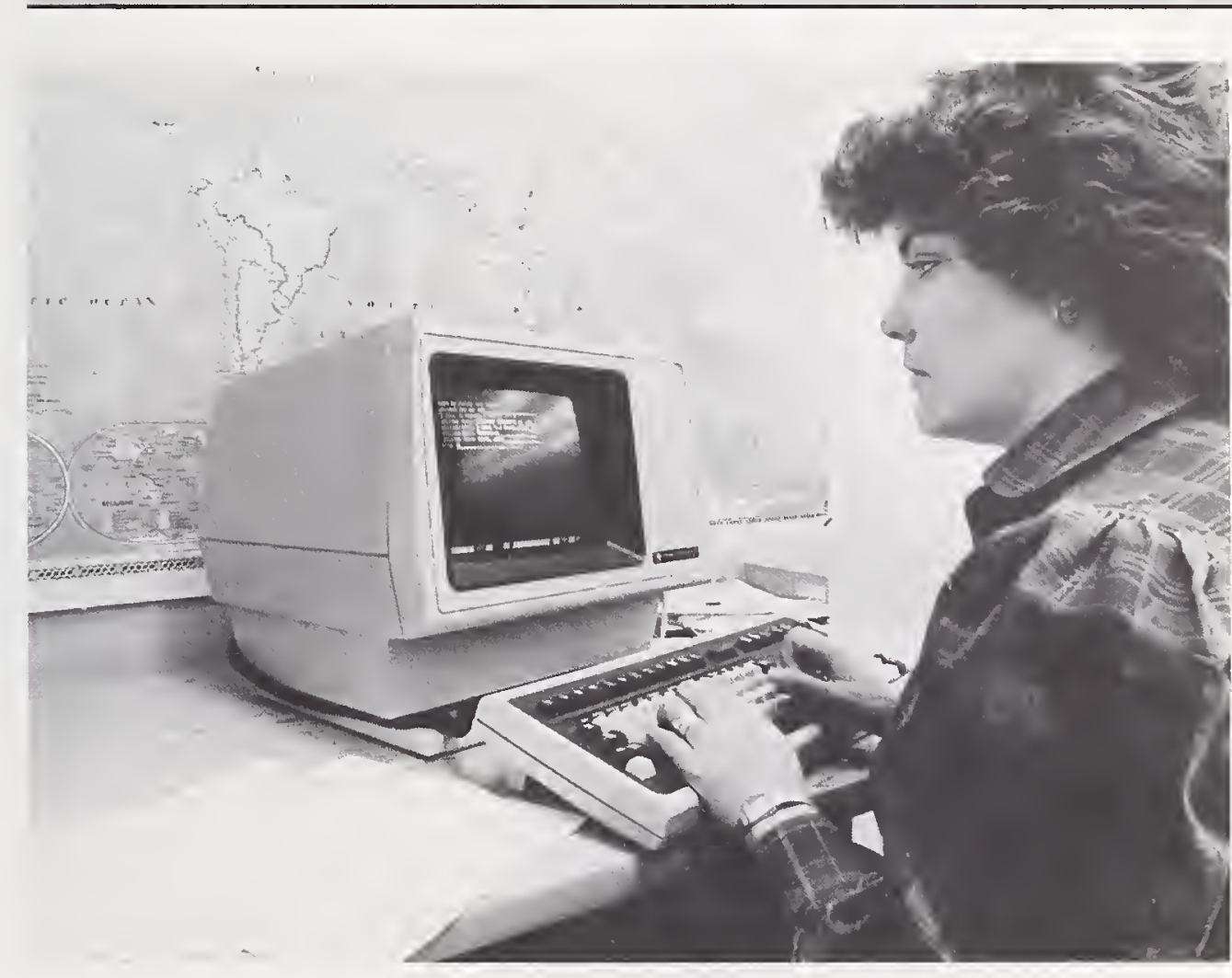

Claudia Engel processes foreign orders, handles paperwork for special methods of payment, and prepares invoices for mailing to customers. 


\section{Other NBS Publications in the 260 Series}

Hudson, C. H., ed., Catalog of NBS Standard Reference Materials (1984. 85 edition), NBS Spec. Publ. 260 (January 1984).

Michaelis, R. E., and Wyman, L. L., Standard Reference Materials: Preparation of White Cast Iron Spectrochemical Standards, NBS Misc. Publ. 260-1 (June 1964). COM74-11061**

Michaelis, R. E., Wyman, L. L., and Flitsch, R., Standàrd Reference Materials: Preparation of NBS Copper-Base Spectrochemical Standards, NBS Misc. Publ. 260-2 (October 1964). COM7411063**

Michaelis, R. E., Yakowitz, H., and Moore, G. A., Standard Reference Materials: Metallographic Characterization of an NBS Spectrometric LowAlloy Steel Standard, NBS Misc. Publ. 260-3 (October 1964). COM74-11060**

Hague, J. L., Mears, T. W., and Michaelis, R. E., Standard Reference Materials: Sources of Information, NBS Misc. Publ. 260-4 (February 1965). COM74-11059

Alvarez, R., and Flitsch, R., Standard Reference Materials: Accuracy of Solution X-Ray Spectrometric Analysis of Copper-Base Alloys, NBS Misc. Publ. 260.5 (March 1965). PB168068**
Shultz, J. I., Standard Reference Materials: Methods for the Chemical Analysis of White Cast Iron Standards, NBS Misc. Publ. 260-6 (July 1965). COM74-11068** Bell, R. K., Standard Reference Materials: Methods for the Chemical Analysis of NBS Copper-Base Spectrochemical Standards, NBS Misc. Publ. 260-7 (October 1965). COM74-11067**

Richmond, M. S., Standard Reference Materials: Analysis of Uranium Concentrates at the National Bureau of Standards, NBS Misc. Publ. 260-8 (December 1965). COM74-11066** Anspach, S. C., Cavallo, L. M., Garfinkel, S. B., Hutchinson, J. M. R., and Smith, C. N., Standard Reference Materials: Half Lives of Materials Used in the Preparation of Standard Reference Materials of Nineteen Radioactive Nuclides Issued by the National Bureau of Standards, NBS Misc. Publ. 260-9 (November 1965). COM74-11065**

Yakowitz, H., Vieth, D. L., Heinrich, K. F. J., and Michaelis, R. E., Standard Reference Materials: Homogeneity Characterization of NBS Spectrometric Standards II: Cartridge Brass and Low-Alloy Steel, NBS Misc. Publ. 260.10 (December 1965). COM7411064**

Napolitano, A., and Hawkins, E. G., Standard Reference Materials:

Viscosity of Standard Lead-Silica

Glass, NBS Misc. Publ. 260-11 (November 1966). NBS Misc. Publ. 260-11**

Yakowitz, H., Vieth, D. L., and Michaelis, R. E., Standard Reference Materials: Homogeneity Charac. terization of NBS Spectrometric Standards III: White Cast Iron and Stainless Steel Powder Compact, NBS Misc. Publ. 260-12 (September, 1966). NBS Misc. Publ. 260-12**
Spijkerman, J. L., Snediker, D. K., Ruegg, F. C., and DeVoe, J. R., Standard Reference Materials: Mossbauer Spectroscopy Standard for the Chemical Shift of Iron Compounds, NBS Misc. Publ. 260-13 (July 1967). NBS Misc. Publ. 260$13 * *$

Menis, O., and Sterling, J. T., Standard Reference Materials: Determination of Oxygen in Ferrous Materials - SRM 1090, 1091, and 1092, NBS Misc. Publ. 260-14 (September 1966). NBS Misc. Publ. 260-14**

Passaglia, E., and Shouse, P. J., Standard Reference Materials: Recommended Method of Use of Standard Light-Sensitive Paper for Calibrating Carbon Arcs Used in Testing Textiles for Colorfastness to Light, NBS Misc. Publ. 260-15 (June 1967). (Replaced by NBS Spec. Publ. 260-41.)

Yakowitz, H., Michaelis, R. E., and Vieth, D. L., Standard Reference Materials: Homogeneity Characterization of NBS Spectrometric Standards IV: Preparation and Microprobe Characterization of W-20\% MO Alloy Fabricated by Powder Metallurgical Methods, NBS Spec. Publ. 260-16 (January 1969). COM74-11062**

Catanzaro, E. J., Champion, C. E., Garner, E. L., Marinenko, G., Sappenfield, K. M., and Shields, W. R. Standard Reference Materials: Boric Acid; Isotopic and Assay Standard Reference Materials, NBS Spec. Publ. 260.17 (February 1970). Out of Print. 
Geller, S. B., Mantek, P. A., and Cleveland, N. G., Standard Reference Materials: Calibration of NBS Secondary Standard Magnetic Tape (Computer Amplitude Reference) Using the Reference Tape Amplitude Measurement "Process A", NBS Spec. Publ. 260-18 (November 1969). (See NBS Spec. Publ. 260-29.)

Paule, R. C., and Mandel, J., Standard Reference Materials: Analysis of Interlaboratory Measurements on the Vapor Pressure of Gold (Certification of Standard Reference Material 745). NBS Spec. Publ. 260-19 (January 1970). PB 190071**

Paule, R. C., and Mandel, J., Standard Reference Materials: Analysis of Interlaboratory Measurements on the Vapor Pressures of Cadmium and Silver, NBS Spec. Publ. 260-21 (January 1971). COM74-11359**

Yakowitz, H., Fiori, C. E., and Michaelis, R. E., Standard Reference Materials: Homogeneity Characterization of Fe-3 Si Alloy, NBS Spec. Publ. 260.22 (February 1971). COM74-11357**

Napolitano, A., and Hawkins, E. G., Standard Reference Materials: Viscosity of a Standard Borosilicate Glass, NBS Spec. Publ. 260-23 (December 1970). COM71-00157**

Sappenfield, K. M., Marinenko, G., and Hague, J. L., Standard Reference Materials: Comparison of Redox Standards, NBS Spec. Publ. 260-24 (January 1972). COM72-50058**

Hicho, G. E., Yakowitz, H., Rasberry, S. D., and Michaelis, R. E., Standard Reference Materials: A Standard Reference Material Containing Nominally Four Percent Austenite, NBS Spec. Publ. 260-25 (February 1971). COM74-11356**

Martin, J. F., Standard Reference Materials: National Bureau of Standards-US Steel Corporation Joint Program for Determining Oxygen and Nitrogen in Steel, NBS Spec. Publ. 260-26 (February 1971). SN003. 00300786-2*

Garner, E. L., Machlan, L. A., and Shields, W. R., Standard Reference Materials: Uranium Isotopic Standard Reference Materials, NBS Spec. Publ. 260-27 (April 1971). COM74-11358**
Heinrich, K. F. J., Myklebust, R. L., Rasberry, S. D., and Michaelis, R. E., Standard Reference Materials: Preparation and Evaluation of SRM's 481 and 482 GoldSilver and Gold. Copper Alloys for Microanalysis, NBS Spec. Publ. 260-28 (August 1971). COM71-50365**

Geller, S. B., Standard Reference Materials: Calibration of NBS Secondary Standard Magnetic Tape (Computer Amplitude Reference) Using the Reference Tape Amplitude Measurement "Process A-Model 2," NBS Spec. Publ. 260-29 (June 1971). COM71.50282

Gorozhanina, R. S., Freedman, A. Y., and Shaievitch, A. B. (translated by M. C. Selby), Standard Reference Materials: Standard Samples Issued in the USSR (A Translation from the Russian). NBS Spec. Publ. 260-30 (June 1971). COM71-50283**

Hust, J. G., and Sparks, I. L., Standard Reference Materials: Thermal Conductivity of Electrolytic Iron SRM 734 from 4 to $300 \mathrm{~K}$, NBS Spec. Publ. 260-31 (November 1971). COM7150563**

Mavrodineanu, R., and Lazar, J. W., Standard Reference Materials: Standard Quartz Cuvettes, for High Accuracy Spectrophotometry, NBS Spec. Publ. 260-32 (December 1973). SN003-003-01213-1*

Wagner, H. L., Standard Reference Materials: Comparison of Original and Supplemental SRM 705, Narrow Molecular Weight Distribution Polystyrene, NBS Spec. Publ. 260-33 (May 1972). COM72-50526**

Sparks, L. L., and Hust, J. G., Standard Reference Materials: Thermoelectric Voltage, NBS Spec. Publ. 260-34 (April 1972). COM72. $50371^{* *}$

Sparks, L. L., and Hust, J. G., Standard Reference Materials: Thermal Conductivity of Austenitic Stainless Steel, SRM 735 from 5 to 280 K, NBS Spec. Publ. 260-35 (April 1972.) COM72-50368**

Cali, J. P., Mandel, J., Moore, L. J., and Young, D. S., Standard Reference Materials: A Reference Method for the Determination of Calcium in Serum, NBS SRM 915, NBS Spec. Publ. 260. 36 (May 1972). COM72.50527**

Shultz, J. I., Bell, R. K., Rains, T. C., and Menis, O., Standard Reference Materials: Methods of Analysis of NBS Clay Standards, NBS Spec. Publ. 260-37 (June 1972). COM72. $50692 * *$
Richmond, J. C., and Hsia, J. J., Standard Reference Materials: Preparation and Calibration of Standards of Spectral Specular Reflectance, NBS Spec. Publ. 260-38 (May 1972). COM72-50528**

Clark, A. F., Denson, V. A., Hust, J. G., and Powell, R. L., Standard Reference Materials: The Eddy Current Decay Method for Resistivity Characterization of High Purity Metals, NBS Spec. Publ. 26039 (May 1972). COM72-50529**

McAdie, H. G., Garn, P. D., and Menis, O., Standard Reference Materials: Selection of Thermal Analysis Temperature Standards Through a Cooperative Study (SRM 758, 759, 760), NBS Spec. Publ. 260. 40 (August 1972). COM72-50776**

Wood, L. A., and Shouse, P. J., Standard Reference Materials: Use of Standard Light-Sensitive Paper for Calibrating Carbon Arcs Used in Testing Textiles for Colorfastness to Light, NBS Spec. Publ. 260-41 (August 1972). COM72-50775** Wagner, H. L., and Verdier, P. H., eds., Standard Reference Materials: The Characterization of Linear Polyethylene, SRM 1475, NBS Spec Publ. 260-42 (September 1972). COM7250944**

Yakowitz, H., Ruff, A. W., and Michaelis, R. E., Standard Reference Materials: Preparation and Homogeneity Characterization of an Austenitic Iron-Chromium-Nickel Alloy, NBS Spec. Publ. 260-43 (November 1972). COM73-50760** Schooley, J. F., Soulen, R. J., Jr., and Evans, G. A., Jr., Standard Reference Materials: Preparation and Use of Superconductive Fixed Point Devices, SRM 767, NBS Spec. Publ. 260-44 (December 1972). COM73 $50037 * *$

Greifer, B., Maienthel, E. J., Rains, T. C., and Rasberry, S. D., Standard Reference Materials: Powdered Lead. Based Paint, SRM 1579, NBS Spec. Publ. 260-45 (March 1973). COM73. $50226^{* *}$ 
Hust, J. G., and Giarratano, P. J., Standard Reference Materials: Thermal Conductivity and Electrical Resistivity Standard Reference Materials: Austenitic Stainless Steel, SRM's 735 and 798, from 4 to 1200 K, NBS Spec. Publ. 260.46 (March 1975). SN003-003-01278-5*

Hust, J. G., Standard Reference Materials: Electrical Resistivity of Electrolytic Iron, SRM 797, and A ustenitic Stainless Steel, SRM 798, from 5 to $280 \mathrm{~K}$, NBS Spec. Publ. 26047 (February 1974). COM74. $50176^{* *}$

Mangum, B. W., and Wise, J. A., Standard Reference Materials: Description and Use of Precision Thermometers for the Clinical Laboratory, SRM 933 and SRM 934, NBS Spec. Publ. 260-48 (May 1974). SN003-003-01278-5*

Carpenter, B. S., and Reimer, G. M., Standard Reference Materials: Calibrated Glass Standards for Fission Track Use, NBS Spec. Publ. 260-49 (November 1974). SN003-00301344.7*

Hust, J. G., and Giarratano, P. J., Standard Reference Materials: Thermal Conductivity and Electrical Resistivity Standard Reference Materials: Electrolytic Iron, SRM's 734 and 797 from 4 to $1000 \mathrm{~K}$, NBS Spec. Publ. 260-50 (June 1975). SN003-003-01425-7*
Mavrodineanu, R., and Baldwin, J. R., Standard Reference Materials: Glass Filters As a Standard Reference Material for Spectrophotometry; Selection; Preparation; Certification; Use-SRM 930, NBS Spec. Publ. 26051 (November 1975). SN003-003. $01481.8^{*}$

Hust, J. G., and Giarratano, P. J., Standard Reference Materials:

Thermal Conductivity and Electrical Resistivity Standard Reference Materials 730 and 799 , from 4 to 3000 K, NBS Spec. Publ. 260.52 (September 1975). SN003-003-01464. $8^{*}$

Durst, R. A., Standard Reference Materials: Standardization of $\mathrm{pH}$ Measurements, NBS Spec. Publ. 260. 53 (December 1975, Revised). SN003. 003-01551-2*

Burke, R. W., and Mavrodineanu, R., Standard Reference Materials: Certification and Use of Acidic Potassium Dichromate Solutions as an Ultraviolet Absorbance Standard, NBS Spec. Publ. 260-54 (August 1977). SN003-003-01828-7*

Ditmars, D. A., Cezairliyan, A., Ishihara, S., and Douglas, T. B., Standard Reference Materials: Enthalpy and Heat Capacity; Molybdenum SRM 781, From 273 to 2800 K, NBS Spec. Publ. 260.55 (September 1977). SN003-003-01836. $8^{*}$

Powell, R. L., Sparks, L. L., and Hust, J. G., Standard Reference Materials: Standard Thermocouple Material, Pt.27: SRM 1967, NBS Spec. Publ. 26056 (February 1978). SN0003-003018864*

Cali, J. P. and Plebanski, T., Guide to United States Reference Materials, NBS Spec. Publ. $260-57$ (February 1978). SN003-003-01883-0*

Barnes, J. D. and Martin, G. M., Standard Reference Materials: Polyester Film for Oxygen Gas Transmission Measurements. SRM 1470, NBS Spec. Publ. 260-58 (June 1979). SN003-003-02077-0*

Chang, T. and Kahn, A. H., Standard Reference Materials: Electron Paramagnetic Resonance Intensity Standard: SRM 2601, NBS Spec. Publ. 260-59 (August 1978). SN003. $003-01975-5^{*}$
Velapoldi, R. A., Paule, R. C., Schaffer, R., Mandel, J., and Moody, J. R., Standard Reference Materials: A Reference Method for the Determination of Sodium in Serum, NBS Spec. Publ. 260-60 (A ugust 1978). SN003-003-01978-0*

Verdier, P. H., and Wagner, H. L., Standard Reference Materials: The Characterization of Linear Polyethylene (SRM 1482, 1483, 1484), NBS Spec. Publ. 260-61 (December 1978). SN003-003.02006$1^{*}$

Soulen, R. J., and Dove, R. B., Standard Reference Materials: Temperature Reference Standard for Use Below 0.5 K (SRM 768). NBS Spec. Publ. 260-62 (April 1979). SN003-003-02047.8*

Velapoldi, R. A., Paule, R. C., Schaffer, R., Mandel, J., Machlan, L. A., and Gramlich, J. W., Standard Reference Materials: A Reference Method of the Determination of Potassium in Serum. NBS Spec. Publ. 260-63 (May 1979). SN003-003-02068* Velapoldi, R. A., and Mielenz, K. D., Standard Reference Materials: A Fluorescence Standard Reference Material Quinine Sulfate Dihydrate (SRM 936), NBS Spec. Publ. 260-64 (January 1980). SN003-003-02148-2*

Marinenko, R. B., Heinrich, K. F. J., and Ruegg, F. C., Standard Reference Materials: Micro-Homogeneity Studies of NBS Standard Reference Materials, NBS Research Materials, and Other Related Samples. NBS Spec. Publ. 260-65 (September 1979). SN003-003-02114-1*

Venable, W. H., Jr., and Eckerle, K. L., Standard Reference Materials: Didymium Glass Filters for Calibrating the Wavelength Scale of Spectrophotometers (SRM 2009. 2010, 2013). NBS Spec. Publ. 260-66 (October 1979). SNOO3-003-02127-0* 
Velapoldi, R. A., Paule, R. C., Schaffer, R., Mandel, J., Murphy, T. J., and Gramlich, J. W., Standard Reference Materials: A Reference Method for the Determination of Chloride in Serum, NBS Spec. Publ. 260-67 (November 1979). SN003-00302136-9*

Mavrodineanu, R. and Baldwin, J. R., Standard Reference Materials: MetalOn-Quartz Filters as a Standard Reference Material for Spectrophotometry-SRM 2031, NBS Spec. Publ. 260-68 (April 1980). SN003-00302167.9*

Velapoldi, R. A., Paule, R. C., Schaffer, R., Mandel, J., Machlan, L. A., Garner, E. L., and Rains, T. C., Standard Reference Materials: A Reference Method for the Determination of Lithium in Serum, NBS Spec. Publ. 260-69 (July 1980). SN003-003-02214.4*

Marinenko, R. B., Biancaniello, F. Boyer, P. A., Ruff, A. W., DeRobertis, L., Standard Reference Materials: Preparation and Characterization of an Iron-Chromium-Nickel Alloy for Microanalysis, NBS Spec. Publ. 260 70 (May 1981). SN003-003-02328-1*

Seward, R. W., and Mavrodineanu, R. Standard Reference Materials: Summary of the Clinical Laboratory Standards Issued by the National Bureau of Standards, NBS Spec. Publ. 260-71 (November 1981). SN003-003-02381.7*

Reeder, D. J., Coxon, B., Enagonio, D., Christensen, R. G., Schaffer, R., Howell, B. F., Paule, R. C., Mandel, J., Standard Reference Materials: SRM 900, Antiepilepsy Drug Level Assay Standard, NBS Spec. Publ. 260-72 (June 1981). SN003-003-02329. 9*

Interrante, C. G., and Hicho, G. E. Standard Reference Materials: A Standard Reference Material Containing Nominally Fifteen Percent Austenite (SRM 486), NBS Spec. Publ. 260-73 (January 1982). SN003-003-02386-8*
Marinenko, R. B., Standard

Reference Materials: Preparation and Characterization of K-411 and K-414 Mineral Glasses for Microanalysis: SRM 470. NBS Spec. Publ. 260-74 (April 1982). SN003-003-023-95-7*

Weidner, V. R., Hsia, J. J., Standard Reference Materials: Preparation and Calibration of First Surface Aluminum Mirror Specular Reflectance Standards (SRM 2003a), NBS Spec. Publ. 260.75 (May 1982). SN003-003-023-99-0*

Hicho, G. E. and Eaton, E. E., Standard Reference Materials: A Standard Reference Material Containing Nominally Five Percent Austenite (SRM 485a), NBS Spec. Publ. 260.76 (August 1982).*

Furukawa, G. T., Riddle, J. L., Bigge, W. G., and Pfieffer, E. R., Standard Reference Materials: Application of Some Metal SRM's as Thermometric Fixed Points, NBS Spec. Publ. 260-77 (August 1982).*

Hicho, G. E. and Eaton, E. E., Standard Reference Materials: Standard Reference Material Containing Nominally Thirty Percent Austenite (SRM 487), NBS Spec. Publ. 260-78 (September 1982). SN003-003-024-35-0*

Richmond, J. C., Hsia, J. J., Weidner, V. R., and Wilmering, D. B., Standard Reference Materials: Second Surface Mirror Standards of Specular Spectral Reflectance (SRM's 2023 , 2024, 2025), NBS Spec. Publ. 260-79 (October 1982). SN003-003-024-47-3* Schaffer, R., Mandel, J., Sun, T., Cohen, A., and Hertz, H. S., Standard Reference Materials: Evaluation by an ID/MS Method of the AACC Reference Method for Serum Glucose, NBS Spec. Publ. 260-80 (October 1982). SN003-003-024-43-1 *

Burke, R. W., Mavrodineanu, R. (NBS retired). Standard Reference

Materials: Accuracy in Analytical Spectrophotometry, NBS Spec. Publ 260-81 (April 1983). SN003-003-02484-8*

Weidner, V.R. Standard Reference Materials: White Opal Glass Diffuse Spectral Reflectance Standards for the Visible Spectrum (SRM's 2015 and 2016). NBS Spec. Publ. 260-82 (April 1983). SN-003-003024-89.9*
Bowers, G. N., Jr., Alvarez, R., Cali, J. P. (NBS retired), Eberhardt, K. R. Reeder, D. J., Schaffer, R., Uriano, G. A., Standard Reference Materials: The Measurement of the Catalytic (Activity) Concentration of Seven Enzymes in NBS Human Serum SRM 909. NBS Spec. Publ. 260.83 (June 1983). SN003-003-024-99.6*

Gills, T. E., Seward, R. W. Collins, R. J., and Webster, W. C., Standard Reference Materials: Sampling, Materials Handling, Processing, and Packaging of NBS Sulfur in Coal Standard Reference Materials, 2682. 2683, 2684 and 2685. NBS Spec. Publ. 260-84 (In Press).

Swyt, D. A., Standard Reference Materials: A Look at Techniques for the Dimensional Calibration of Standard Microscopic Particles. NBS Spec. Publ. 260-85 (In Press).

*Send order with remittance to Superintendent of Documents, U.S Government Printing Office, Washington, DC 20402. Remittance from foreign countries should include an additional onefourth of the purchase price for postage. Phone for current prices: 202-783-3238.

** May be ordered from National Technical Information Service (NTIS). Springfield, VA 22151. Phone: 703-487. 4650. 


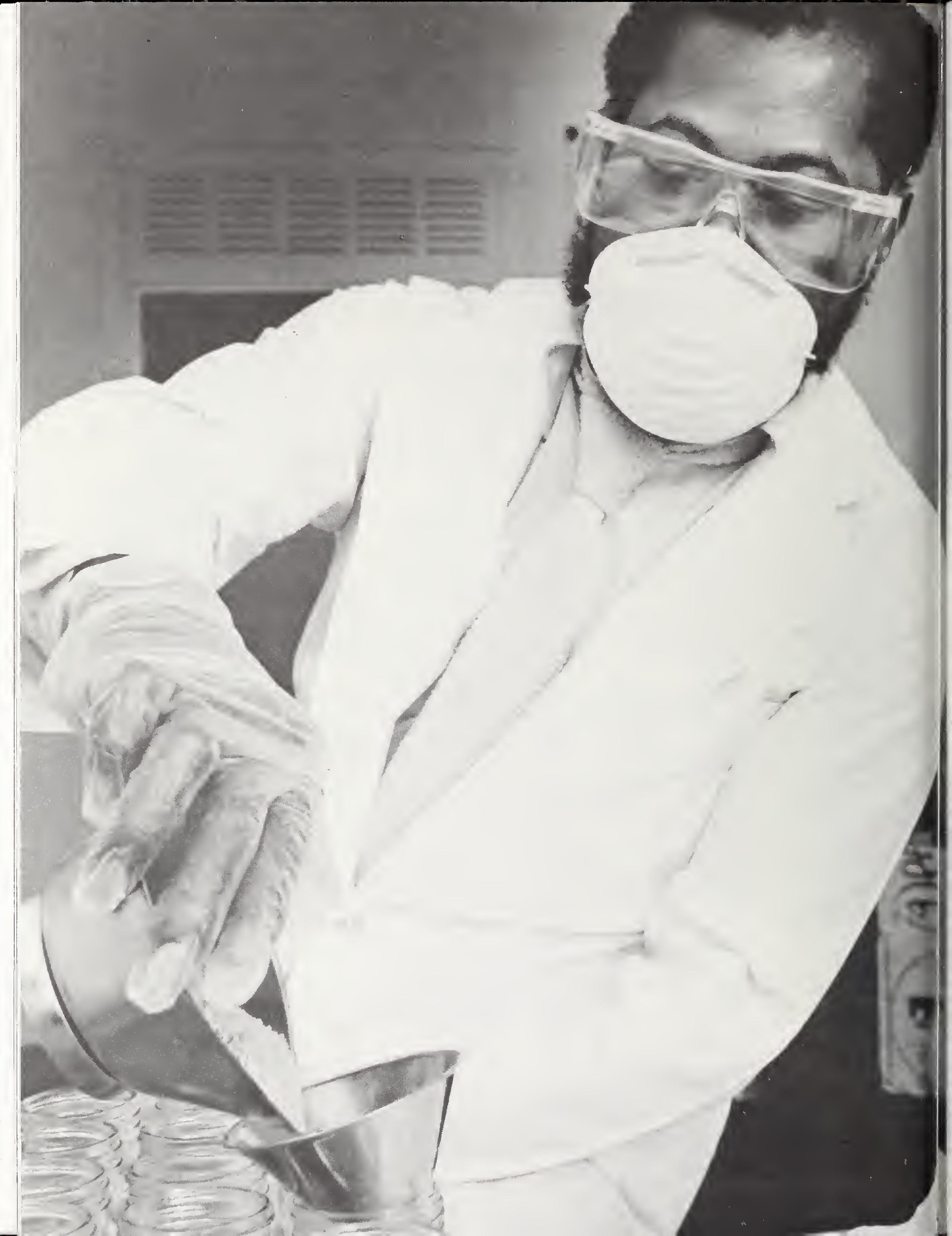




\section{Indexes}

\section{Category Index of Standards}

Abrasive Wear

Acidimetric

Acid Potassium Phthlate

Benzoic Acid

Boric Acid

Agricultural and Food Science

A mmonium Dihydrogen Phosphate (Fertilizer)

Bovine Liver

Brewers Yeast

Calcium Carbonate (Clinical)

Cholesterol (Clinical)

Citrus Leaves

Dextrose

Human Serum (Clinical)

Milk, Non-Fat Powdered

Oyster Tissue

Phosphate Rock (Fertilizer)

Pine Needles

Potassium Dihydrogen Phosphate (Fertilizer)

Potassium Nitrate (Fertilizer)

Rice Flour

Sucrose

Tomato Leaves

Trace Elements in Water

Tuna, Albacore

Wheat Flour

Wine, Stabilized

Air

Carbon Dioxide in

Carbon Monoxide in

Methane in

Methane-Propane in

Nitrogen Dioxide in

Propane in

Albumin

Bovine Serum (Powder)

Bovine Serum (7\% Solution)

Alloys

Casting

High

High-Temperature

Low

Nonferrous

Steelmaking

\section{Alloys (Chemical Composition)}

(also see individual metals)

Aluminum

Chip Form

Neutron Density Monitor Wire

Solid Form

Cobalt

Neutron Density Monitor Wire

Copper

Chip Form

Microprobe

Solid Form

Ferro (Steelmaking)

Gold

High-purity

Microprobe

High Temperature Solid Form

Iron

Chip Form

Microprobe

Solid Form

Lead

Chip Form

Solid Form

Magnesium

Chip Form

Molybdenum

Microprobe

Nickel

Chip Form

Oxides

Solid Form

Platinum

Doped, wire

High-purity, wire 
Selenium

$$
\text { Granular Form }
$$

Silver

Microprobe

Solder

Chip Form

Solid Form

Spelter

Stainless

Steelmaking

Tin

Chip Form

Titanium

Chip Form

Solid Form

Gas in

Tungsten

Microprobe

Zinc

Chip Form

High-purity

Solid Form

Zirconium

$$
\text { Chip Form }
$$

Solid Form

Alumina

Melting Point

Polycrystalline (Elasticity)

Reduction Grade

\section{Aluminum}

Al-Co Alloy (neutron density monitor wire)

Al-Si Alloy

"Benchmark"

Conductivity

Freezing Point

Magnetic Susceptibility

Metallo-organic Compound

Radioactivity

Residual Resistivity Ratio

Secondary Reference Point

Ultra Purity

Wrought Alloy

Americium

Radioactivity

Ammonium Dihydrogen Phosphate

Fertilizer
Analyzed Gases (Environmental)

Benzene Permeation Tube

Benzene in Nitrogen

Carbon Dioxide in Air

Carbon Dioxide in Nitrogen

Carbon Monoxide in Air

Carbon Monoxide in Nitrogen

Methane in Air

Methane-Propane in Air

Nitric Oxide in Nitrogen

Nitrogen Dioxide in Air

Nitrogen Dioxide Permeation Tube

Oxygen in Nitrogen

Propane in Air

Propane in Nitrogen

Propane in Nitrogen and Oxygen

Sulfur Dioxide in Nitrogen

Sulfur Dioxide Permeation Tube

Tetrachloroethylene in Nitrogen

\section{Analyzed Liquids and Solids}

(Environmental)

Estuarine Sediment

Halocarbons (in methanol) for Water Analysis

Lead in Reference Fuel

Mercury in Water

Petroleum Crude Oil

Polychlorinated Biphenyls in Oil

Polynuclear Aromatic Hydrocarbon Generator Columns

Powdered Lead Base Paint

Priority Pollutant Polynuclear Aromatic Hydrocarbons (in Acetonitrile)

River Sediment

Shale Oil

Sulfur in Coal

Sulfur in Distillate Fuel Oil

Sulfur in Residual Fuel Oil

Trace Elements in Coal

Trace Elements in Coal Fly Ash

Trace Elements in Fuel Oil

Trace Elements in Water

Trace Mercury in Coal

Urban Dust

Urban Particulate

Vanadium in Crude Oil

Vanadium and Nickel in Residual Fuel Oil

Angiotensin I (Human)

Antimony

Radioactivity

Arsenic

Trioxide (reductometric)

Asbestos

Chrysotile

Assay Standards

(See Specific Constituent)

Austenite

in Ferrite 
Barium

Metallo-organic Compound

101

73

55

71

71

70

59

61

55

93

\section{6}

Radioactivity

Basalt Rock

Basimetric

Tris(hydroxymethyl)aminomethane

\section{Bauxite}

Arkansas

Dominican

Jamaican

Surinam

\section{Benzene}

in Nitrogen

Permeation Device

Benzoic Acid

Acidimetric

Calorimetric

Microchemical

\section{Beryllium}

Bilirubin (Clinical)

Biological

Bovine Liver

Brewers Yeast

Citrus Leaves

Milk, Non-Fat Powdered

Oyster Tissue

Pine Needles

Rice Flour

Tomato Leaves

Tuna, Albacore

Wheat Flour

\section{Blast Furnace Irons}

Boron

Boric Acid

Boric Acid (Enriched)

Glass, High

Glass, Low

Botanical

(see Biological)

Bovine

Liver (Biological)

Serum Albumin (Powder)

Serum Albumin (7\% solution)

\section{Brass}

Aluminum

Cartridge

Free Cutting

Naval

Red

Sheet

\section{Bronze}

Commercial

Phosphor

Silicon on Filter Media
111

111

67

94

56

67

35

93

93

94

93

93

55,100

74

74

59

59

60

59

101

111

36

36

36

36

36

36

36

36

38

38

106

112

75

75

113

74

74

72

73
Butyl

Rubber

Cadmium

Metallo-organic Compound

Vapor Pressure

\section{Calcium}

Carbonate

Metallo-organic Compound

Molybdate (Steelmaking)

\section{Calorimetric}

Combustion

Enthalpy and Heat Capacity

Heat Source

Solution

Cane Sugar

Sucrose

Carbides

Tungsten

Carbon

Dioxide in Air

Dioxide in Nitrogen

Monoxide in Air

Monoxide in Nitrogen

Radioactivity

Rubber Compounding

Carbon Steels

Cast Iron, Chip Form

Ductile

Electrolytic

Gray

High Alloy White

Nodular

White

Cast Iron, Solid Form

White

Catalyst Package for Lubricant Oxidation

Centerline Drawings for Optical Character Recognition

Cements

Portland, Chemical Composition

Turbidimetric and Fineness

Ceramic Materials

Carbides

Glasses

Minerals

Refractories 


\begin{tabular}{|c|c|c|c|}
\hline 101 & Cesium & 57 & Iron Metal \\
\hline 101,103 & Radioactivity & 56 & Lead Nitrate \\
\hline 55 & Chemical & 56 & Liquid Filters for Spectrophotometry \\
\hline 55 & Primary & 56 & Lithium Carbonate \\
\hline 55 & Acid Potassium Phthalate & 56 & Magnesium Gluconate \\
\hline 55 & Arsenic Trioxide & 56 & D-Mannitol \\
\hline 55 & Benzoic Acid & 57 & 4-Nitrophenol \\
\hline 55 & Boric Acid & 56 & Potassium Chloride \\
\hline 55 & Dextrose & 57 & Quartz Cuvette for Spectrophotometry \\
\hline 55 & Potassium Chloride & 57 & Quinine Sulfate Dihydrate \\
\hline 55 & Potassium Dichromate & 57 & Rubidium Melting Point \\
\hline 55 & Sodium Oxalate & 56 & Sodium Chloride \\
\hline 55 & Strontium Cabonate & 56 & Sodium Pyruvate \\
\hline 55 & Sucrose & 57 & Stabilized Wine \\
\hline 55 & Tris(hydroxymethyl)aminomethane & 57 & Succinonitrile Freezing Point \\
\hline 55 & Uranium Oxide & 57 & Thermometer \\
\hline 44 & Intermediate Purity & 57 & Tripalmitin \\
\hline 44 & Selenium & 56 & Tris(hydroxymethyl)aminomethane \\
\hline & Chlorine & 56 & Tris(hydroxymethyl)aminomethane $\mathrm{HCl}$ \\
\hline 80 & Isotopic Reference & 56 & Urea \\
\hline 101 & Radioactivity & 56 & Uric Acid \\
\hline & Chromium & 56 & VMA \\
\hline 85 & Coating Thickness & & Coal \\
\hline 87 & on Brass & 61 & Mercury in \\
\hline 85 & on Steel & 65 & Sulfur in \\
\hline 35 & Ferro (Steelmaking) & 62 & Trace Elements in \\
\hline 67 & Metallo-organic Compound & 62 & Trace Elements in Fly Ash \\
\hline 80 & Nitrate (Isotopic) & 85 & Coating Thickness \\
\hline 105 & Radioactivity & 85 & Copper and Chromium on Steel \\
\hline 73 & Refractories & 87 & Gold on Glass Sealing Alloy \\
\hline 72 & Clays & 87 & Gold on Nickel \\
\hline 72 & Flint & 87 & Nickel and Chromium on Brass \\
\hline 72 & Plastic & 86 & Nickel and Steel \\
\hline 56 & Clinical Laboratory & & Cobalt \\
\hline 57 & Angiotensin I (Human) & 79 & Alloys \\
\hline 57 & Anticonvulsant Drug Level Assay & 67 & Metallo-organic Compounds \\
\hline 56 & Antiepilepsy Drug Level Assay & 101,105 & Radioactivity \\
\hline 56 & Bilirubin & 114 & Color \\
\hline 56 & Bovine Serum Albumin (Powder) & & Copper \\
\hline 56 & Bovine Serum Albumin ( $7 \%$ Solution) & 39,46 & Alloys \\
\hline 56 & Calcium Carbonate & 41 & "Benchmark", chip \\
\hline 56 & Cholesterol $=$ & 48 & "Benchmark", solid \\
\hline 57 & Clinical Laboratory Thermometer & 46 & Beryllium copper alloys \\
\hline 56 & Creatinine & & Brass \\
\hline 57 & Crystalline Potassium Dichromate & 46 & Aluminum \\
\hline 56 & Cortisol & 46,53 & Cartridge \\
\hline 57 & Gallium Melting Point & 46 & Free Cutting \\
\hline 56 & Glass Filters for Spectrophotometry & 46 & Naval \\
\hline 56 & D-Glucose & 46 & Red \\
\hline 56 & Human Serum & 39 & Sheet \\
\hline & & & Bronze \\
\hline & & 46 & Commercial \\
\hline & & 39 & Phosphor \\
\hline & & 39 & Silicon \\
\hline & & 85 & Coating Thickness \\
\hline & & 85 & on Steel \\
\hline & & 39 & Cupro-nickel, chip \\
\hline & & 46 & Cupro-nickel, solid \\
\hline & & 92 & Freezing Point \\
\hline & & 46 & Gilding Metal \\
\hline & & 94 & Heat Capacity \\
\hline & & 80 & Metal (Isotopic) \\
\hline & & 67 & Metallo-organic Compound \\
\hline
\end{tabular}




\begin{tabular}{|c|c|c|c|}
\hline 89 & Microhardness & 35 & Ferro-(Steelmaking Alloys) \\
\hline 54 & Microprobe (gold-copper wire) & 35 & Chromium \\
\hline 69 & Mill Heads & 35 & Niobium \\
\hline 69 & Mill Tails & 35 & Phosphorus \\
\hline 39 & Nickel Silver Alloy & 35 & Silicon \\
\hline 92 & Secondary Reference Point & 68 & Fertilizer \\
\hline 95 & Thermal Expansion & 68 & Ammonium Dihydrogen Phosphate \\
\hline 107 & Corrosion, Pitting or Crevice & 68 & Phosphate Rock \\
\hline 90 & Density & 68 & Potassium Dihydrogen Phosphate \\
\hline 90 & Borosilicate Glass & 68 & Potassium Nitrate \\
\hline 90 & Lead-Silica Glass & 96 & Filters \\
\hline 79 & Neutron Density Wire & 96 & Glass for Spectrophotometry \\
\hline 115 & Photographic & 96 & Liquid for Spectrophotometry \\
\hline 90 & Silicon & 66 & Filter Media \\
\hline 115 & Smoke Density, Flaming & 66 & Beryllium on \\
\hline 115 & Smoke Density, Non-flaming & 66 & Lead on \\
\hline 90 & Soda-Lime Glass & 66 & Metals on \\
\hline 90 & 2,2,4-trimeth ylpentane & 66 & Quartz on \\
\hline 115 & $X-$ ray & 66 & Sulfate and Nitrate on \\
\hline 55 & Dextrose (D-Glucose) & 80 & Fission Track Glasses \\
\hline 56 & Clinical & 115 & Flammability \\
\hline 100 & Optical Rotation & 115 & Smoke Density, Flaming \\
\hline 55 & Primary & 115 & Smoke Density, Nonflaming \\
\hline 55 & Reductometric Value & 115 & Surface (hardboard sheet) \\
\hline 118 & Differential Thermal Analysis & 66 & Fluorine in Urine \\
\hline 118 & Glass Transition & 69 & Fluorspar \\
\hline 118 & Magnetic Transition & 69 & Assay \\
\hline $\begin{array}{l}118 \\
118\end{array}$ & $\begin{array}{l}\text { Melting Point } \\
\text { Transition Point }\end{array}$ & 69 & Geological \\
\hline & Drug Level Assay & 62 & Fly Ash \\
\hline 57 & Anticonvulsant & 62 & Trace Elements in \\
\hline 56 & Antiepilepsy & 57 & Food Science \\
\hline 38 & Ductile Irons & & (See Agriculture and Food Science) \\
\hline & Dust & 66 & Freeze Dried Urine \\
\hline 64 & Urban/Organic & 66 & Fluorine \\
\hline 111 & Engineering Type & $\begin{array}{l}66 \\
66\end{array}$ & Mercury \\
\hline 59 & Environmental & 00 & $\begin{array}{l}\text { Multi-element } \\
\text { Freezing Point }\end{array}$ \\
\hline 59 & Analyzed Gases & $\begin{array}{l}92 \\
92\end{array}$ & $\begin{array}{l}\text { Freezing Point } \\
\text { Defining Fixed Points }\end{array}$ \\
\hline 61 & Analyzed Liquids and Solids & 92 & $\begin{array}{l}\text { Defining Fixed Points } \\
\text { Tin }\end{array}$ \\
\hline 64 & Organic Constituents & 92 & $\begin{array}{l}\text { Tin } \\
\text { Zinc }\end{array}$ \\
\hline 61 & Permeation Tubes & 92 & $\begin{array}{l}\text { Zinc } \\
\text { Secondary Reference Points }\end{array}$ \\
\hline 65 & Polychlorinated Biphenyls in Oil & 92 & $\begin{array}{l}\text { Secondary Reference Points } \\
\text { Aluminum }\end{array}$ \\
\hline 105 & Radioactivity & 92 & Aluminum \\
\hline 65 & Sulfur in Coal & 92 & Copper \\
\hline 89 & Elasticity & 92 & Lead \\
\hline 108 & Electrical Resistivity and Conductivity & 92 & Mercury \\
\hline 109 & Conductivity & 57 & Succinonitrile \\
\hline 109 & Glass & 92 & Tin \\
\hline 108 & $\begin{array}{l}\text { Glass } \\
\text { Metals }\end{array}$ & 92 & Zinc \\
\hline 109 & $\begin{array}{l}\text { Metals } \\
\text { Residual Resistivity Ratio }\end{array}$ & & \\
\hline 108 & $\begin{array}{l}\text { Residual Resistivity Ratio } \\
\text { Silicon }\end{array}$ & & \\
\hline & Europium & & \\
\hline 101,103 & Radioactivity & & \\
\hline 72 & Feldspars & & \\
\hline 72 & Soda & & \\
\hline 72 & Potash & & \\
\hline & Ferrite & & \\
\hline 106 & Austenite in & & \\
\hline 106 & Iron Carbide in & & \\
\hline
\end{tabular}


Fuel Oil

Sulfur in Residual

Sulfur in Distillate

Trace Elements in

Vanadium and Nickel in Residual

Fuels

n-Heptane

Isooctane

Lead in Reference

62

62,108

Reference

Gadolinium

Radioactivity

Gallium

Melting Point

Radioactivity

Gases, Analyzed

Benzene Permeation Tube

Benzene in Nitrogen

Carbon Dioxide in Air

Carbon Dioxide in Nitrogen

Carbon Monoxide in Air

Carbon Monoxide in Nitrogen

Methane in Air

Methane-Propane in Air

Nitric Oxide in Nitrogen

Nitrogen Dioxide in Air

Nitrogen Dioxide Permeation Tube

Oxygen in Nitrogen

Propane in Air

Propane in Nitrogen

Propane in Nitrogen and Oxygen

Sulfur Dioxide in Nitrogen

Sulfur Dioxide Permeation Tube

Tetrachloroethylene in Nitrogen

Gases in Metals

Certified for Hydrogen, Oxygen, and Nitrogen

Gasoline

Lead in

Fuel Ratings

Geological

(See Ores, Rocks, Minerals,

Refractories)

Basalt Rock

Clay

Fluorspar

Obsidian Rock
Glass

Density

Directional-Hemispherical

Reflectance

Electrical Resistivity

Fibers for Microanalysis

Filters for Spectrophotometry

Fission Track

Fluorescence Source

Refractive Index

Sand

Spheres for Sieve Calibration (sizing standard)

Stress Optical Coefficient

Thermal Conductivity

Thermal Expansion

Trace Elements in

Glasses (Chemical Composition)

High-Boron

Lead-Barium

Low-Boron

Opal

Soda-Lime

Container

Flat

Float

Sheet

Glasses (Physical Properties)

Chemical Resistance (Durability)

Electrical Properties

Glass Liquidus Temperature

Relative Stress Optical Coefficient

Viscosity

Viscosity Fixpoints

Glucose (Dextrose)

Clinical

Primary Chemical

Gold

Coating Thickness on Glass Sealing Alloy on Nickel

High-purity

Microprobe

Radioactivity

Thermocouple Material

Vapor Pressure

Hardness, Micro

Heat

Calorimetric

Freezing Points

Melting Points

Superconductive Fixed Points

Thermal Conductivity

Thermal Expansion

Thermocouple Materials

Vapor Pressure

Heat Source

Zirconium-barium-chromate

High Alloy Steels

Chip Form 


\begin{tabular}{|c|c|c|c|}
\hline 52 & High-Purity Metals & & Krypton \\
\hline 52 & Electrical Residual Resistivity Ratio & 103 & Radioactivity \\
\hline 52 & Gold, wire and rod & & Lead \\
\hline 52 & Platinum, wire & 42 & Alloys \\
\hline 52 & Zinc & 74 & Barium Glass \\
\hline 33 & High Temperature Alloys & 26,42 & Bearing Metal \\
\hline & Hydrogen & 66 & on Filter Media \\
\hline 51 & in Titanium & 92 & Freezing Point \\
\hline 101 & Radioactivity & 108 & Gasoline \\
\hline & Indium & 80 & Metal (Isotopic) \\
\hline 105,106 & Radioactivity & 80 & Equal Atom \\
\hline 66 & Industrial Hygiene & $\begin{array}{l}80 \\
80\end{array}$ & Natural \\
\hline 67 & Asbestos & $\begin{array}{l}80 \\
67\end{array}$ & Radiogenic \\
\hline 66 & Freeze Dried Urine & $\begin{array}{l}67 \\
56\end{array}$ & $\begin{array}{l}\text { Metallo-organic Compound } \\
\text { Nitrate (Clinical) }\end{array}$ \\
\hline 66 & Fluorine & 61 & Powdered Lead Base Paint \\
\hline 66 & Mercury & 105 & Radioactivity \\
\hline 66 & Multi-element & 92 & Secondary Reference Point \\
\hline 66 & Materials on Filter Media & 42,49 & Solder \\
\hline 66 & Beryllium & 80 & Spike (Assay and Isotopic) \\
\hline 66 & Lead & 62 & in Reference Fuel \\
\hline $\begin{array}{l}66 \\
66\end{array}$ & $\begin{array}{l}\text { Metals } \\
\text { Quartz }\end{array}$ & 114 & Light Sensitive \\
\hline $\begin{array}{l}00 \\
66\end{array}$ & Sulfate and Nitrate & 114 & Faded Paper Strips \\
\hline 66 & Respirable Quartz & 114 & Light Sensitive Papers \\
\hline 24 & Ingot Iron & 114 & Light Sensitive Plastic Chips \\
\hline 83 & Ion-Activity & 72 & Limestone \\
\hline 84 & Ion-selective Electrodes & 72 & Argillaceous \\
\hline 84 & $\mathrm{pD}$ & 72 & Dolomitic \\
\hline 83 & $\mathrm{pH}$ & $\begin{array}{l}116 \\
116\end{array}$ & $\begin{array}{l}\text { Linerboard } \\
\quad \text { for Tape Adhesion Testing }\end{array}$ \\
\hline & Iron & $\begin{array}{l}116 \\
85\end{array}$ & $\begin{array}{l}\text { for Tape Adhesion l esting } \\
\text { Linewidth Measurement }\end{array}$ \\
\hline 36 & Alloys & & $\begin{array}{l}\text { Linewidth Measurement } \\
\text { Liquids, Analyzed }\end{array}$ \\
\hline 36 & Chip Form & $\begin{array}{l}61 \\
56\end{array}$ & $\begin{array}{l}\text { Liquids, Analyzed } \\
\text { Carbonate (Clinical) }\end{array}$ \\
\hline 108 & Electrical Resistivity & 56 & Carbonate (Clinical) \\
\hline 24 & Ingot & 62 & \\
\hline 57 & Metal (Clinical) & 62 & Mercury in Water \\
\hline 67 & Metallo-organic Compound & 61 & Sulfur in Residual Fuel Oil \\
\hline 70 & Ores & 61 & Sulfur in Distillate Fuel Oil \\
\hline 104,105 & Radioactivity & 62 & Trace Elements in Fuel Oil \\
\hline 38 & Solid Form & 62 & Trace Elements in Water \\
\hline 94 & Thermal Conductivity & 62 & Vanadium in Crude Oil \\
\hline & Iodine & 61 & Vanadium and Nickel in Residual Fuel Oil \\
\hline 101,105 & Radioactivity & & Lithium \\
\hline 80 & Isotopic (Stable) & 69 & Ores \\
\hline 80 & Boric Acid, enriched & & Liver \\
\hline 80 & Boric Acid, natural & 105 & Human, Radioactivity \\
\hline 80 & Chromium Nitrate & 57 & Bovine, Biological \\
\hline 80 & Copper & & Low Alloy Steels \\
\hline 80 & Lead, equal atom $(206 / 208)$ & 20 & Chip Form \\
\hline 80 & Lead, Natural & 26 & Solid Form \\
\hline 80 & Lead, radiogenic, 92\% Lead.206 & & \\
\hline 80 & Lead-206, spike & & \\
\hline 80 & Magnesium & & \\
\hline 80 & Potassium Chloride & & - \\
\hline 80 & Rhenium & & \\
\hline 80 & Silicon & & \\
\hline 80 & Silver Nitrate & & \\
\hline 80 & Sodium Bromide & & \\
\hline 80 & Sodium Chloride & & \\
\hline 80 & Strontium Carbonate & & \\
\hline
\end{tabular}




\begin{tabular}{|c|c|}
\hline 106 & $\begin{array}{l}\text { Lubricant Oxidation } \\
\text { Catalyst Package for }\end{array}$ \\
\hline 105 & Lung \\
\hline 105 & Human, Radioactivity \\
\hline & Magnesium \\
\hline 42 & Alloy \\
\hline 56 & Gluconate (Clinical) \\
\hline 80 & Metal (Isotopic) \\
\hline 67 & Metallo-organic Compound \\
\hline 95 & Magnetic \\
\hline 95 & Moment \\
\hline 96 & Paramagnetic Resonance \\
\hline 95 & Susceptibility \\
\hline 112 & Tape (Computer Amplitude Reference) \\
\hline 84 & Magnification \\
\hline & Manganese \\
\hline 95 & Fluoride, Magnetic Susceptibility \\
\hline 67 & Metallo-organic Compound \\
\hline 101,103 & Radioactivity \\
\hline 32,51 & Maraging Steel \\
\hline 66 & Materials on Filter Media \\
\hline 66 & Beryllium \\
\hline 66 & Lead \\
\hline 66 & Metals \\
\hline 66 & Quartz \\
\hline 66 & Sulfate and Nitrate \\
\hline 84 & Mechanical and Metrology \\
\hline 85 & Linewidth Measurement \\
\hline 115 & Microscopy Resolution Test Chart \\
\hline 113 & Particle Size \\
\hline 84 & SEM Magnification \\
\hline 84 & SEM Performance \\
\hline & Mercury \\
\hline 92 & Freezing Point \\
\hline 105 & Radioactivity \\
\hline 92 & Secondary Reference Point \\
\hline 61 & Trace Element in Coal \\
\hline 66 & Trace Element in Urine \\
\hline 62 & Trace Element in Water \\
\hline 62 & in Water \\
\hline 93 & Melting Points \\
\hline 93 & Alumina \\
\hline 93 & Gallium \\
\hline 93 & Rubidium \\
\hline
\end{tabular}

\begin{tabular}{|c|c|}
\hline 67 & Metallo-Organic Compounds \\
\hline 67 & Aluminum \\
\hline 67 & Barium \\
\hline 67 & Cadmium \\
\hline 67 & Calcium \\
\hline 67 & Chromium \\
\hline 67 & Cobalt \\
\hline 67 & Copper \\
\hline 67 & Iron \\
\hline 67 & Lead \\
\hline 67 & Magnesium \\
\hline 67 & Manganese \\
\hline 67 & Nickel \\
\hline 67 & Phosphorus \\
\hline 67 & Potassium \\
\hline 67 & Silicon \\
\hline 67 & Silver \\
\hline 67 & Sodium \\
\hline 67 & Strontium \\
\hline 67 & Tin \\
\hline 67 & Vanadium \\
\hline 67 & Zinc \\
\hline 106 & Metallurgical \\
\hline 106 & Austinite in Ferrite \\
\hline \multirow[t]{4}{*}{106} & Iron Carbide in Ferrite, Spheroidized \\
\hline & Metals \\
\hline & Alloys (See Index Entry) \\
\hline & Elements (See Index Entry) \\
\hline 92 & Freezing Points \\
\hline 51 & Gas in \\
\hline 52 & High-purity \\
\hline 93 & Melting Points \\
\hline 53 & Microprobe \\
\hline 108 & Resistivity and Conductivity \\
\hline 94 & Thermal Conductivity \\
\hline 94 & Vapor Pressure \\
\hline 68 & Wear Metals in Oil \\
\hline & Metals in Oil \\
\hline 68 & Wear \\
\hline 66 & Metals on Filter Media \\
\hline 66 & Beryllium \\
\hline 66 & Cadmium \\
\hline 66 & Lead \\
\hline 66 & Manganese \\
\hline 66 & Zinc \\
\hline & Methane \\
\hline 59 & in Air \\
\hline 59 & $\begin{array}{l}\text { Methane-Propane } \\
\text { in Air }\end{array}$ \\
\hline 53 & Microanalytical \\
\hline 53 & Cartridge Brass \\
\hline 53 & $\mathrm{Fe} \cdot \mathrm{Cr} \cdot \mathrm{Ni}$ Alloy \\
\hline 53 & Glasses for Microchemical Analysis \\
\hline 54 & Gold-copper \\
\hline 54 & Gold-silver \\
\hline 54 & Iron-3.22\% Silicon \\
\hline 53 & Mineral Glasses for Microanalysis \\
\hline 54 & Tungsten-20\% Molybdenum \\
\hline
\end{tabular}




\begin{tabular}{|c|c|c|c|}
\hline 55 & Microchemical & & \\
\hline 55 & Acetanilide & & Molybdenum \\
\hline 55 & Anisic Acid & 49 & Alloy \\
\hline 55 & Benzoic Acid & 69 & Concentrate (ore) \\
\hline 56 & O-Bromobenzoic Acid & 94 & Heat Capacity \\
\hline 56 & m-Chlorobenzoic Acid & 54 & Microprobe \\
\hline 55 & Cystine & 105 & Radioactivity \\
\hline 56 & p.Fluorobenzoic Acid & 106 & Mössbauer \\
\hline 55 & Nicotinic Acid & 79 & Neutron Density Monitor Wire \\
\hline 56 & Urea & 79 & Cobalt in aluminum \\
\hline 115 & Microcopy Resolution Test Charts & & Nickel \\
\hline 89 & Microhardness & 42,49 & Alloys \\
\hline 89 & Bright Copper & 42 & Chip Form \\
\hline 89 & Bright Nickel & 49 & Solid Form \\
\hline & Microprobe & & Coating Thickness \\
\hline 53 & Cartridge Brass & 87 & on Brass \\
\hline 53 & Fe-Cr-Ni Alloy & 87 & on Gold \\
\hline 53 & Glasses for Microchemical Analysis & 86 & on Steel \\
\hline 54 & Gold-copper & 95 & Magnetic Moment \\
\hline 54 & Gold-silver & 67 & Metallo-organic Compound \\
\hline 54 & Iron-3.22\% Silicon & 89 & Microhardness \\
\hline 53 & Mineral Glasses for Microanalysis & 43 & Oxides \\
\hline 54 & Tungsgen-20\% Molybdenum & 101 & Radioactivity \\
\hline 57 & Milk, Non-Fat Powdered & & Niobium \\
\hline 72 & Minerals & 103 & Radioactivity \\
\hline & (See Rocks, and X-ray Diffraction) & & Nitric Oxide \\
\hline 72 & Clays & 59 & in Nitrogen \\
\hline 72 & Flint & & Nitrogen \\
\hline 72 & Plastic & 60 & Dioxide in Air \\
\hline 72 & Feldspar & 61 & Dioxide Permeation Tube \\
\hline 72 & Potash & & Nitrogen in \\
\hline 72 & Soda & 51 & Electrolytic Iron \\
\hline 72 & Glass Sand & 51 & Ingot Iron \\
\hline 72 & Limestone & 51 & $\begin{array}{l}\text { Ingot iron } \\
\text { Steel }\end{array}$ \\
\hline 72 & Argillaceous & 51 & Zirconium \\
\hline 72 & Dolomitic & 109 & $\begin{array}{l}\text { Zirconium } \\
\text { Nondestructive Evaluation }\end{array}$ \\
\hline 69,70 & Ores & 109 & $\begin{array}{l}\text { Nondestructive Evaluation } \\
\text { Penetrant Test Block }\end{array}$ \\
\hline 70 & Bauxite & 39 & $\begin{array}{l}\text { Penetrant Test Block } \\
\text { Nonferrous Alloys, Chip Form }\end{array}$ \\
\hline 69 & Copper & 39 & $\begin{array}{l}\text { Nonferrous Alloys, Chip Form } \\
\text { Aluminum-base }\end{array}$ \\
\hline 69 & Fluorspar & & \\
\hline 70 & Iron & 39 & Copper-base \\
\hline 69 & Lithium & 41 & Copper "Benchmark" \\
\hline 71 & Manganese & 42 & Lead-base \\
\hline 69 & Molybdenum & 42 & Magnesium-base \\
\hline 71 & Phosphate Rock & 42 & Nickel-base \\
\hline 71 & Rutile & 43 & Nickel Oxide \\
\hline 71 & Tungsten & 44 & Selenium \\
\hline 71 & Zinc & 43 & Trace Elements in Nickel-base Superalloy \\
\hline 98 & Mirrors & 44 & Tin-base \\
\hline 98 & · First Surface & 44 & Titanium-base \\
\hline 98 & Aluminum on Glass & 45 & Zinc-base \\
\hline 98 & Gold on Glass & 45 & Zirconium-base \\
\hline 98 & Second Surface & & \\
\hline 98 & Aluminum on Fused Quartz & & \\
\hline 90 & Molecular Weight & & \\
\hline 90 & Polyethylene & & \\
\hline 90 & Polystyrene & & \\
\hline
\end{tabular}




\section{Nonferrous Alloys, Solid Form}

Aluminum "Benchmark"

Copper-base

Copper "Benchmark"

Lead-base

Nickel-base

Titanium-base

Zinc-base

Zirconium-base

Nuclear Containers

Nuclear Materials

Fission Track Glass

Plutonium Assay

Plutonium Isotopic

Radiation Dosimetry

Neutron Density Monitor Wire

Uranium Assay

Uranium Isotopic

\section{Nutrition}

(See Agriculture and Food Science)

\section{Obsidian Rock}

Oil

Lubricant Oxidation

Petroleum Crude

Shale

Sulfur in Distillate Fuel

Sulfur in Residual Fuel

Trace Elements in

Vanadium in Crude

Vanadium and Nickel in Residual Fuel

Wear Metals in

\section{Optical Microscope Linewidth}

\section{Measurement}

\section{Optical}

Directional-Hemispherical Reflectance

Optical Rotation

Reflectance

Refractive Index

Spectrophotometric

Specular Spectral Reflectance

Ores

Alumina

Bauxite

Copper

Fluorspar

Iron

Lithium

Manganese

Molybdenum

Phosphate Rock

Rutile

Tungsten

Zinc

\section{Oxalic Acid \\ Radioactivity \\ Oxidimetric}

Potassium Dichromate

Oxides

Iron

Nitrogen

Titanium

Uranium

Zinc

Oxygen

Ingot Iron

in Nitrogen

Steel

Titanium

Zirconium

Palladium

Magnetic Susceptibility

\section{Paint}

Lead-base

Paper

Faded Strips

Light Sensitive

Particle Size

Glass Spheres

Polystyrene Spheres

\section{Particulate} Urban

Permeation Tubes

Benzene

Nitrogen Dioxide

Sulfur Dioxide

$\mathrm{pD}$

Disodium Hydrogen Phosphate

Potassium Dihydrogen Phosphate

Sodium Bicarbonate

Sodium Carbonate

$\mathrm{pH}$

Disodium Hydrogen Phosphate Potassium Dihydrogen Phosphate Potassium Hydrogen Phthalate Potassium Hydrogen Tartrate

Potassium Tetroxalate

Sodium Bicarbonate

Sodium Carbonate

Sodium Tetraborate Decahydrate (Borax)

Tris(hydroxymethyl)aminomethane

Tris(hydroxymethyl)aminomethane $\mathrm{HCl}$

Phosphate

(See $\mathrm{pD}$ and $\mathrm{pH}$ Standards)

Ammonium Dihydrogen

Potassium Dihydrogen

Rock

Phosphorus

Ferro (Steelmaking alloy)

Metallo-organic Compound Radioactivity

Photographic Microcopy Resolution Test Chart Step Tablets 


\begin{tabular}{|c|c|c|c|}
\hline 107 & Pitting or Crevice Corrosion & 107 & Powder Diffraction \\
\hline & Plastic & 107 & Alpha-alumina \\
\hline 90 & (See Polymer) & 107 & Cerium Oxide \\
\hline 114 & Light Sensitive Chips & 107 & Chromium Oxide \\
\hline 108 & Polyester Film for Oxygen Transmission & 107 & Mica \\
\hline 19 & Plain Carbon Steels & 107 & Rutile \\
\hline & Platinum & 107 & Silicon \\
\hline 52 & Doped, wire & 107 & Zinc Oxide \\
\hline 52 & High-purity, wire & 55 & Primary Chemicals \\
\hline 95 & Magnetic Susceptibility & 55 & Acid Potassium Phthalate \\
\hline 95 & Thermocouple Material & 55 & Arsenic Trioxide \\
\hline & Plutonium & 55 & Benzoic Acid \\
\hline 77 & Assay & 55 & Boric Acid \\
\hline 77 & Metal & 55 & Dextrose \\
\hline 77 & Standard Matrix & 55 & Plutonium Metal \\
\hline 77 & Isotopic & 55 & Potassium Chloride \\
\hline 77 & Spike & 55 & Potassium Dichromate \\
\hline 77 & Sulfate Tetrahydrate & 55 & Sodium Oxalate \\
\hline 101,102 & Radioactivity & 55 & Strontium Carbonate \\
\hline 55 & Polarimetric & $\begin{array}{l}55 \\
55\end{array}$ & Sucrose \\
\hline 55 & Dextrose & $\begin{array}{l}55 \\
55\end{array}$ & $\begin{array}{l}\text { Tris(hydroxymethyl)aminomethane } \\
\text { Uranium Metal }\end{array}$ \\
\hline 55 & Sucrose & 55 & Uranium Oxide \\
\hline 59 & $\begin{array}{l}\text { Pollution } \\
\quad \text { (See Environmental) }\end{array}$ & 59 & $\begin{array}{l}\text { Propane } \\
\text { in Air }\end{array}$ \\
\hline 108 & Polyester Plastic Film & 60 & in Nitrogen \\
\hline & Polyethylene & 60 & in Nitrogen and Oxygen \\
\hline 94 & Calorimetric & & Quartz \\
\hline 90 & Polymer & 66 & Alpha-for Solution Calorimetry \\
\hline & Polymer & 96 & Cuvette for Spectrophotometry \\
\hline 90 & Gas Transmission & 66 & on Filter Media \\
\hline 90 & Molecular Weight & 97 & Metal-on (Spectrophotometry) \\
\hline 90 & Polyethylene & 66 & Respirable \\
\hline 90 & Polystyrene & & Quinine \\
\hline & Polystyrene & 96 & Sulfate Dihydrate (spectrofluorometric) \\
\hline 94 & Calorimetric & 100 & Radioactivity \\
\hline 90 & Polymer & 101 & Alpha-particle, Beta-particle, Gamma-ray, \\
\hline 75 & Portland Cement & & and Electron-capture Solutions \\
\hline 75 & Composition & 102 & Alpha-particle Point Source \\
\hline 113 & Turbidimetric and Fineness & 105 & Environmental \\
\hline & Potassium & 103 & Gamma-ray and X-ray Point Source \\
\hline 55 & Acid Phthalate (Acidimetric) & 104 & Gamma-ray Solution \\
\hline & Chloride & 103 & Gaseous \\
\hline 55 & Assay Isotopic & 104 & Low-energy Photon \\
\hline 56 & Clinical & 102 & Radiocarbon Dating and Ground Water Studies \\
\hline 93 & Solution Calorimetry & 102 & Contemporary Standard for Carbon-14 Studies \\
\hline & Dichromate & 102 & Low-level Tritiated-water \\
\hline 55 & Clinical & 105 & Radiopharmaceutical \\
\hline 55 & Oxidimetric & 104 & Radium-226 Solution \\
\hline 96 & Spectrophotometric & 106 & Special Packaging \\
\hline 68 & Dihydrogen Phosphate (Fertilizer) & 79 & Radiation Dosimetry \\
\hline 76 & Feldspar, Trace Elements in & 79 & Neutron Density Monitor Wire \\
\hline 97 & Iodide (Spectrophotometric) & 79 & Vanadium in Crude Oil \\
\hline 83 & Ion Activity & & \\
\hline 84 & Ion Selective Electrodes & & \\
\hline 84 & $\mathrm{pD}$ & & \\
\hline 83 & $\mathrm{pH}$ & & \\
\hline 68 & Nitrate (Fertilizer) & & \\
\hline 67 & Metallo-organic Compound & & \\
\hline
\end{tabular}


Radium

Radioactivity

Reductometric

Arsenic Trioxide

Dextrose

Sodium Oxalate

Reference Fuel

n-Heptane

Isooctane

Reference Magnetic Tapes

Reflectance

Directional-Hemispherical

Refractive Index

Specular Spectral

Reflection Step Tablet

Refractive Index

Glass

Silicone Liquids

2,2,4-Trimethylpentane

Toluene

Refractory Materials

(See Rocks, Minerals and Refractories)

Burnt

Chrome

Silica

Research Materials

Albacore Tuna

Aluminum (Ultra Purity)

Copper Heat Capacity

Glass for Microanalysis

River Sediment

Resistivity

Electrical

Silicon

Rhenium

Isotopic and Assay
Rheology

Rocks, Minerals, and Refractories

Basalt

Burnt Refractory

Chrome Refractory

Clay

Feldspar

Glass Sand

Limestone

Obsidian Rock

Phosphate Rock

Silica Refractory

Titanium Dioxide

Rubber

Butyl

Butyl (Low Viscosity)

Styrene-butadiene 1500

Rubber Compounding Materials

Benzothiazyldisulfide

Channel Black

Gas Furnace Black

Mercaptobenzothiazole

N-tertiary-Butyl-

benzothiazolesulfidenamide

Oil Furnace Black

Stearic Acid

Sulfur

Zinc Oxide

Rubidium

Isotopic

Melting Point

Ruby

Paramagnetic Resonance

Rutile Ore

Sapphire

Enthalpy and Heat Capacity

Scanning Electron Microscope

Magnification

Performance

Secondary Standard Chemicals

(See Primary Standard Chemicals)

Sediment

Estaurine

$\begin{array}{ll}62 & \text { River (Environmental) } \\ 105,117 & \text { River (Radioactivity) }\end{array}$

$\begin{array}{cl}44 & \text { Alloy } \\ 44 & \text { Metal } \\ 105 & \text { Radioactivity }\end{array}$ 
Silica

Thermal Expansion

Silicon

Electrical Resistivity

Ferro (Steelmaking Alloy)

Isotopic and Assay

Metallo-organic Compound

Refractive Index

$X$-ray Diffraction

Silicone Liquid

Refractive Index

Silver

Alloys

Nitrate (Isotopic)

Metallo-organic Compound

Thermocouple Material

Vapor Pressure

Sizing

Particle Size

Calibrated Glass Spheres

Polystyrene Spheres

Turbidimetric and Fineness (Cement)

Smoke Density Chamber

Soda-Lime Glass

Container

Flat

Float

Sheet

Sodium

Bromide (Isotopic)

Chloride

Clinical

Isotopic

Ion-activity

Ion-selective Electrodes

$\mathrm{pD}$

$\mathrm{pH}$

Oxalate (Reductometric Value)

Pyruvate

Soil

(See Sediment)

62,117 Composition

105

105

42,49

42,49

42

49
61

62

61

62

62

61

62

62

118

119

119

118

32

96

96

96

96

96

96

97

96

97

96

98

98

98

22

108

30

94

35

35

35

35

35

35

35

35
Solids, Analyzed

Estuarine Sediment

Powdered Lead Base Paint

Trace Elements in Coal

Trace Elements in Coal Fly Ash

Trace Mercury in Coal

River Sediment

Urban Particulate

Special Reference Materials

Calibration of Surface Area of

Powders by BET

Calibration of Thermometry

(Melting Points)

Differential Thermal Analysis

Specialty Steels

Spectrofluorometric Standards

Quinine Sulfate Dihydrate

Spectrophotometric Standards

Didymium-oxide Glass Filters

Glass Filters

Liquid Filters

Metal on Quartz Filters

Potassium Dichromate

Potassium Iodide (Stray Light)

Quartz Cuvette

Specular Spectral Reflectance

First Surface Mirror

Second Surface Mirror

Stainless Steel

Chip Form

Electrical Resistivity

Solid Form

Steel

Thermal Conductivity

Steelmaking Alloys

Calcium Molybdate

Ferrochromium

Ferromanganese

Ferroniobium

Ferrophosphorus

Ferrosilicon

Silicon, Refined 


\section{Steels, Cast}

Steels, Chip Form

High Alloy

Low Alloy

Plain Carbon

Stainless

Tool

Steels, Gas In

Steels, Granular Form

Steels, Solid Form

Cast

High-temperature

Ingot Iron

Ingot Iron, Special

Low Alloy

Low Alloy, Special

Maraging Oxygen

Specialty

Stainless

Tool

Step Tablets

Photographic

$\mathrm{X}$-ray

Strontium

Carbonate (Assay and Isotopic)

Metallo-organic

101,106 Radioactivity

Sucrose

Optical Rotation

Primary Chemical

\section{Sulfur}

Dioxide in Nitrogen

Dioxide Permeation Tubes

in Coal

in Residual Fuel Oils

Rubber Compounding Materials
Reflection
Superconductive Thermometric Fixed

\section{Point Devices}

Surface Area

Alumina

Carbon Black

Silica

Surface Flammability

Tape Adhesion Testing Linerboard

Tape, Magnetic Cart ridge

Cassette

Disk

Reel

Technetium Radioactivity

Temperature (See Heat Standards)

Calorimetric

Differential Thermal Analysis

Freezing Point

Heat Source

Melting Point

Superconductive Thermometric Fixed Point Device

Thallium

Radioactivity

Thermal Conductivity

Thermal Expansion

Thermocouple Materials

Thermometers

Thorium

Radioactvity

Tin

Alloy

Chip Form

Freezing Point

Metallo-organic Compound

Radioactivity

Secondary Reference Point

Titanium

Chip Form

Dioxide

Gases in

Solid Form

Toluene

Refractive Index 


\section{Tool Steels}

Chip Form

Solid Form

Trace Elements

Biological Matrices

Environmental

Fuels

in Glass

Metallo-organic Compound

Potassium Feldspar

\section{2,2,4-Trimethylpentane}

Calorimetric

Density

Refractive Index

Tripalmitin

Tuna

Albacore

Tungsten

Alloy

Carbide

Concentrate

Electrical Resistivity

Enthalpy and Heat Capacity

Microprobe

Thermal Conductivity

Thermal Expansion
Turbidimetric and Fineness

\section{Uranium}

Assay

Metal

Oxide

Spike

Isotopic

Oxide

Depleted

Enriched

Primary

Urea

Calorimetric

Clinical

Microchemical

Urine

Fluorine in

Mercury in

Trace Elements in

Vanadium

and Nickel in Residual Fuel Oil

in Crude Oil

Metallo-organic Compound

Radioactivity

Vapor Pressure

Viscosity

Glass

Water
Trace Elements in
Water Analysis

Halocarbons (in methanol)

Trace Elements

Wear Metals in Oil

White Cast Irons

Wine

Stabilized

Working Standard Chemicals

(See Primary Standard Chemicals)

Xenon

103,106 Radioactivity

115 X-Ray and Photographic

115

115

115

115

107

107

107

107

115

107

106

Microcopy Resolution Test Chart

Photographic Film Step Tablet

Reflection Step Tablet

X-Ray Film Step Tablet

X-Ray Diffraction

Powder Diffraction Intensity

Powder Diffraction (Mica)

Silicon Powder

X-Ray Film Step Tablet

X-Ray Fluorescent Emission Target

Ytterbium

Radioactivity

Zinc

Alloy

Chip Form

Concentrate

Freezing Point

High Purity

Metallo-organic Compound

Oxide

Secondary Reference Point

Solid Form

Spelter (Modified)

Zirconium

45,51 Alloy

93 Barium Chromate (Calorimetric)

45 Chip Form

51 Gases in

51 Solid Form 


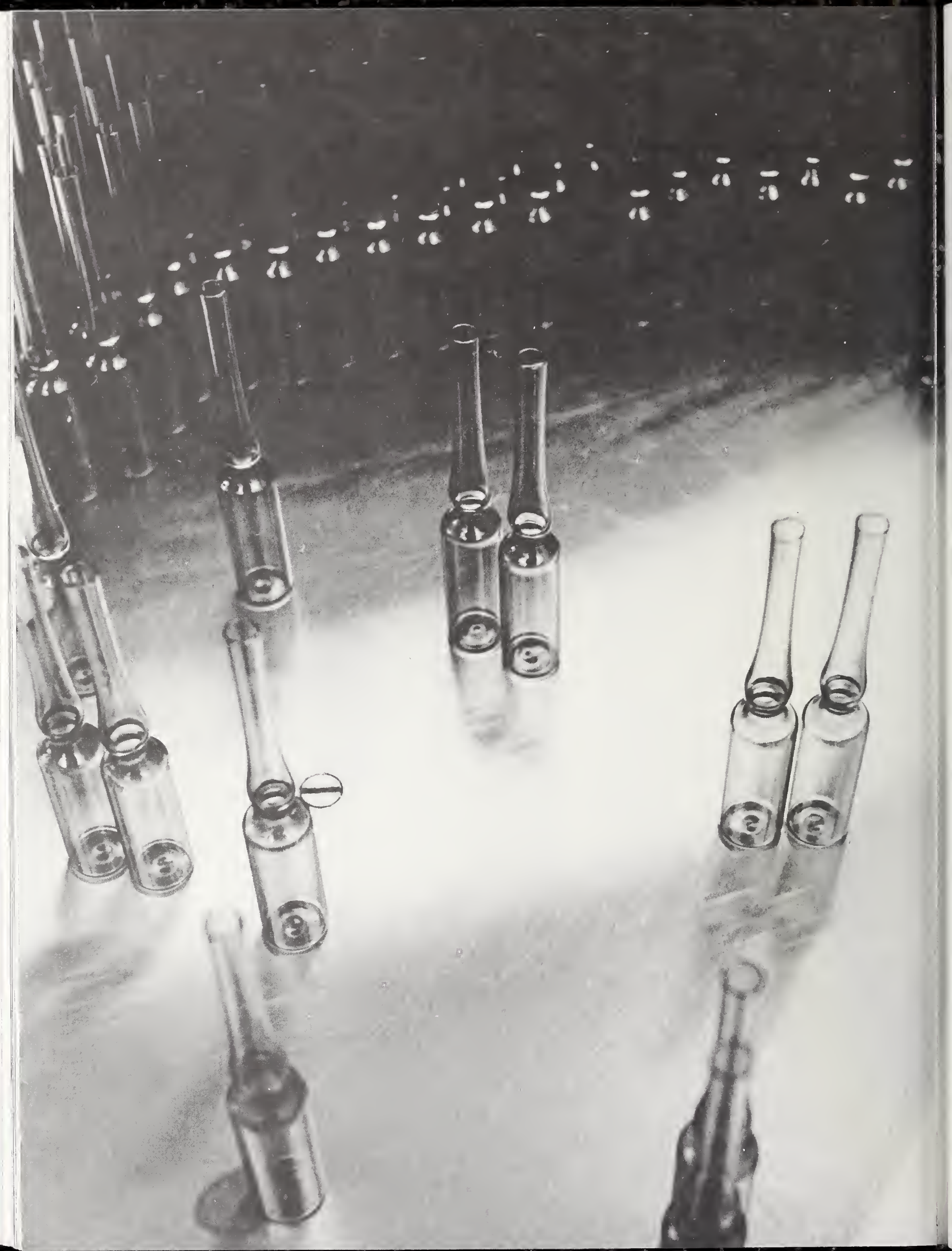




\section{Alphabeticall Index by Standard Reference Material Name}

\begin{tabular}{|c|c|c|c|c|c|}
\hline Page & Name & SRM & Page & Name & SRM \\
\hline 55 & Acetanilide & $141 \mathrm{c}$ & 92 & Aluminum, Freezing Point Standard & $44 f$ \\
\hline 19 & Acid Open-Hearth Steel, $0.2 \%$ Carbon & $19 \mathrm{G}$ & 95 & Aluminum, Magnetic Gram & 763 \\
\hline 55 & Acid Potassium Phthalate & $84 \mathbf{j}$ & & Susceptibility & \\
\hline 19 & AISI 1045 Steel & $20 \mathrm{~g}$ & 93 & Aluminum Oxide, Melting Point & 742 \\
\hline 20 & AISI 4340 Steel & 361 & 116 & Aluminum Rod Ultra Purity & RM $1 \mathrm{R}$ \\
\hline 26 & AISI 4340 Steel & $1261 \mathrm{a}$ & 101 & Aluminum-26 Radioactivity Standard & 4229 \\
\hline 20 & AISI 94B17 Steel (Modified) & 362 & 102 & Americium-241 Alpha-Particle & $4904 \mathrm{~F}$ \\
\hline 26 & AISI 94B17 Steel (Modified) & $1262 \mathrm{a}$ & & Standard & \\
\hline 117 & Albacore Tuna & RM 50 & 103 & Americium-241 Gamma-ray Standard & 4213 \\
\hline 88 & Alkali Lead Silicate Glass & 712 & 68 & Ammonium Dihydrogen Phosphate & 194 \\
\hline 66 & Alpha Quartz & 1878 & 57 & Angiotensin I (Human) & 998 \\
\hline 71 & Alumina (Reduction Grade) & 699 & 55 & Anisic Acid & 142 \\
\hline 88 & Alumina Silicate Glass & 714 & 57 & Anticonvulsant Drug Level Assay & 1599 \\
\hline 88 & Aluminosilicate Glass & 715 & & Standard & \\
\hline 39 & Aluminum Alloy & $85 \mathrm{~B}$ & 56 & Antiepilepsy Drug Level Assay & 900 \\
\hline 39 & Aluminum Alloy 6011 (Modified) & 858 & & Standard & \\
\hline 45 & Aluminum Alloy 6011 (Modified) & 1258 & 103 & Antimony-125-Tellurium-125m, & 4275B \\
\hline 39 & Aluminum Alloy 7075 & 859 & & Europium-154, Europium-155 Mixed- & \\
\hline 45 & Aluminum Alloy 7075 & 1259 & & Radionuclide Point-Source Standard & \\
\hline 109 & $\begin{array}{l}\text { Aluminum Block, Eddy Current } \\
\text { Conductivity }\end{array}$ & 1860 & 101 & $\begin{array}{l}\text { Antimony-125-Tellurium-125m, } \\
\text { Europium-154, Europium-155 Mixed- }\end{array}$ & $4276 \mathrm{~B}$ \\
\hline 109 & $\begin{array}{l}\text { Aluminum Block, Eddy Current } \\
\text { Conductivity }\end{array}$ & 1861 & 25 & $\begin{array}{l}\text { Radionuclide Solution Standard } \\
\text { A.O.H., 0.4C Spectrographic Steel }\end{array}$ & 413 \\
\hline 109 & $\begin{array}{l}\text { Aluminum Block, Eddy Current } \\
\text { Conductivity }\end{array}$ & 1862 & 72 & $\begin{array}{l}\text { Standard } \\
\text { Argillaceous Limestone }\end{array}$ & $1 \mathrm{C}$ \\
\hline 46 & $\begin{array}{l}\text { Aluminum Block, Eddy Current } \\
\text { Conductivity }\end{array}$ & 1863 & 55 & $\begin{array}{l}\text { Arsenic Trioxide Reductometric } \\
\text { Standard }\end{array}$ & $83 \mathrm{~d}$ \\
\hline 46 & Aluminum Brass Standard for & 1118 & 55,80 & Assay-Isotopic Standard for Potassium & 985 \\
\hline & Optical Emission and X-ray & & 80 & Assay-Isotopic Standard for Rhenium & 989 \\
\hline & Spectroscopic Analysis & & 80 & Assay-Isotopic Standard for Silicon & 990 \\
\hline 46 & Aluminum Brass Standard for & C1118 & 55,80 & Assay-Isotopic Standard for Strontium & 987 \\
\hline & Optical Emission and X-ray & & 106 & $2 \%$ Austenite in Ferrite & 488 \\
\hline & Spectroscopic Analysis & & 106 & $5 \%$ Austenite in Ferrite & $485 a$ \\
\hline 46 & Aluminum Brass Standard for & 1119 & 106 & $15 \%$ Austenite in Ferrite & 486 \\
\hline & $\begin{array}{l}\text { Optical Emission and X-ray } \\
\text { Spectroscopic Analysis }\end{array}$ & & 106 & $30 \%$ Austenite in Ferrite & 487 \\
\hline 46 & $\begin{array}{l}\text { Aluminum Brass Standard for } \\
\text { Optical Emission and X-ray } \\
\text { Spectroscopic Analysis }\end{array}$ & C1119 & & & \\
\hline 39 & Aluminum Casting Alloy 356 & 855 & & & \\
\hline 39 & Aluminum Casting Alloy 380 & 856 & & & \\
\hline 116 & Aluminum Cube Ultra Purity & RM 1C & & & \\
\hline 67 & Aluminum 2-Ethylhexanoate & $1075 \mathrm{a}$ & & & \\
\hline
\end{tabular}




\begin{tabular}{|c|c|c|c|c|c|}
\hline Page & Name & SRM & Page & Name & SRM \\
\hline 94,108 & $\begin{array}{l}\text { Austenitic Stainless Steel, Thermal } \\
\text { Conductivity and Electrical } \\
\text { Resistivity }\end{array}$ & 1460 & $\begin{array}{l}66 \\
19\end{array}$ & $\begin{array}{l}\text { Beryllium on Filter Media } \\
\text { Bessemer Steel (Simulated) } \\
0.1 \% \text { Carbon }\end{array}$ & $\begin{array}{l}2675 \\
8 \mathrm{j}\end{array}$ \\
\hline 94,108 & $\begin{array}{l}\text { Austenitic Stainless Steel, Thermal } \\
\text { Conductivity and Electrical } \\
\text { Resistivity }\end{array}$ & 1461 & $\begin{array}{l}56 \\
67\end{array}$ & $\begin{array}{l}\text { Bilirubin } \\
\text { Bis(1-phenyl-1, 3-butanediono) } \\
\text { copper (II) }\end{array}$ & $\begin{array}{l}916 \\
1080 \mathrm{a}\end{array}$ \\
\hline 94,108 & $\begin{array}{l}\text { Austenitic Stainless Steel, Thermal } \\
\text { Conductivity and Electrical }\end{array}$ & 1462 & 67 & $\begin{array}{l}\text { Bis(1-phenyl-1, 3-butanediono) } \\
\text { oxovanadium (IV) }\end{array}$ & $1052 b$ \\
\hline 88 & $\begin{array}{l}\text { Resistivity } \\
\text { Barium Crown Glass }\end{array}$ & 713 & 98 & $\begin{array}{l}\text { Black Porcelain Enamel for Directional } \\
\text { Hemispherical Reflectance }\end{array}$ & 2021 \\
\hline 67 & Barium Cyclohexanebutyrate & $1051 b$ & 98 & Black Porcelain Enamel for Directional & 2022 \\
\hline 103 & $\begin{array}{l}\text { Barrium-133 Radioactivity Point-Source } \\
\text { Standard } \\
\text { Barium-133 Radioactivity Standard }\end{array}$ & $4241 B$ & 38 & $\begin{array}{l}\text { Hemispherical Reflectance } \\
\text { Blast Furnace Iron Standard } \\
\text { (Chill Cast White) }\end{array}$ & $1143 a$ \\
\hline $\begin{array}{l}72 \\
68\end{array}$ & $\begin{array}{l}\text { Basalt Rock } \\
\text { Base Oil }\end{array}$ & $\begin{array}{l}688 \\
1083\end{array}$ & 38 & $\begin{array}{l}\text { Blast Furnace Iron Standard } \\
\text { (Chill Cast White) }\end{array}$ & $1144 a$ \\
\hline 25 & $\begin{array}{l}\text { Basic Electric Spectrographic Steel } \\
\text { Standard }\end{array}$ & $404 a$ & 25 & $\begin{array}{l}\text { B.O.H., 0.4C Spectrographic Steel } \\
\text { Standard }\end{array}$ & $417 \mathrm{a}$ \\
\hline 19 & Basic Open-Hearth Steel, $0.1 \%$ Carbon & $15 \mathrm{~g}$ & 55,80 & Boric Acid & 951 \\
\hline $\begin{array}{l}19 \\
26\end{array}$ & $\begin{array}{l}\text { Basic Open-Hearth Steel, 0.1\% Carbon } \\
\text { Basic Open-Hearth Steel, } 0.1 \% \text { Carbon }\end{array}$ & $\begin{array}{l}335 \\
1228\end{array}$ & 108 & $\begin{array}{l}\text { Boron-Doped Silicon Slices for } \\
\text { Resistivity Measurements }\end{array}$ & 1521 \\
\hline 19 & Basic Open-Hearth Steel, $0.2 \%$ Carbon & $11 \mathrm{~h}$ & 74 & Borosilicate Glass & $93 a$ \\
\hline 19 & Basic Open-Hearth Steel, $0.4 \%$ Carbon & $12 \mathrm{H}$ & 87 & Borosilicate Glass & 623 \\
\hline 19 & Basic Open-Hearth Steel, 0.5\% Carbon & $152 \mathrm{~A}$ & 88 & Borosilicate Glass & 717 \\
\hline 19 & Basic Open-Hearth Steel, 0.8\% Carbon & $14 f$ & 90 & Borosilicate Glass & 1825 \\
\hline 26 & $\begin{array}{l}\text { Basic Open-Hearth Steel, 1\% Carbon } \\
\text { (Disk) }\end{array}$ & 1227 & $\begin{array}{l}95 \\
57\end{array}$ & $\begin{array}{l}\text { Borosilicate Glass, Thermal Expansion } \\
\text { Bovine Liver }\end{array}$ & $\begin{array}{l}731 \\
1577 a\end{array}$ \\
\hline 19 & Basic Open-Hearth Steel, $1.1 \%$ Carbon & $16 f$ & 56 & Bovine Serum Albumin & 926 \\
\hline 19 & Basic Open-Hearth Steel, $1.1 \%$ Carbon & 337 & 56 & Bovine Serum Albumin ( $7 \%$ Solution) & 927 \\
\hline 19 & 0.4C Basic Oxygen Furnace Steel & 178 & 90 & Branched Polyethylene & 1476 \\
\hline 70 & Bauxite (Arkansas) & $69 b$ & 57 & Brewers Yeast & 1569 \\
\hline 71 & Bauxite (Dominican) & 697 & 89 & Bright Copper Microhardness & 1894 \\
\hline 71 & Bauxite (Jamaican) & 698 & & Standard & \\
\hline 70 & Bauxite (Surinam) & 696 & 89 & Bright Nickel Microhardness Standard & 1895 \\
\hline 59 & Benzene in Nitrogen & 1805 & 56 & Bromobenzoic Acid & 2142 \\
\hline 59 & Benzene in Nitrogen & 1806 & 72 & Burnt Refractory & $76 a$ \\
\hline 61 & Benzene Permeation Device & 1911 & 72 & Burnt Refractory & $77 \mathrm{a}$ \\
\hline 55 & Benzoic Acid & $140 b$ & 72 & Burnt Refractory & $78 \mathrm{a}$ \\
\hline 55 & Benzoic Acid & $350 \mathrm{a}$ & 67 & Cadmium Cyclohexanebutyrate & $1053 a$ \\
\hline 93 & Benzoic Acid Calorimetric Standard & $39 \mathrm{i}$ & 94 & Cadmium, Vapor Pressure & 746 \\
\hline 111 & $\begin{array}{l}\text { Benzothiazyl Disulfide Rubber } \\
\text { Compound }\end{array}$ & $373 \mathrm{f}$ & $\begin{array}{l}56 \\
67\end{array}$ & $\begin{array}{l}\text { Calcium Carbonate } \\
\text { Calcium 2-Ethylhexanoate }\end{array}$ & 915 \\
\hline 46 & Beryllium-Copper Standard & 1122 & 26 & Calcium in Low-Alloy (Silicon) Steel & 1254 \\
\hline 46 & Beryllium-Copper Standard & C1122 & 35 & Calcium Molybdate & 71 \\
\hline 46 & Beryllium-Copper Standard & C1123 & 113 & Calibrated Glass Beads & 1004 \\
\hline & & & 113 & Calibrated Glass Beads & $1017 \mathrm{a}$ \\
\hline & & & 113 & Calibrated Glass Beads & $1018 \mathrm{a}$ \\
\hline & & & 113 & Calibrated Glass Spheres & $1003 \mathrm{a}$ \\
\hline & & & 59 & Carbon Dioxide in Air & 1670 \\
\hline & & & 59 & Carbon Dioxide in Air & 1671 \\
\hline & & & 59 & Carbon Dioxide in Air & 1672 \\
\hline & & & 59 & Carbon Dioxide in Nitrogen & $1674 \mathrm{~b}$ \\
\hline & & & 59 & Carbon Dioxide in Nitrogen & $1675 b$ \\
\hline & & & 60 & $\begin{array}{l}\text { Carbon Dioxide in Nitrogen } \\
\text { (Combustion Efficiency Gas Standard) }\end{array}$ & $2619 a$ \\
\hline & & & 60 & $\begin{array}{l}\text { Carbon Dioxide in Nitrogen } \\
\text { (Combustion Efficiency Gas Standard) }\end{array}$ & $2620 \mathrm{a}$ \\
\hline
\end{tabular}




\begin{tabular}{|c|c|c|c|c|c|}
\hline Page & Name & SRM & Page & Name & SRM \\
\hline 60 & $\begin{array}{l}\text { Carbon Dioxide in Nitrogen } \\
\text { (Combustion Efficiency Gas Standard) }\end{array}$ & $2621 \mathrm{a}$ & 106 & $\begin{array}{l}\text { Catalyst Package for Lubricant } \\
\text { Oxidation }\end{array}$ & 1817 \\
\hline 60 & $\begin{array}{l}\text { Carbon Dioxide in Nitrogen } \\
\text { (Combustion Efficiency Gas Standard) }\end{array}$ & $2622 \mathrm{a}$ & 112 & $\begin{array}{l}\text { Centerline Drawings for Optical } \\
\text { Character Recognition, B }\end{array}$ & 1901 \\
\hline 60 & Carbon Dioxide in Nitrogen & $2623 \mathrm{a}$ & & Characters & \\
\hline & (Combustion Efficiency Gas Standard) & & 114 & Centroid Color Chart & 2106 \\
\hline 60 & Carbon Dioxide in Nitrogen & $2624 a$ & 114 & Centroid Color Kit & 2107 \\
\hline 60 & $\begin{array}{l}\text { (Combustion Efficiency Gas Standard) } \\
\text { Carbon Dioxide in Nitrogen }\end{array}$ & $2625 \mathrm{a}$ & 103 & $\begin{array}{l}\text { Cesium-137, Barium-137m Point-Source } \\
\text { Radioactivity Standard }\end{array}$ & $4200 \mathrm{~B}$ \\
\hline & (Combustion Efficiency Gas Standard) & & 103 & Cesium-137, Barium-137m Point-Source & 4207 \\
\hline 60 & $\begin{array}{l}\text { Carbon Dioxide in Nitrogen } \\
\text { (Combustion Efficiency Gas Standard) }\end{array}$ & $2626 a$ & 101 & $\begin{array}{l}\text { Radioactivity Standard } \\
\text { Cesium-137 Burn-Up Standard }\end{array}$ & $4233 \mathrm{~B}$ \\
\hline 60 & $\begin{array}{l}\text { Carbon Dioxide in Nitrogen (Mobile } \\
\text { Source Emission Gas Standard) }\end{array}$ & 2632 & $\begin{array}{l}101 \\
111\end{array}$ & $\begin{array}{l}\text { Cesium-134 Radioactivity Standard } \\
\text { Channel Black Rubber Compound }\end{array}$ & $\begin{array}{l}4250 \mathrm{~B} \\
375 \mathrm{~g}\end{array}$ \\
\hline 60 & $\begin{array}{l}\text { Carbon Dioxide in Nitrogen (Mobile } \\
\text { Source Emission Gas Standard) }\end{array}$ & 2633 & $\begin{array}{l}101 \\
101\end{array}$ & $\begin{array}{l}\text { Chlorine-36 Beta-ray Standard } \\
\text { Chlorine-36 Radioactivity Standard }\end{array}$ & $\begin{array}{l}4943 \\
4422 \mathrm{~L}\end{array}$ \\
\hline 60 & $\begin{array}{l}\text { Carbon Monoxide in Air (Ambient } \\
\text { Air Quality Gas Standard) }\end{array}$ & $2612 \mathrm{a}$ & $\begin{array}{l}56 \\
73\end{array}$ & $\begin{array}{l}\text { Chlorobenzoic Acid } \\
\text { Chrome Refractory }\end{array}$ & $\begin{array}{l}2144 \\
103 a\end{array}$ \\
\hline 60 & $\begin{array}{l}\text { Carbon Monoxide in Air (Ambient } \\
\text { Air Quality Gas Standard) }\end{array}$ & $2613 a$ & 20 & $\begin{array}{l}\text { Chromium-Molybdenum-Aluminum } \\
\text { Steel }\end{array}$ & $106 \mathrm{~B}$ \\
\hline 60 & $\begin{array}{l}\text { Carbon Monoxide in Air (Ambient } \\
\text { Air Quality Gas Standard) }\end{array}$ & $2614 a$ & $\begin{array}{l}20 \\
22\end{array}$ & $\begin{array}{l}\text { Chromium-Molybdenum Steel } \\
\text { Chromium-Molybdenum Steel }\end{array}$ & $\begin{array}{l}36 \mathrm{~b} \\
133 \mathrm{~B}\end{array}$ \\
\hline 59 & Carbon Monoxide in Nitrogen & $1677 \mathrm{c}$ & 20 & Chromium-Nickel-Molybdenum Steel & $139 b$ \\
\hline 59 & Carbon Monoxide in Nitrogen & $1678 \mathrm{c}$ & 26 & Chromium-Nickel-Molybdenum Steel & 1222 \\
\hline 59 & Carbon Monoxide in Nitrogen & $1679 \mathrm{c}$ & 22 & 17Chromium-9 Nickel-0.2 Selenium Steel & 339 \\
\hline 59 & Carbon Monoxide in Nitrogen & $1680 \mathrm{~b}$ & 25 & Chromium-Nickel Spectrographic Steel & $408 \mathrm{a}$ \\
\hline 59 & Carbon Monoxide in Nitrogen & $1681 b$ & & Standard & \\
\hline 60 & $\begin{array}{l}\text { Carbon Monoxide in Nitrogen (Mobile } \\
\text { Source Emission Gas Standard) }\end{array}$ & 2635 & $\begin{array}{l}22 \\
22\end{array}$ & $\begin{array}{l}15 \text { Chromium-7 Nickel Steel } \\
16 \text { Chromium-4 Nickel Steel }\end{array}$ & $\begin{array}{l}344 \\
345\end{array}$ \\
\hline 60 & $\begin{array}{l}\text { Carbon Monoxide in Nitrogen (Mobile } \\
\text { Source Emission Gas Standard) }\end{array}$ & 2636 & 105 & $\begin{array}{l}\text { Chromium-51 Radioactivity Standard } \\
\text { Chromium Steel }\end{array}$ & $\begin{array}{l}4400 L-F \\
163\end{array}$ \\
\hline 60 & $\begin{array}{l}\text { Carbon Monoxide in Nitrogen (Mobile } \\
\text { Source Emission Gas Standard) }\end{array}$ & 2637 & $\begin{array}{l}20 \\
25\end{array}$ & $\begin{array}{l}\text { Chromium-Tungsten Steel } \\
\text { Chromium-Vanadium Spectrographic }\end{array}$ & $\begin{array}{l}155 \\
407 \mathrm{a}\end{array}$ \\
\hline 60 & $\begin{array}{l}\text { Carbon Monoxide in Nitrogen (Mobile } \\
\text { Source Emission Gas Standard) }\end{array}$ & 2638 & 56 & $\begin{array}{l}\text { Steel Standard } \\
\text { Cholesterol }\end{array}$ & $911 \mathrm{a}$ \\
\hline 60 & $\begin{array}{l}\text { Carbon Monoxide in Nitrogen (Mobile } \\
\text { Source Emission Gas Standard) }\end{array}$ & 2639 & $\begin{array}{l}67 \\
57\end{array}$ & $\begin{array}{l}\text { Chrysotile Asbestos Fibers } \\
\text { Citrus Leaves }\end{array}$ & $\begin{array}{l}1876 \\
1572\end{array}$ \\
\hline 60 & $\begin{array}{l}\text { Carbon Monoxide in Nitrogen (Mobile } \\
\text { Source Emission Gas Standard) }\end{array}$ & 2640 & $\begin{array}{l}57 \\
67\end{array}$ & $\begin{array}{l}\text { Clinical Laboratory Thermometer } \\
\text { Cobalt Cyclohexanebutyrate }\end{array}$ & $\begin{array}{l}934 \\
1055 b\end{array}$ \\
\hline 60 & $\begin{array}{l}\text { Carbon Monoxide in Nitrogen (Mobile } \\
\text { Source Emission Gas Standard) }\end{array}$ & 2641 & $\begin{array}{l}23 \\
105\end{array}$ & $\begin{array}{l}\text { Cobalt-Molybdenum-Tungsten Steel } \\
\text { Cobalt-57 Radioactivity Standard }\end{array}$ & $\begin{array}{l}153 \mathrm{~A} \\
4408 \mathrm{~L}-\mathrm{C}\end{array}$ \\
\hline 60 & $\begin{array}{l}\text { Carbon Monoxide in Nitrogen (Mobile } \\
\text { Source Emission Gas Standard) }\end{array}$ & 2642 & $\begin{array}{l}101 \\
46\end{array}$ & $\begin{array}{l}\text { Cobalt- } 60 \text { Radioactivity Standard } \\
\text { Commerical Bronze Standard for }\end{array}$ & $\begin{array}{l}4915 \mathrm{D} \\
1115\end{array}$ \\
\hline 101 & Carbon-14 Radioactivity Standard & 4245 & & Optical Emission and X-ray & \\
\hline 101 & Carbon-14 Radioactivity Standard & 4246 & & Spectroscopic Analysis & \\
\hline 26 & Carbon Steel & 1224 & 46 & Commercial Bronze Standard for & C1115 \\
\hline 19 & Carbon Steel, $0.6 \%$ & $13 \mathrm{~g}$ & & Optical Emission and X-ray & \\
\hline 36 & Cast Iron & $4 \mathrm{k}$ & & Spectroscopic Analysis & \\
\hline 36 & Cast Iron & $5 \mathrm{~L}$ & 46 & Commercial Bronze Standard for & 1116 \\
\hline 36 & Cast Iron & $6 \mathrm{~g}$ & & Optical Emission and X-ray & \\
\hline 36 & Cast Iron & $7 \mathrm{G}$ & & Spectroscopic Analysis & \\
\hline 36 & Cast Iron Car Wheel & $122 \mathrm{~h}$ & & & \\
\hline 38 & Cast Steel 3 & C1173 & & & \\
\hline 38 & Cast Steel Standard & $1138 \mathrm{a}$ & & & \\
\hline 38 & Cast Steel Standard & $1139 \mathrm{a}$ & & & \\
\hline
\end{tabular}




\begin{tabular}{|c|c|c|c|c|c|}
\hline Page & Name & SRM & Page & Name & SRM \\
\hline \multirow[t]{2}{*}{46} & $\begin{array}{l}\text { Commercial Bronze Standard for } \\
\text { Optical Emission and X-ray }\end{array}$ & $\mathrm{C} 1116$ & 39 & $\begin{array}{l}\text { Cupro-Nickel, } 10 \% \text { (CDA 706) High } \\
\text { Purity }\end{array}$ & 874 \\
\hline & Spectroscopic Analysis & & 55 & Cystine & $143 \mathrm{c}$ \\
\hline \multirow[t]{3}{*}{46} & Commercial Bronze Standard for & 1117 & 55,100 & Dextrose & $41 b$ \\
\hline & Optical Emission and X-ray & & 56 & D-Glucose & 917 \\
\hline & Spectroscopic Analysis & & 67 & Dibutyltin Bis(2-ethylhexanoate) & $1057 b$ \\
\hline 46 & $\begin{array}{l}\text { Commercial Bronze Standard for } \\
\text { Optical Emission and X-ray } \\
\text { Spectroscopic Analysis }\end{array}$ & $\mathrm{C} 1117$ & 96 & $\begin{array}{l}\text { Didymium Glass Filter for Checking } \\
\text { the Wavelength Scale of } \\
\text { Spectrophotometers }\end{array}$ & 2009 \\
\hline 80 & Common Lead Isotopic Standard & 981 & 96 & Didymium Glass Fitler for Checking & 2010 \\
\hline 69 & Copper Concentrate & 332 & & the Wavelength Scale of & \\
\hline 116 & Copper Heat Capacity Test Specimen & RM5 & & Spectrophotometers & \\
\hline 36 & Copper-Nickel-Chromiun Cast Iron & $115 \mathrm{~A}$ & 83 & Disodium Hydrogen Phosphate & $1861 \mathrm{Ic}$ \\
\hline 69 & Copper Ore, Mill Heads & 330 & 84 & Disodium Hydrogen Phosphate & $2186 \mathrm{II}$ \\
\hline 69 & Copper Ore, Mill Tails & 331 & 56 & D-Mannitol & 920 \\
\hline 95 & Copper-Thermal Expansion & $736 a$ & 72 & Dolomitic Limestone & $88 \mathrm{a}$ \\
\hline \multirow[t]{2}{*}{92} & Copper, Secondary Freezing Point & $45 d$ & 52 & Doped Platinum & $681 \mathrm{Ll}$ \\
\hline & Standard & & 52 & Doped Platinum & $681 \mathrm{~L} 2$ \\
\hline 56 & Cortisol (Hydrocortisone) & 921 & 36 & Ductile Cast Iron & 341 \\
\hline 56 & Creatinine & 914 & 52,109 & Electrical Residual Resistivity Ratio & 769 \\
\hline 26 & Cr-Mo Low Alloy Steel & 1270 & & Standard & \\
\hline 20 & Cr-Mo Steel (ASTM A-213) & 291 & 36 & Electrolytic Iron & 365 \\
\hline \multirow[t]{2}{*}{25} & Cr-Mo (SAE 4140) Spectrographic & 414 & 26 & Electrolytic Iron & $1265 \mathrm{a}$ \\
\hline & Steel Standard & & 94,108 & Electrolytic Iron, Thermal & 1463 \\
\hline 25 & $\begin{array}{l}\text { Cr-Mo (SAE 4150) Spectrographic } \\
\text { Steel Standard }\end{array}$ & 427 & & $\begin{array}{l}\text { Conductivity and Electrical } \\
\text { Resistivity }\end{array}$ & \\
\hline 25 & $\begin{array}{l}\text { Cr-Mo (SAE X4130) Spectrographic } \\
\text { Steel Standard }\end{array}$ & $418 \mathrm{a}$ & 94,108 & $\begin{array}{l}\text { Electrolytic Iron, Thermal } \\
\text { Conductivity and Electrical }\end{array}$ & 1464 \\
\hline 20 & Cr-Ni-Mo Steel (AISI 8620) & 293 & & Resistivity & \\
\hline 23 & 18Cr-10Ni Steel (AISI 304L) & $101 \mathrm{f}$ & 49 & Electronic and Magnetic Alloy & 1159 \\
\hline 20 & Cr-V Steel (Modified) & 363 & & Standard & \\
\hline 26 & Cr-V Steel (Modified) & $1263 a$ & 49 & Electronic and Magnetic Alloy & 1160 \\
\hline 20 & Cr.V Steel (SAE 6150) & $30 f$ & & Standard & \\
\hline 57,96 & Crystalline Potassium Dichromate & 935 & 80 & Enriched Boric Acid & 952 \\
\hline \multirow[t]{3}{*}{96} & Crystalline Potassium Iodide, & 2032 & 80 & Equal-Atom Lead Isotopic Standard & 982 \\
\hline & Heterochromatic Stray Radiant & & 62 & Estuarine Sediment & 1646 \\
\hline & Energy Standard & & 103 & Europium-152 Point-Source Standard & $4218 \mathrm{E}$ \\
\hline \multirow[t]{3}{*}{96} & Crystalline (Ruby) Electron & 2601 & 101 & Europium-152 Radioactivity Standard & $4370 \mathrm{~B}$ \\
\hline & Paramagnetic Resonance & & 88,89 & Extra Dense Lead Glass & 709 \\
\hline & Absorption Intensity Standard & & 53 & Fe-Cr-Ni Alloy Microprobe Standard & $479 a$ \\
\hline 46 & Cupro-Nickel (CDA 706) & 1275 & 54 & Fe-3Si Alloy Microprobe Standard & 483 \\
\hline 46 & Cupro-Nickel (CDA 715) & 1276 & 72 & Feldspar & $70 \mathrm{a}$ \\
\hline \multirow[t]{10}{*}{39} & Cupro-Nickel, 10\% (CDA 706) Doped & 875 & 72 & Feldspar & $99 a$ \\
\hline & & & 35 & Ferrochromium (Low Carbon) & 196 \\
\hline & & & 35 & Ferrochromium Silicon & 689 \\
\hline & & & 35 & Ferroniobium & 340 \\
\hline & & & 35 & Ferrophosphorus & 90 \\
\hline & & & 35 & Ferrosilicon & $58 \mathrm{a}$ \\
\hline & & & 35 & Ferrosilicon & $59 a$ \\
\hline & & & 35 & Ferrosilicon $(75 \% \mathrm{Si})$ & 195 \\
\hline & & & 98 & $\begin{array}{l}\text { First Surface Aluminum Mirror for } \\
\text { Specular Reflectance }\end{array}$ & $2003 a$ \\
\hline & & & 98 & First Surface Mirror, Gold on Glass & $2008 \mathrm{a}$ \\
\hline
\end{tabular}




\begin{tabular}{|c|c|c|c|c|c|}
\hline Page & Name & SRM & Page & Name & SRM \\
\hline 80 & Fission Track Glass Standard & 961 & 105 & Gold-198 Radioactivity Standard & $4405 \mathrm{~L} \cdot \mathrm{B}$ \\
\hline 80 & Fission Track Glass Standard & $962 \mathrm{a}$ & 54 & Gold-Silver Wires for Microprobe & 481 \\
\hline 80 & Fission Track Glass Standard & $963 \mathrm{a}$ & & Analysis & \\
\hline 80 & Fission Track Glass Standard & 964 & 94 & Gold, Vapor Pressure & 745 \\
\hline 72 & Flint Clay & $97 \mathrm{a}$ & 36 & Gray Cast Iron & 334 \\
\hline 56 & Fluorobenzoic Acid & 2143 & 64 & Halocarbons (in methanol) for Water & 1639 \\
\hline 69 & Fluorspar & $79 \mathrm{a}$ & & Analysis & \\
\hline 46 & Free-Cutting Brass & 1103 & 31 & High-Alloy Steel (A-743) & C1288 \\
\hline 46 & Free-Cutting Brass & C1104 & 31 & High-Alloy Steel (AISI 310 Mod.) & C1287 \\
\hline 66 & Freeze-Dried Urine & 2670 & 31 & High-Alloy Steel, (AISI 414 Mod.) & C1289 \\
\hline \multirow[t]{2}{*}{66} & Freeze-Dried Urine Certified & $2671 \mathrm{a}$ & 36 & High-Alloy White Cast & 892 \\
\hline & for Fluoride & & 36 & High-Alloy White Cast Iron & 890 \\
\hline \multirow[t]{2}{*}{66} & Freeze-Dried Urine Certified & $2672 a$ & 36 & High-Alloy White Cast Iron & 891 \\
\hline & for Mercury & & 35 & High-Carbon Ferrochromium & $64 c$ \\
\hline 95 & Fused-Silica Thermal Expansion & 739 & 35 & High-Carbon Ferromanganese & $68 c$ \\
\hline \multirow[t]{2}{*}{102} & Gadolinium-148 Alpha-Particle & 4907 & 20 & High-Carbon Steel (Modified) & 364 \\
\hline & Standard & & 26 & High-Carbon Steel (Modified) & $1264 \mathrm{a}$ \\
\hline 57,93 & Gallium Melting-Point Standard & 1968 & 69 & High-Grade Fluorspar & 180 \\
\hline 105 & Gallium-67 Radioactivity Standard & 4416L-D & 22 & High-Nickel Steel & $126 \mathrm{c}$ \\
\hline 111 & Gas Furnace Black Rubber Compound & $382 \mathrm{a}$ & 32 & High-Nickel Steel & 1158 \\
\hline 51 & Gasometric Set (1095-1099) & 1089 & 52 & High-Purity Gold & 685 \\
\hline \multirow[t]{2}{*}{51} & Gasometric Standard for Unalloyed & 357 & 52 & High-Purity Platinum & $680 \mathrm{~L} 1 \mathrm{~A}$ \\
\hline & Zirconium & & 52 & High-Purity Platinum & $680 \mathrm{~L} 2 \mathrm{~A}$ \\
\hline \multirow[t]{2}{*}{51} & Gasometric Standard for Unalloyed & 358 & 95 & High-Purity Platinum Thermoelement & 1967 \\
\hline & Zirconium & & 52 & High-Purity Zinc & 682 \\
\hline \multirow[t]{2}{*}{64} & Generator Columns for Polynuclear & 1644 & 20 & High-Silicon Steel & 179 \\
\hline & Aromatic Hydrocarbons & & 26 & High-Silicon Steel & 1134 \\
\hline 46 & Gilding Metal & 1112 & 26 & High-Silicon Steel & 1135 \\
\hline 46 & Gilding Metal & C1112 & 20 & High-Silicon Steel (Calcium Bearing) & $125 b$ \\
\hline 46 & Gilding Metal & 1113 & 20 & High-Sulfur Steel & 105 \\
\hline 46 & Gilding Metal & C1113 & 20 & High-Sulfur Steel & $129 \mathrm{c}$ \\
\hline 46 & Gilding Metal & 1114 & 26 & High-Sulfur Steel & 1136 \\
\hline 46 & Gilding Metal & C1114 & 22 & High Temperature Alloy A286 & 348 \\
\hline 53 & Glasses for Microchemical Analysis & 1871 & 33 & High Temperature Alloy M308 & 1197 \\
\hline 53 & Glasses for Microchemical Analysis & 1872 & 33 & High Temperature Alloy L605 and & S1199 \\
\hline 53 & Glasses for Microchemical Analysis & 1873 & & S 816 & \\
\hline 53 & Glasses for Microchemical Analysis & 1874 & 33 & High-Temperature Alloy & $1206-2$ \\
\hline 53 & Glasses for Microchemical Analysis & 1875 & 33 & High-Temperature Alloy & 1207.1 \\
\hline 117 & Glass Fibers for Microanalysis & RM 31 & 33 & High-Temperature Alloy & $1207-2$ \\
\hline \multirow[t]{2}{*}{96} & Glass Filter for Transmittance & 2030 & 33 & High-Temperature Alloy & $1208-1$ \\
\hline & Measurement & & 33 & High-Temperature Alloy & $1208-2$ \\
\hline 56,96 & Glass Filters for Spectrophotometry & $930 \mathrm{D}$ & 105,117 & Homogeneous River Sediment for & RM 45B \\
\hline 107 & Glass Fluorescence Source & 477 & & Radioactivity Measurements & \\
\hline 72 & Glass Sand & $81 \mathrm{a}$ & 195 & Human Liver, Environmental & 4352 \\
\hline 72 & Glass Sand & $165 a$ & & Radioactivity & \\
\hline 113 & Glass Spheres & $1019 \mathrm{a}$ & 105 & Human Lung, Environmental & 4351 \\
\hline 87 & Gold Coating on Glass Sealing Alloy & $1398 \mathrm{a}$ & & Radioactivity & \\
\hline 87 & Gold Coating on Nickel & 1379 & 56 & Human Serum & 909 \\
\hline 87 & Gold Coating on Nickel & 1380 & & & \\
\hline 87 & Gold Coating on Nickel & $1399 b$ & & & \\
\hline 54 & $\begin{array}{l}\text { Gold-Copper Wires for Microprobe } \\
\text { Analysis }\end{array}$ & 482 & & & \\
\hline 105 & Gold-195 Radioactivity Standard & $4421 \mathrm{~L}$ & & & \\
\hline
\end{tabular}




\begin{tabular}{|c|c|c|c|c|c|}
\hline Page & Name & SRM & Page & Name & SRM \\
\hline 51 & Hydrogen in Unalloyed Titanium & $352 b$ & 70 & Iron Ore (Sibley) & $27 f$ \\
\hline 51 & Hydrogen in Unalloyed Titanium & 1086 & 70 & Iron Ore Concentrate (Canada) & 690 \\
\hline 51 & Hydrogen in Unalloyed Titanium & 1087 & 105 & Iron-59 Radioactivity Standard & $4411 \mathrm{~L} \cdot \mathrm{B}$ \\
\hline 51 & Hydrogen in Unalloyed Titanium & 1088 & 111 & Isobutylene-Isoprene (Butyl) Rubber & 1495 \\
\hline 101,102 & Hydrogen-3 Radioactivity Standard & 4361 & 111 & Isobutylene-Isoprene (Butyl) Rubber & $388 \mathrm{~L}$ \\
\hline 101 & Hydrogen-3 Radioactivity Standard & $4926 \mathrm{C}$ & 80 & Isotopic Standard for Bromine & 977 \\
\hline \multirow[t]{2}{*}{101} & Hydrogen- 3 Toluene Radioactivity & 4947 & 80 & Isotopic Standard for Chlorine & 975 \\
\hline & Standard & & 80 & Isotopic Standard for Chromium & 979 \\
\hline \multirow[t]{2}{*}{56} & 4-Hydroxy-3 methoxy-DL-mandelic & 925 & 80 & Isotopic Standard for Copper & 976 \\
\hline & Acid (VMA) & & 80 & Isotopic Standard for Magnesium & 980 \\
\hline \multirow[t]{2}{*}{118} & ICTA High Temperature Set & GM 760 & 80 & Isotopic Standard for Silver & 978 \\
\hline & Differential Thermal Analysis & & 103 & Krypton-85 Gaseous Radioactivity & $4308 \mathrm{C}$ \\
\hline \multirow[t]{2}{*}{118} & ICTA Low Temperature Set Differen- & GM 757 & & Standard & \\
\hline & tial Thermal Analysis & & 103 & Krypton-85 Radioactivity Standard & 4235 \\
\hline \multirow[t]{2}{*}{118} & ICTA Mod Temperature Set Differen- & GM 759 & 103 & Krypton-85 Radioactivity Standard & $4935 \mathrm{C}$ \\
\hline & tial Thermal Analysis & & 74 & Lead-Barium Glass & 89 \\
\hline \multirow[t]{2}{*}{118} & ICTA Mid Temperature Set Differen- & GM 758 & 42 & Lead-Base Bearing Metal & $53 \mathrm{e}$ \\
\hline & tial Thermal Analysis & & 49 & Lead-Base Bearing Metal & 1132 \\
\hline \multirow[t]{2}{*}{118} & ICTA Polystyrene Differential & GM 754 & 67 & Lead Cyclohexanebutyrate & $1059 \mathrm{c}$ \\
\hline & Thermal Analysis & & 62 & Lead in Reference Fuel & $1636 a$ \\
\hline 118 & ICTA Thermogravimetry Set & GM 761 & 62 & Lead in Reference Fuel & $1637 \mathrm{a}$ \\
\hline 33 & Incoloy, 901 and Hastelloy $\mathrm{X}$ & S1 198 & 62 & Lead in Reference Fuel & $1638 \mathrm{a}$ \\
\hline 42 & Inconels, Alloy 600 (Chips) & 864 & 56 & Lead Nitrate & 928 \\
\hline 33 & Inconels, Alloy 600 (Solid) & 1244 & 66 & Lead on Filter Media & 2674 \\
\hline 42 & Inconels, Alloy 625 (Chips) & 865 & 105 & Lead-203 Radioactivity Standard & $4420 \mathrm{~L}$ \\
\hline 33 & Inconels, Alloy 625 (Solid) & 1245 & 92 & Lead, Secondary Freezing Point & $49 \mathrm{e}$ \\
\hline 42 & Incoloy, Alloy 800 (Chips) & 866 & & Standard & \\
\hline 33 & Incoloy, Alloy 800 (Solid) & 1246 & 90 & Lead-Silica Glass & 1827 \\
\hline 42 & Incoloy, Alloy 825 (Chips) & 867 & 88 & Lead-Silica Glass (Viscosity) & 711 \\
\hline 33 & Incoloy, Alloy 825 (Solid) & 1247 & 88,109 & Lead-Silica Glass for dc Volume & 624 \\
\hline 105 & Indium-111 Radioactivity Standard & 4417 L-C & & Resistivity & \\
\hline 25 & $\begin{array}{l}\text { Ingot Iron Spectrographic Steel } \\
\text { Standard }\end{array}$ & $420 \mathrm{a}$ & 88 & $\begin{array}{l}\text { Lead-Silica Glass for Dielectric } \\
\text { Constant }\end{array}$ & 774 \\
\hline 44 & Intermediate Purity Selenium & 726 & 80 & Lead 206 Spike Assay and Isotopic & 991 \\
\hline 45 & Intermediate-Purity Zinc & 728 & & Solution Standard & \\
\hline 105 & Iodine-123 Radioactivity Standard & $4414 \mathrm{~L}-\mathrm{C}$ & 39 & Leaded-Tin Bronze Alloy & 1035 \\
\hline 105 & Iodine-125 Radiactivity Standard & $4407 \mathrm{~L}-\mathrm{H}$ & 114 & Light-Sensitive Paper & $700 \mathrm{~d}$ \\
\hline 101 & Iodine-129 Radioactivity Standard & $4949 B$ & 114 & Light-Sensitive Paper & $701 d$ \\
\hline 105 & Iodine-131 Radioactivity Standard & 4401L-I & 114 & Light-Sensitive Plastic Chip & 703 \\
\hline 106 & Iron Foil Mössbauer Standard & 1541 & 90,94 & Linear Polyethylene & 1475 \\
\hline 104 & Iron-55 Low-Energy Photon Standard & $4260 \mathrm{C}$ & 90 & Linear Polyethylene & 1482 \\
\hline 57 & Iron Metal (Clinical Standard) & 937 & 90 & Linear Polyethylene & 1483 \\
\hline 70 & Iron Ore (Labrador) & 692 & 90 & Linear Polyethylene & 1484 \\
\hline \multirow[t]{12}{*}{70} & Iron Ore (Nimba) & 693 & 116 & $\begin{array}{l}\text { Linerboard, Standard for Tape } \\
\text { Adhesion Testing }\end{array}$ & 1810 \\
\hline & & & 56,96 & $\begin{array}{l}\text { Liquid Absorbance Standard for } \\
\text { Ultraviolet and Visible } \\
\text { Spectrophotometry }\end{array}$ & $931 \mathrm{c}$ \\
\hline & & & 56 & Lithium Carbonate & 924 \\
\hline & & & 69 & Lithium Ore & 181 \\
\hline & & & 69 & Lithium Ore & 182 \\
\hline & & & 69 & Lithium Ore & 183 \\
\hline & & & 26 & Low-Alloy Steel, (AISI 4130) & 1225 \\
\hline & & & 26 & Low Alloy Steel & 1226 \\
\hline & & & 26 & Low Alloy Steel (A242 Mod.) & C1285 \\
\hline & & & 20 & Low-Alloy Steel, AISI 4130 & $72 \mathrm{~g}$ \\
\hline & & & 26 & Low Alloy Steel (AISI 1526, Modified) & 1269 \\
\hline & & & 26 & Low-Alloy Steel (Hy 80) & 1286 \\
\hline
\end{tabular}


Low-Alloy Steel Set (661-665)

Low-Carbon Silicon Steel

Low-Carbon Stainless Steel (AISI 316L)

Magnesium-base Alloy

Magnesium Cyclohexanebutyrate

Magnesium Gluconate Dihydrate

Magnetic Coating on Magnetic

Substrate (Nickel on Steel)

Magnetic Coating on Magnetic

Substrate (Nickel on Steel)

Magnetic Coating on Non-Magnetic Substrate (Nickel and Chromium on brass

112 Magnetic Tape, High Density

Manganese Fluoride, Magnetic Gram Susceptibility

$1366 \mathrm{a} \quad 20$

Manganese-54 Point-Source

Radioactivity Standard

Manganese-54 Radioactivity Standard

Manganese Steel

Manganous Cyclohexanebutyrate

Maraging Steel

Metal on Quartz Filters for Spectrophotometry

Metals on Filter Media

Methane in Air

Methane in Air

Methane in Air

Medium Manganese Spectrographic Steel Standard

Mercaptobenzothiazole

Mercury, Freezing Point

Mercury-203 Radioactivity Standard

Mercury in Water, $\mu \mathrm{g} / \mathrm{mL}$

Mercury in Water, $\mathrm{ng} / \mathrm{mL}$

Microcopy Resolution Test Chart

Microprobe Standard - Cartridge Brass

Mineral Glasses for Microanalysis

Molybdenum Concentrate

Molybdenum, Heat Capacity

Molybdenum-99 Radioactivity

Standard

Molybdenum-Tungsten-Chromium-

Vanadium Steel

Naval Brass Standards for Optical

Emission and Spectroscopic

Analysis

Naval Brass Standards for Optical

Emission and Spectroscopic

Analysis

Naval Brass Standards for Optical

Emission and Spectroscopic

Analysis

Naval Brass Standards for Optical

Emission and Spectroscopic

Analysis
S668

$131 \mathrm{c}$

1036

$166 \mathrm{c}$

46

171

1061c 88

$929 \quad 79$

$1365 \mathrm{a} \quad 36$

$1367 a-67$

$766 \quad 101$

$25 \mathrm{~d}$

Naval Brass Standards for Optical

1108

Emission and Spectroscopic

Analysis

Naval Brass Standards for Optical

Emission and Spectroscopic

Analysis

Neutral Glass

Neutron Density Monitor Wire

953

Nickel-Chromium Cast Iron $82 \mathrm{~b}$

Nickel-Chromium-Molybdenum Cast 1ron $107 \mathrm{C}$

Nickel-Chromium Steel 32E

Nickel-Copper Alloy

Nickel Cyclohexanebutyrate

Nickel Oxide, No. 1

882

Nickel Oxide, No. 2

$1065 b$

Nickel Oxide, No. 3

671

672

673

Nickel-63 Radioactivity Standard 4226

Nickel Silver (CDA 762)

Nickel Siver (CDA 770)

Nickel Spectrographic Steel Standard

879

880

$409 b$

772

Nickel Sphere, Magnetic Moment

Nickel Steel

$\mathrm{Ni}$-Cr-Mo-V Steel

Nicotinic Acid

Niobium-94 Gamma-ray Standard

Nitric Oxide in Nitrogen

Nitric Oxide in Nitrogen

Nitric Oxide in Nitrogen

Nitric Oxide in Nitrogen

Nitric Oxide in Nitrogen

Nitric Oxide in Nitrogen (Mobile

Source Emission Gas Standard)

Nitric Oxide in Nitrogen (Mobile

Source Emission Gas Standard)

Nitric Oxide in Nitrogen (Mobile

Source Emission Gas Standard)

$4418 \mathrm{~L}$

$1641 \mathrm{~b} \quad 60$

$1642 b$

$1010 \mathrm{a} \quad 60$

478

470

333

781

4412L-H

$134 \mathrm{~A}$

Nitric Oxide in Nitrogen (Mobile Source Emission Gas Standard)

Nitric Oxide in Nitrogen (Mobile Source Emission Gas Standard)

Nitrogen Dioxide in Air (Stationary

Source Emission Gas Standard)

Nitrogen Dioxide in Air (Stationary Source Emission Gas Standard)

Nitrogen Dioxide in Air (Stationary Source Emission Gas Standard)

Nitrogen Dioxide in Air (Stationary

$33 \mathrm{~d}$

1173

148

$4201 \mathrm{~B}$

$1683 b$

$1684 \mathrm{~b}$

$1685 \mathrm{~b}$

$1686 \mathrm{~b}$

$1687 \mathrm{~b}$

2627

2628

2629

2630

2631

2653

2654

2655

2656

Source Emission Gas Standard)

61

Nitrogen Dioxide Permeation Device

$1629 \mathrm{a}$

4-Nitrophenol 


\begin{tabular}{|c|c|}
\hline Page & Name \\
\hline 36 & Nodular Cast 1ron \\
\hline 59,113 & $\begin{array}{l}\text { Nominal One Micrometer Polystyrene } \\
\text { Spheres }\end{array}$ \\
\hline 57 & Non-Fat Powdered Milk \\
\hline 86 & $\begin{array}{l}\text { Nonmagnetic Coating or Magnetic } \\
\text { Substrate (Copper and Chromium } \\
\text { on Steel) }\end{array}$ \\
\hline 86 & $\begin{array}{l}\text { Nonmagnetic Coating on Magnetic } \\
\text { Substrate (Copper and Chromium } \\
\text { on Steel) }\end{array}$ \\
\hline 85 & $\begin{array}{l}\text { Nonmagnetic Coating on Magnetic } \\
\text { Substrate (Copper and Chromium } \\
\text { on Steel) }\end{array}$ \\
\hline 85 & $\begin{array}{l}\text { Nonmagnetic Coating on Magnetic } \\
\text { Substrate (Copper and Chromium } \\
\text { on Steel) }\end{array}$ \\
\hline 86 & $\begin{array}{l}\text { Nonmagnetic Coating on Magnetic } \\
\text { Substrate (Copper and Chromium } \\
\text { on Steel) }\end{array}$ \\
\hline 86 & $\begin{array}{l}\text { Nonmagnetic Coating on Magnetic } \\
\text { Substrate (Copper and Chromium } \\
\text { on Steel) }\end{array}$ \\
\hline 119 & NPL GM Alpha Alumina \\
\hline 119 & NPL GM Alpha Alumina \\
\hline 119 & NPL GM Alpha Alumina \\
\hline 119 & NPL GM Alpha Alumina \\
\hline 119 & NPL GM Graphitized Carbon Black \\
\hline 119 & NPL GM Graphitized Carbon Black \\
\hline 119 & NPL GM Melting Point Set \\
\hline 119 & NPL GM Non-porous Silica \\
\hline 119 & NPL GM Non-porous Silica \\
\hline 111 & $\begin{array}{l}\text { N-tertiary-Butyl-2-benzothiazolesulfen- } \\
\text { amide Rubber Compound }\end{array}$ \\
\hline 72 & Obsidian Rock \\
\hline 67 & Octaphenylcyclotetrasiloxane \\
\hline 111 & Oil Furnace Black Rubber Compound \\
\hline 74 & Opal Glass Powder \\
\hline & Optical Emission and X-ray \\
\hline 46 & Spectroscopic Analysis \\
\hline & Optical Microscope Linewidth \\
\hline 85 & Measurement Standard \\
\hline & Optical Microscope Linewidth \\
\hline 85 & Measurement Standard \\
\hline 85 & $\begin{array}{l}\text { Optical Microscope Linewidth } \\
\text { Measurement Standard }\end{array}$ \\
\hline
\end{tabular}

\begin{tabular}{|c|c|c|c|}
\hline SRM & Page & Name & SRM \\
\hline $342 \mathrm{a}$ & 64 & Organics in Shale Oil & 1580 \\
\hline \multirow[t]{2}{*}{1690} & 102 & Oxalic Acid & $4990 \mathrm{C}$ \\
\hline & 51 & $\begin{array}{l}\text { Oxygen in Ferrous Materials } \\
\text { Ingot Iron }\end{array}$ & 1090 \\
\hline 1359 & 51 & $\begin{array}{l}\text { Oxygen in Ferrous Materials } \\
\text { (Stainless Steel AISI 431) }\end{array}$ & 1091 \\
\hline \multirow[t]{3}{*}{1360} & 51 & $\begin{array}{l}\text { Oxygen in Ferrous Materials Vacuum } \\
\text { Melted Steel }\end{array}$ & 1092 \\
\hline & 51 & Oxygen in Maraging Steel & 1094 \\
\hline & 60 & Oxygen in Nitrogen (Gas Standard) & 2657 \\
\hline \multirow{3}{*}{$1361 \mathrm{~b}$} & 60 & Oxygen in Nitrogen (Gas Standard) & 2658 \\
\hline & 60 & Oxygen in Nitrogen (Gas Standard) & 2659 \\
\hline & 51 & Oxygen in Titanium-Base Materials & 355 \\
\hline \multirow[t]{3}{*}{$1362 \mathrm{a}$} & 51 & Oxygen in Valve Steel & 1093 \\
\hline & 57 & Oyster Tissue & 1566 \\
\hline & 95 & $\begin{array}{l}\text { Palladium, Magnetic Gram } \\
\text { Susceptibility }\end{array}$ & 765 \\
\hline $1363 \mathrm{a}$ & 109 & Penetrant Test Block & 1850 \\
\hline \multirow{3}{*}{$1364 \mathrm{a}$} & 105 & $\begin{array}{l}\text { Peruvian Soil, Environmental } \\
\text { Radioactivity }\end{array}$ & 4355 \\
\hline & 64 & Petroleum Crude Oil & 1582 \\
\hline & 68,71 & Phosphate Rock (Florida) & $120 \mathrm{~b}$ \\
\hline 8005 & 39 & Phosphor Bronze (CDA 521) & 871 \\
\hline 8006 & 39 & Phosphor Bronze (CDA 544) & 872 \\
\hline 8007 & 31 & Phosphorized Copper, Cu VIII & $\mathrm{C} 1251$ \\
\hline 8008 & 31 & Phosphorized Copper, Cu IX & $\mathrm{C} 1252$ \\
\hline 8001 & 31 & Phosphorized Copper, $\mathrm{Cu}$ X & $\mathrm{C} 1253$ \\
\hline 8002 & 105 & Phosphorus-32 Radioactivity Standard & 4406L-G \\
\hline 8000 & 115 & Photographic Step Tablet & 1008 \\
\hline 8003 & 57 & Pine Needles & 1575 \\
\hline 8004 & 72 & Plastic Clay & $98 \mathrm{a}$ \\
\hline $384 d$ & 95 & $\begin{array}{l}\text { Platinum, Magnetic Gram } \\
\text { Susceptibility }\end{array}$ & 764 \\
\hline \multirow{4}{*}{$\begin{array}{l}278 \\
1066 a \\
378 b \\
91 \\
1102\end{array}$} & 102 & Plutonium-238 Alpha-Particle Standard & $4906 \mathrm{~B}$ \\
\hline & 101 & $\begin{array}{l}\text { Plutonium-240 Alpha-Particle Emission- } \\
\text { Rate Solution Standard }\end{array}$ & 4338 \\
\hline & 101 & $\begin{array}{l}\text { Plutonium-239 Alpha-Particle Solution } \\
\text { Standard }\end{array}$ & 4331 \\
\hline & 101 & $\begin{array}{l}\text { Plutonium-242 Alpha-Particle Solution } \\
\text { Standard }\end{array}$ & 4334B \\
\hline 474 & 77 & Plutonium Isotopic Standard & 946 \\
\hline \multirow[t]{2}{*}{475} & 77 & Plutonium Isotopic Standard & 947 \\
\hline & 77 & Plutonium Isotopic Standard & 948 \\
\hline \multirow[t]{13}{*}{476} & 55,77 & Plutonium Metal & $949 f$ \\
\hline & 77 & $\begin{array}{l}\text { Plutonium Metal (Standard Matrix } \\
\text { Material) }\end{array}$ & 945 \\
\hline & 77 & $\begin{array}{l}\text { Plutonium-244 Spike Assay and } \\
\text { Isotopic Standard }\end{array}$ & 996 \\
\hline & 65 & Polychlorinated Biphenyls in Oil & 1581 \\
\hline & 89 & $\begin{array}{l}\text { Polycrystalline Alumina Elasticity } \\
\text { Standard }\end{array}$ & 718 \\
\hline & 108 & $\begin{array}{l}\text { Polyester Plastic Film for Oxygen } \\
\text { Gas Transmission }\end{array}$ & 1470 \\
\hline & 91 & Polyisobutylene Solution in Cetane & 1490 \\
\hline & 90 & Polystyrene & 1478 \\
\hline & 90 & Polystyrene & 1479 \\
\hline & 90 & Polystyrene (Broad Molecular Weight) & 706 \\
\hline & 90,94 & $\begin{array}{l}\text { Polystyrene (Narrow Molecular } \\
\text { Weight) }\end{array}$ & 705 \\
\hline & 59,113 & Polystyrene Spheres & 1691 \\
\hline & 75 & Portland Cement (Black) & 1880 \\
\hline
\end{tabular}




\begin{tabular}{|c|c|c|c|c|c|}
\hline Page & Name & SRM & Page & Name & SRM \\
\hline 75 & Portland Cement (Blue) & 635 & 66 & Quartz on Filter Media & $2679 a$ \\
\hline 75 & Portland Cement (Clear) & 639 & 57,96 & Quinine Sulfate Dihydrate & 936 \\
\hline 75 & Portland Cement (Gold) & 634 & 80 & Radiogenic Lead Isotopic Standard & 983 \\
\hline 75 & Portland Cement (Green) & 638 & 104 & Radium-226 Gamma-ray Standard & 4956 \\
\hline 75 & Portland Cement (Pink) & 637 & 104 & Radium-226 Gamma-ray Standard & 4957 \\
\hline 75 & Portland Cement (Red) & 633 & 104 & Radium-226 Gamma-ray Standard & 4958 \\
\hline 75 & Portland Cement (White) & 1881 & 104 & Radium-226 Gamma-ray Standard & 4959 \\
\hline 75 & Portland Cement (Yellow) & 636 & 104 & Radium-226 Gamma-ray Standard & 4960 \\
\hline 113 & Portland Cement Fineness Standard & $114 n$ & 104 & Radium-226 Gamma-ray Standard & 4961 \\
\hline 84 & Potassium Chloride & 2202 & 104 & Radium-226 Gamma-ray Standard & 4962 \\
\hline 56 & Potassium Chloride (Clinical Standard) & 918 & 104 & Radium-226 Gamma-ray Standard & 4963 \\
\hline \multirow[t]{2}{*}{55} & Potassium Chloride (Primary & 999 & 104 & Radium-226 Gamma-ray Standard & 4964B \\
\hline & Chemical) & & 104 & Radium Standard (Blank Solution) & $4952 B$ \\
\hline \multirow[t]{2}{*}{93} & Potassium Chloride for Solution & 1655 & 104 & Radon-226 for Radon Analysis & $4953 \mathrm{C}$ \\
\hline & Calorimetry & & 46 & Red Brass & 1109 \\
\hline 55 & Potassium Dichromate & $136 \mathrm{~d}$ & 46 & Red Brass & $\mathrm{Cl} 109$ \\
\hline 68 & Potassium Dihydrogen Phosphate & 200 & 46 & Red Brass & 1110 \\
\hline 83 & Potassium Dihydrogen Phosphate & $186 \mathrm{Ic}$ & 46 & Red Brass & $\mathrm{C} 1110$ \\
\hline 84 & Potassium Dihydrogen Phosphate & $2186 \mathrm{I}$ & 46 & Red Brass & 1111 \\
\hline 67 & Potassium Erucate & 1076 & 46 & Red Brass & $\mathrm{C} 1111$ \\
\hline 76 & Potassium Feldspar & 607 & 70 & Reduced Iron Oxide & 691 \\
\hline 84 & Potassium Fluoride & 2203 & 108 & Reference Fuel Isooctane & $1816 a$ \\
\hline 83 & Potassium Hydrogen Phthalate & $185 \mathrm{e}$ & 108 & Reference Fuel n-Heptane & $1815 a$ \\
\hline 83 & Potassium Hydrogen Tartrate & 188 & 115 & Reflection Step Tablet & 2061 \\
\hline 96 & Potassium Iodide with Attenuator & 2033 & 99 & Refractive Index Glass & 1820 \\
\hline 68 & Potassium Nitrate & 193 & 99 & Refractive Index Silicone Liquids & 1823 \\
\hline 83 & Potassium Tetroxalate & 189 & 99 & Refractive Index, Soda-Lime Glass & 1822 \\
\hline 61 & Powdered Lead Based Paint & 1579 & 89 & Relative Stress-Optical Coefficient & 708 \\
\hline \multirow[t]{3}{*}{64} & Priority Pollutant Polynuclear & 1647 & & Glass & \\
\hline & Aromatic Hydrocarbons (in & & 26 & Resulfurized-Rephosphorized Steel & $\mathrm{C} 1221$ \\
\hline & Acetonitrile) & & 57 & Rice Flour & 1568 \\
\hline 59 & Propane in Air & $1665 b$ & 62 & River Sediment & 1645 \\
\hline 59 & Propane in Air & $1666 \mathrm{~b}$ & 105 & River Sediment, Environmental & $4350 \mathrm{~B}$ \\
\hline 59 & Propane in Air & $1667 \mathrm{~b}$ & & Radioactivity & \\
\hline 59 & Propane in Air & $1668 \mathrm{~b}$ & 105 & Rocky Flats Soil Number 1, & 4353 \\
\hline 59 & Propane in Air & $1669 b$ & & Environmental Radioactivity & \\
\hline \multirow[t]{2}{*}{60} & Propane in Nitrogen (Mobile Source & 2643 & 57,93 & Rubidium Melting Point & 1969 \\
\hline & Emission Gas Standard) & & 71 & Rutile Ore & 670 \\
\hline 60 & $\begin{array}{l}\text { Propane in Nitrogen (Mobile Source } \\
\text { Emission Gas Standard) }\end{array}$ & 2644 & 84 & $\begin{array}{l}\text { Scanning Electron Microscope } \\
\text { Magnification Standard }\end{array}$ & $484 c$ \\
\hline 60 & $\begin{array}{l}\text { Propane in Nitrogen (Mobile Source } \\
\text { Emission Gas Standard) }\end{array}$ & 2645 & 84 & $\begin{array}{l}\text { Scanning Electron Microscope } \\
\text { Performance Standard }\end{array}$ & 2069 \\
\hline 60 & $\begin{array}{l}\text { Propane in Nitrogen (Mobile Source } \\
\text { Emission Gas Standard) }\end{array}$ & 2646 & 112 & $\begin{array}{l}\text { Secondary Standard Flexible Disk } \\
\text { Cartridge (Computer Amplitude }\end{array}$ & 3210 \\
\hline \multirow[t]{2}{*}{60} & Propane in Nitrogen (Mobile Source & 2647 & & Reference) & \\
\hline & Emission Gas Standard) & & 112 & Secondary Standard Magnetic Tape & 3200 \\
\hline 60 & $\begin{array}{l}\text { Propane in Nitrogen (Mobile Source } \\
\text { Emission Gas Standard) }\end{array}$ & 2648 & 112 & $\begin{array}{l}\text { Secondary Standard Magnetic Tape } \\
\text { Cassette }\end{array}$ & 1600 \\
\hline 60 & $\begin{array}{l}\text { Propane in Nitrogen (Mobile Source } \\
\text { Emission Gas Standard) }\end{array}$ & 2649 & 112 & $\begin{array}{l}\text { Secondary Standard Magnetic Tape } \\
\text { Cartridge (Computer Amplitude }\end{array}$ & 3216 \\
\hline \multirow[t]{2}{*}{60} & Propane in Nitrogen (Mobile Source & 2650 & \multirow{3}{*}{98} & Reference) & \multirow{3}{*}{2023} \\
\hline & Emission Gas Standard) & & & Second Surface Aluminum Mirror for & \\
\hline 60 & Propane in Nitrogen and Oxygen & 2651 & & Specular Reflectance & \\
\hline
\end{tabular}

60 Propane in Nitrogen and Oxygen (Mobile Source Emission Gas Standard)

57,96 Quartz Cuvette for Spectrophotometry 932

93 Quartz for Hydrofluoric Acid 1654 Solution Calorimetry 


\begin{tabular}{|c|c|c|c|c|c|}
\hline Page & Name & SRM & Page & Name & SRM \\
\hline \multirow[t]{2}{*}{98} & \multirow{2}{*}{$\begin{array}{l}\text { Second Surface Aluminum Mirror for } \\
\text { Specular Reflectance }\end{array}$} & \multirow[t]{2}{*}{2024} & 74 & Soda-Lime Sheet Glass & 1831 \\
\hline & & & 87 & Soda-Lime Silica Glass & 622 \\
\hline \multirow[t]{2}{*}{98} & \multirow{2}{*}{$\begin{array}{l}\text { Second Surface Aluminum Mirror with } \\
\text { Wedge for Specular Reflectance }\end{array}$} & \multirow[t]{2}{*}{2025} & 88 & Soda-Lime Silica Glass & 710 \\
\hline & & & 89 & Soda-Lime Silica Glass for Liquidus & 773 \\
\hline 31 & Selenium-Bearing Steel & $1170 \mathrm{~b}$ & & Temperature & \\
\hline 105 & Selenium-75 Radioactivity Standard & 4409L-D & 83 & Sodium Bicarbonate & $191 \mathrm{a}$ \\
\hline 39 & Sheet Brass & $37 \mathrm{E}$ & 84 & Sodium Bicarbonate & 2191 \\
\hline 73 & Silica Brick & 198 & 83 & Sodium Carbonate & $192 \mathrm{a}$ \\
\hline 73 & Silica Brick & 199 & 84 & Sodium Carbonate & 2192 \\
\hline 39 & Silicon-Aluminum Alloy & $87 \mathrm{a}$ & 84 & Sodium Chloride & 2201 \\
\hline 39 & Silicon Bronze & $158 \mathrm{~A}$ & 56 & Sodium Chloride (Clinical Standard) & 919 \\
\hline 90 & Silicon Density Standard & 1840 & 67 & Sodium Cyclohexanebutyrate & $1069 b$ \\
\hline 90 & Silicon Density Standard & 1841 & 55 & Sodium Oxalate Reductometric & $40 \mathrm{~h}$ \\
\hline 35 & Silicon Metal & $57 \mathrm{a}$ & & Standard & \\
\hline \multirow[t]{2}{*}{107} & \multirow{2}{*}{$\begin{array}{l}\text { Silicon Powder, Spacing Standard } \\
\text { for X-ray Diffraction }\end{array}$} & \multirow[t]{2}{*}{$640 \mathrm{a}$} & 56 & Sodium Pyruvate & 910 \\
\hline & & & 83 & Sodium Tetraborate Decahydrate & $187 \mathrm{~b}$ \\
\hline \multirow[t]{2}{*}{108} & Silicon Power Device Level & \multirow[t]{2}{*}{1522} & & (Borax) & \\
\hline & Resistivity Standard & & 42 & Solder & $127 b$ \\
\hline \multirow{2}{*}{108} & \multirow{2}{*}{$\begin{array}{l}\text { Silicon Resistivity Standard for Eddy } \\
\text { Current Testers }\end{array}$} & \multirow{2}{*}{1523} & 49 & Solder & 1131 \\
\hline & & & 81 & Special Nuclear Container DOT 6M, & 9940 \\
\hline 67 & Silver 2-Ethylhexanoate & $1077 \mathrm{a}$ & & 15 gal. & \\
\hline 95 & Silver-Gold Thermocouple Wire & 733 & 81 & Special Nuclear Container, 55 gal. & 9941 \\
\hline 94 & Silver, Vapor Pressure & 748 & 81 & Special Nuclear Container Type A, & 9942 \\
\hline \multirow[t]{2}{*}{94,108} & \multirow{2}{*}{$\begin{array}{l}\text { Sintered and Arc-Cast Tungsten, } \\
\text { Thermal Conductivity and } \\
\text { Electrical Resistivity }\end{array}$} & \multirow[t]{2}{*}{1465} & & 10 gal. & \\
\hline & & & 81 & $\begin{array}{l}\text { Special Nuclear Container, Type A, } \\
55 \text { gal. }\end{array}$ & 9943 \\
\hline \multirow[t]{2}{*}{94,108} & \multirow{2}{*}{$\begin{array}{l}\text { Sintered and Arc-Cast Tungsten, } \\
\text { Thermal Conductivity and } \\
\text { Electrical Resistivity }\end{array}$} & \multirow[t]{2}{*}{1466} & 106 & Special Nuclear Material Package & 9910 \\
\hline & & & 26 & $\begin{array}{l}\text { Spectrographic Ingot Iron and } \\
\text { Low-Alloy Steel Standard (Rod) }\end{array}$ & 461 \\
\hline \multirow[t]{2}{*}{94,108} & \multirow{2}{*}{$\begin{array}{l}\text { Sintered and Arc-Cast Tungsten, } \\
\text { Thermal Conductivity and } \\
\text { Electrical Resistivity }\end{array}$} & \multirow[t]{2}{*}{1467} & 26 & $\begin{array}{l}\text { Spectrographic Ingot Iron and } \\
\text { Low-Alloy Steel Standard (Rod) }\end{array}$ & 462 \\
\hline & & & 26 & Spectrographic Ingot Iron and & 463 \\
\hline \multirow[t]{2}{*}{94,108} & Sintered and Arc-Cast Tungsten, & 1468 & & Low-Alloy Steel Standard (Rod) & \\
\hline & $\begin{array}{l}\text { Thermal Conductivity and } \\
\text { Electrical Resistivity }\end{array}$ & & 26 & $\begin{array}{l}\text { Spectrographic Ingot Iron and } \\
\text { Low-Alloy Steel Standard (Rod) }\end{array}$ & 464 \\
\hline 115 & $\begin{array}{l}\text { Smoke Density Chamber Standard } \\
\text { (Flaming Exposure Condition) }\end{array}$ & $1007 \mathrm{a}$ & 26 & $\begin{array}{l}\text { Spectrographic Ingot Iron and } \\
\text { Low-Alloy Steel Standard (Rod) }\end{array}$ & 465 \\
\hline 115 & $\begin{array}{l}\text { Smoke Density Chamber Standard } \\
\text { (Non-flaming Exposure Condition) }\end{array}$ & $1006 b$ & 26 & $\begin{array}{l}\text { Spectrographic Ingot Iron and } \\
\text { Low-Alloy Steel Standard (Rod) }\end{array}$ & 466 \\
\hline 74 & Soda-Lime Container Glass & 621 & 26 & Spectrographic Ingot Iron and & 467 \\
\hline 74 & Soda-Lime Flat Glass & 620 & & Low-Alloy Steel Standard (Rod) & \\
\hline 74 & Soda-Lime Float Glass & 1830 & 26 & Spectrographic Ingot Iron and & 468 \\
\hline 90 & Soda-Lime Glass & 1826 & & Low-Alloy Steel Standard (Rod) & \\
\hline 74 & Soda-Lime Glass Powder & 92 & 26 & $\begin{array}{l}\text { Spectrograhic Ingot Iron and } \\
\text { Low-Alloy Steel Standard }\end{array}$ & 1166 \\
\hline & & & 30 & $\begin{array}{l}\text { Spectrographic Stainless Steel } \\
\text { Standard }\end{array}$ & 442 \\
\hline & & & 30 & $\begin{array}{l}\text { Spectrographic Stainless Steel } \\
\text { Standard }\end{array}$ & 443 \\
\hline & & & 30 & $\begin{array}{l}\text { Spectrographic Stainless Steel } \\
\text { Standard }\end{array}$ & 444 \\
\hline & & & 31 & $\begin{array}{l}\text { Spectrographic Stainless Steel } \\
\text { Standard (Disc) }\end{array}$ & D849 \\
\hline & & & 31 & $\begin{array}{l}\text { Spectrographic Stainless Steel } \\
\text { Standard (Disc) }\end{array}$ & $\mathrm{D} 850$ \\
\hline & & & 31 & $\begin{array}{l}\text { Spectrographic Stainless Steel } \\
\text { Standard (Group II) }\end{array}$ & 445 \\
\hline
\end{tabular}




\section{Page}

Name

31

31

31

31

31

31

31

25

25

25

25

25

25

25

25

25

25

25

34

34

34

34

34

34

34

34

34 Standard (Rod)

Standard (Rod) (Disc) (Disc) (Disc) Alloy A Alloy B Alloy C Alloy D Alloy E Alloy F
Spectrographic Stainless Steel Standard (Group II)

Spectrographic Stainless Steel Standard (Group II)

Spectrographic Stainless Steel Standard (Group II)

Spectrographic Stainless Steel Standard (Group II)

Spectrographic Stainless Steel Standard (Group II)

Spectrographic Stainless Steel

Spectrographic Stainless Steel

Spectrographic Steel Standard (Disc)

Spectrographic Steel Standard (Disc)

Spectrographic Steel Standard (Rod)

Spectrographic Steel Standard (Rod)

Spectrographic Steel Standard (Rod)

Spectrographic Steel Standard (Rod)

Spectrographic Steel Standard (Rod)

Spectrographic Steel Standard (Rod)

Spectrographic Steel Standard (Rod)

Spectrographic Steel Standard (Rod)

Spectrographic Steel Standard (Rod)

Spectrographic Steel Standard (Rod)

Spectrographic Tool Steel Standard

Spectrographic Tool Steel Standard

Spectrographic Tool Steel Standard

Spectrographic Tool Steel Standard

Spectrographic Tool Steel Standard

Spectrographic Tool Steel Standard

Spectrographic Tool Steel Standard

Spectrographic Tool Steel Standard

Spectrographic Tool Steel Standard

Spectrographic Tool Steel Standard

Spectrographic Tool Steel Standard

Spectrographic Zinc-Base Die-Casting

Spectrographic Zinc-Base Die-Casting

Spectrographic Zinc-Base Die-Casting

Spectrographic Zinc-Base Die-Casting

Spectrographic Zinc-Base Die-Casting

Spectrographic Zinc-Base Die-Casting

Spectrographic Zinc Spelter Standard Spectroscopic Titanium-Base Standard Spectroscopic Titanium-Base Standard Spectroscopic Titanium-Base Standard
SRM

Page

Name

SRM

$44650 \quad$ Spectroscopic Titanium-Base Standard

$849 \quad 108$

$850 \quad 57$

D803a $\quad 22$

D807a 22

$803 a \quad 22$

$804 a \quad 31$

$805 a \quad 22$

$807 a \quad 31$

$808 \mathrm{a} \quad 31$

$809 a \quad 31$

$817 \mathrm{~b} \quad 31$

$820 \mathrm{a} \quad 31$

$821 \quad 31$

$827 \quad 31$

$436 \quad 31$

$437 \quad 31$

$438 \quad 31$

$439 \quad 31$

$440 \quad 107$

441

837

840

D837

26

D840 67

D841 106

111

625

626

627

627

628

629

630

631

641

642

643
Spectroscopic Titanium-Base Standard

Spectroscopic Titanium-Base Standard

Spheroidized Iron Carbide in Ferrite

Spreading Resistance Calibration

(100) n-Type Silicon

Spreading Resistance Calibration (100) p-Type Silicon

Spreading Resistance Calibration

(111) n-Type Silicon

Spreading Resistance Calibration

(11) p-Type Silicon

644

645

646

493

2529

2528

2527

2526

1590

$121 \mathrm{~d}$

$123 \mathrm{c}$

$160 \mathrm{~b}$

367

1267

$73 \mathrm{c}$

C1151

$1151 \mathrm{a}$

C1152

$1152 \mathrm{a}$

C1153

$1153 \mathrm{a}$

C1154

$1154 \mathrm{a}$

1155

1172

1171

1890

Stainless Steel for Pitting or Crevice Corrosion

Stainless Steel Thermal Expansion

Stearic Acid Rubber Compound

Steel (AISI 1211)

Steel (Lead-Bearing)

Strontium Cyclohexanebutyrate

Strontium-85 Radioactivity Standard

Strontium-89 Radioactivity Standard

Styrene-butadiene Rubber (Type 1500)

Succinonitrile Freezing Point

Sucrose

Sulfate and Nitrate on Filter Media

738

$372 \mathrm{~h}$

368

$1169 \mathrm{~b}$

1070a

4403L-B

4945D

$386 \mathrm{~h}$

1970

$17 \mathrm{c}$

2673

$1661 \mathrm{a}$

$1662 \mathrm{a}$

$1663 a$

$1664 \mathrm{a}$

1693

1694 
Page

Name

59

61

61

61

65

65

65

65 ( $2 \mathrm{~cm}$ tube) ( $5 \mathrm{~cm}$ tube) (10 $\mathrm{cm}$ tube)

Sulfur in Coal

Sulfur in Coal

Sulfur in Coal

Sulfur in Coal Point Device Point Device

Synthetic Sapphire Standard Batt Board

Tin, Freezing Point Standard Standard

Titanium Alloy

Titanium-Base Alloy

Titanium-Base Alloy
Sulfur Dioxide in Nitrogen

Sulfur Dioxide Permeation Tube

Sulfur Dioxide Permeation Tube

Sulfur Dioxide Permeation Tube

Sulfur in Residual Fuel Oil

Sulfur in Residual Fuel Oil

Sulfur in Residual Fuel Oil

Sulfur in Residual Fuel Oil

Sulfur in Residual Fuel Oil

Sulfur in Residual Fuel Oil

Sulfur Rubber Compound

Superconductive Thermometric Fixed

Superconductive Thermometric Fixed

Surface Flammability Standard

Technetium-99 Radioactivity Standard

Technetium-99m Radioactivity

Tetrachloroethylene in Nitrogen

Thallium-201 Radioactivity Standard

Thermal Resistance, Fibrous Glass

Thermal Resistance, Fibrous Glass

Thorium-228, Thallium-208 Gamma-ray Point-Source Standard

Tin-Base Bearing Metal

Tin-113-Indium-113m Radioactivity

Tin. $121 \mathrm{~m}$ Point-Source Gamma-ray Emission-Rate Standard

Tin, Secondary Freezing Point

\section{SRM}

1696

1627

1626

1625

2682

2683

2684

2685

1619

$1620 \mathrm{a}$

$1621 \mathrm{~b}$

$1622 \mathrm{~b}$

$1623 \mathrm{a}$

$1624 \mathrm{a}$

$371 \mathrm{~g}$

$767 \mathrm{a}$

768

$1002 \mathrm{c}$

720

4288

$4410 \mathrm{H}$.I

1808

4404L-F

1451

$1450 \mathrm{~b}$

$4206 \mathrm{C}$

54D

741

4402L.C

4264B

$42 \mathrm{~g}$

$654 \mathrm{a}$

$173 \mathrm{~b}$

176

\section{Page}

Name

SRM

44

44

44

72

99

57

23

34

107

43

43

43

76

76

76

76
76

76

76

76

76

62

62

62

62

62

61

$90,93,99$

57

55

93

56,83

56,83

67

67

67

67

67
74

23

71

94

54

Titanium-Base Alloy (Unalloyed)

650

Titanium-Base Alloy (Unalloyed)

Titanium-Base Alloy (Unalloyed)

Titanium Dioxide

Toluene

Tomato Leaves

Tool Steel (AISI M2)

Tool Steel (AISI M2)

Tool Steel Abrasive Wear Standard

Tracealloy (Nickel-Base High-Temperature Alloy)

Tracealloy (Nickel-Base

High-Temperature Alloy)

Tracealloy (Nickel-Base

High-Temperature Alloy)

Trace Elements in a Glass Matrix

Trace Elements in a Glass Matrix

Trace Elements in a Glass Matrix

Trace Elements in a Glass Matrix

Trace Elements in a Glass Matrix

Trace Elements in a Glass Matrix

Trace Elements in a Glass Matrix

Trace Elements in a Glass Matrix

Trace Elements in Coal (Bituminous)

Trace Elements in Coal (Subbituminous

Trace Elements in Coal Fly Ash

Trace Elements in Fuel Oil

Trace Elements in Water

Trace Mercury in Coal

651

652

$154 b$

$211 \mathrm{c}$

1573

$132 \mathrm{~b}$

1157

1857

897

898

899

610

611

612

613

614

615

616

617

$1632 \mathrm{a}$

1635

$1633 a$

$1634 \mathrm{a}$

$1643 \mathrm{a}$

1630

$217 \mathrm{c}$

1595

$723 \mathrm{a}$

$724 a$

922

923

$1078 \mathrm{~b}$

$1079 \mathrm{~b}$

$1071 \mathrm{~b}$

$276 \mathrm{a}$

$50 \mathrm{c}$

277

782

480

Tungsten-20\% Molybdenum Alloy Electron Microprobe Standard

Tungsten Thermal Expansion

Unalloyed Copper

Unalloyed Copper, $\mathrm{Cu}$ "O'

737

1034

393

Unalloyed Copper, Cu IV

Unalloyed Copper, Cu XI

Unalloyed Copper, Cu I (Chip)

Unalloyed Copper, Cu II (Chip)

Unalloyed Copper, Cu III (Chip)

Unalloyed Copper, Cu V (Chip)

Unalloyed Copper, Cu VI (Chip)

Unalloyed Copper, Cu VII (Chip)

Unalloyed Copper, Cu I (Rod) 


\begin{tabular}{|c|c|c|c|c|c|}
\hline Page & Name & SRM & Page & Name & SRM \\
\hline 48 & Unalloyed Copper, Cu II (Rod) & 495 & \multirow[t]{2}{*}{68} & \multirow{2}{*}{$\begin{array}{l}\text { Wear-Metals in Lubricating Oil } \\
(300 \mathrm{ppm})\end{array}$} & \multirow[t]{2}{*}{1085} \\
\hline 48 & Unalloyed Copper, Cu III (Rod) & 496 & & & \\
\hline 48 & Unalloyed Copper, Cu V (Rod) & 498 & 57 & Wheat Flour & 1567 \\
\hline 48 & Unalloyed Copper, Cu VI (Rod) & 499 & 36 & White Cast Iron & 338 \\
\hline 48 & Unalloyed Copper, Cu VII (Rod) & 500 & 38 & White Cast Iron (Disc) & 1145 \\
\hline 51 & Unalloyed Titanium & 354 & 38 & White Cast Iron (Disc) & 1146 \\
\hline \multirow[t]{2}{*}{78} & \multirow{2}{*}{$\begin{array}{l}\text { Uranium Isotopic Standard (Nominally } \\
\text { depleted to } 0.02 \% \text { ) }\end{array}$} & \multirow[t]{2}{*}{ U-0002 } & 38 & White Cast Iron (Disc) & 1150 \\
\hline & & & 98 & White Ceramic Tile for Directional & \multirow[t]{2}{*}{$2019 b$} \\
\hline 78 & Uranium Isotopic Standard & U.005a & & Hemispherical Reflectance & \\
\hline 78 & $\begin{array}{l}\text { Uranium Isotopic Standard } \\
\text { (Nominally } 1 \% \text { Enriched) }\end{array}$ & U-010 & 98 & $\begin{array}{l}\text { White Ceramic Tile for Directional } \\
\text { Hemispherical Reflectance }\end{array}$ & 2020 \\
\hline \multirow[t]{2}{*}{78} & \multirow{2}{*}{$\begin{array}{l}\text { Uranium Isotopic Standard } \\
\text { (Nominally } 1.5 \% \text { Enriched) }\end{array}$} & \multirow[t]{2}{*}{ U.015 } & 36 & White Iron & \multirow{4}{*}{$\begin{array}{l}3 d \\
2015\end{array}$} \\
\hline & & & 98 & White Opan Glass Diffuse Spectral & \\
\hline 78 & Uranium Isotopic Standard & U-020 & & Reflectance Standard for the & \\
\hline 78 & Uranium Isotopic Standard & U-030a & & Visible Spectrum & \\
\hline 78 & $\begin{array}{l}\text { Uranium Isotopic Standard } \\
\text { (Nominally 5\% Enriched) }\end{array}$ & U-050 & 103 & $\begin{array}{l}\text { Xenon- } 127 \text { Gaseous Radioactivity } \\
\text { Standard }\end{array}$ & $4309 \mathrm{G}$ \\
\hline 78 & $\begin{array}{l}\text { Uranium Isotopic Standard } \\
\text { (Nominally 10\% Enriched) }\end{array}$ & $\mathrm{U} \cdot 100$ & 103 & $\begin{array}{l}\text { Xenon-133 Gaseous Radioactivity } \\
\text { Standard }\end{array}$ & $4307 \mathrm{I}$ \\
\hline 78 & $\begin{array}{l}\text { Uranium Isotopic Standard } \\
\text { (Nominally } 15 \% \text { Enriched) }\end{array}$ & U. 150 & 103,106 & $\begin{array}{l}\text { Xenon-133 Gaseous Radioactivity } \\
\text { Standard }\end{array}$ & $4415 \mathrm{~L}-\mathrm{I}$ \\
\hline 78 & $\begin{array}{l}\text { Uranium Isotopic Standard } \\
\text { (Nominally 20\% Enriched) }\end{array}$ & U-200 & 103 & $\begin{array}{l}\text { Xenon-133, Xenon-137, Krypton- } 85 \\
\text { Mixed Gaseous Radioactivity }\end{array}$ & $4310 B$ \\
\hline \multirow[t]{2}{*}{78} & \multirow{2}{*}{$\begin{array}{l}\text { Uranium Isotopic Standard } \\
\text { (Nominally 35\% Enriched) }\end{array}$} & \multirow[t]{2}{*}{ U-350 } & & Standard & \\
\hline & & & 115 & X-ray Film Step Tablet & 1001 \\
\hline 78 & $\begin{array}{l}\text { Uranium Isotopic Standard } \\
\text { (Nominally } 50 \% \text { Enriched) }\end{array}$ & U. 500 & 107 & $\begin{array}{l}\text { X-ray Powder Diffraction Intensity } \\
\text { Standard }\end{array}$ & 674 \\
\hline 78 & $\begin{array}{l}\text { Uranium Isotopic Standard } \\
\text { (Nominally } 75 \% \text { Enriched) }\end{array}$ & U-750 & 107 & $\begin{array}{l}\text { X-ray Powder Diffraction (Mica) } \\
\text { Low } 2 \text { Theta }\end{array}$ & 675 \\
\hline \multirow[t]{2}{*}{78} & \multirow{2}{*}{$\begin{array}{l}\text { Uranium Isotopic Standard } \\
\text { (Nominally } 80 \% \text { Enriched) }\end{array}$} & \multirow[t]{2}{*}{$\mathrm{U}-800$} & 106 & Ytterbium-169 Radioactivity Standard & 4419L-B \\
\hline & & & 45 & Zinc-Base Alloy (Die Casting) & $94 c$ \\
\hline \multirow[t]{2}{*}{78} & \multirow{2}{*}{$\begin{array}{l}\text { Uranium Isotopic Standard } \\
\text { (Nominally } 85 \% \text { Enriched) }\end{array}$} & \multirow[t]{2}{*}{ U.850 } & 71 & Zinc Concentrates & $113 a$ \\
\hline & & & 71 & Zinc Concentrates & 329 \\
\hline \multirow[t]{2}{*}{78} & Uranium Isotopic Standard & U-900 & 67 & Zinc Cyclohexanebutyrate & $1073 b$ \\
\hline & (Nominally $90 \%$ Enriched) & & 92 & Zinc, Freezing Point & 740 \\
\hline 78 & Uranium Isotopic Standard & U.930 & 92 & Zinc, Freezing Point Standard & $43 h$ \\
\hline & (Nominally 93\% Enriched) & & 52 & Zinc Metal & 683 \\
\hline 78 & Uranium Isotopic Standard & U-970 & 111 & Zinc Oxide Rubber Compound & $370 \mathrm{e}$ \\
\hline & (Nominally 97\% Enriched) & & 45 & Zircaloy-2 & $360 \mathrm{a}$ \\
\hline 55,78 & Uranium Metal & 960 & 51 & Zircaloy-4 Metal & 1237 \\
\hline 55,78 & Uranium Oxide & $950 \mathrm{~b}$ & 51 & Zircaloy-4 Metal & 1238 \\
\hline 78 & Uranium Oxide & 969 & 51 & Zircaloy-4 Metal & 1239 \\
\hline 78 & $\begin{array}{l}\text { Uranium-233 Spike Assay and } \\
\text { Isotopic Solution Standard }\end{array}$ & 995 & 93 & $\begin{array}{l}\text { Zirconium-Barium Chromate } \\
\text { Formulation for Heat-Source }\end{array}$ & 1651 \\
\hline 78 & Uranium-235 Spike Assay and & 993 & & Powder Calorimetry & \\
\hline & Isotopic Solution Standard & & 93 & Zirconium-Barium Chromate & 1652 \\
\hline 64 & Urban Dust/Organics & 1649 & & Formulation for Heat-Source & \\
\hline 62 & Urban Particulate Matter & 1648 & & Powder Calorimetry & \\
\hline 56 & Urea & $912 \mathrm{a}$ & 93 & Zirconium-Barium Chromate & 1653 \\
\hline 56 & Urea & 2141 & & Formulation for Heat-Source & \\
\hline 56 & Urea & 2152 & & Powder Calorimetry & \\
\hline 56 & Uric Acid & 913 & 51 & Zirconium Metal & 1234 \\
\hline 61 & Vanadium and Nickel in Residual & 1618 & 51 & Zirconium Metal & 1235 \\
\hline & Fuel Oil & & 51 & Zirconium Metal & 1236 \\
\hline 62,79 & Vanadium in Curde Oil & 8505 & & & \\
\hline 104 & $\begin{array}{l}\text { Vanadium-49 Low-Energy Photon } \\
\text { Standard }\end{array}$ & 4266 & & & \\
\hline 42 & Waspaloy & 349 & & & \\
\hline 68 & Wear-Metals in Lubricating Oil & 1084 & & & \\
\hline
\end{tabular}





\section{Numerical Index to Standard Reference Material Certificates}

The Certificate date listed is the current version of the Certificate. Those dates followed by the letter "P" indicate that it is a Provisional Certificate.

In general, Provisional Certificates are issued for Standard Reference Materials before all of the values have been certified, but after a sufficient number of values are certified so that the material is a valuable standard for the intended purpose. As additional values are certified, the Provisional Certificate may be revised and when and if all of the value are certified, the final Certificate is issued.

SRM purchasers whose Certificate shows an earlier date then listed below may obtain the current version of the Certificate from the Office of Standard Reference Materials, Room B311, Chemistry Building, National Bureau of Standards, Washington, DC 20234.

One or more of the following letters apply to materials where no date is listed.
A. Individually Certified.
B. The materials is issued with "Instructions for Use" in lieu of a Certificate.
C. This material is not certified; refer to page reference for details.
D. Material is in preparation.
E. Research Material: Issued with a "Report of Investigation."
F. Special Reference Material: Information provided, but not certified by NBS.
G. Set of SRM's: Issued with Certificates for the individual SRM's.
H. Not individually issued; part of a set.

\begin{tabular}{|c|c|c|c|c|c|}
\hline Page & SRM & Certificate Date & Page & SRM & Certificate Date \\
\hline 72 & $1 \mathrm{c}$ & $12-14-78$ & 92 & $42 \mathrm{~g}$ & $7.18-72$ \\
\hline 36 & $3 \mathrm{~d}$ & $4-2.79$ & 92 & $43 \mathrm{~h}$ & $8-15-73$ \\
\hline 36 & $4 \mathrm{k}$ & $5.27-76$ & 92 & $44 f$ & $4-5.73$ \\
\hline 36 & $5 \mathrm{~L}$ & $11-9.70$ & 92 & $45 d$ & $12-6-71$ \\
\hline 36 & $6 g$ & $11 \cdot 9.70$ & 92 & $49 \mathrm{e}$ & $12-6-71$ \\
\hline 36 & $7 \mathrm{G}$ & $10-5-59$ & 23 & $50 c$ & $6-25-57$ \\
\hline 19 & $8 \mathrm{j}$ & $4 \cdot 10-72$ & 42 & $53 e$ & $1-20-70$ \\
\hline 19 & $11 \mathrm{~h}$ & $4-30-74$ & 44 & $54 \mathrm{D}$ & $9-20-57$ \\
\hline 19 & $12 \mathrm{H}$ & 3.7 .66 & 35 & $57 \mathrm{a}$ & $12 \cdot 30 \cdot 80$ \\
\hline 19 & $13 \mathrm{~g}$ & $4-30.74$ & 35 & $58 \mathrm{a}$ & $4-25-78$ \\
\hline 19 & $14 \mathrm{f}$ & $2-24-81$ & 35 & $59 a$ & $11-6.69$ \\
\hline 19 & $15 \mathrm{~g}$ & $4 \cdot 30-74$ & 35 & $64 c$ & $8-24-77$ \\
\hline 19 & $16 f$ & $3-9-83$ & 35 & $68 \mathrm{c}$ & $8-15-79$ \\
\hline 55,100 & $17 \mathrm{c}$ & 4-1-81 & 70 & $69 b$ & $8.24-79$ \\
\hline 19 & $19 \mathrm{G}$ & $9.30-64$ & 72 & $70 \mathrm{a}$ & $10-10-81$ \\
\hline 19 & $20 \mathrm{~g}$ & $10-23-70$ & 35 & 71 & 2.1 .29 \\
\hline 71 & $25 \mathrm{~d}$ & $\mathrm{D}$ & 20 & $72 \mathrm{~g}$ & $6-4-81$ \\
\hline 70 & $27 f$ & $5.31-77$ & 22 & $73 c$ & $7-13.66$ \\
\hline 20 & $30 \mathrm{f}$ & $6-5-79$ & 72 & $76 a$ & $4-5-77$ \\
\hline 20 & $32 \mathrm{E}$ & $4-5.57$ & 72 & $77 a$ & $4-5-77$ \\
\hline 20 & $33 d$ & $6-10-55$ & 72 & $78 a$ & 4.5 .77 \\
\hline 20 & $36 b$ & 7.18 .69 & 69 & $79 a$ & $1-8.80$ \\
\hline 39 & $37 \mathrm{E}$ & $8-28-58$ & 72 & $81 \mathrm{a}$ & $1-78$ \\
\hline 93 & $39 \mathrm{i}$ & $7-15.68$ & & & \\
\hline 55 & $40 \mathrm{~h}$ & $5-4-82$ & & & \\
\hline 55,100 & $41 b$ & $4-10-75$ & & & \\
\hline
\end{tabular}




\begin{tabular}{|c|c|}
\hline Page & SRM \\
\hline 36 & $82 b$ \\
\hline 55 & $83 \mathrm{~d}$ \\
\hline 55 & $84 j$ \\
\hline 39 & $85 \mathrm{~B}$ \\
\hline 39 & $87 a$ \\
\hline 72 & $88 \mathrm{a}$ \\
\hline 74 & 89 \\
\hline 35 & 90 \\
\hline 74 & 91 \\
\hline 74 & 92 \\
\hline 74 & $93 a$ \\
\hline 45 & $94 \mathrm{c}$ \\
\hline 72 & $97 \mathrm{a}$ \\
\hline 72 & $98 \mathrm{a}$ \\
\hline 72 & $99 a$ \\
\hline 20 & $100 \mathrm{~B}$ \\
\hline 23 & $101 \mathrm{f}$ \\
\hline 73 & $103 a$ \\
\hline 20 & 105 \\
\hline 20 & $106 \mathrm{~B}$ \\
\hline 36 & $107 \mathrm{C}$ \\
\hline 71 & $113 a$ \\
\hline 113 & $11,4 \mathrm{n}$ \\
\hline 36 & $115 \mathrm{~A}$ \\
\hline 68,71 & $120 \mathrm{~b}$ \\
\hline 22 & $121 d$ \\
\hline 36 & $122 \mathrm{~h}$ \\
\hline 22 & $123 \mathrm{c}$ \\
\hline 20 & $125 \mathrm{~b}$ \\
\hline 22 & $126 \mathrm{c}$ \\
\hline 42 & $127 \mathrm{~b}$ \\
\hline 20 & $129 \mathrm{c}$ \\
\hline 20 & $131 \mathrm{c}$ \\
\hline 23 & $132 b$ \\
\hline 22 & $133 \mathrm{~B}$ \\
\hline 23 & $134 \mathrm{~A}$ \\
\hline 55 & $136 d$ \\
\hline 20 & $139 b$ \\
\hline 55 & $140 \mathrm{~b}$ \\
\hline 55 & $141 \mathrm{c}$ \\
\hline 55 & 142 \\
\hline 55 & $143 c^{\circ}$ \\
\hline 55 & 148 \\
\hline 19 & $152 \mathrm{~A}$ \\
\hline 23 & $153 \mathrm{~A}$ \\
\hline 72 & $154 \mathrm{~b}$ \\
\hline 20 & 155 \\
\hline 39 & $158 \mathrm{~A}$ \\
\hline 22 & $160 \mathrm{~b}$ \\
\hline 23 & 163 \\
\hline 72 & $165 a$ \\
\hline 22 & $166 c$ \\
\hline
\end{tabular}

\begin{tabular}{|c|c|c|}
\hline Certificate Date & Page & SRM \\
\hline $4-12.66$ & 42 & 171 \\
\hline $3 \cdot 1-82$ & 44 & $173 b$ \\
\hline $10-11.78$ & 44 & 176 \\
\hline $5-9-57$ & 19 & 178 \\
\hline $10-10-81$ & 20 & 179 \\
\hline $7.22-82$ & 69 & 180 \\
\hline $8-27-32$ & 69 & 181 \\
\hline $10-1-28$ & 69 & 182 \\
\hline $10-28-82$ & 69 & 183 \\
\hline $3-11.82$ & 83 & $185 \mathrm{e}$ \\
\hline $8-31-73$ & 83 & $186 \mathrm{Ic}$ \\
\hline $8-15-73$ & 83 & $186 \mathrm{IIC}$ \\
\hline $10-8-69$ & 83 & $187 b$ \\
\hline $10-8-69$ & 83 & 188 \\
\hline $10-10-81$ & 83 & 189 \\
\hline 8-18-59 & 83 & $191 \mathrm{a}$ \\
\hline 5.19 .70 & 83 & $192 \mathrm{a}$ \\
\hline $9-28-62$ & 68 & 193 \\
\hline $10-10-81$ & 68 & 194 \\
\hline $3-24-61$ & 35 & 195 \\
\hline $5-16-83$ & 35 & 196 \\
\hline $12-29-75$ & 73 & 198 \\
\hline $2 \cdot 26-82$ & 73 & 199 \\
\hline 4-19-62 & 68 & 200 \\
\hline $7.31-79$ & 99 & $211 \mathrm{c}$ \\
\hline $8-31-81$ & $90,93,99$ & $217 \mathrm{c}$ \\
\hline $4-28-83$ & 74 & $276 a$ \\
\hline $10.1-81$ & 71 & 277 \\
\hline $2-24-82$ & 72 & 278 \\
\hline $12-30.77$ & 20 & 291 \\
\hline $10-1-81$ & 20 & 293 \\
\hline 8.7 .73 & 71 & 329 \\
\hline $6-2.77$ & 69 & 330 \\
\hline $8-16-73$ & 69 & 331 \\
\hline $8 \cdot 12.81$ & 69 & 332 \\
\hline $5 \cdot 6.57$ & 69 & 333 \\
\hline $8 \cdot 1.81$ & 36 & 334 \\
\hline $5-17.78$ & 19 & 335 \\
\hline $\mathrm{D}$ & 19 & 337 \\
\hline $9-27.76$ & 36 & 338 \\
\hline $7-9-69$ & 22 & 339 \\
\hline 9.27 .76 & 35 & 340 \\
\hline $12-22-70$ & 36 & 341 \\
\hline $10-11-65$ & 36 & $342 \mathrm{a}$ \\
\hline $1-5-60$ & 22 & 344 \\
\hline $5-16-73$ & 22 & 345 \\
\hline $10-1-46$ & 22 & 348 \\
\hline $8-8-61$ & 42 & 349 \\
\hline $8-31-81$ & 55 & $350 \mathrm{a}$ \\
\hline 1.11 .68 & 51 & $352 b$ \\
\hline $10-16.78$ & 51 & 354 \\
\hline \multirow{10}{*}{$3-17-70$} & 51 & 355 \\
\hline & 51 & 357 \\
\hline & 51 & 358 \\
\hline & 45 & $360 \mathrm{a}$ \\
\hline & 20 & 361 \\
\hline & 20 & 362 \\
\hline & 20 & 363 \\
\hline & 20 & 364 \\
\hline & 36 & 365 \\
\hline & 22 & 367 \\
\hline
\end{tabular}

Certificate Date

11-27.51

D

10-1-81

7.-14.69

7-15-76

3-31-71

10-1-81

10-1-81

10-1.81

5.23-73

$9 \cdot 1.70$

$9-1-70$

9-9-70

1-10-64

1-10-64

5-11-83

5-11-83

11.14-74

1.8.74

4. $25-78$

11-9.70

1-6-60

1.6-60

8-7.74

D

D

5-30-80

1-24-78

8-19-81

10-1-75

3-27-75

12.29 .75

1-20-77

1-20-77

6-26-77

1-20-77

3-20-82

4.7-66

8-16-66

6-24-82

7.21.65

$11-9.70$

3-26-62

4-27-70

10-2-63

1-23-64

10-1-81

9-2-81

4-27-81

$8-22-83$

D

9.8-66

6-16-80

6-16-80

1-30-67

2-24-81

2-24-81

2.24. 81

2.24.81

2.24-81

7.21-77 


\begin{tabular}{|c|c|c|c|c|c|}
\hline Page & SRM & Certificate Date & Page & SRM & Certificate Date \\
\hline 19 & 368 & 1.1 .78 & 53 & 478 & $2.28-74$ \\
\hline 111 & $370 \mathrm{e}$ & $\mathrm{C}$ & 53 & $479 \mathrm{a}$ & $11-1-80$ \\
\hline 111 & $371 \mathrm{~g}$ & $\mathrm{C}$ & 54 & 480 & $11-22-68$ \\
\hline 111 & $372 \mathrm{~h}$ & $\mathrm{C}$ & 54 & 481 & $2-14-69$ \\
\hline 111 & $373 \mathrm{f}$ & $\mathrm{C}$ & 54 & 482 & $6-6-69$ \\
\hline 111 & $375 \mathrm{~g}$ & $\mathrm{C}$ & 54 & 483 & $6-11-71$ \\
\hline 111 & $378 \mathrm{~b}$ & $\mathrm{C}$ & 84 & $484 c$ & $\mathrm{D}$ \\
\hline 111 & $382 \mathrm{a}$ & $\mathrm{C}$ & 106 & $485 \mathrm{a}$ & $10-9.81$ \\
\hline 111 & $383 a$ & $\mathrm{C}$ & 106 & 486 & $3-6-81$ \\
\hline 111 & $384 d$ & $\mathrm{C}$ & 106 & 487 & 5.14 .82 \\
\hline 111 & $386 \mathrm{~h}$ & $3-10-75$ & 106 & 488 & $10-6-83$ \\
\hline 111 & $388 \mathrm{~L}$ & $12-3-82$ & 106 & 493 & $8-28-70$ \\
\hline 41 & 393 & $9-16-80$ & 48 & 494 & $1-20-78$ \\
\hline 41 & 394 & $7-4-76$ & 48 & 495 & $1-20-78$ \\
\hline 41 & 395 & 7.4 .76 & 48 & 496 & $1-20-78$ \\
\hline 41 & 396 & $7-4-76$ & 48 & 498 & $1-20-78$ \\
\hline 41 & 398 & $1-23-78$ & 48 & 499 & $1-20-78$ \\
\hline 41 & 399 & 1.23 .78 & 48 & 500 & $1-20-78$ \\
\hline 41 & 400 & $1-23-78$ & 76 & 607 & $5.21-73$ \\
\hline 25 & $404 a$ & $5-5-65$ & 76 & 610 & $1-4-82$ \\
\hline 25 & $405 a$ & $5-5-65$ & 76 & 611 & $1-4-82$ \\
\hline 25 & $407 a$ & $5-5-65$ & 76 & 612 & $1-4-82$ \\
\hline 25 & $408 a$ & $5-5-65$ & 76 & 613 & $1-4-82$ \\
\hline 25 & $409 b$ & $5-5.65$ & 76 & 614 & $1.4-82$ \\
\hline 25 & 413 & $5-5-65$ & 76 & 615 & $1-4-82$ \\
\hline 25 & 414 & $5-5-65$ & 76 & 616 & $1.4-82$ \\
\hline 25 & $417 \mathrm{a}$ & $5-5-65$ & 76 & 617 & $1.4-82$ \\
\hline 25 & $418 \mathrm{a}$ & $5-5-65$ & 74 & 620 & $1-13-82$ \\
\hline 25 & $420 \mathrm{a}$ & $5-5-65$ & 74 & 621 & $1-21-82$ \\
\hline 25 & 427 & 5.5 .65 & 87 & 622 & $3-19-76$ \\
\hline 34 & 436 & $9-22-59$ & 87 & 623 & $3-19-76$ \\
\hline 34 & 437 & $9-22-59$ & 88,109 & 624 & $10-25-77$ \\
\hline 34 & 438 & 9.22 .59 & 50 & 625 & $4-24-64$ \\
\hline 34 & 439 & $9-22.59$ & 50 & 626 & 4-24-64 \\
\hline 34 & 440 & $9-22-59$ & 50 & 627 & $4-24-64$ \\
\hline 34 & 441 & $9-22-59$ & 50 & 628 & $4-24-64$ \\
\hline 30 & 442 & $10-25-65$ & 50 & 629 & $4-24-64$ \\
\hline 30 & 443 & $10-25-65$ & 50 & 630 & $4-24-64$ \\
\hline 30 & 444 & $10-25-65$ & 50 & 631 & $11-9-81$ \\
\hline 31 & 445 & $1-19.66$ & 75 & 633 & $2-24-77$ \\
\hline 31 & 446 & $1.19-66$ & 75 & 634 & $2-24.77$ \\
\hline 31 & 447 & $1-19-66$ & 75 & 635 & $2-24-77$ \\
\hline 31 & 448 & 1.19 .66 & 75 & 636 & $2-24-77$ \\
\hline 31 & 449 & $1-19.66$ & 75 & 637 & $2-24-77$ \\
\hline 31 & 450 & $1-19.66$ & 75 & 638 & $2-24-77$ \\
\hline 41 & 454 & $9.16-80$ & 75 & 639 & $2-24-77$ \\
\hline 48 & 457 & 1.20 .78 & 107 & $640 \mathrm{a}$ & $2-19-82$ \\
\hline 26 & 461 & 8-1-81 & 50 & 641 & $10-15.81$ \\
\hline 26 & 462 & $8-1-81$ & 50 & 642 & $10.15-81$ \\
\hline 26 & 464 & $8-1-81$ & 50 & 643 & $10.15-81$ \\
\hline 26 & 465 & $8-1-81$ & 50 & 644 & $8 \cdot 1.79$ \\
\hline 26 & 466 & $8-1-81$ & 50 & 645 & $8-1-79$ \\
\hline 26 & 467 & $8-1-81$ & 50 & 646 & 8.1 .79 \\
\hline 26 & 468 & 8.1 .81 & 44 & 650 & D \\
\hline 53 & 470 & $10-30-81$ & & & \\
\hline 85 & 474 & 8.27 .81 & & & \\
\hline 85 & 475 & $4-21-81$ & & & \\
\hline 85 & 476 & $\mathrm{D}$ & & & \\
\hline 107 & 477 & $2-23-83$ & & & \\
\hline
\end{tabular}




\begin{tabular}{|c|c|}
\hline Page & SRM \\
\hline 44 & 651 \\
\hline 44 & 652 \\
\hline 50 & $654 a$ \\
\hline 26 & S668 \\
\hline 71 & 670 \\
\hline 43 & 671 \\
\hline 43 & 672 \\
\hline 43 & 673 \\
\hline 107 & 674 \\
\hline 107 & 675 \\
\hline 52 & $680 \mathrm{Ll} A$ \\
\hline 52 & $680 \mathrm{~L} 2 \mathrm{~A}$ \\
\hline 52 & $.681 \mathrm{Ll}$ \\
\hline 52 & $681 \mathrm{~L} 2$ \\
\hline 52 & 682 \\
\hline 52 & 683 \\
\hline 52 & 685 \\
\hline 72 & 688 \\
\hline 35 & 689 \\
\hline 70 & 690 \\
\hline 70 & 691 \\
\hline 70 & 692 \\
\hline 70 & 693 \\
\hline 70 & 696 \\
\hline 71 & 697 \\
\hline 71 & 698 \\
\hline 71 & 699 \\
\hline 114 & $700 d$ \\
\hline 114 & $701 d$ \\
\hline 114 & 703 \\
\hline 90,94 & 705 \\
\hline 90 & 706 \\
\hline 89 & 708 \\
\hline 88,89 & 709 \\
\hline 88 & 710 \\
\hline 88 & 711 \\
\hline 88 & 712 \\
\hline 88 & 713 \\
\hline 88 & 714 \\
\hline 88 & 715 \\
\hline 88 & 716 \\
\hline 88 & 717 \\
\hline 89 & 718 \\
\hline 94 & 720 \\
\hline 55 & $723 a$ \\
\hline 93 & $724 a$ \\
\hline 44 & 726 \\
\hline 45 & 728 \\
\hline 95 & 731 \\
\hline 95 & 733 \\
\hline 95 & $736 a$ \\
\hline 95 & 737 \\
\hline 95 & 738 \\
\hline 95 & 739 \\
\hline 92 & 740 \\
\hline
\end{tabular}

\section{Certificate Date}

D

D

10-15-81

G

D

6-29-79

6-29-79

6-29.79

6-1-83

6-18-82

3-1-77

3-1-77

3.1.77

3-1-77

$2-1.77$

10-1-81

10-1-81

8.18.81

2-24.82

10.24.78

4.12-82

10-24-78

10-24-78

8-24-79

8.24-79

8-24.79

8-12.81

B

1.15.77

11-1-66

11-28-78

2-19-79

9-15.73

6-5-74

6-29-62

7-1-64

10-19-66

10-19-66

10-19-66

9.7 .66

9.7 .66

11-18-69

4-28-72

4-13-82

4-20-81

9.24-73

1.31-67

10-1.81

7.31-72

12-30-71

D

5-19.76

D

5-12-71

2-19-70

\section{Page}

92

93

92

94

94

94

95

95

95

95

92

92

52,109

95

89

88

94

94

25

25

25

25

25

25

25

25

25

25

25

25

34

34

34

34

34

31

31

31

31

39

39
39

39
39

39
39

42

42

42

42

39

39

39

39

39

39

42

36

36

36

43

43

43

56

56

56

56
SRM

741

742

743

745

746

748

763

764

765

766

$767 \mathrm{a}$

768

769

772

773

774

781

782

$803 a$

D803a

$804 a$

$805 a$

$807 a$

D807a

808 a

$809 b$

$817 \mathrm{~b}$

$820 \mathrm{a}$

821

827

837

D837

840

D840

D841

849

D849

850

D850

855

856

858

859

864

865

866

867

871

872

874

875

879

880

882

890

891

892

897

898

899

900

909

910

$911 \mathrm{a}$
Certificate Date

7.18-72

6-5.70

4.22.76

5.14-69

8-11.70

8.10-70

4.5 .73

4.5-73

4.5 .73

4. 5.73

6-9-83

12-13-78

$11-15 \cdot 82$

10-18-78

$11.24-80$

7.19-82

4-1.77

D

5-5-65

5.5 .65

5-5.65

5.5-65

5-5-65

5-5-65

5-5-65

5.5 .65

5.5.65

5.5-65

5-5.65

5.5-65

9.22-59

9.22-59

9.22.59

9-22.59

9.22.59

$1-19.66$

1-19-66

$1-19-66$

$1-19-66$

1.8 .80

1.8 .80

6.6-80

6-6-80

D

D

D

D

8.2-79

8.2-79

1.19-78

$1.19-78$

6-20-79

6-20-79

8.3-79

4. 1.82

4-1-82

4-1-82

8-31-83

8-31-83

8-31-83

4-5.79

7.13-83

5-15-81

3-5.80 


\begin{tabular}{|c|c|c|c|c|c|}
\hline Page & SRM & Certificate Date & Page & SRM & Certificate Date \\
\hline 56 & $912 \mathrm{a}$ & $11-16.79$ & 113 & 1004 & $4-3-72$ \\
\hline 56 & 913 & 11.23 .73 & 115 & $1006 b$ & $4-29-83$ \\
\hline 56 & 914 & $11-23.73$ & 115 & $1007 \mathrm{a}$ & $2-27.76$ \\
\hline 56 & 915 & $11-21.73$ & 115 & 1008 & A \\
\hline 56 & 916 & $3-10-71$ & 115 & $1010 \mathrm{a}$ & $6-21-82$ \\
\hline 56 & 917 & $9-20-73$ & 113 & $1017 \mathrm{a}$ & $9-24-71$ \\
\hline 56 & 918 & $11-23-73$ & 113 & $1018 \mathrm{a}$ & $5-16-73$ \\
\hline 56 & 919 & $11-23-73$ & 113 & $1019 a$ & $\mathrm{D}$ \\
\hline 56 & 920 & $11-23-73$ & 39 & 1034 & $2-3-82$ \\
\hline 56 & 921 & $12-16-73$ & 39 & 1035 & $2-3-82$ \\
\hline 56,83 & 922 & $8-20-76$ & 20 & 1036 & $9-1-82$ \\
\hline 56,83 & 923 & $8-20-76$ & 67 & $1051 b$ & $7-15-68$ \\
\hline 56 & 924 & $11-23.73$ & 67 & $1052 b$ & $3-1-68$ \\
\hline 56 & 925 & $12-26-73$ & 67 & $1053 \mathrm{a}$ & $1-23-70$ \\
\hline 56 & 926 & $7.18-77$ & 67 & $1055 b$ & $7-23-68$ \\
\hline 56 & 927 & 12.31 .79 & 67 & $1057 \mathrm{~b}$ & $7.5-68$ \\
\hline 56 & 928 & 5.19 .76 & 67 & $1059 \mathrm{c}$ & $\mathrm{D}$ \\
\hline 56 & 929 & $4-27-79$ & 67 & $1061 \mathrm{c}$ & $10-1-81$ \\
\hline 56,96 & 930D & A & 67 & $1062 b$ & $4-12.76$ \\
\hline 56,96 & $931 c$ & $2-12-82$ & 67 & $1065 b$ & 11.1 .67 \\
\hline 57,96 & 932 & A & 67 & $1066 \mathrm{a}$ & 4-23-69 \\
\hline 57 & 934 & A & 67 & $1069 b$ & 2-13-69 \\
\hline 57,96 & 935 & $6-1.77$ & 67 & $1070 \mathrm{a}$ & $4-24-64$ \\
\hline 57,96 & 936 & 4.1-79 & 67 & $1071 b$ & $2-26-76$ \\
\hline 57 & 937 & $6-9.78$ & 67 & $1073 b$ & $7-15-67$ \\
\hline 57 & 938 & $5 \cdot 15-81$ & 67 & $1074 \mathrm{a}$ & 5.13 .66 \\
\hline 77 & 945 & 4-6-71 & 67 & $1075 \mathrm{a}$ & $10-25-67$ \\
\hline 77 & 946 & $8 \cdot 19.82$ & 67 & 1076 & $\mathrm{D}$ \\
\hline 77 & 947 & $8-19-82$ & 67 & $1077 \mathrm{a}$ & $2-10-68$ \\
\hline 77 & 948 & $8-19-82$ & 67 & $1078 b$ & $7-25-72$ \\
\hline 55,77 & $949 \mathrm{f}$ & 9.13 .82 & 67 & $1079 b$ & $2-26-69$ \\
\hline 55,78 & $950 \mathrm{~b}$ & 3.1 .78 & 67 & $1080 \mathrm{a}$ & $2-26-69$ \\
\hline 55,80 & 951 & $10-12-71$ & 68 & 1083 & $\mathrm{D}$ \\
\hline 80 & 952 & $10-12-71$ & 68 & 1084 & $8-2-82$ \\
\hline 79 & 953 & $3-12-69$ & 68 & 1085 & $8-2.82$ \\
\hline 55,78 & 960 & 5.12 .72 & 51 & 1086 & $6-16-80$ \\
\hline 80 & 961 & $6-11-74$ & 51 & 1087 & $6-16-80$ \\
\hline 80 & $962 a$ & $\mathrm{D}$ & 51 & 1088 & $6-16-80$ \\
\hline 80 & $963 \mathrm{a}$ & $\mathrm{D}$ & 51 & 1089 & G \\
\hline 80 & 964 & $6-11-74$ & 51 & 1090 & $4-2-69$ \\
\hline 78 & 969 & D & 51 & 1091 & $4-2-69$ \\
\hline 80 & 975 & $3.11-65$ & 51 & 1092 & 4-4-69 \\
\hline 80 & 976 & $3.25-65$ & 51 & 1093 & 3-4-69P \\
\hline 80 & 977 & $3-25-65$ & 51 & 1094 & 6-12-69P \\
\hline 80 & 978 & $3-24-65$ & 46 & 1102 & 11.9 .81 \\
\hline 80 & 979 & $5-3-66$ & 46 & 1103 & $8-13-65$ \\
\hline 80 & 980 & $1-31-67$ & 46 & C1104 & $8-13-65$ \\
\hline 80 & 981 & 4- $10-73$ & 46 & 1106 & $11-23-81$ \\
\hline 80 & 982 & $4-10-73$ & 46 & C1106 & $11-23.81$ \\
\hline 80 & 983 & $4-10-73$ & 46 & 1107 & $11-23-81$ \\
\hline 55,80 & 985 & $8-31.79$ & 46 & $\mathrm{C} 1107$ & $11-23-81$ \\
\hline 55,80 & 987 & 10.1 .82 & 46 & 1108 & $11-23-81$ \\
\hline 80 & 989 & $2 \cdot 19.74$ & 46 & C1108 & $11-23.81$ \\
\hline 80 & 990 & $8-75$ & 46 & 1109 & $12-11-81$ \\
\hline 80 & 991 & 3.19 .76 & 46 & C1109 & $10-1.81$ \\
\hline 78 & 993 & $6-30-75$ & 46 & 1110 & $10-1-81$ \\
\hline 78 & 995 & $8-25-80$ & 46 & C1110 & $10-1-81$ \\
\hline 77 & 996 & $6-10-81$ & 46 & 1111 & $10-1-81$ \\
\hline 57 & 998 & $1-11.83$ & & & \\
\hline 55 & 999 & $9-6.72$ & & & \\
\hline 115 & 1001 & A & & & \\
\hline 115 & $1002 c$ & $12-13-78$ & & & \\
\hline 113 & $1003 a$ & $\mathrm{D}$ & & & \\
\hline
\end{tabular}




$\begin{array}{ll}\text { Page } & \text { SRM } \\ 46 & \text { C1111 } \\ 46 & 1112 \\ 46 & \text { C1112 } \\ 46 & 1113 \\ 46 & \text { C1113 } \\ 46 & 1114 \\ 46 & \text { C1114 } \\ 46 & 1115 \\ 46 & \text { C1115 } \\ 46 & 1116 \\ 46 & \text { C1116 } \\ 46 & 1117 \\ 46 & \text { C1117 } \\ 46 & 1118 \\ 46 & \text { C1118 } \\ 46 & 1119 \\ 46 & \text { C1119 } \\ 46 & 1122 \\ 46 & \text { C1122 } \\ 46 & \text { C1123 } \\ 49 & 1131 \\ 49 & 1132 \\ 26 & 1134 \\ 26 & 1135 \\ 26 & 1136 \\ 38 & 1138 \mathrm{a} \\ 38 & 1139 \mathrm{a} \\ 38 & 1143 \mathrm{a} \\ 38 & 1144 \mathrm{a} \\ 38 & 1145 \\ 38 & 1146 \\ 38 & 1150 \\ 31 & \text { C1151 } \\ 31 & 1151 \mathrm{a} \\ 31 & \mathrm{C} 1152 \\ 31 & 1152 \mathrm{a} \\ 31 & \mathrm{C} 1153 \\ 31 & 1153 \mathrm{a} \\ 31 & \mathrm{C} 1154 \\ 31 & 1154 \mathrm{a} \\ 31 & 1155 \\ 32 & 1156 \\ 34 & 1157 \\ 32 & 1158 \\ 49 & 1159 \\ 49 & 1160 \\ 26 & 1166 \\ 26 & 1169 \mathrm{~b} \\ 31 & 1170 \mathrm{~b} \\ 31 & 1171 \\ 31 & 1172 \\ 38 & 1173 \\ 38 & \mathrm{C} 1173 \\ 33 & 1197 \\ 33 & \text { S1198 } \\ & \\ & \end{array}$

\begin{tabular}{l} 
Certificate Dat \\
$10-1-81$ \\
$10-1-81$ \\
$10-1-81$ \\
$10-1-81$ \\
$10-1-81$ \\
$10-1-81$ \\
$10-1-81$ \\
$11-23-81$ \\
$11-23-81$ \\
$11-23-81$ \\
$11-23-81$ \\
$11-23-81$ \\
$11-23-81$ \\
$12-11-81$ \\
$7-12-82$ \\
$12-11-81$ \\
$7-12-82$ \\
$12-11-81$ \\
$12-11-81$ \\
$12-11-81$ \\
$10-1-81$ \\
$1-30-70$ \\
$4-30-70$ \\
$7-27-72$ \\
$8-31-73$ \\
$1-20-77$ \\
$1-20-77$ \\
$12-1-76$ \\
$12-1-76$ \\
$5-16-78$ \\
$5-16-78$ \\
$5-16-78$ \\
$1-31-80$ \\
$D$ \\
$1-31-80$ \\
$D$ \\
$1-31-80$ \\
$D$ \\
$1-31-80$ \\
$D$ \\
$8-4-69$ \\
$8-26-81$ \\
$8-6-73$ \\
$12-30-77$ \\
$8-4-81$ \\
$8-4-81$ \\
$8-1-81$ \\
$4-15-82$ \\
$1-30-74$ \\
$7-7-71$ \\
$7-7-71$ \\
$5-16-83$ \\
\\
\hline-84 \\
\hline
\end{tabular}

Page

33

33

33

33

33

33

26

26

26

26

26

26

26

51

51

51

51

51

51

33

33

33

33

48

48
48

48

26

45

45

26

26

26

26

26

31

26

26

46

46

26

26

31

31

31
86

86
86

86
85

85
85

86

86

86

86

87

87

87
87

87
87$$
\begin{aligned}
& 87 \\
& 94
\end{aligned}
$$$$
94
$$

94,108

94,108

94,108

94,108

94,108
SRM

S1199

1206-2

1207-1

1207.2

$1208-1$

1208-2

C1221

1222

1224

1225

1226

1227

1228

1234

1235

1236

1237

1238

1239

1244

1245

1246

1247

C1251

C1252

C1253

1254

1258

1259

$1261 \mathrm{a}$

$1262 \mathrm{a}$

$1263 \mathrm{a}$

$1264 \mathrm{a}$

$1265 \mathrm{a}$

1267

1269

1270

1275

1276

C 1285

1286

C1287

C1288

C1289

1359

1360

$1361 \mathrm{~b}$

$1362 \mathrm{a}$

$1363 a$

$1364 \mathrm{a}$

$1365 \mathrm{a}$

$1366 \mathrm{a}$

$1367 \mathrm{a}$

1379

1380

$1398 \mathrm{a}$

$1399 \mathrm{~b}$

$1450 \mathrm{~b}$

1451

1460

1461

1462

1463

1464
Certificate Date

H

8-1.81

8-1-81

8-1-81

8-1-81

8-1-81

1.20-82

10-3-78

2-24-81

3-16-83

12.14-82

3-30-83

9.28-82

11-28-80

$11-28-80$

11-28-80

11-28-80

$11.28-80$

11.28.80

D

D

D

D

9-16-80

9-16-80

9. $16-80$

4-1-82

5-3.78

5.3.78

2-24-81

2-24-81

2-24.81

2-24-81

2-24-81

1.19 .76

6-11-81

6-11-81

3-10-80

3-10-80

6.8-82

6.8-82

6-11-81

8.1-81

6-11.81

A

A

A

A

A

A

A

A

A

A

A

A

5.21-82

D

1.17.79

$1-17.79$

$1-17.79$

$1-17.79$

1.17 .79 


\begin{tabular}{|c|c|c|c|c|c|}
\hline Page & SRM & Certificate Date & Page & SRM & Certificate Date \\
\hline 94,108 & 1465 & $1-17.79$ & 93 & 1651 & $11-12-68$ \\
\hline 94,108 & 1466 & $1-17-79$ & 93 & 1652 & 11.12 .68 \\
\hline 94,108 & 1467 & 1.17 .79 & 93 & 1653 & $11-12-68$ \\
\hline 94,108 & 1468 & $1-17-79$ & 93 & 1654 & $4-7-71$ \\
\hline 108 & 1470 & $2-23-82$ & 93 & 1655 & $3-20-81$ \\
\hline 90,94 & 1475 & $12 \cdot 8 \cdot 78$ & 59 & $1658 \mathrm{a}$ & A \\
\hline 90 & 1476 & $11-6-69$ & 59 & $1659 a$ & A \\
\hline 90 & 1478 & 1.17 .79 & 59 & $1660 \mathrm{a}$ & A \\
\hline 90 & 1479 & $3-27-81$ & 59 & $1661 \mathrm{a}$ & A \\
\hline 90 & 1482 & $10.18-76$ & 59 & $1662 a$ & A \\
\hline 90 & 1483 & $3-9-76$ & 59 & $1663 a$ & A \\
\hline 90 & 1484 & $10-18-76$ & 59 & $1664 \mathrm{a}$ & A \\
\hline 91 & 1490 & $2-21-77$ & 59 & $1665 b$ & A \\
\hline 111 & 1495 & 3.13 .81 & 59 & $1666 b$ & A \\
\hline 108 & 1521 & A & 59 & $1667 b$ & A \\
\hline 108 & 1522 & A & 59 & $1668 b$ & A \\
\hline 108 & 1523 & A & 59 & $1669 b$ & A \\
\hline 106 & 1541 & $10-1-81$ & 59 & 1670 & $12-9-82$ \\
\hline 57 & 1549 & D & 59 & 1671 & 12.9 .82 \\
\hline 57 & 1566 & 12.12 .79 & 59 & 1672 & $12-9-82$ \\
\hline 57 & 1567 & $1-3-78$ & 59 & $1674 \mathrm{~b}$ & A \\
\hline 57 & 1568 & $1-3-78$ & 59 & $1675 b$ & A \\
\hline 57 & 1569 & $9-7-76$ & 59 & $1677 \mathrm{c}$ & A \\
\hline 57 & 1572 & $12.20-82$ & 59 & $1678 \mathrm{c}$ & A \\
\hline 57 & 1573 & $10-18-76$ & 59 & $1679 \mathrm{c}$ & A \\
\hline 57 & 1575 & 10.18 .76 & 59 & $1680 b$ & A \\
\hline 57 & $1577 \mathrm{a}$ & $6-15-82$ & 59 & $1781 \mathrm{~b}$ & A \\
\hline 61 & 1579 & 1.23 .73 & 59 & $1683 b$ & A \\
\hline 64 & 1580 & $11-24-80$ & 59 & $1684 b$ & A \\
\hline 65 & 1581 & $6.25-82$ & 59 & $1685 b$ & A \\
\hline 64 & 1582 & $\mathrm{D}$ & 59 & $1686 b$ & A \\
\hline 57 & 1590 & $12.11-80$ & 59 & $1687 b$ & A \\
\hline 57 & 1595 & $7.6-83$ & 59,113 & 1690 & $12-22-82$ \\
\hline 57 & 1599 & $8-2-82$ & 59,113 & 1691 & $\mathrm{D}$ \\
\hline 112 & 1600 & A & 59 & 1693 & $3-20-82$ \\
\hline 61 & 1618 & $\mathrm{D}$ & 59 & 1694 & $3-20-82$ \\
\hline 61 & 1619 & $12-22-81$ & 59 & 1696 & $3-20-82$ \\
\hline 61 & $1620 \mathrm{a}$ & $12-22.81$ & 59 & 1805 & $12.27-82$ \\
\hline 61 & $1621 a$ & $12 \cdot 22 \cdot 81$ & 59 & 1806 & 12.27 .82 \\
\hline 61 & $1622 b$ & $12-22-81$ & 59 & 1808 & $6-13.83$ \\
\hline 61 & $1623 a$ & $12-22-81$ & 116 & 1810 & 5.5 .72 \\
\hline 61 & $1624 \mathrm{a}$ & 12.22 .81 & 108 & $1815 \mathrm{a}$ & $\mathrm{D}$ \\
\hline 61 & 1625 & A & 108 & $1816 a$ & D \\
\hline 61 & 1626 & A & 106 & 1817 & $5-10-83$ \\
\hline 61 & 1627 & A & 99 & 1820 & $9-30-74$ \\
\hline 61 & $1629 a$ & A & 99 & 1822 & $\mathrm{D}$ \\
\hline 61 & 1630 & $8-1.79$ & 99 & 1823 & $12-9-76$ \\
\hline 62 & $1632 \mathrm{a}$ & $10-24-83$ & 90 & 1825 & $\mathrm{D}$ \\
\hline 62 & $1633 a$ & $4-18-79$ & 90 & 1826 & D \\
\hline 62 & $1634 a$ & $2-19-82$ & 90 & 1827 & D \\
\hline 62 & 1635 & $8-22-79$ & 74 & 1830 & $7-18-82$ \\
\hline 62 & $1636 a$ & $2-5-80$ & 74 & 1831 & $7-18-82$ \\
\hline 62 & $1637 a$ & $2-5-80$ & 90 & 1840 & $5.14-82$ \\
\hline 62 & $1638 \mathrm{a}$ & $2-5.80$ & 90 & 1841 & $5-14-82$ \\
\hline 64 & 1639 & $4-20-83$ & 109 & 1850 & $12-10-80$ \\
\hline 62 & $1641 \mathrm{~b}$ & $4-13-83$ & 107 & 1857 & 3.8 .83 \\
\hline 62 & $1642 b$ & $7-15.82$ & 109 & 1860 & $8 \cdot 2-82$ \\
\hline 62 & $1643 a$ & $7 \cdot 19.82$ & & & \\
\hline 64 & 1644 & $4 \cdot 27.81$ & & & \\
\hline 62 & 1645 & 5.5 .82 & & & \\
\hline 62 & 1646 & 6.7 .82 & & & \\
\hline 64 & 1647 & $12 \cdot 7.81$ & & & \\
\hline 62 & 1648 & $5.11-82$ & & & \\
\hline 64 & 1649 & $4-8.82$ & & & \\
\hline
\end{tabular}




\begin{tabular}{|c|c|c|c|c|c|}
\hline Page & SRM & Certificate Date & Page & SRM & Certificate Date \\
\hline 109 & 1861 & $8-2-82$ & 96 & 2601 & A \\
\hline 109 & 1862 & $8-2-82$ & 60 & $2612 a$ & A \\
\hline 109 & 1863 & $8-2-82$ & 60 & $2613 a$ & A \\
\hline 53 & 1871 & $\mathrm{D}$ & 60 & $2614 a$ & A \\
\hline 53 & 1872 & $\mathrm{D}$ & 60 & $2619 a$ & A \\
\hline 53 & 1873 & $\mathrm{D}$ & 60 & $2620 a$ & A \\
\hline 53 & 1874 & $\mathrm{D}$ & 60 & $2621 \mathrm{a}$ & A \\
\hline 53 & 1875 & $\mathrm{D}$ & 60 & $2622 a$ & A \\
\hline 67. & 1876 & $6-9.83$ & 60 & $2623 a$ & A \\
\hline 66 & 1878 & $11-3-83$ & 60 & $2624 a$ & A \\
\hline 75 & 1880 & $\mathrm{D}$ & 60 & $2625 a$ & A \\
\hline 75 & 1881 & $\mathrm{D}$ & 60 & $2626 a$ & A \\
\hline 107 & 1890 & $5-16-83$ & 60 & 2627 & A \\
\hline 89 & 1894 & $\mathrm{D}$ & 60 & 2628 & A \\
\hline 89 & 1895 & $\mathrm{D}$ & 60 & 2629 & A \\
\hline 112 & 1901 & $3-1.76$ & 60 & 2630 & A \\
\hline 61 & 1911 & 8.4 .82 & 60 & 2631 & A \\
\hline 95 & 1967 & 2.23 .77 & 60 & 2632 & A \\
\hline 57,93 & 1968 & $6-77$ & 60 & 2633 & A \\
\hline 57,93 & 1969 & D & 60 & 2634 & A \\
\hline 57 & 1970 & D & 60 & 2635 & A \\
\hline 98 & $2003 a$ & $3-30-81$ & 60 & 2636 & A \\
\hline 98 & $2008 \mathrm{a}$ & $\mathrm{D}$ & 60 & 2637 & A \\
\hline 96 & 2009 & D & 60 & 2638 & A \\
\hline 96 & 2010 & $\mathrm{D}$ & 60 & 2639 & A \\
\hline 98 & 2015 & $5-5-82$ & 60 & 2640 & A \\
\hline 98 & 2016 & $5-5-82$ & 60 & 2641 & A \\
\hline 98 & $2019 b$ & $10-13-83$ & 60 & 2642 & A \\
\hline 98 & 2020 & $3.30-82$ & 60 & 2643 & A \\
\hline 98 & 2021 & $9-16-80$ & 60 & 2644 & A \\
\hline 98 & 2022 & $9-16-80$ & 60 & 2645 & A \\
\hline 98 & 2023 & $4-16-81$ & 60 & 2646 & A \\
\hline 98 & 2024 & 4-16-81 & 60 & 2647 & A \\
\hline 98 & 2025 & $2-23-82$ & 60 & 2648 & A \\
\hline 96 & 2030 & A & 60 & 2649 & A \\
\hline 96 & 2031 & A & 60 & 2650 & A \\
\hline 96 & 2032 & 10.31 .79 & 60 & 2651 & A \\
\hline 96 & 2033 & D & 60 & 2652 & A \\
\hline 115 & 2061 & $12-11-80$ & 60 & 2653 & A \\
\hline 84 & 2069 & $11-29-83$ & 60 & 2654 & A \\
\hline 114 & 2106 & C & 60 & 2655 & A \\
\hline 114 & 2107 & $\mathrm{C}$ & 60 & 2656 & A \\
\hline 56 & 2141 & $8-28-70$ & 60 & 2657 & A \\
\hline 56 & 2142 & $9-1.70$ & 60 & 2658 & A \\
\hline 56 & 2143 & $1-12.82$ & 60 & 2659 & A \\
\hline 56 & 2144 & $4-5.73$ & 66 & 2670 & D \\
\hline 93 & 2152 & D & 66 & $2671 \mathrm{a}$ & $12-29-82$ \\
\hline 84 & $2186 \mathrm{I}$ & $5-28-68$ & 66 & $2672 a$ & $5-16-83$ \\
\hline 84 & 218611 & $5-28-68$ & 66 & 2673 & $6-25-79$ \\
\hline 84 & 2191 & $5-28-68$ & 66 & 2674 & $12-18-79$ \\
\hline 84 & 2192 & $5-28.68$ & 66 & 2675 & $8-19-75$ \\
\hline 84 & 2201 & $2-22-71$ & 66 & $2676 b$ & 1.11 .83 \\
\hline 84 & 2202 & $2-22 \cdot 71$ & 66 & $2679 a$ & D \\
\hline 84 & 2203 & 5.21 .73 & 65 & 2682 & 2.7 .83 \\
\hline 108 & 2526 & $8-1-83$ & 65 & 2683 & $2-7.83$ \\
\hline 108 & 2527 & $8-1-83$ & 65 & 2684 & $2-7.83$ \\
\hline 108 & 2528 & $\mathrm{D}$ & 65 & 2685 & $2-7.83$ \\
\hline \multirow[t]{7}{*}{108} & 2529 & $\mathrm{D}$ & 112 & 3200 & A \\
\hline & & & 112 & 3210 & A \\
\hline & & & 112 & 3216 & A \\
\hline & & & 103 & $4200 B$ & A \\
\hline & & & 103 & $4201 \mathrm{~B}$ & A \\
\hline & & & 103 & $4206 C$ & A \\
\hline & & & 103 & 4207 & A \\
\hline
\end{tabular}




\begin{tabular}{|c|c|c|c|c|c|}
\hline Page & SRM & Certificate Date & Page & SRM & Certificate Date \\
\hline 103 & 4213 & A & 104 & $4952 B$ & $\mathrm{D}$ \\
\hline 103 & $4218 \mathrm{E}$ & A & 104 & $4953 \mathrm{C}$ & $5-78$ \\
\hline 101 & 4226 & 8.69 & 104 & 4956 & $3-1-68$ \\
\hline 101 & 4229 & $5-72$ & 104 & 4957 & $3-1.68$ \\
\hline 101 & $4233 \mathrm{~B}$ & $11-79$ & 104 & 4958 & $3-1-68$ \\
\hline 103 & 4235 & A & 104 & 4959 & $3-1.68$ \\
\hline 103 & $4241 B$ & A & 104 & 4960 & $3-1-68$ \\
\hline 101 & 4245 & $5-74$ & 104 & 4961 & $3-1-68$ \\
\hline 101 & 4246 & 5.74 & 104 & 4962 & 3.1 .68 \\
\hline 101 & $4250 B$ & 4.82 & 104 & 4963 & $3 \cdot 1.69$ \\
\hline 101 & $4251 \mathrm{~B}$ & $12-81$ & 104 & $4964 B$ & $12-31-65$ \\
\hline 101 & 4257 & 4-79 & 102 & $4990 \mathrm{C}$ & $\mathrm{D}$ \\
\hline 104 & $4260 \mathrm{C}$ & A & 103 & $4997 \mathrm{E}$ & 6.79 \\
\hline 104 & $4264 B$ & $9-82$ & 112 & 6250 & A \\
\hline 104 & 4266 & 2.81 & 119 & 8000 & 3.24 .83 \\
\hline 103 & $4275 B$ & $7-83$ & 119 & 8001 & $2-6-74$ \\
\hline 101 & $4276 B$ & $7-83$ & 119 & 8002 & $2 \cdot 6-74$ \\
\hline 101 & 4288 & 11.82 & 119 & 8003 & $2-6-74$ \\
\hline 103 & 4307 I & $\mathrm{D}$ & 119 & 8004 & $2-6.74$ \\
\hline 103 & $4308 \mathrm{C}$ & $\mathrm{A}$ & 119 & 8005 & 1.78 \\
\hline 103 & $4309 \mathrm{G}$ & $\mathrm{D}$ & 119 & 8006 & $1-78$ \\
\hline 103 & $4310 B$ & A & 119 & 8007 & 1.78 \\
\hline 101 & 4331 & 3.75 & 119 & 8008 & $3-80$ \\
\hline 101 & $4334 \mathrm{~B}$ & 7.79 & 62,79 & 8505 & D \\
\hline 101 & 4338 & $8-80$ & 106 & 9910 & $\mathrm{C}$ \\
\hline 105 & $4350 \mathrm{~B}$ & $9-9-81$ & 81 & 9940 & $\mathrm{C}$ \\
\hline 105 & 4351 & $\mathrm{D}$ & 81 & 9941 & $\mathrm{C}$ \\
\hline 105 & 4352 & $\mathrm{D}$ & 81 & 9942 & $\mathrm{C}$ \\
\hline 105 & 4353 & $4-81$ & 81 & 9943 & C \\
\hline 105 & 4355 & $\mathrm{D}$ & 78 & U-0002 & $4-6-81$ \\
\hline 101,102 & 4361 & 1.81 & 78 & U-005a & D \\
\hline 101 & $4370 \mathrm{~B}$ & $5-78$ & 78 & U-010 & $4-6-81$ \\
\hline 105 & $4400 \mathrm{~L} \cdot \mathrm{F}$ & $7-83$ & 78 & U.015 & $4-6-81$ \\
\hline 105 & 4401 L-I & 1.83 & 78 & U-020a & $\mathrm{D}$ \\
\hline 106 & 4402L-C & $10-80$ & 78 & U-030a & $\mathrm{D}$ \\
\hline 106 & 4403L-B & $4-77$ & 78 & U-050 & $4-6-81$ \\
\hline 106 & 4404L-F & $6-83$ & 78 & U. 100 & $4-6-81$ \\
\hline 105 & $4405 \mathrm{~L}-\mathrm{B}$ & 9.78 & 78 & U. 150 & $4-6-81$ \\
\hline 105 & 4406L-G & $\mathrm{D}$ & 78 & U. 200 & $4-6-81$ \\
\hline 105 & 4407L-H & 12.82 & 78 & U.350 & $4-6-81$ \\
\hline 105 & 4408L-C & 1.81 & 78 & U. 500 & $4-6 \cdot 81$ \\
\hline 105 & 4409L-D & 9.81 & 78 & U.750 & $4-6.81$ \\
\hline 106 & $4410 \mathrm{H}-\mathrm{I}$ & D & 78 & U.800 & $4 \cdot 6-81$ \\
\hline 105 & $4411 \mathrm{~L} \cdot \mathrm{B}$ & $2-79$ & 78 & U. 850 & $4-6.81$ \\
\hline 105 & 4412L-H & 3.83 & 78 & U.900 & $4-6-81$ \\
\hline 105 & 4414L-C & $6-80$ & 78 & U.930 & $4 \cdot 6-81$ \\
\hline 103,106 & 4415L-I & D & 78 & U-970 & $4-6-81$ \\
\hline 105 & $4416 \mathrm{~L} \cdot \mathrm{D}$ & S. 83 & 116 & RM 1C & $\mathrm{E}$ \\
\hline 105 & $4417 \mathrm{~L} \cdot \mathrm{C}$ & $\mathrm{D}$ & 116 & RM 1R & $\mathrm{E}$ \\
\hline 105 & $4418 \mathrm{~L}$ & $11-76$ & 116 & RM 5 & $\mathrm{E}$ \\
\hline 106 & 4419L-B & 9.80 & 117 & RM 31 & $\mathrm{E}$ \\
\hline 105 & $4420 \mathrm{~L}$ & D & 105,117 & RM 45B & $\mathrm{E}$ \\
\hline 105 & $4421 \mathrm{~L}$ & $1-80$ & 117 & RM 50 & $\mathrm{E}$ \\
\hline 101 & $4422 \mathrm{~L}$ & 4.80 & 118 & GM 754 & $\mathrm{~F}$ \\
\hline 102 & $4904 \mathrm{~F}$ & $\mathrm{D}$ & 118 & GM 757 & $\mathrm{~F}$ \\
\hline 102 & $4906 \mathrm{~B}$ & 7.78 & 118 & GM 758 & $\mathrm{~F}$ \\
\hline 102 & 4907 & $11-79$ & 118 & GM 759 & $\mathrm{~F}$ \\
\hline 101 & $4915 \mathrm{D}$ & $\mathrm{D}$ & 118 & GM 760 & $\mathrm{~F}$ \\
\hline 101 & $4926 \mathrm{C}$ & 1.79 & 118 & GM 761 & $\mathrm{~F}$ \\
\hline 103 & $4935 C$ & $7-23-74$ & & & \\
\hline 101 & 4943 & $\mathrm{D}$ & & & \\
\hline 101 & 4945D & $11-82$ & & & \\
\hline 101 & 4947 & $4-79$ & & & \\
\hline 101 & 4949B & $2-82$ & & & \\
\hline
\end{tabular}




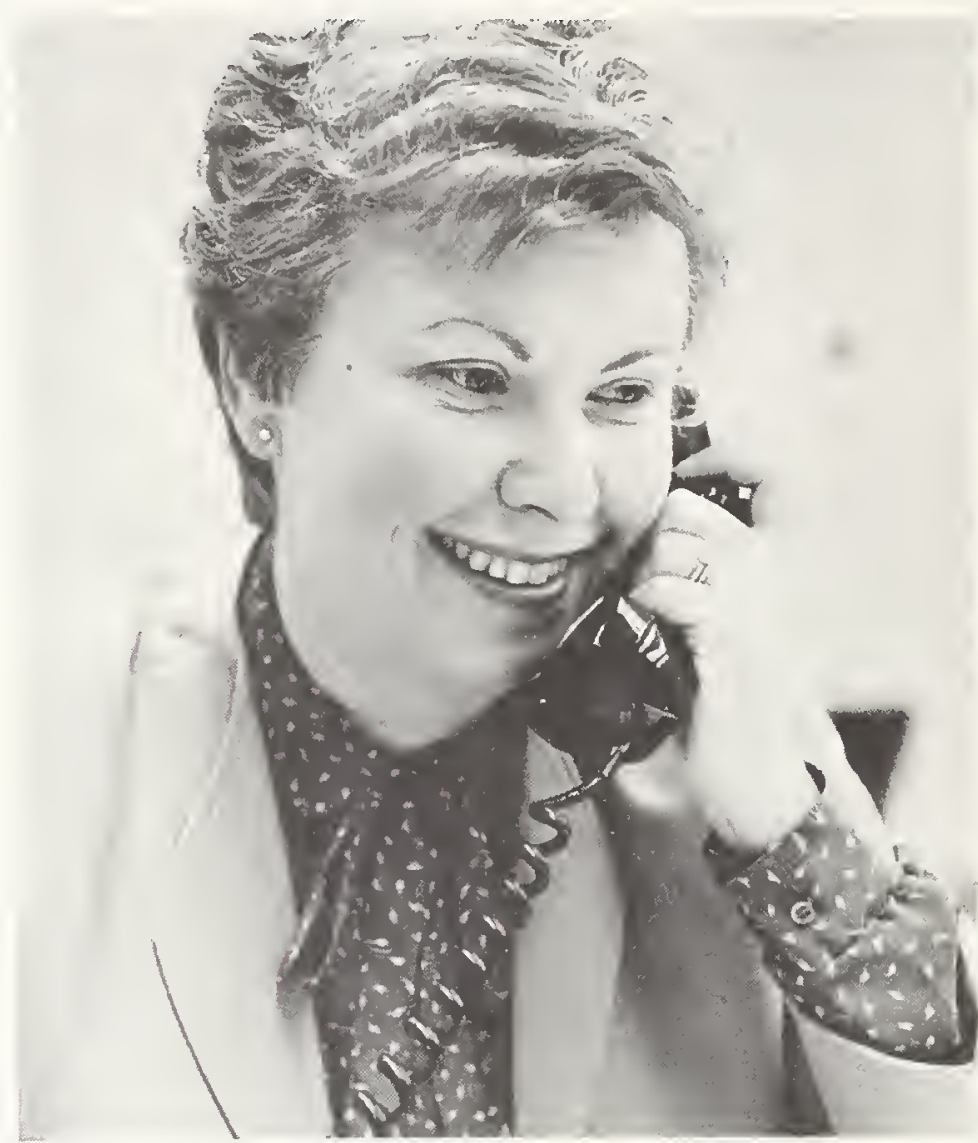

Marketing and advertising

assignments are handled by

Catherine Hudson, editor of the

current SRM catalog. 



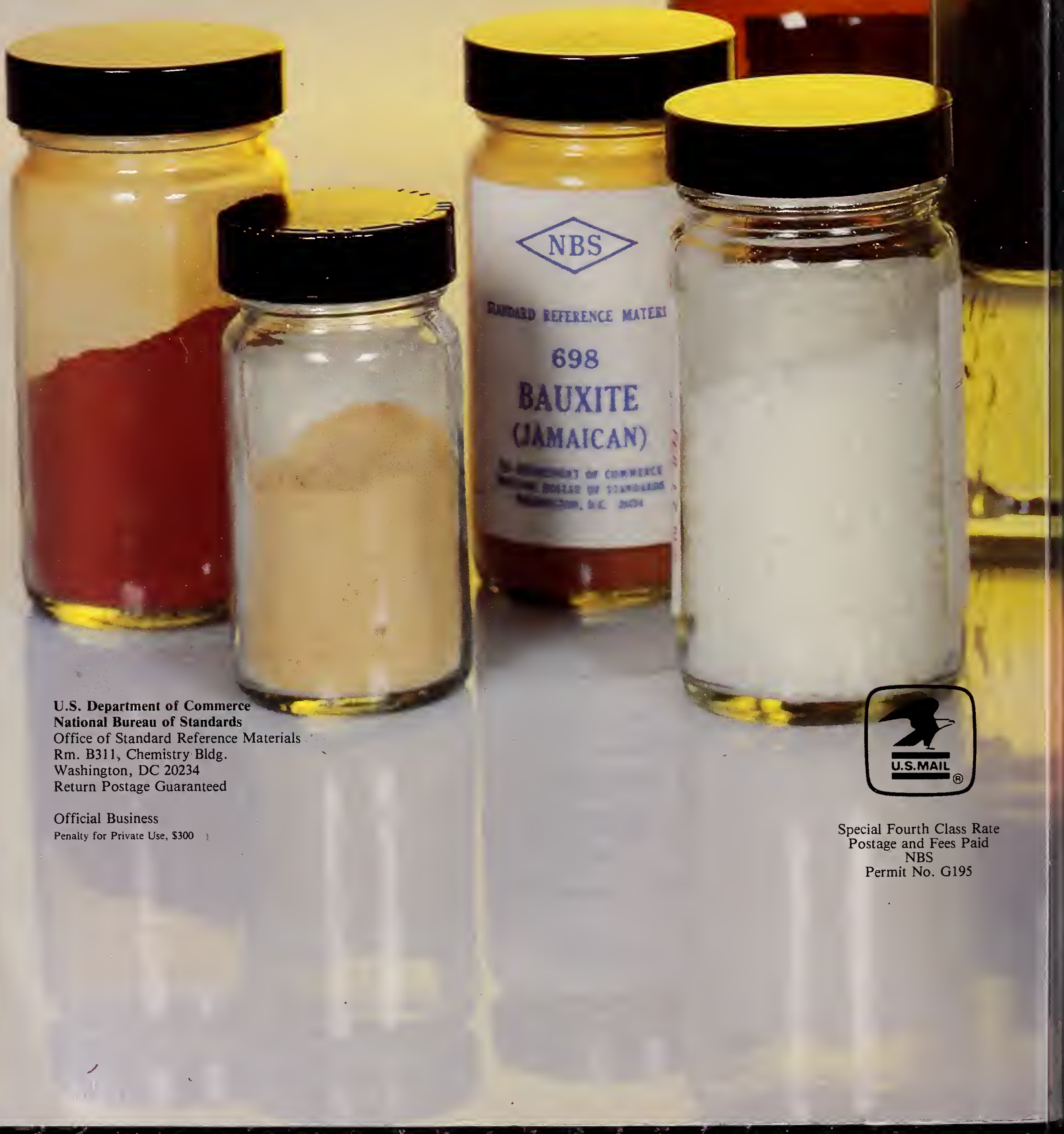

